Supporting Information for

\title{
Enantioselective Silicon-Directed Nazarov Cyclization
}

Jin $\mathrm{Cao}^{\ddagger}$, Meng-Yang $\mathrm{Hu}^{\ddagger}$, Si-Yuan Liu, Xin-Yu Zhang, Shou-Fei Zhu*, and Qi-Lin

Zhou

The State Key Laboratory and Institute of Elemento-Organic Chemistry, College of Chemistry, Nankai University, Tianjin 300071, China

${ }^{\ddagger}$ These authors contribute equally to this work.

Email:sfzhu@nankai.edu.cn

Contents:

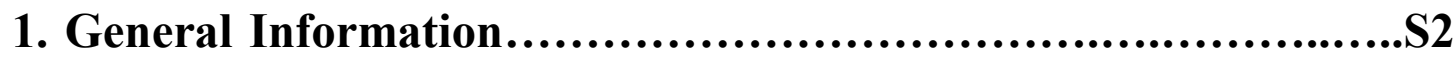

2. Synthesis and Analytical Data of $\beta$-Silyl Divinyl Ketones...........S3

3. Procedures of Enantioselective Silicon-Directed Nazarov

Cyclization............................................................S31

4. Analytical Data of Cyclization Products...........................S34

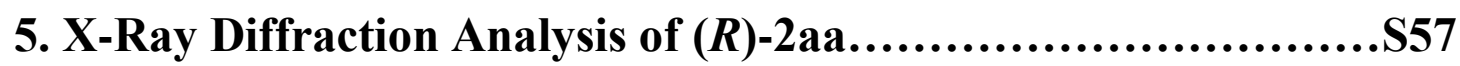

6. Transformations of Cyclization Product 2aa......................S58

7. Procedure of React IR Experiments.............................S62

8. NMR Spectra of New Compounds...............................S66

9. HPLC Charts of Chiral Products.............................S148

10. References................................................S189 


\section{General Information}

Unless otherwise noted, all solvents used in the reactions were distilled from appropriate drying agents prior to use. ${ }^{1} \mathrm{Zn}(\mathrm{OTf})_{2}$ (98\%) was purchased from Sigma-Aldrich Chemical Company and used without further purification. All reactions and manipulations which are sensitive to moisture or air were performed in an argon-filled glovebox (MBRAUN LABstar) or using standard Schlenk techniques. ${ }^{1} \mathrm{H}$ NMR and ${ }^{13} \mathrm{C}$ NMR spectra were recorded with a Brucker AV 400 spectrometer at $400 \mathrm{MHz}\left({ }^{1} \mathrm{H} \mathrm{NMR}\right)$ and $101 \mathrm{MHz}\left({ }^{13} \mathrm{C} \mathrm{NMR}\right)$ in $\mathrm{CDCl}_{3}$. Chemical shifts were reported in ppm down field from internal $\mathrm{Me}_{4} \mathrm{Si}$. HRMS were recorded on an IonSpec FT-ICR mass spectrometer and Waters GCT Premier mass spectrometer. HPLC analysis was performed on an Agilent 1260 Infinity LC chromatography. Melting points were measured on a RY-I or SGW X-4 apparatus and uncorrected. Optical rotations were determined by a Rudolph Autopol VI polarimeter. 


\section{Synthesis and Analytical Data of $\beta$-Silyl Divinyl Ketones}

\subsection{Synthesis of divinyl ketones}

Divinyl ketones were synthesized following the procedures described below. Substrates 1aa-1af, 1ah, 1aj-1al, 1ba-1bd, 1bg-1bi, 1ga-1gc, 1ia were prepared according to Route A. Substrates 1ca-1cc, $\mathbf{1 b e}, \mathbf{1 b f}$ were prepared according to Route B. Substrates 1ag, 1ai, 1ea were prepared according to Route C. Substrates 1db-1de were prepared according to Route D. Substrates 1da were prepared according to Route E. Substrates 1fa were prepared according to Route F. Substrates 1ha were prepared according to Route G. Substrates 1la were prepared according to Route H.

\section{Route $\mathbf{A}^{2,3}$}

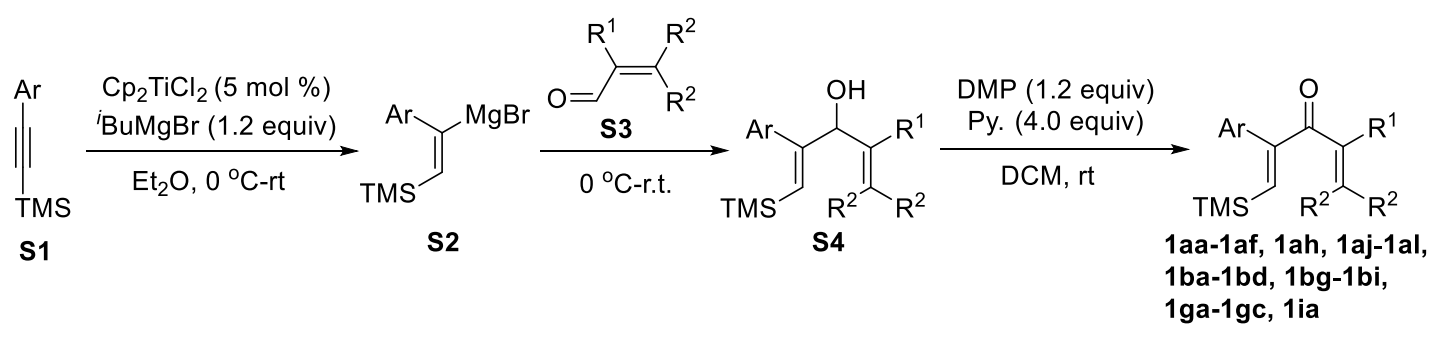

To a solution of isobutylmagnesium bromide $(4.5 \mathrm{mmol})$ in $\mathrm{Et}_{2} \mathrm{O}(7 \mathrm{~mL})$ was added $\mathrm{Cp}_{2} \mathrm{TiCl}_{2}(50 \mathrm{mg}, 0.2 \mathrm{mmol})$ at $0{ }^{\circ} \mathrm{C}$ under $\mathrm{Ar}$, and the mixture was stirred at that temperature for $30 \mathrm{~min}$. To this solution was added 1-aryl-2-silylacetylene S1 (4.0 mmol), and the mixture was stirred at $25{ }^{\circ} \mathrm{C}$ for $6 \mathrm{~h}$. After being cooled to $0{ }^{\circ} \mathrm{C}$, aldehyde $\mathbf{S 3}$ (3.5 mmol), which were prepared according to reported procedures, ${ }^{4-6}$ was added and the mixture was stirred at $25{ }^{\circ} \mathrm{C}$ for $2 \mathrm{~h}$, quenched with saturated aqueous $\mathrm{NH}_{4} \mathrm{Cl}$ solution $(25 \mathrm{~mL})$, and extracted with $\mathrm{Et}_{2} \mathrm{O}(2 \times 30 \mathrm{~mL})$. The combined organic layers were washed with saturated aqueous $\mathrm{NH}_{4} \mathrm{Cl}$ solution $(20 \mathrm{~mL})$ and water $(3 \times 20 \mathrm{~mL})$ and dried over $\mathrm{MgSO}_{4}$. The solvent was evaporated under reduced pressure and the residue was purified by column chromatography on silica gel to afford the desired dienol S4.

To a solution of the Dess-Martin periodinane (DMP, $5.1 \mathrm{~g}, 12.0 \mathrm{mmol}$ ) in DCM (50 mL), pyridine $(3.2 \mathrm{~g}, 40.0 \mathrm{mmol})$ was added. The resulting mixture was stirred for $5 \mathrm{~min}$, then a solution of dienol $\mathbf{S 4}(10.0 \mathrm{mmol})$ in DCM $(40 \mathrm{~mL})$ was added dropwise. 
Stirring was continued for another $30 \mathrm{~min}$ and the reaction mixture was diluted and washed with the mixture of saturated aqueous $\mathrm{NaHCO}_{3}$ and saturated aqueous $\mathrm{Na}_{2} \mathrm{~S}_{2} \mathrm{O}_{3}(1: 1, \mathrm{v} / \mathrm{v})$ for 3 times, then dried over anhydrous $\mathrm{Na}_{2} \mathrm{SO}_{4}$. The solvent was evaporated under reduced pressure and the residue was purified by column chromatography on silica gel to afford the desired dienone $\mathbf{1}$.

\section{Route $\mathbf{B}^{7}$}

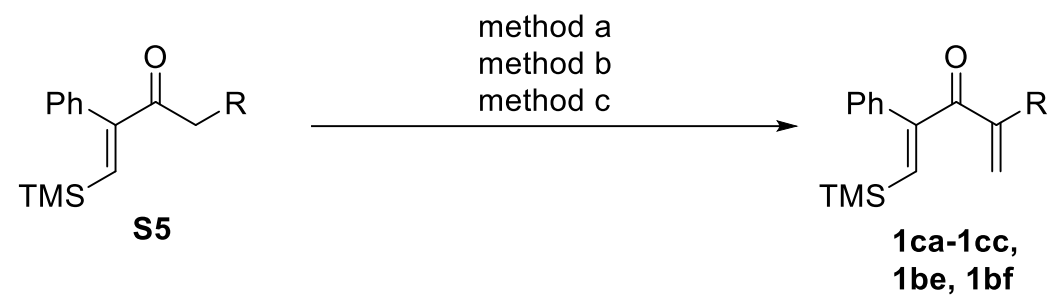

Method a: To a mixture of aqueous formaldehyde solution ( $37 \%$ formaldehyde in water, $200 \mathrm{mg}, 2.5 \mathrm{mmol}$ ) and ketone $\mathbf{S 5}$ (660 mg, $2.2 \mathrm{mmol})$, which was prepared according to Route $\mathrm{A}$, in ${ }^{i} \mathrm{PrOH}(1 \mathrm{~mL})$ were added propionic acid $(16.3 \mathrm{mg}, 0.2$ mmol) and pyrrolidine (15.6 mg, $0.2 \mathrm{mmol})$. The reaction mixture was stirred at $45{ }^{\circ} \mathrm{C}$ for $8 \mathrm{~h} . \mathrm{NaHCO}_{3}$ was added, and the mixture was extracted with $\mathrm{DCM}(3 \times 5 \mathrm{~mL})$. The combined extracts were washed with brine, dried over $\mathrm{Na}_{2} \mathrm{SO}_{4}$. The solvent was evaporated under reduced pressure and the residue was purified by column chromatography on silica gel to afford the desired dienone 1ca.

Method b: To a solution of ketone S5 (560 mg, $1.9 \mathrm{mmol})$ and $\mathrm{Et}_{3} \mathrm{~N}(1.9 \mathrm{~g}, 18.7$ mmol) in DCM (50 mL), was added portion wise $\mathrm{Me}_{2} \mathrm{~N}^{+}=\mathrm{CH}_{2} \mathrm{I}^{-}(690 \mathrm{mg}, 3.7 \mathrm{mmol})$. The mixture was stirred at r.t. until the starting material had been consumed $\left({ }^{1} \mathrm{H}\right.$ NMR monitoring). The mixture was then washed with saturated $\mathrm{NaHCO}_{3}$ and brine, dried over $\mathrm{MgSO}_{4}$, and concentrated under reduced pressure and the residue was purified by column chromatography on silica gel to afford the desired dienone 1cc.

Method c: Bis(dimethylamino)methane $(20.0 \mathrm{mmol})$ and acetic anhydride (20.0 mmol) was added successively to a solution of ketone $\mathbf{S 5}(2.0 \mathrm{mmol})$ dissolved in anhydrous DMF $(2 \mathrm{~mL})$. The mixture was stirred at $95{ }^{\circ} \mathrm{C}$ until the consumption of starting material, monitored by ${ }^{1} \mathrm{H}$ NMR. After cooled to room temperature, the brown mixture was distilled under reduced pressure and the residue was purified by 
column chromatography on silica gel to afford the desired dienone $\mathbf{1}$.

\section{Route $\mathbf{C}^{8,9}$}
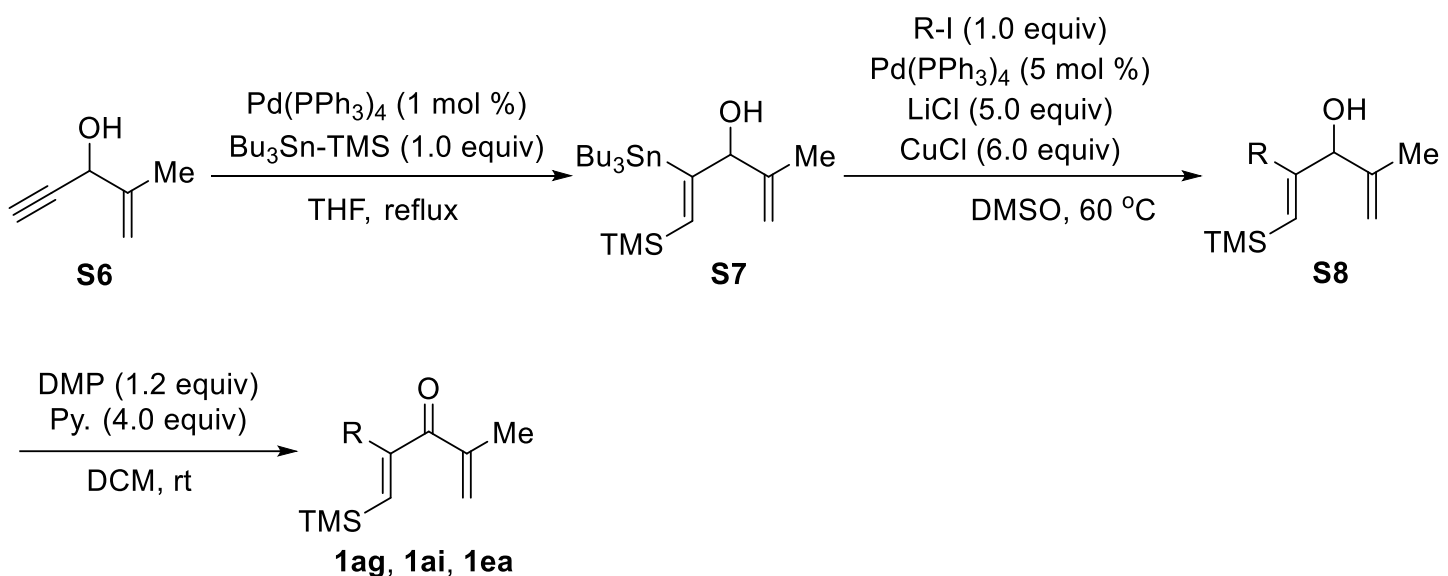

Equimolar amounts of the alkyne $\mathbf{S 6}$ and stannylsilane $(60 \mathrm{mmol})$ were mixed in THF (50 mL) and $\mathrm{Pd}\left(\mathrm{PPh}_{3}\right)_{4}(693 \mathrm{mg}, 0.6 \mathrm{mmol})$ was added under Ar, The resulting mixture was stirred at reflux for $48 \mathrm{~h}$. The mixture was then concentrated under reduced pressure and the residue was purified by column chromatography on silica gel to afford the dienol S7.

A round bottom flask containing $\mathrm{LiCl}(630 \mathrm{mg}, 15 \mathrm{mmol})$ was flamed dried three times under high vacuum and charged with $\mathrm{Pd}\left(\mathrm{PPh}_{3}\right)_{4}(173 \mathrm{mg}, 0.15 \mathrm{~mol})$ and $\mathrm{CuCl}$ (1.78 $\mathrm{g}, 18 \mathrm{mmol})$. The reaction flask was degassed three times under high vacuum and purged with argon. To the solid was added DMSO $(8 \mathrm{~mL})$, dienol S7 (1.38 g, 3 mmol), halide ( $3 \mathrm{mmol})$. The black reaction mixture was purged with Ar for $20 \mathrm{~min}$. The reaction was heated at $60{ }^{\circ} \mathrm{C}$ for $20 \mathrm{~h}$ and then cooled to rt. The reaction mixture was diluted with $10 \mathrm{~mL}$ of $\mathrm{Et}_{2} \mathrm{O}$ and washed with brine. The aqueous layer was then extracted with $\mathrm{Et}_{2} \mathrm{O}$. The combined organic layers were washed with water, dried, and concentrated. The residue was purified by column chromatography on silica gel to afford the desired dienol S8, which subsequently was oxidized to corresponding dienone 1 according the similar procedure described in Route A. 


\section{Route D 错误!末定义书签。}<smiles>[R]C(C=O)=CCCCCC(C)=CCCC</smiles><smiles>[R]C(=C[As])C(O)C(=C)C</smiles>

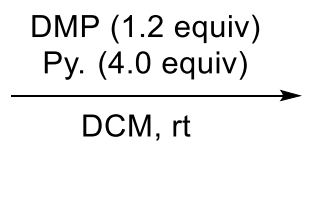<smiles>[R]C(=C[13CH3])C(=O)C(=C)[13CH3]</smiles>

The isopropenylmagnesium bromide $(12 \mathrm{~mL}, 6 \mathrm{mmol})$ was added dropwise to a solution of substituted acrolein $\mathbf{S 9}(5 \mathrm{mmol})$ in $\mathrm{Et}_{2} \mathrm{O}(20 \mathrm{~mL})$. The reaction mixture was stirred for $30 \mathrm{~min}$ at rt. Saturated aqueous $\mathrm{NH}_{4} \mathrm{Cl}(10 \mathrm{~mL})$ was added to quench the reaction. The resulting mixture was then extracted with $\mathrm{Et}_{2} \mathrm{O}$ and the organic layer was combined and dried over anhydrous $\mathrm{MgSO}_{4}$. The solvent was evaporated under reduced pressure and the residue was purified by column chromatography on silica gel to afford the desired dienol S10, which subsequently was oxidized to corresponding dienone $\mathbf{1}$ according the similar procedure described in Route A.

\section{Route E}

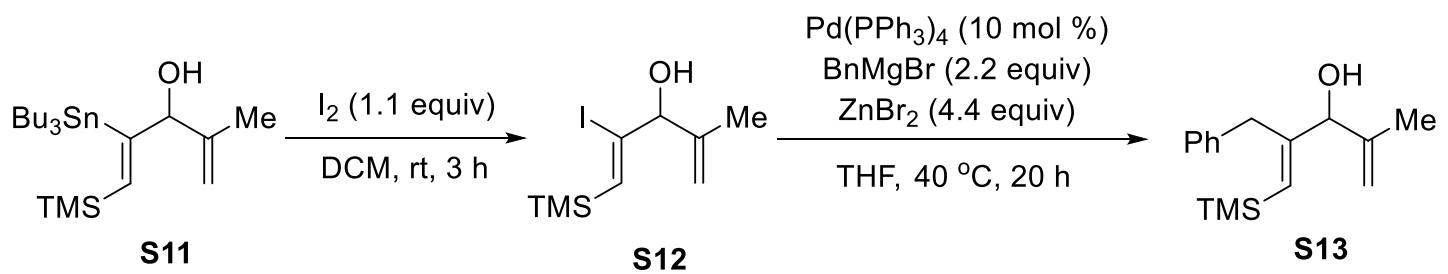<smiles>C=C(C)C(=O)C(=C[Sn])Cc1ccccc1</smiles>

To a stirred solution of stannol S11 (8 $\mathrm{g}, 17.4 \mathrm{mmol})$, which was prepared through the method described in Route C, in DCM (300 mL) was added $50 \mathrm{~mL}$ DCM solution of $\mathrm{I}_{2}(4.86 \mathrm{~g}, 19.1 \mathrm{mmol})$ and the dark solution was vigorously stirred at $\mathrm{rt}$ for $3 \mathrm{~h}$. Saturated aqueous $\mathrm{Na}_{2} \mathrm{~S}_{2} \mathrm{O}_{3}$ was added and the phases were separated. The aueous phase was extracted with DCM. The combined organic phases were dried over $\mathrm{Na}_{2} \mathrm{SO}_{4}$ and concentrated. the residue was purified by column chromatography on silica gel to afford the iododienol S12. 
A solution of anhydrous zinc bromide $(1.5 \mathrm{~g}, 6.6 \mathrm{mmol})$ in anhydrous THF (10 $\mathrm{mL})$ was cooled to $0{ }^{\circ} \mathrm{C}$ and benzylmagnesium bromide $(3.3 \mathrm{~mL}$ of a $1 \mathrm{M}$ THF solution, $3.3 \mathrm{mmol}$ ) was added dropwise. The mixture was stirred at $0{ }^{\circ} \mathrm{C}$ for $1 \mathrm{~h}$ and iododienol S12 (444 mg, $1.5 \mathrm{mmol})$ and $\mathrm{Pd}\left(\mathrm{PPh}_{3}\right)_{4}(173 \mathrm{mg}, 0.15 \mathrm{mmol})$ were added. The mixture was stirred at $40{ }^{\circ} \mathrm{C}$ for $20 \mathrm{~h}$. Saturated aqueous $\mathrm{NH}_{4} \mathrm{Cl}$ was added to quench the reaction. The resulting mixture was then extracted with ethyl acetate and the organic layer was combined and dried over anhydrous $\mathrm{MgSO}_{4}$. The solvent was evaporated under reduced pressure and the residue was purified by column chromatography on silica gel to afford the desired dienol S13, which subsequently been oxidized to corresponding dienone 1da using the method described in Route A.

\section{Route F}

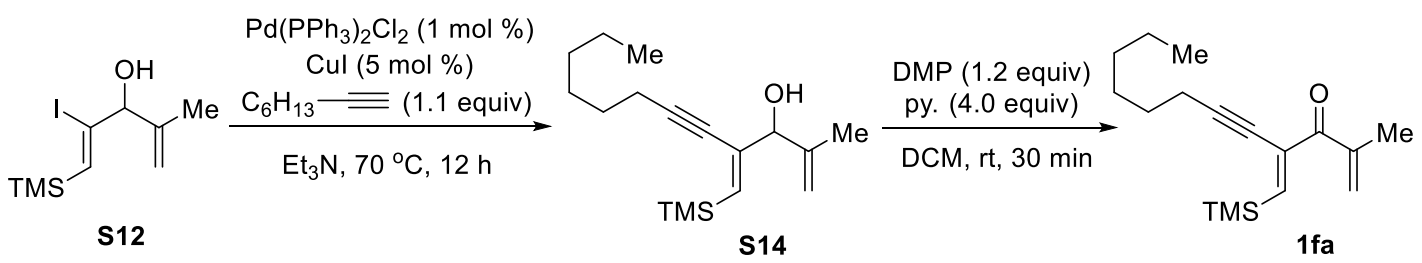

To a $50 \mathrm{~mL}$ Schlenk flask were added $\mathrm{Pd}\left(\mathrm{PPh}_{3}\right)_{2} \mathrm{Cl}_{2}(11 \mathrm{mg}, 0.015 \mathrm{mmol}), \mathrm{CuI}$ (14 mg, $0.075 \mathrm{mmol})$ under Ar. To the solid was added $\mathrm{Et}_{3} \mathrm{~N}(10 \mathrm{~mL})$, iododienol S12 (444 mg, $1.5 \mathrm{mmol}$, prepared using the method described in Route E), 1-octyne (182 $\mathrm{mg}, 1.65 \mathrm{mmol})$. The reaction was heated at $70{ }^{\circ} \mathrm{C}$ for $12 \mathrm{~h}$ and then cooled to $\mathrm{rt}$. The mixture was then concentrated under reduced pressure and the residue was purified by column chromatography on silica gel to afford the desired dienol S14, which subsequently been oxidized to corresponding dienone 1fa using the method described in Route A. 


\section{Route G}

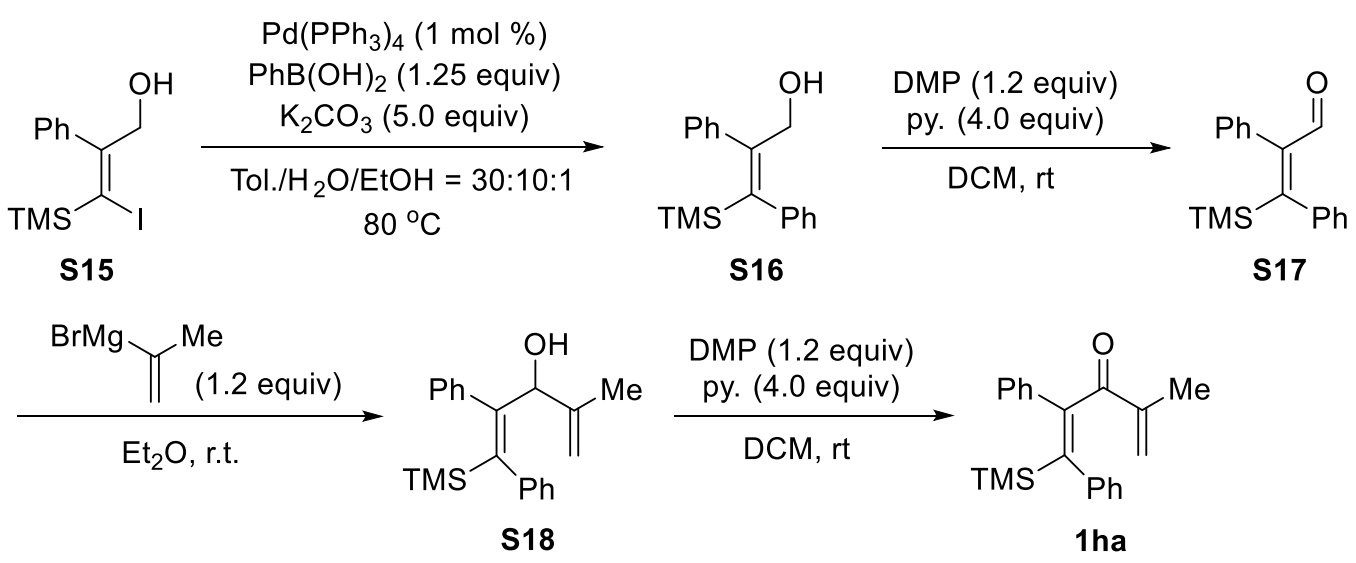

In an oven dried $100 \mathrm{~mL}$ three-necked flask, $\mathrm{Pd}\left(\mathrm{PPh}_{3}\right)_{4}(58 \mathrm{mg}, 0.05 \mathrm{mmol})$, $\mathrm{PhB}(\mathrm{OH})_{2}$ (762 mg, $\left.6.25 \mathrm{mmol}\right), \mathrm{K}_{2} \mathrm{CO}_{3}(3.45 \mathrm{~g}, 25 \mathrm{mmol})$ were added successively. Then iodoenol S15 (1.66 g, $5 \mathrm{mmol})$, toluene $(30 \mathrm{~mL}), \mathrm{H}_{2} \mathrm{O}(10 \mathrm{~mL})$, EtOH $(1 \mathrm{~mL})$ were added to the former suspension under Ar. The resulting mixture was stirred at reflux for $48 \mathrm{~h}$, then concentrated under reduced pressure and the residue was purified by column chromatography on silica gel to afford the desired allyl alcohol S16. Following processes are consistent with Route D to afford 1 ha.

\section{Route H}

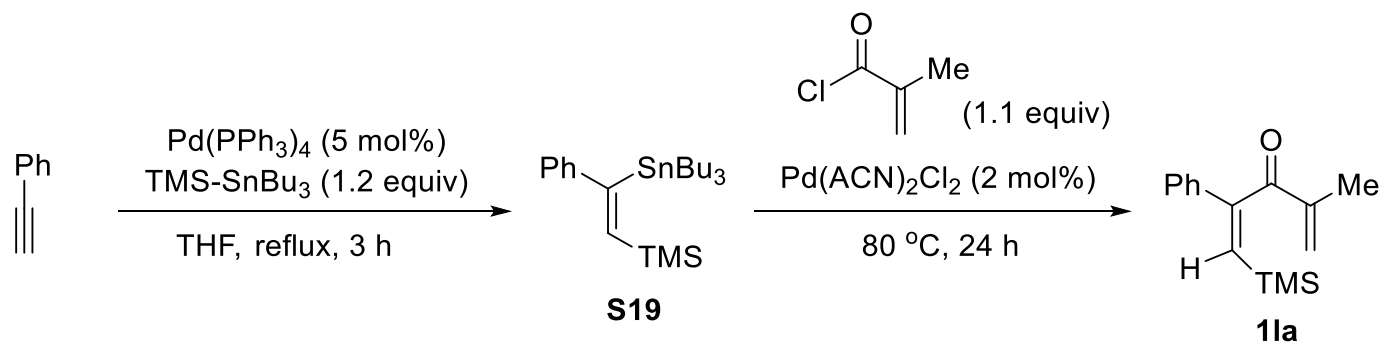

In a dried, three-necked flask fitted with a reflux condenser and Ar inlet, TMS-SnBu $3(1.74 \mathrm{~g}, 4.8 \mathrm{mmol})$, phenylacetylene (410 mg, $4 \mathrm{mmol})$, and $\mathrm{Pd}\left(\mathrm{PPh}_{3}\right)_{4}$ (231 mg, $0.2 \mathrm{mmol})$ are dissolved in THF $(10 \mathrm{~mL})$ and refluxed for $3 \mathrm{~h}$. The solution is concentrated, diluted with $\mathrm{Et}_{2} \mathrm{O}$, and filtered over Celite. After evaporation, the residue is purified by distillation $\left(0.12\right.$ tor, b.p.: $\left.125^{\circ} \mathrm{C}\right)$ to give the desired tin reagent S19.

Then to a $10 \mathrm{~mL}$ Schlenk tube were added tin reagent $\mathbf{S 1 9}(1.39 \mathrm{~g}, 3 \mathrm{mmol})$, methacryloyl chloride (343 mg, $3.3 \mathrm{mmol}), \mathrm{Pd}(\mathrm{ACN})_{2} \mathrm{Cl}_{2}(16 \mathrm{mg}, 0.06 \mathrm{mmol})$ under 
Ar. The reaction was heated at $80{ }^{\circ} \mathrm{C}$ for $24 \mathrm{~h}$. The mixture was then concentrated under reduced pressure and the residue was purified by column chromatography on silica gel to afford the desired dienone 1la. 


\subsection{Analytical data of substituted dienones}

\section{(E)-4-methyl-2-phenyl-1-(trimethylsilyl)penta-1,4-dien-3-one (1aa)}<smiles>C=C(C)C(=O)/C(=C/C#N)c1ccccc1</smiles>

Serial number: cj-1-61, Route A was followed with corresponding dienol S4 (1.2 g, $4.9 \mathrm{mmol})$, DMP (3.1 g, $7.3 \mathrm{mmol})$, pyridine (1.5 g, $19.5 \mathrm{mmol})$ in DCM (25 $\mathrm{mL})$ to afford 1aa as colorless oil (1.18 g, 98\% yield for the last step). TLC $R_{f}=0.79$ (petroleum ether/ethyl acetate, $\mathrm{PE} / \mathrm{EA}=10: 1, \mathrm{v} / \mathrm{v}$ ).

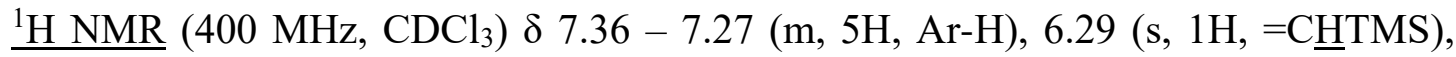
$5.92\left(\mathrm{~s}, 1 \mathrm{H},=\mathrm{CH}_{2}\right), 5.87\left(\mathrm{~d}, J=1.4 \mathrm{~Hz}, 1 \mathrm{H},=\mathrm{CH}_{2}\right), 1.97(\mathrm{~d}, J=1.3 \mathrm{~Hz}, 3 \mathrm{H}$, $\left.\mathrm{C}_{3}\right),-0.04$ (s, 9H, Si $\left.\underline{\mathrm{Me}}_{3}\right)$.

${ }^{13} \mathrm{C} \mathrm{NMR}\left(101 \mathrm{MHz}, \mathrm{CDCl}_{3}\right) \delta 199.6,155.6,143.9,139.2,139.0,129.0,128.2,128.2$, $127.8,18.3,0.0$.

HRMS-EI (m/z): calcd for [ $\left.\mathrm{C}_{15} \mathrm{H}_{20} \mathrm{OSi}, \mathrm{M}\right]^{+}:$244.1283, Found: 244.1282.

(E)-2-(4-methoxyphenyl)-4-methyl-1-(trimethylsilyl)penta-1,4-dien-3-one (1ab)<smiles>C=C(C)C(=O)C(=C[As])c1ccc(OC)cc1</smiles>

Serial number: cj-1-114, Route A was followed with corresponding dienol S4 (300 mg, $1.1 \mathrm{mmol})$, DMP (691 mg, $1.6 \mathrm{mmol})$, pyridine (344 mg, $4.4 \mathrm{mmol})$ in DCM (20 mL) to afford $1 \mathbf{a b}$ as colorless oil (270 mg, 90\% yield for the last step). TLC $R_{f}=0.70$ $(\mathrm{PE} / \mathrm{EA}=10: 1, \mathrm{v} / \mathrm{v})$.

$\underline{{ }^{1} \mathrm{H} \text { NMR }}\left(400 \mathrm{MHz}, \mathrm{CDCl}_{3}\right) \delta 7.19(\mathrm{~d}, J=8.6 \mathrm{~Hz}, 2 \mathrm{H}, \mathrm{Ar}-\mathrm{H}), 6.86(\mathrm{~d}, J=8.6 \mathrm{~Hz}, 2 \mathrm{H}$, Ar-H), $6.18(\mathrm{~s}, 1 \mathrm{H},=$ C $\underline{H} T M S), 5.87\left(\mathrm{~s}, 1 \mathrm{H},=\underline{\mathrm{C}}_{2}\right), 5.82(\mathrm{~d}, J=2.0 \mathrm{~Hz}, 1 \mathrm{H}$, $\left.=\underline{\mathrm{CH}}_{2}\right), 3.81(\mathrm{~s}, 3 \mathrm{H}, \underline{\mathrm{OMe}}), 1.93\left(\mathrm{~d}, J=1.3 \mathrm{~Hz}, 3 \mathrm{H}, \mathrm{C}_{3}\right),-0.04(\mathrm{~s}, 9 \mathrm{H}$, $\left.\mathrm{SiMe}_{3}\right)$. 
${ }^{13} \mathrm{C} \mathrm{NMR}\left(101 \mathrm{MHz}, \mathrm{CDCl}_{3}\right) \delta 199.9,159.6,155.2,143.8,138.1,130.0,127.6,113.6$, $55.4,55.4,18.2,0.0$.

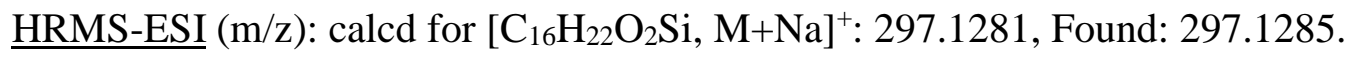

\section{(E)-4-methyl-2-(p-tolyl)-1-(trimethylsilyl)penta-1,4-dien-3-one (1ac)}<smiles>C=C(C)C(=O)C(=C[As])c1ccc(C)cc1</smiles>

Serial number: cj-2-28, Route A was followed with corresponding dienol S4 (520 mg, $2.0 \mathrm{mmol}$ ), DMP (1.0 g, $2.4 \mathrm{mmol})$, pyridine (640 mg, $8.0 \mathrm{mmol})$ in DCM (20 mL) to afford 1ac as colorless oil (410 mg, 80\% yield for the last step), TLC $R_{f}=0.82$ $(\mathrm{PE} / \mathrm{EA}=10: 1, \mathrm{v} / \mathrm{v})$.

${ }^{1} \mathrm{H} \mathrm{NMR}\left(400 \mathrm{MHz}, \mathrm{CDCl}_{3}\right) \delta 7.14(\mathrm{~d}, J=1.4 \mathrm{~Hz}, 4 \mathrm{H}, \mathrm{Ar}-\mathrm{H}), 6.20$ (s, 1H, =C $\underline{\mathrm{HTMS}}$ ), $5.90-5.87\left(\mathrm{~m}, 1 \mathrm{H},=\underline{\mathrm{C}}_{2}\right), 5.83\left(\mathrm{t}, J=1.5 \mathrm{~Hz}, 1 \mathrm{H},=\mathrm{C}_{2}\right), 2.35(\mathrm{~s}, 3 \mathrm{H}$, $\left.\mathrm{ArC}_{3}\right), 1.93$ (t, $\left.J=1.2 \mathrm{~Hz}, 3 \mathrm{H}, \mathrm{CH}_{3}\right),-0.06$ (s, 9H, SiMe $\left.\underline{\mathrm{M}}_{3}\right)$.

${ }^{13} \mathrm{C} \mathrm{NMR}\left(101 \mathrm{MHz}, \mathrm{CDCl}_{3}\right) \delta 199.9,155.6,143.8,138.4,137.9,135.9,128.9,128.7$, $127.8,21.5,18.2,0.0$.

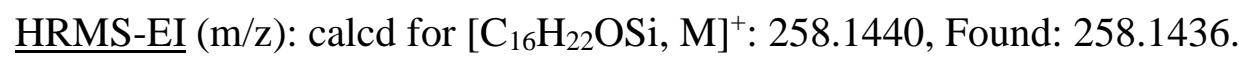

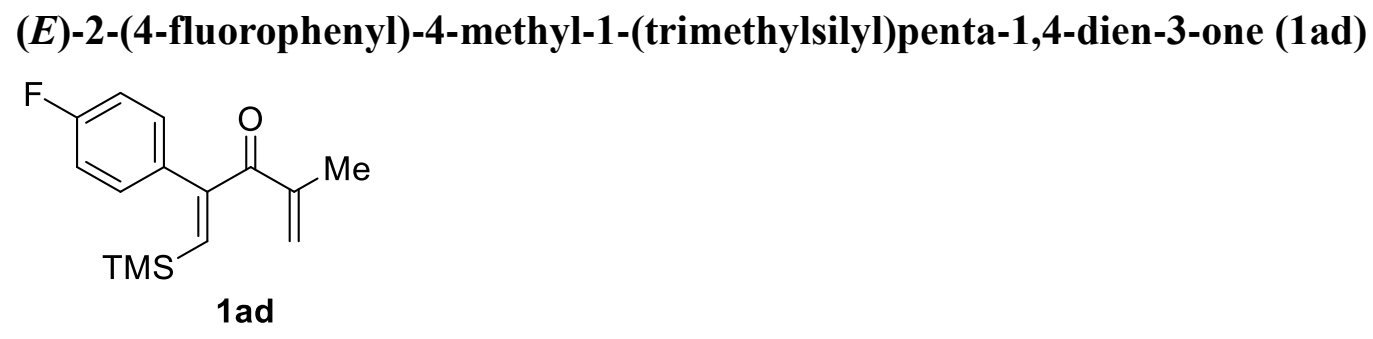

Serial number: cj-2-54, Route A was followed with corresponding dienol S4 (522 mg, $2.0 \mathrm{mmol})$, DMP (1.0 g, $2.4 \mathrm{mmol})$, pyridine (626 mg, $7.9 \mathrm{mmol})$ in DCM (25 mL) to afford 1ad as yellow oil (428 mg, 82\% yield for the last step). TLC $R_{f}=0.72$ (PE/EA $=10: 1, \mathrm{v} / \mathrm{v})$.

래 NMR $\left(400 \mathrm{MHz}, \mathrm{CDCl}_{3}\right) \delta 7.27-7.20(\mathrm{~m}, 2 \mathrm{H}, \mathrm{Ar}-\mathrm{H}), 7.02(\mathrm{t}, J=8.6 \mathrm{~Hz}, 2 \mathrm{H}$, $\mathrm{Ar}-\mathrm{H}), 6.30(\mathrm{~s}, 1 \mathrm{H},=\mathrm{C} \underline{\mathrm{H} T M S}), 5.86\left(\mathrm{~s}, 1 \mathrm{H},=\underline{\mathrm{CH}}_{2}\right), 5.85\left(\mathrm{~s}, 1 \mathrm{H},=\underline{\mathrm{CH}}_{2}\right), 1.95$ (s, 3H, $\left.\underline{\mathrm{H}}_{3}\right),-0.06$ (s, 9H, SiMe 3 ). 
${ }^{13} \mathrm{C}$ NMR $\left(101 \mathrm{MHz}, \mathrm{CDCl}_{3}\right) \delta 199.5,162.9(\mathrm{~d}, J=247.4 \mathrm{~Hz}), 154.3,143.8,140.3$, $134.9(\mathrm{~d}, J=3.0 \mathrm{~Hz}), 130.8$ (d, $J=8.1 \mathrm{~Hz}), 127.7,115.2(\mathrm{~d}, J=21.2 \mathrm{~Hz})$, 18.4, 0.0.

HRMS-EI (m/z): calcd for $\left[\mathrm{C}_{15} \mathrm{H}_{19} \mathrm{FOSi}, \mathrm{M}\right]^{+}: 262.1189$, Found: 262.1186.

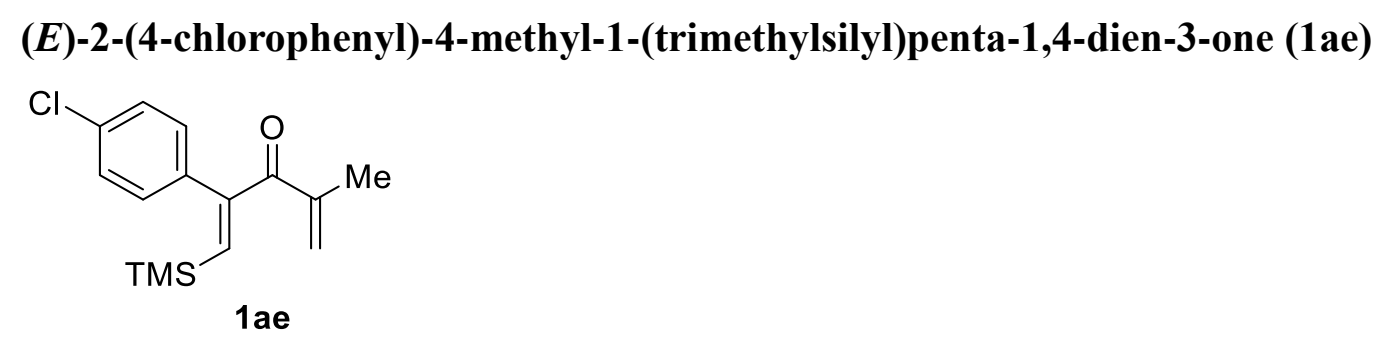

Serial number: cj-1-149, Route A was followed with corresponding dienol S4 (930 mg, $3.3 \mathrm{mmol})$, DMP (1.7 g, $4.0 \mathrm{mmol})$, pyridine $(1.0 \mathrm{~g}, 13.2 \mathrm{mmol})$ in DCM $(30 \mathrm{~mL})$ to afford 1ae as yellow oil (767 mg, 84\% yield for the last step). TLC $R_{f}=0.73$ (PE/EA $=10: 1, \mathrm{v} / \mathrm{v})$.

$\underline{{ }^{1} \mathrm{H} \mathrm{NMR}}\left(400 \mathrm{MHz}, \mathrm{CDCl}_{3}\right) \delta 7.35-7.27(\mathrm{~m}, 2 \mathrm{H}, \mathrm{Ar}-\mathrm{H}), 7.24-7.14(\mathrm{~m}, 2 \mathrm{H}, \mathrm{Ar}-\mathrm{H})$, $6.32(\mathrm{~s}, 1 \mathrm{H},=\mathrm{C} \underline{\mathrm{HTMS}}), 5.85\left(\mathrm{~m}, 2 \mathrm{H},=\mathrm{C}_{2}\right), 1.94\left(\mathrm{t}, J=1.2 \mathrm{~Hz}, 3 \mathrm{H}, \mathrm{C}_{3}\right)$, -0.06 (s, 9H, $\left.\mathrm{SiMe}_{3}\right)$.

${ }^{13} \mathrm{C} \mathrm{NMR}\left(101 \mathrm{MHz}, \mathrm{CDCl}_{3}\right) \delta 199.6,154.6,144.2,141.0,137.8,134.7,130.8,128.9$, $128.1,18.7,0.4$.

HRMS-EI (m/z): calcd for $\left[\mathrm{C}_{15} \mathrm{H}_{19} \mathrm{ClOSi}, \mathrm{M}\right]^{+}: 278.0894$, Found: 278.0890 .

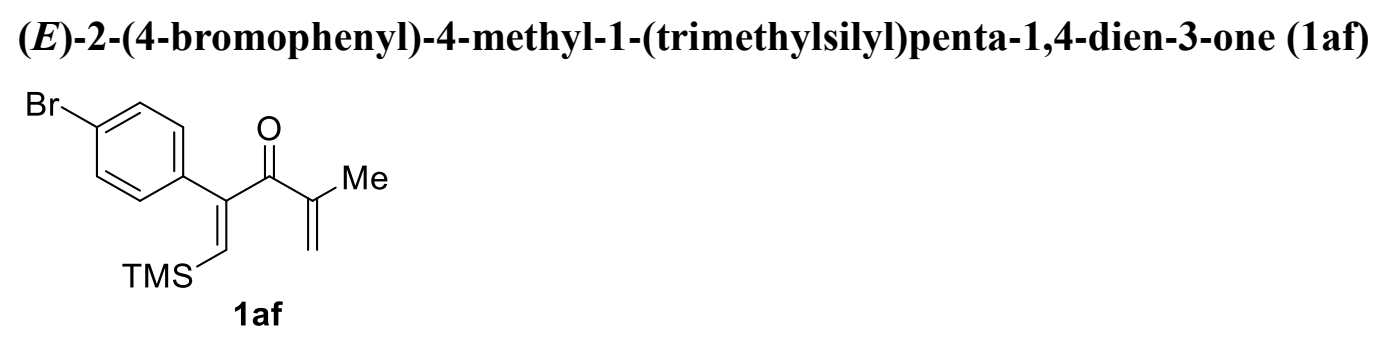

Serial number: cj-2-53, Route A was followed with corresponding dienol S4 (544 mg, $1.7 \mathrm{mmol})$, DMP (854 mg, $2.0 \mathrm{mmol})$, pyridine (531 mg, $6.7 \mathrm{mmol})$ in DCM (20 mL) to afford 1af as yellow oil ( $462 \mathrm{mg}, 85 \%$ yield for the last step). TLC $R_{f}=0.69$ $(\mathrm{PE} / \mathrm{EA}=10: 1, \mathrm{v} / \mathrm{v})$.

吕 NMR $\left(400 \mathrm{MHz}, \mathrm{CDCl}_{3}\right) \delta 7.51-7.44(\mathrm{~m}, 2 \mathrm{H}, \mathrm{Ar}-\mathrm{H}), 7.14(\mathrm{~d}, J=8.4 \mathrm{~Hz}, 2 \mathrm{H}$, Ar-H), $6.32(\mathrm{~s}, 1 \mathrm{H},=$ C TMS $), 5.87-5.83\left(\mathrm{~m}, 2 \mathrm{H},=\underline{\mathrm{C}}_{2}\right), 1.94(\mathrm{~s}, 3 \mathrm{H}$, 
$\left.\mathrm{C}_{3}\right),-0.06$ (s, 9H, $\left.\mathrm{SiMe}_{3}\right)$.

${ }^{13} \mathrm{C} \mathrm{NMR}\left(101 \mathrm{MHz}, \mathrm{CDCl}_{3}\right) \delta 199.2,154.1,143.7,140.6,137.8,131.4,130.7,127.8$, $122.4,18.3,0.0$.

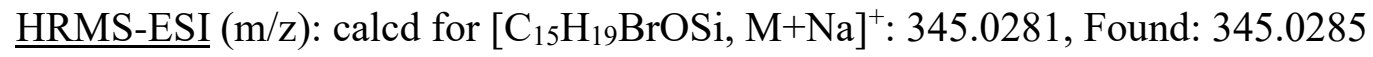

(E)-4-methyl-2-(4-(trifluoromethyl)phenyl)-1-(trimethylsilyl)penta-1,4-dien-3-on e (1ag)<smiles>C=C(C)C(=O)C(=C[As])c1ccc(C(F)(F)F)cc1</smiles>

Serial number: cj-3-43, Route C was followed with corresponding dienol S8 (725 mg, $2.3 \mathrm{mmol})$, DMP (1.5 g, $3.5 \mathrm{mmol})$ in DCM (30 mL) to afford 1ag as colorless oil (671 mg, 93\% yield for the last step). TLC $R_{f}=0.71(\mathrm{PE} / \mathrm{EA}=10: 1, \mathrm{v} / \mathrm{v})$.

$\underline{{ }^{1} \mathrm{H} \text { NMR }}\left(400 \mathrm{MHz}, \mathrm{CDCl}_{3}\right) \delta 7.60(\mathrm{dd}, J=8.3,2.2 \mathrm{~Hz}, 2 \mathrm{H}, \mathrm{Ar}-\mathrm{H}), 7.38(\mathrm{dd}, J=8.2$, $2.2 \mathrm{~Hz}, 2 \mathrm{H}, \mathrm{Ar}-\mathrm{H}), 6.41$ (d, $J=2.3 \mathrm{~Hz}, 1 \mathrm{H},=$ C $\underline{\mathrm{HTMS}}), 5.91-5.85$ (m, 2H, $\left.=\underline{\mathrm{CH}}_{2}\right), 1.96\left(\mathrm{dd}, J=2.7,1.4 \mathrm{~Hz}, 3 \mathrm{H}, \underline{\mathrm{C}}_{3}\right),-0.07$ (s, 9H, SiMe 3 ).

${ }^{13} \mathrm{C} \mathrm{NMR}\left(101 \mathrm{MHz}, \mathrm{CDCl}_{3}\right) \delta 199.0,154.0,143.8,142.7,141.8,130.42$ (q, $J=32.3$ $\mathrm{Hz}), 129.6,127.9,125.3(\mathrm{q}, J=4.0 \mathrm{~Hz}), 124.5(\mathrm{q}, J=272.7 \mathrm{~Hz}), 18.4,0.00$.

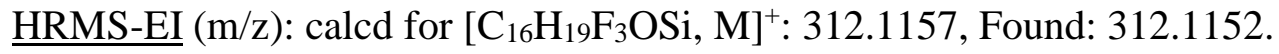

\section{(E)-4-methyl-2-(m-tolyl)-1-(trimethylsilyl)penta-1,4-dien-3-one (1ah)}<smiles>C=C(C)C(=O)C(=C[As])c1cccc(C)c1</smiles>

Serial number: cj-2-29, Route A was followed with corresponding dienol S4 (650 mg, $2.5 \mathrm{mmol}$ ), DMP (1.3 g, $3 \mathrm{mmol})$, pyridine (790 mg, $10.0 \mathrm{mmol})$ in DCM (25 mL) to afford 1ah as colorless oil (550 mg, 85\% yield for the last step). TLC $R_{f}=0.81$ $(\mathrm{PE} / \mathrm{EA}=10: 1, \mathrm{v} / \mathrm{v})$. 
$\underline{{ }^{1} \mathrm{H} \mathrm{NMR}}\left(400 \mathrm{MHz}, \mathrm{CDCl}_{3}\right) \delta 7.20(\mathrm{~d}, J=7.5 \mathrm{~Hz}, 1 \mathrm{H}, \mathrm{Ar}-\mathrm{H}), 7.12(\mathrm{~d}, J=7.5 \mathrm{~Hz}, 1 \mathrm{H}$, Ar-H), $7.10-7.03$ (m, 2H, Ar-H), 6.21 (s, 1H, =CㅌTMS), 5.90 (s, 1H, $\left.=\underline{\mathrm{C}}_{2}\right), 5.84\left(\mathrm{~s}, 1 \mathrm{H},=\underline{\mathrm{C}}_{2}\right), 2.34\left(\mathrm{~s}, 3 \mathrm{H}, \mathrm{ArC}_{3}\right), 1.94\left(\mathrm{~s}, 3 \mathrm{H}, \mathrm{C}_{3}\right),-0.06(\mathrm{~s}$, 9H, $\left.\mathrm{SiMe}_{3}\right)$.

${ }^{13} \mathrm{C} \mathrm{NMR}\left(101 \mathrm{MHz}, \mathrm{CDCl}_{3}\right) \delta 199.8,155.8,143.8,138.8,138.7,137.7,129.6,128.9$, $128.1,127.9,126.0,21.6,18.2,0.0$.

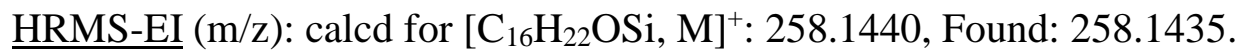

\section{(E)-4-methyl-2-(o-tolyl)-1-(trimethylsilyl)penta-1,4-dien-3-one (1ai)}<smiles>C=C(C)C(=O)/C(=C/C)c1ccccc1C</smiles>

1ai

Serial number: cj-3-42, Route C was followed with corresponding dienol S8 (647 mg, $2.5 \mathrm{mmol}$ ), DMP (1.6 g, $3.7 \mathrm{mmol})$ in DCM (30 mL) to afford 1ai as colorless oil (588 mg, 91\% yield for the last step). TLC $R_{f}=0.79(\mathrm{PE} / \mathrm{EA}=10: 1, \mathrm{v} / \mathrm{v})$.

${ }^{1} \mathrm{H}$ NMR $\left(400 \mathrm{MHz}, \mathrm{CDCl}_{3}\right) \delta 7.23-7.12(\mathrm{~m}, 3 \mathrm{H}, \mathrm{Ar}-\mathrm{H}), 7.05(\mathrm{~d}, J=7.6 \mathrm{~Hz}, 1 \mathrm{H}$, $\operatorname{Ar}-\mathrm{H}), 6.66(\mathrm{~s}, 1 \mathrm{H},=\mathrm{C} \underline{\mathrm{HT} M S}), 5.80\left(\mathrm{~s}, 1 \mathrm{H},=\underline{\mathrm{C}}_{2}\right), 5.77\left(\mathrm{~s}, 1 \mathrm{H},=\mathrm{C}_{2}\right), 2.18$

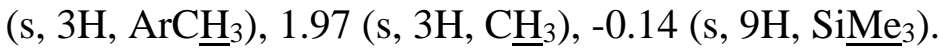

${ }^{13} \mathrm{C}$ NMR $\left(101 \mathrm{MHz}, \mathrm{CDCl}_{3}\right) \delta 199.3,155.6,144.7,144.5,139.5,136.9,131.1,130.7$, $128.9,126.2,126.1,21.0,19.8,0.0$.

HRMS-EI (m/z): calcd for $\left[\mathrm{C}_{16} \mathrm{H}_{22} \mathrm{OSi}, \mathrm{M}\right]^{+}:$258.1440, Found:258.1437.

\section{(E)-2-(benzo[d][1,3]dioxol-5-yl)-4-methyl-1-(trimethylsilyl)penta-1,4-dien-3-one}

(1aj)<smiles>C=C(C)C(=O)C(=C[As])c1ccc2c(c1)OCO2</smiles>

Serial number: cj-1-196, Route A was followed with corresponding dienol S4 (400 mg, $1.4 \mathrm{mmol}$ ), DMP (850 mg, $2.0 \mathrm{mmol})$, pyridine (442 mg, $5.6 \mathrm{mmol})$ in DCM (20 mL) 
to afford 1aj as yellow oil (352 $\mathrm{mg}, 87 \%$ yield for the last step). TLC $R_{f}=0.67$ $(\mathrm{PE} / \mathrm{EA}=10: 1, \mathrm{v} / \mathrm{v})$.

$\underline{{ }^{1} \mathrm{H} \mathrm{NMR}}\left(400 \mathrm{MHz}, \mathrm{CDCl}_{3}\right) \delta 6.80-6.75(\mathrm{~m}, 2 \mathrm{H}, \mathrm{Ar}-\mathrm{H}), 6.71(\mathrm{~m}, 1 \mathrm{H}, \mathrm{Ar}-\mathrm{H}), 6.18(\mathrm{~s}$, $1 \mathrm{H},=\mathrm{C} \underline{\mathrm{HT} M S}), 5.96\left(\mathrm{~s}, 2 \mathrm{H}, \mathrm{OC}_{2} \mathrm{O}\right), 5.86\left(\mathrm{~s}, 1 \mathrm{H},=\underline{\mathrm{C}}_{2}\right), 5.84(\mathrm{~s}, 1 \mathrm{H}$, $\left.=\underline{\mathrm{CH}}_{2}\right), 1.93\left(\mathrm{~s}, 3 \mathrm{H}, \underline{\mathrm{CH}}_{3}\right),-0.02\left(\mathrm{~s}, 9 \mathrm{H}, \mathrm{SiMe}_{3}\right)$.

${ }^{13} \mathrm{C} \mathrm{NMR}\left(101 \mathrm{MHz}, \mathrm{CDCl}_{3}\right) \delta 199.7,154.9,147.6,147.5,143.8,138.8,132.7,127.8$, 122.6, 109.4, 108.1, 101.3, 18.2, 0.0.

HRMS-EI (m/z): calcd for $\left[\mathrm{C}_{16} \mathrm{H}_{20} \mathrm{O}_{3} \mathrm{Si}, \mathrm{M}\right]^{+}:$288.1182, Found: 288.1176.

(E)-4-methyl-2-(naphthalen-2-yl)-1-(trimethylsilyl)penta-1,4-dien-3-one (1ak)<smiles>C=C(C)C(=O)C(=CC)c1ccc2ccccc2c1</smiles>

Serial number: cj-1-191, Route A was followed with corresponding dienol S4 (853 mg, $2.9 \mathrm{mmol})$, DMP (1.5 g, $3.5 \mathrm{mmol})$, pyridine (910 mg, $11.5 \mathrm{mmol})$ in DCM (30 mL) to afford 1ak as, yellow solid (783 mg, 93\% yield for the last step). mp: $46-47{ }^{\circ} \mathrm{C}$. TLC $R_{f}=0.72(\mathrm{PE} / \mathrm{EA}=10: 1, \mathrm{v} / \mathrm{v})$.

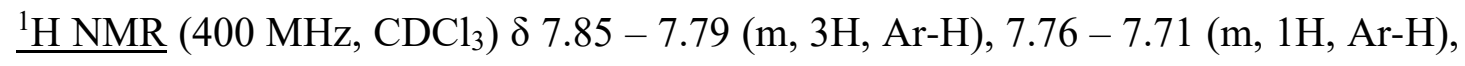
$7.48(\mathrm{~d}, J=9.4 \mathrm{~Hz}, 2 \mathrm{H}, \mathrm{Ar}-\mathrm{H}), 7.40$ (dd, $J=8.4,1.7 \mathrm{~Hz}, 1 \mathrm{H}, \mathrm{Ar}-\mathrm{H}), 6.35$ (s, $1 \mathrm{H},=\mathrm{C} \underline{\mathrm{H} T M S}), 5.97-5.95\left(\mathrm{~m}, 1 \mathrm{H},=\underline{\mathrm{C}}_{2}\right), 5.89-5.86\left(\mathrm{~m}, 1 \mathrm{H},=\underline{\mathrm{CH}}_{2}\right)$, $1.97\left(\mathrm{t}, J=1.2 \mathrm{~Hz}, 3 \mathrm{H}, \underline{\mathrm{CH}}_{3}\right),-0.06$ (s, 9H, $\left.\mathrm{SiMe}_{3}\right)$.

${ }^{13} \mathrm{C} \mathrm{NMR}\left(101 \mathrm{MHz}, \mathrm{CDCl}_{3}\right) \delta 199.6,155.6,143.8,139.5,136.4,133.1,133.0,128.2$, $127.9,127.9,127.9,127.8,126.9,126.4,126.4,18.2,0.0$.

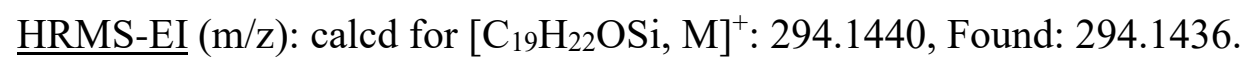


(Z)-4-methyl-2-(thiophen-2-yl)-1-(trimethylsilyl)penta-1,4-dien-3-one (1al)<smiles>C=C(C)C(=O)C(=CSC)c1cccs1</smiles>

Serial number: cj-1-154, Route A was followed with corresponding dienol S4 (600 mg, $2.4 \mathrm{mmol}$ ), DMP (1.2 g, $2.9 \mathrm{mmol})$, pyridine (760 mg, $9.6 \mathrm{mmol})$ in DCM (20 mL) to afford 1al as yellow oil (510 mg, 85\% yield for the last step). TLC $R_{f}=0.70$ (PE/EA $=$ $10: 1, \mathrm{v} / \mathrm{v})$.

를 NMR $\left(400 \mathrm{MHz}, \mathrm{CDCl}_{3}\right) \delta 7.32(\mathrm{dd}, J=4.8,1.5 \mathrm{~Hz}, 1 \mathrm{H}, \mathrm{Ar}-\mathrm{H}), 7.03-6.97(\mathrm{~m}$, 2H, Ar-H), $6.22(\mathrm{~s}, 1 \mathrm{H},=$ C $\underline{H} T M S), 5.92\left(\mathrm{t}, J=1.0 \mathrm{~Hz}, 1 \mathrm{H},=\mathrm{CH}_{2}\right), 5.88(\mathrm{~m}$, $\left.1 \mathrm{H},=\underline{\mathrm{C}}_{2}\right), 1.96\left(\mathrm{dd}, J=1.5,0.9 \mathrm{~Hz}, 3 \mathrm{H}, \underline{\mathrm{C}}_{3}\right), 0.05$ (s, 9H, SiMe 3 ).

${ }^{13} \mathrm{C} \mathrm{NMR}\left(101 \mathrm{MHz}, \mathrm{CDCl}_{3}\right) \delta 198.4,148.2,142.9,139.8,138.9,128.2,127.5,126.9$, $126.6,17.8,0.3$.

$\underline{\text { HRMS-EI }}(\mathrm{m} / \mathrm{z})$ : calcd for $\left[\mathrm{C}_{13} \mathrm{H}_{18} \mathrm{OSSi}, \mathrm{M}\right]^{+}:$250.0848, Found: 250.0844 .

\section{(E)-4-methylene-2-phenyl-1-(trimethylsilyl)hex-1-en-3-one (1ba)}<smiles>C=C(CC)C(=O)C(=C[13CH3])c1ccccc1</smiles>

Serial number: cj-2-49, Route A was followed with corresponding dienol S4 (800 mg, $3.1 \mathrm{mmol}$ ), DMP (1.6 g, $3.7 \mathrm{mmol})$, pyridine (970 mg, $12.3 \mathrm{mmol})$ in DCM (20 mL) to afford $1 \mathrm{ba}$ as colorless oil (548 $\mathrm{mg}, 69 \%$ yield for the last step). TLC $R_{f}=0.81$ $(\mathrm{PE} / \mathrm{EA}=10: 1, \mathrm{v} / \mathrm{v})$.

${ }^{1} \mathrm{H}$ NMR $\left(400 \mathrm{MHz}, \mathrm{CDCl}_{3}\right) \delta 7.36-7.27$ (m, 3H, Ar-H), $7.26-7.22$ (m, 2H, Ar-H), $6.29(\mathrm{~s}, 1 \mathrm{H},=\mathrm{C} \underline{\mathrm{HTMS}}), 5.86\left(\mathrm{~d}, J=1.1 \mathrm{~Hz}, 1 \mathrm{H},=\underline{\mathrm{C}}_{2}\right), 5.76(\mathrm{~d}, J=1.3 \mathrm{~Hz}$, $\left.1 \mathrm{H},=\underline{\mathrm{CH}}_{2}\right), 2.36\left(\mathrm{dt}, J=7.5,1.2 \mathrm{~Hz}, 2 \mathrm{H}, \underline{\mathrm{CH}}_{2} \mathrm{CH}_{3}\right), 1.06(\mathrm{t}, J=7.4 \mathrm{~Hz}, 3 \mathrm{H}$, $\left.\mathrm{C}_{3}\right),-0.07$ (s, 9H, $\left.\mathrm{SiMe}_{3}\right)$.

${ }^{13} \mathrm{C} \mathrm{NMR}\left(101 \mathrm{MHz}, \mathrm{CDCl}_{3}\right) \delta 199.7,156.0,149.8,139.8,139.0,129.1,128.2,128.2$, $125.4,24.8,12.7,0.0$. 


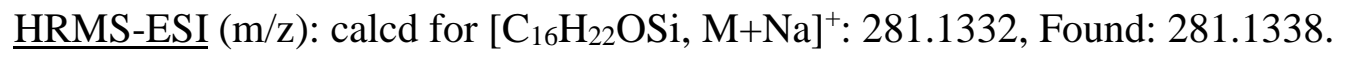

\section{(E)-4-methylene-2-phenyl-1-(trimethylsilyl)hept-1-en-3-one (1bb)}<smiles></smiles>

Serial number: cj-2-50, Route A was followed with corresponding dienol S4 (280 mg, $1.0 \mathrm{mmol}$ ), DMP (520 mg, $1.2 \mathrm{mmol})$, pyridine (322 mg, $4.1 \mathrm{mmol})$ in DCM (20 mL) to afford $\mathbf{1 b b}$ as colorless oil (207 $\mathrm{mg}, 74 \%$ yield for the last step). TLC $R_{f}=0.83$ $(\mathrm{PE} / \mathrm{EA}=10: 1, \mathrm{v} / \mathrm{v})$.

${ }^{1} \mathrm{H}$ NMR $\left(400 \mathrm{MHz}, \mathrm{CDCl}_{3}\right) \delta 7.32(\mathrm{~d}, J=6.6 \mathrm{~Hz}, 3 \mathrm{H}, \mathrm{Ar}-\mathrm{H}), 7.24(\mathrm{dd}, J=7.2,2.4$ $\mathrm{Hz}, 2 \mathrm{H}, \mathrm{Ar}-\mathrm{H}), 6.29(\mathrm{~s}, 1 \mathrm{H},=\mathrm{C} \underline{\mathrm{HTMS}}), 5.86\left(\mathrm{~s}, 1 \mathrm{H},=\mathrm{C}_{2}\right), 5.78-5.74(\mathrm{~m}$, $\left.1 \mathrm{H},=\underline{\mathrm{C}}_{2}\right), 2.31\left(\mathrm{t}, J=7.6 \mathrm{~Hz}, 2 \mathrm{H}, \mathrm{C}_{2} \mathrm{CH}_{2} \mathrm{CH}_{3}\right), 1.47(\mathrm{~m}, 2 \mathrm{H}$, $\mathrm{CH}_{2} \underline{\mathrm{C}}_{2} \mathrm{CH}_{3}$ ), 0.92 (t, $\left.J=7.3 \mathrm{~Hz}, 3 \mathrm{H}, \underline{\mathrm{CH}}_{3}\right),-0.07$ (s, 9H, SiMe 3 ).

${ }^{13} \mathrm{C} \mathrm{NMR}\left(101 \mathrm{MHz}, \mathrm{CDCl}_{3}\right) \delta 199.6,156.1,148.3,139.8,139.0,129.1,128.2,128.2$, $126.3,33.9,21.7,14.1,0.0$.

HRMS-EI (m/z): calcd for [ $\left.\mathrm{C}_{17} \mathrm{H}_{24} \mathrm{OSi}, \mathrm{M}\right]^{+}:$272.1596, Found: 272.1593.

\section{(E)-4-methylene-2-phenyl-1-(trimethylsilyl)dec-1-en-3-one (1bc)}<smiles>C=C(CCCCCC)C(=O)C(=C[13CH3])c1ccccc1</smiles>

Serial number: cj-1-130, Route A was followed with corresponding dienol S4 (390 mg, $1.2 \mathrm{mmol}$ ), DMP (785 mg, $1.8 \mathrm{mmol})$, pyridine (389 mg, $5.0 \mathrm{mmol})$ in DCM (15 $\mathrm{mL}$ ) to afford $\mathbf{1 b c}$ as colorless oil (330 $\mathrm{mg}, 85 \%$ yield for the last step). TLC $R_{f}=0.91$ $(\mathrm{PE} / \mathrm{EA}=10: 1, \mathrm{v} / \mathrm{v})$.

$\underline{{ }^{1} \mathrm{H} \mathrm{NMR}}\left(400 \mathrm{MHz}, \mathrm{CDCl}_{3}\right) \delta 7.32(\mathrm{~d}, J=6.6 \mathrm{~Hz}, 3 \mathrm{H}, \mathrm{Ar}-\mathrm{H}), 7.27-7.21(\mathrm{~m}, 2 \mathrm{H}$, Ar-H), $6.29(\mathrm{~s}, 1 \mathrm{H},=\mathrm{C} \underline{\mathrm{HT} M S}), 5.84\left(\mathrm{~s}, 1 \mathrm{H},=\underline{\mathrm{C}}_{2}\right), 5.75\left(\mathrm{~s}, 1 \mathrm{H},=\underline{\mathrm{C}}_{2}\right), 2.32$ $\left(\mathrm{t}, J=7.6 \mathrm{~Hz}, 2 \mathrm{H}, \underline{\mathrm{C}}_{2} \mathrm{C}_{5} \mathrm{H}_{11}\right), 1.46-1.39\left(\mathrm{~m}, 2 \mathrm{H}, \mathrm{CH}_{2} \underline{\mathrm{C}}_{2} \mathrm{C}_{4} \mathrm{H}_{9}\right), 1.26(\mathrm{~m}$, 
$\left.6 \mathrm{H}, \mathrm{CH}_{2} \mathrm{CH}_{2}\left(\underline{\mathrm{C}}_{2}\right)_{3} \mathrm{CH}_{3}\right), 0.92-0.85\left(\mathrm{~m}, 3 \mathrm{H},\left(\mathrm{CH}_{2}\right)_{5} \mathrm{C}_{3}\right),-0.08(\mathrm{~s}, 9 \mathrm{H}$, $\left.\mathrm{SiMe}_{3}\right)$.

${ }^{13} \mathrm{C} \mathrm{NMR}\left(101 \mathrm{MHz}, \mathrm{CDCl}_{3}\right) \delta 199.7,156.1,148.6,139.9,139.0,129.1,128.2,128.2$, $126.2,32.0,31.9,29.3,28.4,22.9,14.4,0.0$.

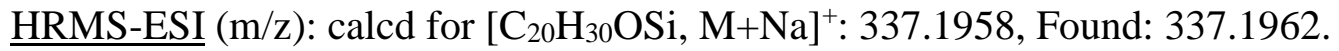

(E)-5-methyl-4-methylene-2-phenyl-1-(trimethylsilyl)hex-1-en-3-one (1bd)<smiles>C=C(C(=O)C(=C[13CH3])c1ccccc1)C(C)C</smiles>

Serial number: cj-2-189, Route A was followed with corresponding dienol S4 (700 mg, $2.5 \mathrm{mmol})$, DMP (1.6 g, $3.8 \mathrm{mmol})$, pyridine $(790 \mathrm{mg}, 10.0 \mathrm{mmol})$ in DCM (25 $\mathrm{mL})$ to afford $\mathbf{1 b d}$ as colorless oil (614 $\mathrm{mg}, 90 \%$ yield for the last step). TLC $R_{f}=$ $0.83(\mathrm{PE} / \mathrm{EA}=10: 1, \mathrm{v} / \mathrm{v})$.

${ }^{1} \mathrm{H}$ NMR $\left(400 \mathrm{MHz}, \mathrm{CDCl}_{3}\right) \delta 7.32(\mathrm{~m}, 3 \mathrm{H}, \mathrm{Ar}-\mathrm{H}), 7.26-7.21$ (m, 2H, Ar-H), 6.33 (s, $1 \mathrm{H},=\mathrm{CHTMS}), 5.79\left(\mathrm{~s}, 1 \mathrm{H},=\underline{\mathrm{C}}_{2}\right), 5.71-5.68\left(\mathrm{~m}, 1 \mathrm{H},=\mathrm{CH}_{2}\right), 2.98-2.87$

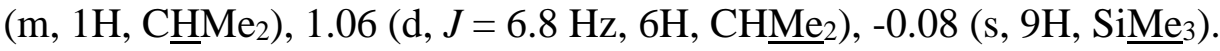

${ }^{13} \mathrm{C} \mathrm{NMR}\left(101 \mathrm{MHz}, \mathrm{CDCl}_{3}\right) \delta 199.9,156.5,154.6,140.6,139.0,129.2,128.2,128.2$, 123.0, 29.1, 21.8, 0.0.

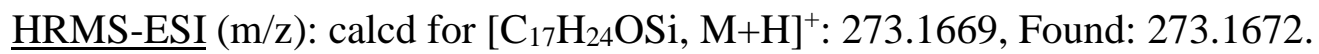

(E)-4-cyclopropyl-2-phenyl-1-(trimethylsilyl)penta-1,4-dien-3-one (1be)<smiles>C=C(C(=O)C1CC1)c1ccccc1</smiles>

Serial number: cj-3-48, Route B (method c) was followed with corresponding ketone S5 (190 mg, $0.7 \mathrm{mmol})$, bis(dimethylamino)methane (755 mg, $7.4 \mathrm{mmol})$, acetic anhydride (755 mg, $7.4 \mathrm{mmol})$ in anhydrous DMF $(1 \mathrm{~mL})$ to afford $\mathbf{1 b e}$ as yellow oil (176 mg, 88\% yield for the last step). TLC $R_{f}=0.72(\mathrm{PE} / \mathrm{EA}=10: 1, \mathrm{v} / \mathrm{v})$. 
$\underline{{ }^{1} \mathrm{H} N M R}\left(400 \mathrm{MHz}, \mathrm{CDCl}_{3}\right) \delta 7.36-7.29(\mathrm{~m}, 3 \mathrm{H}, \mathrm{Ar}-\mathrm{H}), 7.27-7.23(\mathrm{~m}, 2 \mathrm{H}, \mathrm{Ar}-\mathrm{H})$, $6.36(\mathrm{~s}, 1 \mathrm{H},=$ C$\underline{\mathrm{HTMS}}), 5.67\left(\mathrm{~s}, 1 \mathrm{H},=\mathrm{C}_{2}\right), 5.43\left(\mathrm{~s}, 1 \mathrm{H},=\underline{\mathrm{C}}_{2}\right), 1.80(\mathrm{~m}$, $\left.1 \mathrm{H}, \mathrm{C} \underline{\mathrm{H}}\left(\mathrm{CH}_{2}\right)_{2}\right), 0.86-0.74\left(\mathrm{~m}, 2 \mathrm{H}, \mathrm{CH}\left(\mathrm{C}_{2}\right)_{2}\right), 0.56-0.46(\mathrm{~m}, 2 \mathrm{H}$, $\left.\mathrm{CH}\left(\underline{\mathrm{C}}_{2}\right)_{2}\right),-0.07$ (s, 9H, $\left.\mathrm{SiMe}_{3}\right)$.

${ }^{13} \mathrm{C}$ NMR $\left(101 \mathrm{MHz}, \mathrm{CDCl}_{3}\right) \delta 199.4,156.0,150.0,140.7,138.9,129.2,128.2,128.2$, $121.2,12.3,8.0,0.0$.

HRMS-ESI (m/z): calcd for [ $\left.\mathrm{C}_{17} \mathrm{H}_{22} \mathrm{OSi}, \mathrm{M}+\mathrm{Na}\right]^{+}:$293.1332, Found: 293.1337.

\section{(E)-7-methyl-4-methylene-2-phenyl-1-(trimethylsilyl)octa-1,7-dien-3-one (1bf)}<smiles>C=CCCC(=C)C(=O)/C(=C/C)c1ccccc1</smiles>

$1 \mathrm{bf}$

Serial number: cj-3-34, Route B (method c) was followed with corresponding ketone S5 (533 mg, $2.0 \mathrm{mmol})$, bis(dimethylamino)methane (2.0 g, $19.6 \mathrm{mmol})$, acetic anhydride (2.0 g, $19.6 \mathrm{mmol})$ in anhydrous DMF (2 mL) to afford $\mathbf{1 b f}$ as colorless oil (423 mg, 76\% yield for the last step). TLC $R_{f}=0.82(\mathrm{PE} / \mathrm{EA}=10: 1, \mathrm{v} / \mathrm{v})$.

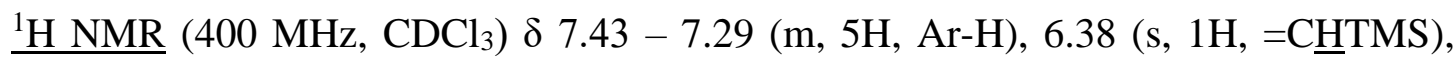
$5.97\left(\mathrm{~s}, 1 \mathrm{H},=\underline{\mathrm{C}}_{2}\right), 5.93-5.80\left(\mathrm{~m}, 2 \mathrm{H},=\underline{\mathrm{C}}_{2}, \mathrm{C} \underline{\mathrm{H}}=\mathrm{CH}_{2}\right), 5.06(\mathrm{dd}, J=17.1$, $\left.13.7 \mathrm{~Hz}, 2 \mathrm{H}, \mathrm{CH}=\underline{\mathrm{C}}_{2}\right), 2.52\left(\mathrm{t}, J=7.6 \mathrm{~Hz}, 2 \mathrm{H}, \underline{\mathrm{C}}_{2} \mathrm{CH}_{2} \mathrm{CH}=\mathrm{CH}_{2}\right), 2.29$ (q, $\left.J=6.8 \mathrm{~Hz}, 2 \mathrm{H}, \mathrm{CH}_{2} \underline{\mathrm{H}}_{2} \mathrm{CH}=\mathrm{CH}_{2}\right), 0.00$ (s, 9H, SiMe $\left.\underline{\mathrm{M}}_{3}\right)$.

${ }^{13} \mathrm{C} \mathrm{NMR}\left(101 \mathrm{MHz}, \mathrm{CDCl}_{3}\right) \delta 199.3,155.9,147.5,140.0,138.9,138.1,129.0,128.2$, $128.2,126.9,115.5,32.6,31.2,0.0$.

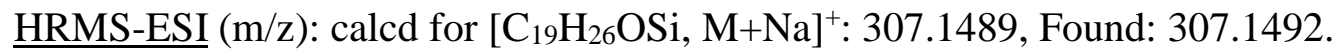




\section{(E)-6-chloro-4-methylene-2-phenyl-1-(trimethylsilyl)hex-1-en-3-one (1bg)}<smiles>C=C(CCCl)C(=O)C(=C[13CH3])c1ccccc1</smiles>

Serial number: cj-3-67, Route A was followed with corresponding dienol S4 (1.1 g, $3.7 \mathrm{mmol})$, DMP (2.3 g, $5.5 \mathrm{mmol})$ in DCM (40 mL) to afford $\mathbf{1 b g}$ as colorless oil (976 mg, 91\% yield for the last step). TLC $R_{f}=0.74(\mathrm{PE} / \mathrm{EA}=10: 1, \mathrm{v} / \mathrm{v})$.

$\underline{{ }^{1} \mathrm{H} \mathrm{NMR}}\left(400 \mathrm{MHz}, \mathrm{CDCl}_{3}\right) \delta 7.34-7.31(\mathrm{~m}, 3 \mathrm{H}, \mathrm{Ar}-\mathrm{H}), 7.26-7.23$ (m, 2H, Ar-H), $6.36(\mathrm{~s}, 1 \mathrm{H},=\mathrm{C} \underline{\mathrm{HTMS}}), 6.06\left(\mathrm{~d}, J=0.8 \mathrm{~Hz}, 1 \mathrm{H},=\underline{\mathrm{CH}}_{2}\right), 5.96(\mathrm{~d}, J=1.0 \mathrm{~Hz}$, $\left.1 \mathrm{H},=\underline{\mathrm{C}}_{2}\right), 3.66\left(\mathrm{t}, J=6.6 \mathrm{~Hz}, 2 \mathrm{H}, \mathrm{CH}_{2} \underline{\mathrm{CH}}_{2} \mathrm{Cl}\right), 2.81(\mathrm{td}, J=6.6,1.1 \mathrm{~Hz}$, $2 \mathrm{H}, \mathrm{C}_{2} \mathrm{CH}_{2} \mathrm{Cl}$ ), -0.07 (s, 9H, $\left.\underline{\mathrm{Sie}}_{3}\right)$.

${ }^{13} \mathrm{C}$ NMR $\left(101 \mathrm{MHz}, \mathrm{CDCl}_{3}\right) \delta 198.7,155.4,144.0,140.8,138.7,130.1,129.1,128.3$, $128.3,43.3,35.4,0.0$.

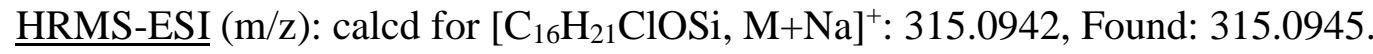

\section{(E)-6-methoxy-4-methylene-2-phenyl-1-(trimethylsilyl)hex-1-en-3-one (1bh)}<smiles>C=C(CCOC)C(=O)C(=O)c1ccccc1</smiles>

Serial number: cj-3-66, Route A was followed with corresponding dienol S4 (894 mg, $3.1 \mathrm{mmol})$, DMP (2.0 g, $4.6 \mathrm{mmol})$ in DCM $(30 \mathrm{~mL})$ to afford $\mathbf{1 b h}$ as colorless oil (778 mg, 87\% yield for the last step). TLC $R_{f}=0.49(\mathrm{PE} / \mathrm{EA}=10: 1, \mathrm{v} / \mathrm{v})$.

${ }^{1} \mathrm{H}$ NMR $\left(400 \mathrm{MHz}, \mathrm{CDCl}_{3}\right) \delta 7.28-7.22(\mathrm{~m}, 3 \mathrm{H}, \mathrm{Ar}-\mathrm{H}), 7.21-7.15$ (m, 2H, Ar-H), $6.26(\mathrm{~s}, 1 \mathrm{H},=\mathrm{C} \underline{\mathrm{HTMS}}), 5.87\left(\mathrm{~s}, 1 \mathrm{H},=\mathrm{C}_{2}\right), 5.81\left(\mathrm{~s}, 1 \mathrm{H},=\mathrm{C}_{2}\right), 3.42(\mathrm{t}, J=$ $\left.6.5 \mathrm{~Hz}, 2 \mathrm{H}, \mathrm{CH}_{2} \mathrm{C}_{2} \mathrm{OMe}\right), 3.24(\mathrm{~s}, 3 \mathrm{H}, \mathrm{OMe}), 2.55(\mathrm{t}, J=6.5 \mathrm{~Hz}, 2 \mathrm{H}$, $\left.\underline{\mathrm{C}}_{2} \mathrm{CH}_{2} \mathrm{OMe}\right),-0.15$ (s, 9H, SiMe 3 ).

${ }^{13} \mathrm{C} \mathrm{NMR}\left(101 \mathrm{MHz}, \mathrm{CDCl}_{3}\right) \delta 199.2,155.7,145.3,140.4,138.9,129.1,128.2,128.2$, $128.1,71.3,58.8,32.4,0.0$.

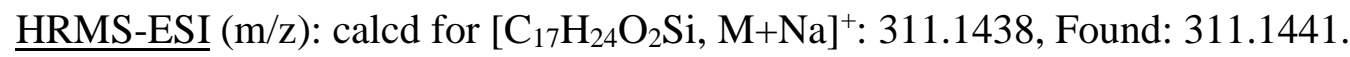




\section{(E)-4-benzyl-2-phenyl-1-(trimethylsilyl)penta-1,4-dien-3-one (1bi)}<smiles>C=C(Cc1ccccc1)C(=O)C(=O)c1ccccc1</smiles>

Serial number: cj-3-73, Route A was followed with corresponding dienol S4 (1.3 g, $4.0 \mathrm{mmol})$, DMP (2.0 g, $4.8 \mathrm{mmol})$ in DCM (30 mL) to afford $\mathbf{1 b i}$ as colorless oil (1.1 $\mathrm{g}, 84 \%$ yield for the last step), TLC $R_{f}=0.76(\mathrm{PE} / \mathrm{EA}=10: 1, \mathrm{v} / \mathrm{v})$.

${ }^{1} \mathrm{H}$ NMR $\left(400 \mathrm{MHz}, \mathrm{CDCl}_{3}\right) \delta 7.32-7.27$ (m, 5H, Ar-H), $7.24-7.17$ (m, 5H, Ar-H), $6.28(\mathrm{~s}, 1 \mathrm{H},=\mathrm{C} \underline{\mathrm{HT} M S}), 5.93\left(\mathrm{~d}, J=1.0 \mathrm{~Hz}, 1 \mathrm{H},=\underline{\mathrm{C}}_{2}\right), 5.66\left(\mathrm{~m}, 1 \mathrm{H},=\underline{\mathrm{C}}_{2}\right)$, 3.67 (s, 2H, $\left.\underline{\mathrm{H}}_{2} \mathrm{Ph}\right),-0.09$ (s, 9H, $\left.\mathrm{SiMe}_{3}\right)$.

${ }^{13} \mathrm{C} \mathrm{NMR}\left(101 \mathrm{MHz}, \mathrm{CDCl}_{3}\right) \delta 198.8,155.7,147.8,140.4,139.1,138.9,129.6,129.1$, $128.8,128.3,128.2,127.6,126.6,38.2,0.0$.

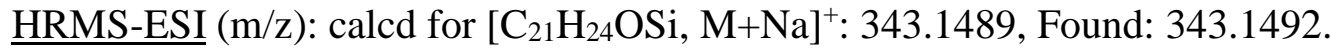

\section{(E)-2,4-diphenyl-1-(trimethylsilyl)penta-1,4-dien-3-one (1ca)}<smiles>C=C(C(=O)C(=C)c1ccccc1)c1ccccc1</smiles>

Serial number: cj-3-12, Route B (method a) was followed to afford 1ca as yellow oil (643 mg, 94\% yield for the last step). TLC $R_{f}=0.71(\mathrm{PE} / \mathrm{EA}=10: 1, \mathrm{v} / \mathrm{v})$.

${ }^{1} \mathrm{H} \mathrm{NMR}\left(400 \mathrm{MHz}, \mathrm{CDCl}_{3}\right) \delta 7.36-7.28(\mathrm{~m}, 8 \mathrm{H}, \mathrm{Ar}-\mathrm{H}), 7.21-7.14$ (m, 2H, Ar-H), $6.78(\mathrm{~s}, 1 \mathrm{H},=\mathrm{CH}$ TMS $), 5.93\left(\mathrm{~s}, 1 \mathrm{H},=\underline{\mathrm{CH}}_{2}\right), 5.80\left(\mathrm{~s}, 1 \mathrm{H},=\underline{\mathrm{C}}_{2}\right),-0.13(\mathrm{~s}$, 9H, $\left.\mathrm{SiMe}_{3}\right)$.

${ }^{13} \mathrm{C} \mathrm{NMR}\left(101 \mathrm{MHz}, \mathrm{CDCl}_{3}\right) \delta 198.7,156.0,149.1,145.7,138.8,137.9,129.8,129.0$, $128.8,128.4,127.8,122.5,0.0$.

$\underline{\text { HRMS-ESI }}(\mathrm{m} / \mathrm{z})$ : calcd for $\left[\mathrm{C}_{20} \mathrm{H}_{22} \mathrm{OSi}, \mathrm{M}+\mathrm{Na}\right]^{+}:$329.1332, Found: 329.1336. 
<smiles>C=C(C(=O)C(=C[S+](C)(=O)=O)c1ccccc1C)c1ccccc1</smiles>

Serial number: cj-3-111, Route B (method a) was followed to afford $\mathbf{1 c b}$ as yellow oil (416 mg, 77\% yield for the last step). TLC $R_{f}=0.75(\mathrm{PE} / \mathrm{EA}=10: 1, \mathrm{v} / \mathrm{v})$.

${ }^{1} \mathrm{H}$ NMR $\left(400 \mathrm{MHz}, \mathrm{CDCl}_{3}\right) \delta 7.27(\mathrm{~m}, 3 \mathrm{H}, \mathrm{Ar}-\mathrm{H}), 7.19(\mathrm{~d}, J=7.2 \mathrm{~Hz}, 1 \mathrm{H}, \mathrm{Ar}-\mathrm{H})$, 7.12 (m, 4H, Ar-H), 6.99 (d, J=7.5 Hz, 1H, Ar-H), 6.57 (s, 1H, =CㅁTMS), $6.21\left(\mathrm{~s}, 1 \mathrm{H},=\underline{\mathrm{C}}_{2}\right), 5.81\left(\mathrm{~s}, 1 \mathrm{H},=\underline{\mathrm{C}}_{2}\right), 2.13(\mathrm{~s}, 3 \mathrm{H}, \mathrm{Me}),-0.13(\mathrm{~s}, 9 \mathrm{H}$, $\left.\mathrm{SiMe}_{3}\right)$.

${ }^{13} \mathrm{C}$ NMR $\left(101 \mathrm{MHz}, \mathrm{CDCl}_{3}\right) \delta 197.6,155.8,149.6,142.0,138.7,138.3,138.2,136.2$, $130.5,130.1,129.4,128.5,128.29,128.27,126.2,20.6,0.0$.

HRMS-ESI (m/z): calcd for [ $\left.\mathrm{C}_{21} \mathrm{H}_{24} \mathrm{OSi}, \mathrm{M}+\mathrm{H}\right]^{+}: 321.1669$, Found: 321.1672.

\section{(E)-2-phenyl-4-(thiophen-3-yl)-1-(trimethylsilyl)penta-1,4-dien-3-one (1cc)}<smiles>C=C(C(=O)C(=CC(C)(C)C)c1ccccc1)c1ccsc1</smiles>

Serial number: cj-3-25, Route B (method b) was followed to afford 1cc as yellow oil (527 mg, 90\% yield for the last step). TLC $R_{f}=0.64(\mathrm{PE} / \mathrm{EA}=10: 1, \mathrm{v} / \mathrm{v})$.

$\underline{{ }^{1} \mathrm{H} \mathrm{NMR}}\left(400 \mathrm{MHz}, \mathrm{CDCl}_{3}\right) \delta 7.38-7.27$ (m, 5H, Ar-H), 7.23 (m, 3H, Ar-H), 6.77 (s, $1 \mathrm{H},=\mathrm{C} \underline{\mathrm{HTMS}}), 6.02\left(\mathrm{~s}, 1 \mathrm{H},=\underline{\mathrm{C}}_{2}\right), 5.75\left(\mathrm{~s}, 1 \mathrm{H},=\underline{\mathrm{C}}_{2}\right),-0.09(\mathrm{~s}, 9 \mathrm{H}$, $\left.\mathrm{SiMe}_{3}\right)$.

${ }^{13} \mathrm{C} \mathrm{NMR}\left(101 \mathrm{MHz}, \mathrm{CDCl}_{3}\right) \delta 198.3,155.9,145.4,143.0,138.8,137.9,129.6,128.4$, 128.4, 126.8, 126.1, 124.1, 121.9, 0.0.

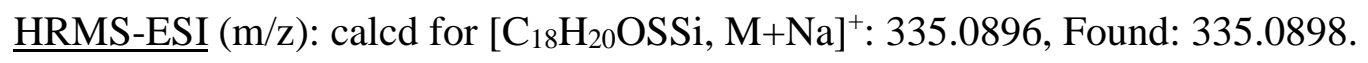




\section{(E)-2-benzyl-4-methyl-1-(trimethylsilyl)penta-1,4-dien-3-one (1da)}<smiles>C=C(C)C(=O)C(=C[As])Cc1ccccc1</smiles>

Serial number: cj-2-76, Route E was followed with corresponding dienol S13 (300 mg, $1.2 \mathrm{mmol})$, DMP (587 mg, $1.4 \mathrm{mmol})$, pyridine (363 mg, $4.6 \mathrm{mmol})$ in DCM (15 mL) to afford 1da as colorless oil (271 mg, 89\% yield for the last step). TLC $R_{f}=0.74$ $(\mathrm{PE} / \mathrm{EA}=10: 1, \mathrm{v} / \mathrm{v})$.

$\underline{{ }^{1} \mathrm{H} \text { NMR }}\left(400 \mathrm{MHz}, \mathrm{CDCl}_{3}\right) \delta 7.26-7.12(\mathrm{~m}, 5 \mathrm{H}, \mathrm{Ar}-\mathrm{H}), 6.24(\mathrm{~s}, 1 \mathrm{H},=\mathrm{C} \underline{\mathrm{HTMS}})$, $5.63\left(\mathrm{~d}, J=1.5 \mathrm{~Hz}, 1 \mathrm{H},=\mathrm{C}_{2}\right), 5.54\left(\mathrm{~s}, 1 \mathrm{H},=\mathrm{CH}_{2}\right), 3.83\left(\mathrm{~s}, 2 \mathrm{H}, \mathrm{PhC}_{2}\right)$, 1.83 (s, 3H, $\left.\underline{\mathrm{CH}}_{3}\right), 0.24$ (s, 9H, $\left.\mathrm{SiMe}_{3}\right)$.

${ }^{13} \mathrm{C}$ NMR $\left(101 \mathrm{MHz}, \mathrm{CDCl}_{3}\right) \delta 200.1,153.8,143.6,139.6,138.7,128.7,128.4,126.2$, $125.9,37.8,18.3,0.0$.

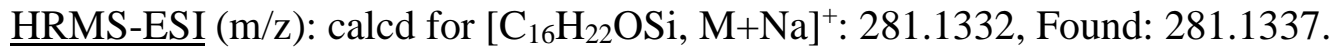

\section{(E)-2-methyl-4-((trimethylsilyl)methylene)oct-1-en-3-one (1db)}<smiles>C=C(C)C(=O)C(=CC(C)(C)C)CCCC</smiles>

Serial number: cj-1-110, Route D was followed with corresponding dienol S10 (260 mg, $1.2 \mathrm{mmol}$ ), DMP (731 mg, $1.7 \mathrm{mmol})$, pyridine (363 mg, $4.6 \mathrm{mmol})$ in DCM (15 $\mathrm{mL})$ to afford $\mathbf{1 d b}$ as colorless oil $\left(230 \mathrm{mg}, 88 \%\right.$ yield for the last step). TLC $R_{f}=$ $0.83(\mathrm{PE} / \mathrm{EA}=10: 1, \mathrm{v} / \mathrm{v})$.

$\underline{{ }^{1} \mathrm{H} \text { NMR }}\left(400 \mathrm{MHz}, \mathrm{CDCl}_{3}\right) \delta 6.01(\mathrm{~s}, 1 \mathrm{H},=\mathrm{C} \underline{\mathrm{HTMS}}), 5.72\left(\mathrm{~m}, 1 \mathrm{H},=\underline{\mathrm{C}}_{2}\right), 5.64(\mathrm{~d}, J$ $\left.=1.5 \mathrm{~Hz}, 1 \mathrm{H},=\underline{\mathrm{C}}_{2}\right), 2.43\left(\mathrm{dd}, J=9.0,5.9 \mathrm{~Hz}, 2 \mathrm{H}, \underline{\mathrm{C}}_{2}\left(\mathrm{CH}_{2}\right)_{2} \mathrm{CH}_{3}\right), 1.93$ $\left(\mathrm{d}, J=1.3 \mathrm{~Hz}, 3 \mathrm{H}, \underline{\mathrm{C}}_{3}\right), 1.31\left(\mathrm{~m} 4 \mathrm{H}, \mathrm{CH}_{2}\left(\underline{\mathrm{C}}_{2}\right)_{2} \mathrm{CH}_{3}\right), 0.88$ (t, $J=6.7 \mathrm{~Hz}$, $\left.3 \mathrm{H}, \mathrm{CH}_{2}\left(\mathrm{CH}_{2}\right)_{2} \underline{\mathrm{H}}_{3}\right), 0.17$ (s, 9H, SiMe 3 ).

${ }^{13} \mathrm{C} \mathrm{NMR}\left(101 \mathrm{MHz}, \mathrm{CDCl}_{3}\right) \delta 201.2,156.3,144.1,137.6,126.0,32.4,31.5,23.0$, $18.4,14.1,0.0$. 


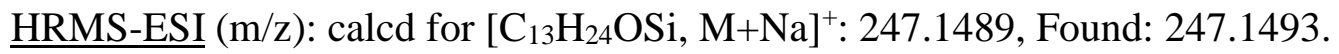

(E)-2,5-dimethyl-4-((trimethylsilyl)methylene)hex-1-en-3-one (1dc)<smiles>C=C(C)C(=O)C(=C[As])C(C)C</smiles>

Serial number: cj-2-191, Route D was followed with corresponding dienol S10 (577 mg, $2.7 \mathrm{mmol})$, DMP (1.4 g, $3.3 \mathrm{mmol})$, pyridine (860 mg, $10.9 \mathrm{mmol})$ in DCM (20 $\mathrm{mL}$ ) to afford 1dc as yellow oil (440 $\mathrm{mg}, 77 \%$ yield for the last step). TLC $R_{f}=0.78$ $(\mathrm{PE} / \mathrm{EA}=10: 1, \mathrm{v} / \mathrm{v})$.

${ }^{1} \mathrm{H}$ NMR $\left(400 \mathrm{MHz}, \mathrm{CDCl}_{3}\right) \delta 5.84-5.79(\mathrm{~m}, 1 \mathrm{H},=\mathrm{C} \underline{\mathrm{HTMS}}), 5.78-5.73(\mathrm{~m}, 1 \mathrm{H}$, $\left.=\underline{\mathrm{C}}_{2}\right), 5.62\left(\mathrm{~s}, 1 \mathrm{H},=\underline{\mathrm{CH}}_{2}\right), 2.77$ (hept, $\left.J=6.9 \mathrm{~Hz}, 1 \mathrm{H}, \underline{\mathrm{CMMe}}_{2}\right), 1.94-1.85$ (m, 3H, $\left.\underline{\mathrm{H}}_{3}\right), 1.14$ (d, J=6.9 Hz, 6H, $\left.\mathrm{CHMe} 2\right), 0.17$ (s, 9H, SiMe 3 ).

$\underline{{ }^{13} \mathrm{C} \mathrm{NMR}}\left(101 \mathrm{MHz}, \mathrm{CDCl}_{3}\right) \delta 201.5,160.6,144.9,133.2,127.9,34.4,21.8,17.5$, 0.0 .

HRMS-ESI $(\mathrm{m} / \mathrm{z})$ : calcd for $\left[\mathrm{C}_{12} \mathrm{H}_{22} \mathrm{OSi}, \mathrm{M}+\mathrm{H}\right]^{+}:$211.1513, Found: 211.1516.

\section{(E)-2-cyclopentyl-4-methyl-1-(trimethylsilyl)penta-1,4-dien-3-one (1dd)}<smiles>C=C(C)C(=O)/C(=C/C)C1CCCC1</smiles>

Serial number: cj-2-35, Route D was followed with corresponding dienol S10 (357 mg, $1.5 \mathrm{mmol}$ ), DMP (763 mg, $1.8 \mathrm{mmol})$, pyridine (474 mg, $6.0 \mathrm{mmol})$ in DCM (25 $\mathrm{mL})$ to afford 1dd as colorless oil (150 mg, $40 \%$ yield for the last step). TLC $R_{f}=$ $0.86(\mathrm{PE} / \mathrm{EA}=10: 1, \mathrm{v} / \mathrm{v})$.

${ }^{1} \mathrm{H}$ NMR $\left(400 \mathrm{MHz}, \mathrm{CDCl}_{3}\right) \delta 5.84-5.79(\mathrm{~m}, 1 \mathrm{H},=\mathrm{C} \underline{\mathrm{HTMS}}), 5.77\left(\mathrm{~s}, 1 \mathrm{H},=\mathrm{C}_{2}\right)$, $5.65\left(\mathrm{~s}, 1 \mathrm{H},=\underline{\mathrm{C}}_{2}\right), 2.86-2.75\left(\mathrm{~m}, 1 \mathrm{H}, \mathrm{C} \underline{\mathrm{H}}\left(\mathrm{CH}_{2}\right)_{4}\right), 1.95-1.85(\mathrm{~m}, 3 \mathrm{H}$, $\left.\mathrm{C}_{3}\right), 1.78-1.62\left(\mathrm{~m}, 6 \mathrm{H}, \mathrm{CH}\left(\underline{\mathrm{CH}}_{2}\right)_{4}\right), 1.57-1.48\left(\mathrm{~m}, 2 \mathrm{H}, \mathrm{CH}\left(\mathrm{C}_{2}\right)_{4}\right), 0.17$ (s, 9H, $\left.\mathrm{SiMe}_{3}\right)$. 
${ }^{{ }^{13} \mathrm{C} \mathrm{NMR}}\left(101 \mathrm{MHz}, \mathrm{CDCl}_{3}\right) \delta 201.7,158.5,144.9,133.8,127.9,45.5,32.1,25.6$, $17.3,0.0$.

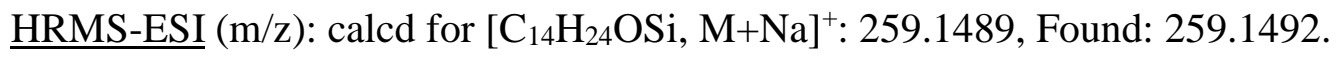

\section{(E)-2-cyclohexyl-4-methyl-1-(trimethylsilyl)penta-1,4-dien-3-one (1de)}<smiles>C=C(C)C(=O)C(=CC(C)(C)C)C1CCCCC1</smiles>

Serial number: cj-1-115, Route D was followed with corresponding dienol S10 (320 mg, $1.3 \mathrm{mmol}$ ), DMP (646 mg, $1.5 \mathrm{mmol}$ ), pyridine (400 mg, $5.0 \mathrm{mmol})$ in DCM (15 $\mathrm{mL}$ ) to afford 1de as colorless oil ( $280 \mathrm{mg}, 87 \%$ yield for the last step). TLC $R_{f}=0.88$ $(\mathrm{PE} / \mathrm{EA}=10: 1, \mathrm{v} / \mathrm{v})$.

$\underline{{ }^{1} \mathrm{H} \mathrm{NMR}}\left(400 \mathrm{MHz}, \mathrm{CDCl}_{3}\right) \delta 5.82(\mathrm{~m}, 1 \mathrm{H},=\mathrm{C} \underline{\mathrm{HTMS}}), 5.79\left(\mathrm{~m}, 1 \mathrm{H},=\underline{\mathrm{C}}_{2}\right), 5.55(\mathrm{~s}$, $\left.1 \mathrm{H},=\underline{\mathrm{C}}_{2}\right), 2.41\left(\mathrm{tt}, J=12.0,3.3 \mathrm{~Hz}, 1 \mathrm{H}, \mathrm{C} \underline{\mathrm{H}}\left(\mathrm{CH}_{2}\right)_{5}\right), 1.89(\mathrm{t}, J=1.2 \mathrm{~Hz}$, $\left.3 \mathrm{H}, \mathrm{C}_{3}\right), 1.78-1.71\left(\mathrm{~m}, 2 \mathrm{H}, \mathrm{CH}\left(\mathrm{CH}_{2}\right)_{5}\right), 1.64(\mathrm{~d}, J=3.5 \mathrm{~Hz}, 3 \mathrm{H}$, $\left.\mathrm{CH}\left(\mathrm{C}_{2}\right)_{5}\right), 1.54-1.41\left(\mathrm{~m}, 2 \mathrm{H}, \mathrm{CH}\left(\mathrm{C}_{2}\right)_{5}\right), 1.29-1.11\left(\mathrm{~m}, 3 \mathrm{H}, \mathrm{CH}\left(\underline{\mathrm{CH}}_{2}\right)_{5}\right)$, 0.17 (s, 9H, $\left.\mathrm{SiMe}_{3}\right)$.

$\underline{{ }^{13} \mathrm{C} \mathrm{NMR}}\left(101 \mathrm{MHz}, \mathrm{CDCl}_{3}\right) \delta 201.6,160.4,144.8,132.3,128.4,45.0,31.8,26.5$, $25.6,17.3,0.0$.

HRMS-ESI (m/z): calcd for [ $\left.\mathrm{C}_{15} \mathrm{H}_{26} \mathrm{OSi}, \mathrm{M}+\mathrm{H}\right]^{+}:$251.1826, Found: 251.1828.

(E)-2-(cyclohex-1-en-1-yl)-4-methyl-1-(trimethylsilyl)penta-1,4-dien-3-one (1ea)<smiles>C=C(C)C(=O)C(=CC(C)C)C1=CCCCC1</smiles>

Serial number: cj-2-66, Route C was followed with corresponding dienol S8 (400 mg, $1.6 \mathrm{mmol}), \mathrm{DMP}$ (814 mg, $1.9 \mathrm{mmol})$, pyridine (505 mg, $6.4 \mathrm{mmol})$ in DCM (20 mL) to afford 1ea as yellow oil (344 mg, 86\% yield for the last step). TLC $R_{f}=0.85$ $(\mathrm{PE} / \mathrm{EA}=10: 1, \mathrm{v} / \mathrm{v})$. 
$\underline{{ }^{1} \mathrm{H} \text { NMR }}\left(400 \mathrm{MHz}, \mathrm{CDCl}_{3}\right) \delta 5.87(\mathrm{~s}, 1 \mathrm{H},=\mathrm{C} \underline{\mathrm{HTMS}}), 5.78\left(\mathrm{~m}, 2 \mathrm{H},=\underline{\mathrm{CH}}_{2}\right), 5.66(\mathrm{~m}$, $\left.1 \mathrm{H},=\mathrm{C}_{\underline{H}} \mathrm{H}_{2}\right), 2.10\left(\mathrm{td}, J=5.7,3.0 \mathrm{~Hz}, 2 \mathrm{H},=\mathrm{CHC}_{2}\right), 2.00(\mathrm{~m}, 2 \mathrm{H}$, $\left.=\mathrm{CHCH}_{2}\left(\mathrm{CH}_{2}\right)_{2} \mathrm{CH}_{2}\right), 1.91\left(\mathrm{t}, J=1.1 \mathrm{~Hz}, 3 \mathrm{H}, \underline{\mathrm{C}}_{3}\right), 1.61(\mathrm{~m}, 4 \mathrm{H}$, $\left.=\mathrm{CHCH}_{2}\left(\underline{\mathrm{C}}_{2}\right)_{2} \mathrm{CH}_{2}\right), 0.12\left(\mathrm{~s}, 9 \mathrm{H}, \mathrm{SiMe}_{3}\right)$.

${ }^{13} \mathrm{C} \mathrm{NMR}\left(101 \mathrm{MHz}, \mathrm{CDCl}_{3}\right) \delta 199.9,158.3,143.8,137.2,136.2,127.5,126.8,27.9$, $25.1,22.3,21.5,17.8,0.0$.

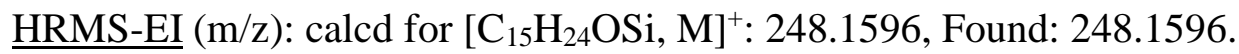

(E)-2-methyl-4-((trimethylsilyl)methylene)dodec-1-en-5-yn-3-one (1fa)<smiles>C=C(C)C(=O)/C(C#CCCCCC)=C\C(=O)O</smiles>

Serial number: cj-2-73, Route F was followed with corresponding dienol S14 (353 mg, $1.3 \mathrm{mmol}), \mathrm{DMP}$ (807 mg, $1.9 \mathrm{mmol})$, pyridine (401 mg, $5.1 \mathrm{mmol})$ in DCM (20 mL) to afford 1fa as yellow oil (321 mg, 91\% yield for the last step). TLC $R_{f}=0.92$ $(\mathrm{PE} / \mathrm{EA}=10: 1, \mathrm{v} / \mathrm{v})$.

$\underline{{ }^{1} \mathrm{H} \mathrm{NMR}}\left(400 \mathrm{MHz}, \mathrm{CDCl}_{3}\right) \delta 6.58(\mathrm{~s}, 1 \mathrm{H},=\mathrm{C} \underline{\mathrm{HTMS}}), 5.91\left(\mathrm{t}, J=1.0 \mathrm{~Hz}, 1 \mathrm{H},=\mathrm{C}_{2}\right)$, $5.83-5.80\left(\mathrm{~m}, 1 \mathrm{H},=\underline{\mathrm{C}}_{2}\right), 2.36\left(\mathrm{t}, J=7.1 \mathrm{~Hz}, 2 \mathrm{H}, \mathrm{C}_{2}\left(\mathrm{CH}_{2}\right)_{4} \mathrm{CH}_{3}\right), 1.93$ $\left(\mathrm{dd}, J=1.5,0.9 \mathrm{~Hz}, 3 \mathrm{H}, \underline{\mathrm{C}}_{3}\right), 1.59-1.51\left(\mathrm{~m}, 2 \mathrm{H}, \mathrm{CH}_{2} \mathrm{C}_{2}\left(\mathrm{CH}_{2}\right)_{3} \mathrm{CH}_{3}\right)$, $1.39\left(\mathrm{~d}, \quad J=1.2 \mathrm{~Hz}, 2 \mathrm{H}, \quad \mathrm{CH}_{2} \mathrm{CH}_{2} \mathrm{CH}_{2}\left(\mathrm{CH}_{2}\right)_{2} \mathrm{CH}_{3}\right), 1.29(\mathrm{~m}, 4 \mathrm{H}$, $\left.\mathrm{CH}_{2} \mathrm{CH}_{2} \mathrm{CH}_{2}\left(\mathrm{C}_{2}\right)_{2} \mathrm{CH}_{3}\right), 0.88\left(\mathrm{t}, J=6.9 \mathrm{~Hz}, 3 \mathrm{H},\left(\mathrm{CH}_{2}\right)_{5} \mathrm{C}_{3}\right), 0.22(\mathrm{~s}, 9 \mathrm{H}$, $\left.\mathrm{SiMe}_{3}\right)$.

${ }^{13} \mathrm{C} \mathrm{NMR}\left(101 \mathrm{MHz}, \mathrm{CDCl}_{3}\right) \delta 195.8,147.1,142.3,137.6,127.2,98.8,78.4,31.4$, 28.7, 28.3, 22.6, 19.7, 18.2, 14.1, -1.3.

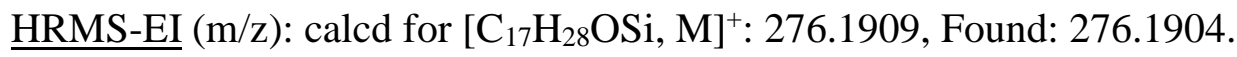


(1E,4E)-4-methyl-2-phenyl-1-(trimethylsilyl)hexa-1,4-dien-3-one (1ga)<smiles>C/C=C(\C)C(=O)/C(=C/CS(C)(=O)=O)c1ccccc1</smiles>

Serial number: cj-2-63, Route A was followed with corresponding dienol S4 (310 mg, $1.2 \mathrm{mmol}$ ), DMP (606 mg, $1.4 \mathrm{mmol})$, pyridine (376 mg, $4.8 \mathrm{mmol})$ in DCM (20 mL) to afford 1ga as a yellow oil (267 $\mathrm{mg}, 86 \%$ yield for the last step). TLC $R_{f}=0.83$ $(\mathrm{PE} / \mathrm{EA}=10: 1, \mathrm{v} / \mathrm{v})$.

매 NMR (400 MHz, $\left.\mathrm{CDCl}_{3}\right) \delta 7.30(\mathrm{~s}, 5 \mathrm{H}, \mathrm{Ar}-\mathrm{H}), 6.76(\mathrm{~d}, J=7.1 \mathrm{~Hz}, 1 \mathrm{H},=\mathrm{C} \underline{\mathrm{HMe}})$, $6.00(\mathrm{~s}, 1 \mathrm{H},=$ C $\underline{\mathrm{HTMS}}), 1.86(\mathrm{~d}, J=7.0 \mathrm{~Hz}, 3 \mathrm{H},=\mathrm{CHMe}), 1.82(\mathrm{~s}, 3 \mathrm{H}$, $\left.\mathrm{C}_{3}\right),-0.07$ (s, 9H, $\left.\mathrm{SiMe}_{3}\right)$.

${ }^{13} \mathrm{C} \mathrm{NMR}\left(101 \mathrm{MHz}, \mathrm{CDCl}_{3}\right) \delta 200.0,156.3,142.1,139.1,137.4,136.3,128.6,128.1$, 128.0, 15.1, 11.6, 0.0.

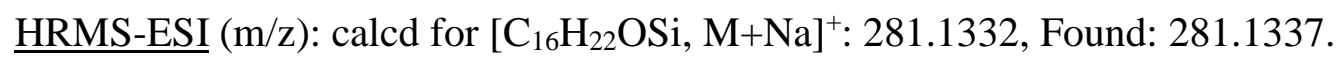

(1E,4E)-4-isopropyl-2-phenyl-1-(trimethylsilyl)hexa-1,4-dien-3-one (1gb)<smiles>C/C=C(\C(=O)/C(=C/C)c1ccccc1)C(C)C</smiles>

Serial number: cj-4-123, Route A was followed with corresponding dienol S4 (240 mg, $0.8 \mathrm{mmol})$, DMP (530 mg, $1.2 \mathrm{mmol})$, pyridine (262 mg, $3.3 \mathrm{mmol})$ in DCM (10 $\mathrm{mL}$ ) to afford $\mathbf{1 g b}$ as a colorless oil (209 $\mathrm{mg}, 88 \%$ yield for the last step). TLC $R_{f}=$ $0.85(\mathrm{PE} / \mathrm{EA}=10: 1, \mathrm{v} / \mathrm{v})$.

$\underline{{ }^{1} \mathrm{H} \mathrm{NMR}}\left(400 \mathrm{MHz}, \mathrm{CDCl}_{3}\right) \delta 7.33-7.25(\mathrm{~m}, 3 \mathrm{H}, \mathrm{Ar}-\mathrm{H}), 7.24-7.15(\mathrm{~m}, 2 \mathrm{H}, \mathrm{Ar}-\mathrm{H})$, $6.32(\mathrm{q}, J=7.0 \mathrm{~Hz}, 1 \mathrm{H},=\mathrm{C} \underline{\mathrm{HMe}}), 6.19(\mathrm{~s}, 1 \mathrm{H},=\mathrm{C} \underline{\mathrm{HTMS}}), 2.93-2.67$ (m, $\left.1 \mathrm{H}, \underline{\mathrm{CHMe}}_{2}\right), 1.81(\mathrm{~d}, J=7.1 \mathrm{~Hz}, 3 \mathrm{H},=\mathrm{CHMe}), 1.14(\mathrm{~d}, J=7.0 \mathrm{~Hz}, 6 \mathrm{H}$, $\left.\mathrm{CHMe}_{2}\right),-0.12$ (s, 9H, $\left.\underline{\mathrm{SiMe}}_{3}\right)$.

${ }^{13} \mathrm{C} \mathrm{NMR}\left(101 \mathrm{MHz}, \mathrm{CDCl}_{3}\right) \delta 200.4,157.6,146.6,139.3,139.3,137.8,129.0,128.1$, $127.9,27.9,21.0,14.3,0.0$. 


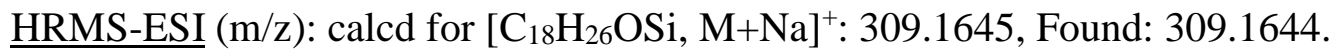

\section{(1E,4E)-2-methyl-1,4-diphenyl-5-(trimethylsilyl)penta-1,4-dien-3-one (1gc)}<smiles>CC(=Cc1ccccc1)C(=O)C(=Cc1ccccc1)c1ccccc1</smiles>

Serial number: cj-4-124, Route A was followed with corresponding dienol S4 (489 mg, $1.5 \mathrm{mmol}$ ), DMP (967 mg, $2.3 \mathrm{mmol}$ ), pyridine (480 mg, $6.1 \mathrm{mmol})$ in DCM (20 $\mathrm{mL}$ ) to afford $1 \mathrm{gc}$ as a colorless oil (365 $\mathrm{mg}, 76 \%$ yield for the last step). TLC $R_{f}=$ $0.81(\mathrm{PE} / \mathrm{EA}=10: 1, \mathrm{v} / \mathrm{v})$.

${ }^{1} \mathrm{H}$ NMR $\left(400 \mathrm{MHz}, \mathrm{CDCl}_{3}\right) \delta 7.53(\mathrm{~s}, 1 \mathrm{H},=\mathrm{CHPh}), 7.42-7.38(\mathrm{~m}, 4 \mathrm{H}, \mathrm{Ar}-\mathrm{H}), 7.37$ - $7.32(\mathrm{~m}, 6 \mathrm{H}$, Ar-H), $6.22(\mathrm{~s}, 1 \mathrm{H},=\mathrm{C} \underline{\mathrm{HTMS}}), 2.13(\mathrm{~s}, 3 \mathrm{H}, \underline{\mathrm{Me}}),-0.03(\mathrm{~s}$, 9H, $\left.\mathrm{SiMe}_{3}\right)$.

$\underline{{ }^{13} \mathrm{C} \text { NMR }}\left(101 \mathrm{MHz}, \mathrm{CDCl}_{3}\right) \delta 200.6,156.1,142.8,139.1,137.8,136.6,136.0,130.0$, $128.8,128.8,128.7,128.2,128.1,13.8,0.0$.

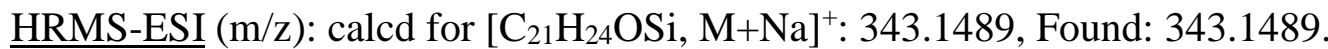

\section{(E)-4-methyl-1,2-diphenyl-1-(trimethylsilyl)penta-1,4-dien-3-one (1ha)}<smiles>C=C(C)C(=O)C(=C(C)Sc1ccccc1)c1ccccc1</smiles>

Serial number: cj-2-13, Route $\mathrm{G}$ was followed with corresponding dienol S18 (418 mg, $1.3 \mathrm{mmol})$, DMP (660 mg, $1.6 \mathrm{mmol})$, pyridine (410 mg, $5.2 \mathrm{mmol})$ in DCM (15 $\mathrm{mL}$ ) to afford 1 ha as white solid (370 $\mathrm{mg}, 89 \%$ yield for the last step). mp: $95-96{ }^{\circ} \mathrm{C}$. TLC $R_{f}=0.76(\mathrm{PE} / \mathrm{EA}=10: 1, \mathrm{v} / \mathrm{v})$.

${ }^{1} \mathrm{H} \mathrm{NMR}\left(400 \mathrm{MHz}, \mathrm{CDCl}_{3}\right) \delta 7.49(\mathrm{~m}, 2 \mathrm{H}, \mathrm{Ar}-\mathrm{H}), 7.39-7.32(\mathrm{~m}, 3 \mathrm{H}, \mathrm{Ar}-\mathrm{H}), 7.22(\mathrm{t}$, $J=7.6 \mathrm{~Hz}, 2 \mathrm{H}, \mathrm{Ar}-\mathrm{H}), 7.16-7.10(\mathrm{~m}, 1 \mathrm{H}, \mathrm{Ar}-\mathrm{H}), 7.02-6.96(\mathrm{~m}, 2 \mathrm{H}$, Ar-H), $5.98-5.95\left(\mathrm{~m}, 1 \mathrm{H},=\mathrm{C}_{2}\right), 5.66-5.63\left(\mathrm{~m}, 1 \mathrm{H},=\underline{\mathrm{CH}}_{2}\right), 1.46(\mathrm{~m}, 3 \mathrm{H}$, $\left.\mathrm{CH}_{3}\right),-0.19$ (s, 9H, $\left.\mathrm{SiMe}_{3}\right)$. 
$\underline{{ }^{13} \mathrm{C} \mathrm{NMR}}\left(101 \mathrm{MHz}, \mathrm{CDCl}_{3}\right) \delta 200.3,152.6,144.7,143.3,142.3,138.2,128.7,128.2$, $128.1,127.9,127.8,127.5,125.6,16.1,0.0$.

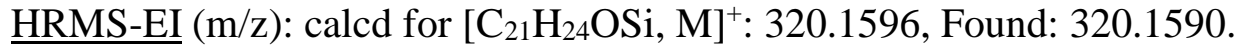

(E)-4,5-dimethyl-2-phenyl-1-(trimethylsilyl)hexa-1,4-dien-3-one (1ia)<smiles>CC(C)=C(C)C(=O)C(=CS(C)(=O)=O)c1ccccc1</smiles>

1ia

Serial number: cj-2-19, Route A was followed with corresponding dienol S4 (820 mg, $3.0 \mathrm{mmol}$ ), DMP (1.5 g, $3.6 \mathrm{mmol})$, pyridine (950 mg, $12.0 \mathrm{mmol})$ in DCM (25 mL) to afford 1ia as colorless oil (650 mg, 79\% yield for the last step). TLC $R_{f}=0.82$ $(\mathrm{PE} / \mathrm{EA}=10: 1, \mathrm{v} / \mathrm{v})$.

$\underline{{ }^{1} \mathrm{H} \mathrm{NMR}}\left(400 \mathrm{MHz}, \mathrm{CDCl}_{3}\right) \delta 7.33(\mathrm{~d}, J=5.8 \mathrm{~Hz}, 3 \mathrm{H}, \mathrm{Ar}-\mathrm{H}), 7.21-7.12(\mathrm{~m}, 2 \mathrm{H}$,

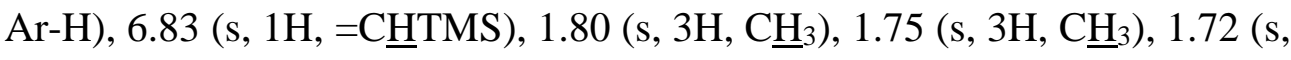
$\left.3 \mathrm{H}, \mathrm{CH}_{3}\right),-0.11$ (s, 9H, $\left.\mathrm{SiMe}_{3}\right)$.

${ }^{13} \mathrm{C} \mathrm{NMR}\left(101 \mathrm{MHz}, \mathrm{CDCl}_{3}\right) \delta 203.1,199.1,156.2,146.1,139.0,134.1,130.6,130.1$, $128.4,23.1,20.9,17.4,0.0$.

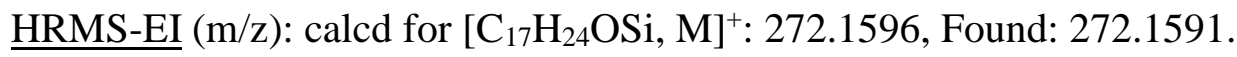

(E)-4-methyl-2-phenyl-1-(triethylsilyl)penta-1,4-dien-3-one (1ja)<smiles>C=C(C)C(=O)/C(=C/[SiH2]CCC)c1ccccc1</smiles>

$1 \mathrm{ja}$

Serial number: cj-1-173, Route A was followed with corresponding dienol S4 (1.1 g, $3.9 \mathrm{mmol})$, DMP (2.0 g, $4.7 \mathrm{mmol})$, pyridine (1.2 g, $15.6 \mathrm{mmol})$ in DCM (25 mL) to afford target product as colorless oil (915 mg, 82\% yield for the last step). TLC $R_{f}=$ $0.85(\mathrm{PE} / \mathrm{EA}=10: 1, \mathrm{v} / \mathrm{v})$. 


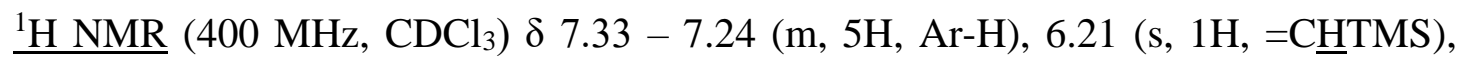
$5.92\left(\mathrm{~s}, 1 \mathrm{H},=\underline{\mathrm{C}}_{2}\right), 5.85\left(\mathrm{~s}, 1 \mathrm{H},=\mathrm{C}_{2}\right), 1.95\left(\mathrm{~s}, 3 \mathrm{H}, \underline{\mathrm{C}}_{3}\right), 0.84(\mathrm{t}, J=7.9$ $\left.\mathrm{Hz}, 9 \mathrm{H}, \mathrm{Si}\left(\mathrm{CH}_{2} \underline{\mathrm{CH}}_{3}\right)_{3}\right), 0.41\left(\mathrm{q}, J=7.9 \mathrm{~Hz}, 6 \mathrm{H}, \mathrm{Si}\left(\mathrm{C}_{2} \mathrm{CH}_{3}\right)_{3}\right)$.

${ }^{13} \mathrm{C} \mathrm{NMR}\left(101 \mathrm{MHz}, \mathrm{CDCl}_{3}\right) \delta 199.3,156.2,143.8,138.9,136.3,128.5,127.9,127.9$, $127.4,18.0,7.4,4.2$.

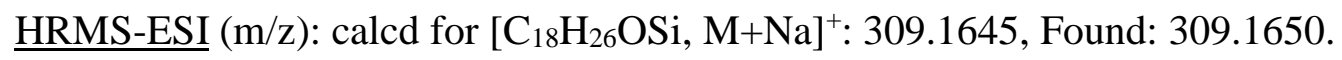

(Z)-4-methyl-2-phenyl-1-(trimethylsilyl)penta-1,4-dien-3-one (11a)<smiles>C=C(C)C(=O)/C(=C/c1ccccc1)C(N)=O</smiles>

Serial number: cj-2-5, Route $\mathrm{H}$ was followed to afford 1la as colorless oil (490 mg, 67\% yield for the last step). TLC $R_{f}=0.77(\mathrm{PE} / \mathrm{EA}=10: 1, \mathrm{v} / \mathrm{v})$.

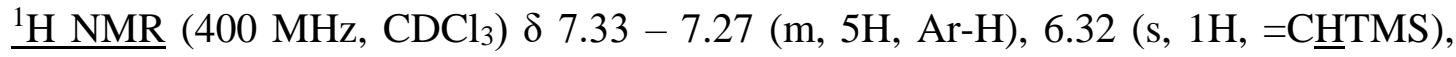
$5.94\left(\mathrm{~m}, 2 \mathrm{H},=\underline{\mathrm{C}}_{2}\right), 1.96\left(\mathrm{t}, J=1.1 \mathrm{~Hz}, 3 \mathrm{H}, \mathrm{CH}_{3}\right), 0.09$ (s, 9H, Si $\left.\underline{\mathrm{Me}}_{3}\right)$.

${ }^{13} \mathrm{C} \mathrm{NMR}\left(101 \mathrm{MHz}, \mathrm{CDCl}_{3}\right) \delta 201.8,155.5,145.0,139.7,132.3,131.5,129.2,128.8$, $126.3,17.5,0.0$.

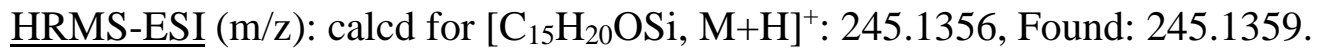




\section{Procedures of Enantioselective Silicon-Directed Nazarov Cyclization}

\subsection{A Typical Procedure}
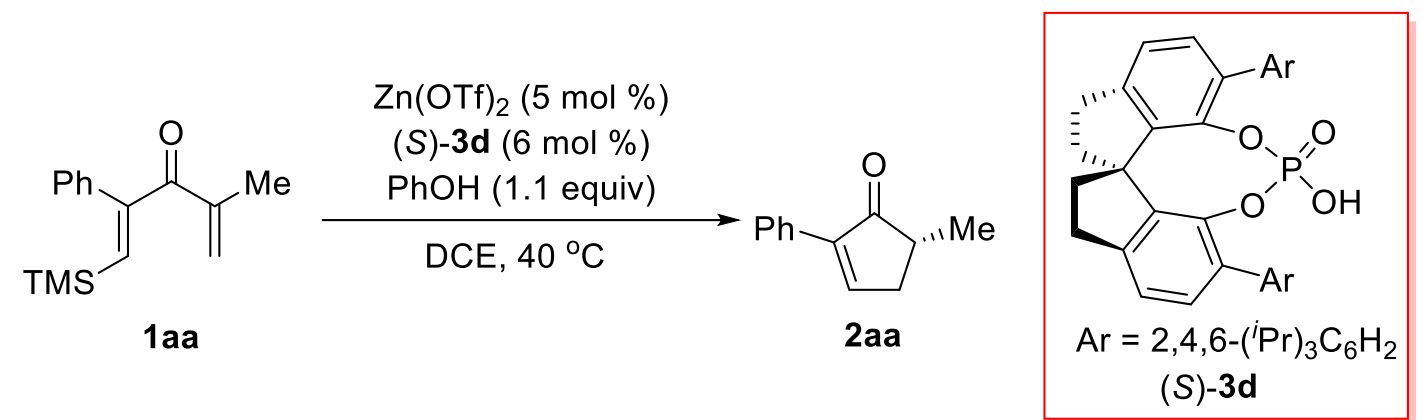

The Zn(OTf) 2 (3.6 mg, $0.01 \mathrm{mmol}, 5 \mathrm{~mol} \%)$, $(S)-3 \mathrm{~d}(8.6 \mathrm{mg}, 0.012 \mathrm{mmol}, 6$ mol \%) and $\mathrm{PhOH}(20.7 \mathrm{mg}, 0.22 \mathrm{mmol}, 1.1$ equiv) were introduced into an oven-dried Schlenk tube in an argon-filled glovebox. After $3 \mathrm{~mL}$ DCE was injected into the Schlenk tube, the mixture was stirred at $40{ }^{\circ} \mathrm{C}$. The substrate 1aa $(48.8 \mathrm{mg}$, $0.2 \mathrm{mmol}$ ) was introduced into the mixture in one portion and stirred at $40{ }^{\circ} \mathrm{C}$ for $12 \mathrm{~h}$ (the reaction progress was monitored by TLC). Then the reaction mixture was concentrated and purified by a flash chromatography on silica gel $(\mathrm{PE} / \mathrm{EA}=20: 1, \mathrm{v} / \mathrm{v})$ to give $\mathbf{2 a a}$ as a white solid.

\subsection{A Gram-Scale Experiment}

The $\mathrm{Zn}(\mathrm{OTf})_{2}(90.9 \mathrm{mg}, 0.25 \mathrm{mmol}, 5 \mathrm{~mol} \%)$ and $(S)-3 d(215.7 \mathrm{mg}, 0.3 \mathrm{mmol}$, $6 \mathrm{~mol} \%)$ were introduced into an oven-dried Schlenk flask in an argon-filled glovebox. After $75 \mathrm{~mL}$ DCE was injected into the Schlenk tube, the mixture was stirred at $40{ }^{\circ} \mathrm{C}$. 1aa $(1.220 \mathrm{~g}, 5 \mathrm{mmol})$ was introduced into the mixture in one portion and continue stirred for $16 \mathrm{~h}$ at $40{ }^{\circ} \mathrm{C}$ until the end of the reaction (monitored by TLC). The reaction mixture was concentrated and purified by a flash chromatography on silica gel $(\mathrm{PE} / \mathrm{EA}=20: 1, \mathrm{v} / \mathrm{v})$ to give $\mathbf{2 a a}$ in $92 \%$ yield $(792 \mathrm{mg}), 91 \%$ ee. $(S)-3 \mathbf{d}$ was also recovered in $95 \%$ yield (205 mg). Serial number: cj-1-158. 
Table S1. Optimization of the Reaction Conditions - Solvent effect ${ }^{a}$<smiles>C=C(C)C(=O)/C(=C\S(C)(=O)=O)c1ccccc1</smiles>

1 aa

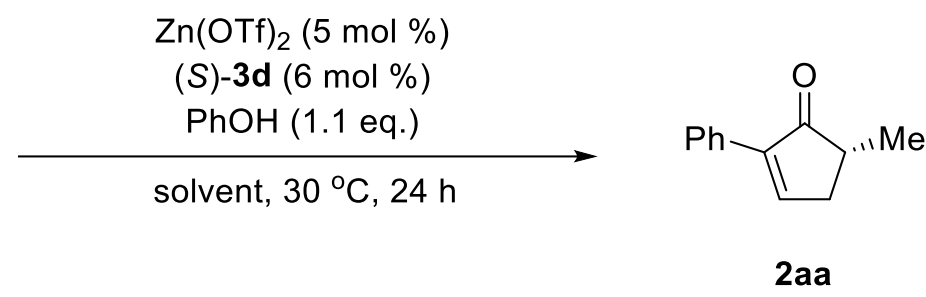

2aa

\begin{tabular}{cccc}
\hline entry & solvent & yield $(\%)^{b}$ & ee $(\%)^{c}$ \\
\hline 1 & DCE & 87 & -94 \\
2 & $\mathrm{DCM}$ & 88 & -95 \\
3 & $\mathrm{CHCl}_{3}$ & 92 & -3 \\
4 & $\mathrm{PhCl}$ & 82 & -70 \\
5 & toluene & 35 & -67 \\
6 & hexane & 39 & -82 \\
7 & $\mathrm{THF}$ & $<5$ & - \\
8 & $\mathrm{CH} \mathrm{CN}$ & $<5$ & - \\
\hline
\end{tabular}

${ }^{a}$ Reaction conditions: $\mathrm{Zn}(\mathrm{OTf})_{2} /(S)-\mathbf{3 d} / \mathbf{1 a a} / \mathrm{PhOH}=0.01: 0.012: 0.2: 0.22(\mathrm{mmol})$, in 3 $\mathrm{mL}$ of solvent at $30{ }^{\circ} \mathrm{C}, 24 \mathrm{~h} .{ }^{b}$ Isolated yield. ${ }^{c}$ Determined by chiral HPLC.

Table S2. Silicon effect on the Nazarov cyclization

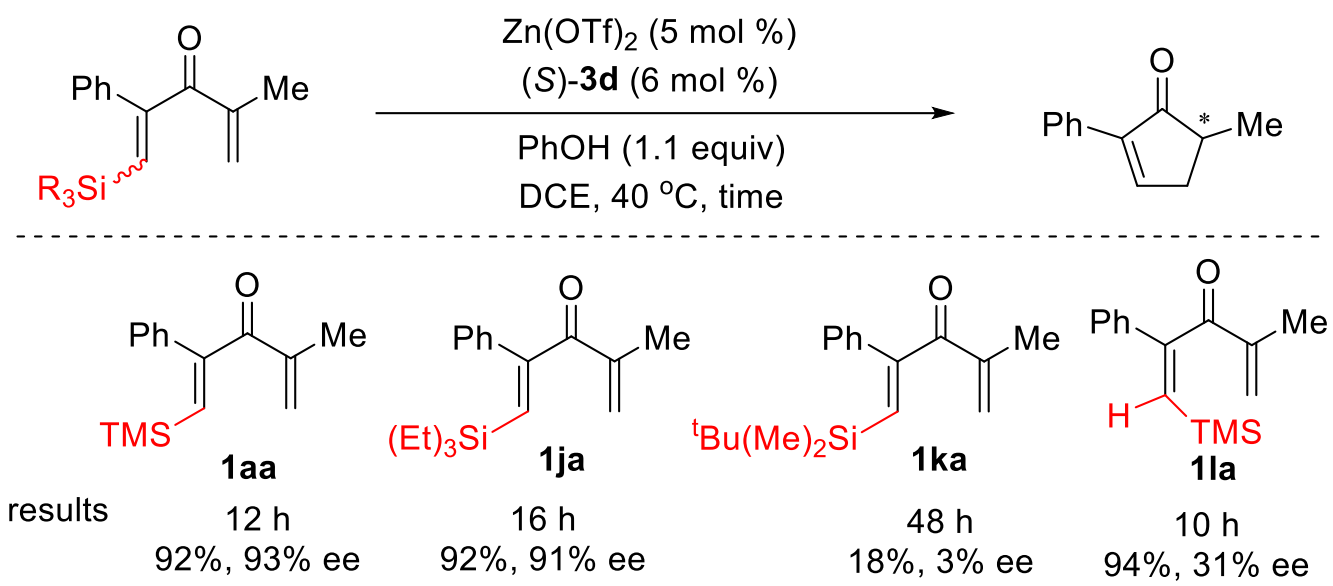

Typical Procedure: The $\mathrm{Zn}(\mathrm{OTf})_{2}(3.6 \mathrm{mg}, 0.01 \mathrm{mmol}, 5 \mathrm{~mol} \%),(S)-3 \mathbf{d}(8.6 \mathrm{mg}$, $0.012 \mathrm{mmol}, 6 \mathrm{~mol} \%)$ and $\mathrm{PhOH}(20.7 \mathrm{mg}, 0.22 \mathrm{mmol}, 1.1$ equiv) were introduced into an oven-dried Schlenk tube in an argon-filled glovebox. After $3 \mathrm{~mL}$ DCE was injected into the Schlenk tube, the mixture was stirred at $40{ }^{\circ} \mathrm{C}$. The substrate $1(0.2$ 
mmol) was introduced into the mixture in one portion and stirred at $40{ }^{\circ} \mathrm{C}$ for specific time. Then the reaction mixture was concentrated and purified by a flash chromatography on silica gel $(\mathrm{PE} / \mathrm{EA}=20: 1, \mathrm{v} / \mathrm{v})$ to give 2 . 


\section{Analytical Data of Cyclization Products}

\section{5-methyl-2-phenylcyclopent-2-en-1-one (2aa)}<smiles>CC1CC=C(c2ccccc2)C1=O</smiles>

2aa

Serial number: cj-1-103, white solid, mp: $62-64{ }^{\circ} \mathrm{C}, 92 \%$ yield $(32.0 \mathrm{mg}), 93 \%$ ee, $[\alpha]_{\mathrm{D}}^{27}=-86.8\left(c 0.47, \mathrm{CHCl}_{3}\right)$, TLC $R_{f}=0.43(\mathrm{PE} / \mathrm{EA}=10: 1, \mathrm{v} / \mathrm{v})$.

$\underline{{ }^{1} \mathrm{H} \mathrm{NMR}}\left(400 \mathrm{MHz}, \mathrm{CDCl}_{3}\right) \delta 7.81\left(\mathrm{t}, J=3.0 \mathrm{~Hz}, 1 \mathrm{H},=\mathrm{CHCH}_{2}\right), 7.78-7.64(\mathrm{~m}, 2 \mathrm{H}$, Ar-H), $7.49-7.31(\mathrm{~m}, 3 \mathrm{H}$, Ar-H), 3.00 (ddd, $J=19.5,6.7,3.1 \mathrm{~Hz}, 1 \mathrm{H}$, $\left.\mathrm{C}_{2}\right), 2.61(\mathrm{~m}, 1 \mathrm{H}, \mathrm{C} \underline{\mathrm{HCO}}), 2.34\left(\mathrm{dt}, J=19.5,2.7 \mathrm{~Hz}, 1 \mathrm{H}, \mathrm{C}_{2}\right), 1.31$ (d, $J$ $\left.=7.5 \mathrm{~Hz}, 3 \mathrm{H}, \underline{\mathrm{C}}_{3}\right)$.

${ }^{13} \mathrm{C} \mathrm{NMR}\left(101 \mathrm{MHz}, \mathrm{CDCl}_{3}\right) \delta 210.8,157.8,142.9,132.5,129.1,129.0,127.7,41.9$, 36.0, 17.3.

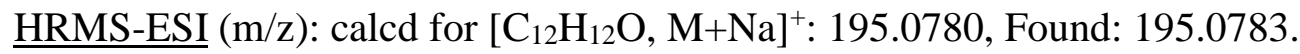

HPLC condition: Chiralpak IC-3 column, $n$-hexane/2-propanol $=98: 2$, flow rate $=1$ $\mathrm{mL} / \mathrm{min}$, wavelength $=254 \mathrm{~nm}, t_{\mathrm{R}}=12.53$ min for major isomer, $t_{\mathrm{R}}=13.86$ min for minor isomer.

The NMR spectroscopic data were in agreement with those reported in the literature. ${ }^{10}$

\section{2-(4-methoxyphenyl)-5-methylcyclopent-2-en-1-one (2ab)}<smiles>COc1ccc(C2=CCC(C)C2=O)cc1</smiles>

$2 \mathbf{a b}$

Serial number: cj-1-117, white solid, mp: $66-67{ }^{\circ} \mathrm{C} .93 \%$ yield $(37.6 \mathrm{mg}), 94 \%$ ee, $[\alpha]_{\mathrm{D}}^{25}=-77.0\left(c 0.15, \mathrm{CHCl}_{3}\right)$, TLC $R_{f}=0.33(\mathrm{PE} / \mathrm{EA}=10: 1, \mathrm{v} / \mathrm{v})$.

$\underline{{ }^{1} \mathrm{H} \mathrm{NMR}}\left(400 \mathrm{MHz}, \mathrm{CDCl}_{3}\right) \delta 7.69-7.64\left(\mathrm{~m}, 3 \mathrm{H},=\mathrm{C}_{\underline{H C H}}, \mathrm{Ar}-\mathrm{H}\right), 6.89$ (d, $J=8.8$ Hz, 2H, Ar-H), 3.80 (s, 3H, OMe), 2.93 (ddd, $J=19.4,6.7,3.1 \mathrm{~Hz}, 1 \mathrm{H}$, 
$\left.\underline{\mathrm{C}}_{2}\right), 2.54(\mathrm{~m}, 1 \mathrm{H}, \mathrm{C} \underline{\mathrm{HCO}}), 2.26\left(\mathrm{~d}, J=19.4 \mathrm{~Hz}, 1 \mathrm{H}, \underline{\mathrm{C}}_{2}\right), 1.24(\mathrm{~d}, J=7.5$ $\left.\mathrm{Hz}, 3 \mathrm{H}, \underline{\mathrm{C}}_{3}\right)$.

${ }^{13} \mathrm{C} \mathrm{NMR}\left(101 \mathrm{MHz}, \mathrm{CDCl}_{3}\right) \delta 210.4,159.7,155.3,141.5,128.3,124.5,113.8,55.3$, $41.1,35.2,16.6$.

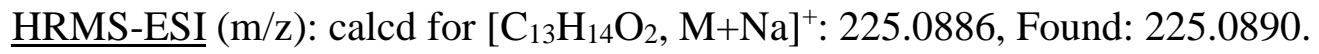

HPLC condition: Chiralpak IC-3 column, $n$-hexane/2-propanol $=95: 5$, flow rate $=1$ $\mathrm{mL} / \mathrm{min}$, wavelength $=220 \mathrm{~nm}, t_{\mathrm{R}}=16.82 \min$ for major isomer, $t_{\mathrm{R}}=22.93$ min for minor isomer.

The NMR spectroscopic data were in agreement with those reported in the literature. ${ }^{10}$

\section{5-methyl-2-(p-tolyl)cyclopent-2-en-1-one (2ac)}<smiles>Cc1ccc(C2=CCC(C)C2=O)cc1</smiles>

Serial number: cj-2-30, white solid, mp: $64-65{ }^{\circ} \mathrm{C}, 90 \%$ yield $(33.5 \mathrm{mg}), 94 \%$ ee, $[\alpha]_{\mathrm{D}}^{25}=-93.1\left(c 0.15, \mathrm{CHCl}_{3}\right)$, TLC $R_{f}=0.45(\mathrm{PE} / \mathrm{EA}=10: 1, \mathrm{v} / \mathrm{v})$.

$\left.\underline{{ }^{1} \mathrm{H} \mathrm{NMR}}\left(400 \mathrm{MHz}, \mathrm{CDCl}_{3}\right) \delta 7.73\left(\operatorname{app~t}, J=3.0 \mathrm{~Hz}, 1 \mathrm{H},=\mathrm{C}^{\mathrm{HCH}}\right)_{2}\right), 7.65-7.54(\mathrm{~m}$, 2H, Ar-H), $7.21-7.14$ (m, 2H, Ar-H), 2.95 (ddd, $J=19.4,6.7,3.1 \mathrm{~Hz}, 1 \mathrm{H}$,

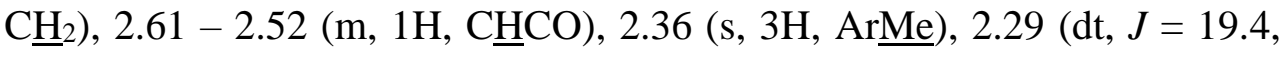
$\left.2.7 \mathrm{~Hz}, 1 \mathrm{H}, \mathrm{C}_{2}\right), 1.27$ (d, J = 7.5 Hz, 3H, $\mathrm{CH}_{3}$ ).

${ }^{13} \mathrm{C} \mathrm{NMR}\left(101 \mathrm{MHz}, \mathrm{CDCl}_{3}\right) \delta 210.3,156.3,142.0,138.2,129.1,129.0,126.9,41.2$, $35.2,21.3,16.6$.

HRMS-ESI $(\mathrm{m} / \mathrm{z})$ : calcd for $\left[\mathrm{C}_{13} \mathrm{H}_{14} \mathrm{O}, \mathrm{M}+\mathrm{Na}\right]^{+}: 209.0937$, Found: 209.0942.

$\underline{\text { HPLC }}$ condition: Chiralpak IC-3 column, $n$-hexane/2-propanol $=98: 2$, flow rate $=1$ $\mathrm{mL} / \mathrm{min}$, wavelength $=254 \mathrm{~nm}, t_{\mathrm{R}}=23.47$ min for major isomer, $t_{\mathrm{R}}=29.88$ min for minor isomer. 


\section{2-(4-fluorophenyl)-5-methylcyclopent-2-en-1-one (2ad)}<smiles>CC1CC=C(c2ccc(F)cc2)C1=O</smiles>

2ad

Serial number: cj-2-57, yellow oil, 92\% yield (35.0 mg), 90\% ee, $[\alpha]_{\mathrm{D}}{ }^{25}=-76.3(c 0.5$, $\left.\mathrm{CHCl}_{3}\right)$, TLC $R_{f}=0.32(\mathrm{PE} / \mathrm{EA}=10: 1, \mathrm{v} / \mathrm{v})$.

$\underline{{ }^{1} \mathrm{H} \text { NMR }}\left(400 \mathrm{MHz}, \mathrm{CDCl}_{3}\right) \delta 7.72\left(\mathrm{~m}, 3 \mathrm{H},=\mathrm{CHCH}_{2}, \mathrm{Ar}-\mathrm{H}\right), 7.07(\mathrm{t}, J=8.6 \mathrm{~Hz}, 2 \mathrm{H}$, Ar-H), 2.97 (ddd, $\left.J=19.6,6.8,3.1 \mathrm{~Hz}, 1 \mathrm{H}, \mathrm{C}_{2}\right), 2.57$ (td, $J=7.3,2.3 \mathrm{~Hz}$, 1H, CㅌCO), 2.30 (dt, $\left.J=19.5,2.8 \mathrm{~Hz}, 1 \mathrm{H}, \underline{\mathrm{CH}}_{2}\right), 1.27$ (d, $J=7.5 \mathrm{~Hz}, 3 \mathrm{H}$, $\left.\mathrm{CH}_{3}\right)$.

${ }^{13} \mathrm{C} \mathrm{NMR}\left(101 \mathrm{MHz}, \mathrm{CDCl}_{3}\right) \delta 210.0,162.8(\mathrm{~d}, J=248.5 \mathrm{~Hz}), 156.7,141.2,128.8(\mathrm{~d}$, $J=7.1 \mathrm{~Hz}), 127.9(\mathrm{~d}, J=3.0 \mathrm{~Hz}), 115.4(\mathrm{~d}, J=21.2 \mathrm{~Hz}), 41.1,35.2,16.5$.

HRMS-ESI (m/z): calcd for [ $\left.\mathrm{C}_{12} \mathrm{H}_{11} \mathrm{FO}, \mathrm{M}+\mathrm{Na}\right]^{+}:$213.0686, Found: 213.0690.

$\underline{\text { HPLC }}$ condition: Chiralpak IC-3 column, $n$-hexane/2-propanol $=99: 1$, flow rate $=1$ $\mathrm{mL} / \mathrm{min}$, wavelength $=254 \mathrm{~nm}, t_{\mathrm{R}}=16.52 \mathrm{~min}$ for major isomer, $t_{\mathrm{R}}=17.34$ min for minor isomer

The NMR spectroscopic data were in agreement with those reported in the literature. ${ }^{10}$

\section{2-(4-chlorophenyl)-5-methylcyclopent-2-en-1-one (2ae)}<smiles>CC1CC=C(c2ccc(Cl)cc2)C1=O</smiles>

2ae

Serial number: cj-1-156, yellow oil, $83 \%$ yield $(34.1 \mathrm{mg}), 91 \%$ ee, $[\alpha]_{\mathrm{D}}^{25}=-94.2(c$ $\left.0.2, \mathrm{CHCl}_{3}\right)$. TLC $R_{f}=0.34(\mathrm{PE} / \mathrm{EA}=10: 1, \mathrm{v} / \mathrm{v})$.

$\underline{{ }^{1} \mathrm{H} \mathrm{NMR}}\left(400 \mathrm{MHz}, \mathrm{CDCl}_{3}\right) \delta 7.78\left(\mathrm{t}, J=3.0 \mathrm{~Hz}, 1 \mathrm{H},=\mathrm{CHCH}_{2}\right), 7.72-7.61(\mathrm{~m}, 2 \mathrm{H}$, Ar-H), $7.43-7.28(\mathrm{~m}, 2 \mathrm{H}, \mathrm{Ar}-\mathrm{H}), 2.97$ (ddd, $J=19.6,6.7,3.1 \mathrm{~Hz}, 1 \mathrm{H}$, $\left.\mathrm{C}_{2}\right), 2.58(\mathrm{td}, J=7.2,2.4 \mathrm{~Hz}, 1 \mathrm{H}, \mathrm{C} \underline{\mathrm{HCO}}), 2.31(\mathrm{dt}, J=19.7,2.7 \mathrm{~Hz}, 1 \mathrm{H}$, $\left.\mathrm{C}_{2}\right), 1.27\left(\mathrm{~d}, J=7.5 \mathrm{~Hz}, 3 \mathrm{H}, \mathrm{C}_{3}\right)$.

${ }^{13} \mathrm{C} \mathrm{NMR}\left(101 \mathrm{MHz}, \mathrm{CDCl}_{3}\right) \delta 209.8,157.3,141.0,134.2,130.2,128.6,128.3,41.1$, $35.3,16.5$. 


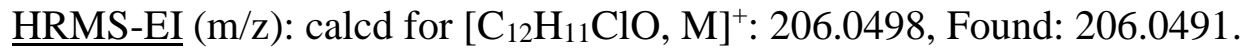

HPLC condition: Chiralpak IC-3 column, $n$-hexane/2-propanol $=98: 2$, flow rate $=1$ $\mathrm{mL} / \mathrm{min}$, wavelength $=254 \mathrm{~nm}, t_{\mathrm{R}}=9.56$ min for major isomer, $t_{\mathrm{R}}=10.26$ min for minor isomer

\section{2-(4-bromophenyl)-5-methylcyclopent-2-en-1-one (2af)}<smiles>CC1CC=C(c2ccc(Br)cc2)C1=O</smiles>

2af

Serial number: cj-2-56, yellow oil, 93\% yield $(46.5 \mathrm{mg}), 87 \%$ ee, $[\alpha]_{\mathrm{D}}{ }^{25}=-63.2(c$ $\left.0.75, \mathrm{CHCl}_{3}\right)$, TLC $R_{f}=0.31(\mathrm{PE} / \mathrm{EA}=10: 1, \mathrm{v} / \mathrm{v})$.

${ }^{1} \mathrm{H} \mathrm{NMR}\left(400 \mathrm{MHz}, \mathrm{CDCl}_{3}\right) \delta 7.78\left(\mathrm{t}, J=3.0 \mathrm{~Hz}, 1 \mathrm{H},=\mathrm{CHCH}_{2}\right), 7.65-7.57(\mathrm{~m}, 2 \mathrm{H}$, Ar-H), $7.55-7.41(\mathrm{~m}, 2 \mathrm{H}$, Ar-H), 2.96 (ddd, $J=19.6,6.7,3.1 \mathrm{~Hz}, 1 \mathrm{H}$, $\left.\mathrm{C}_{2}\right), 2.57(\mathrm{td}, J=7.2,2.5 \mathrm{~Hz}, 1 \mathrm{H}, \mathrm{C} \underline{\mathrm{HCO}}), 2.30(\mathrm{dt}, J=19.6,2.7 \mathrm{~Hz}, 1 \mathrm{H}$, $\left.\mathrm{C}_{2}\right), 1.27\left(\mathrm{~d}, J=7.7 \mathrm{~Hz}, 3 \mathrm{H}, \mathrm{C}_{3}\right)$.

${ }^{13} \mathrm{C} \mathrm{NMR}\left(101 \mathrm{MHz}, \mathrm{CDCl}_{3}\right) \delta 209.7,157.3,141.1,131.6,130.7,128.6,122.5,41.1$, $35.3,16.5$.

HRMS-EI (m/z): calcd for [ $\left.\mathrm{C}_{12} \mathrm{H}_{11} \mathrm{BrO}, \mathrm{M}\right]^{+}:$249.9993, Found: 249.9985.

$\underline{\text { HPLC }}$ condition: Chiralpak IC-3 column, $n$-hexane/2-propanol $=99: 1$, flow rate $=1$ $\mathrm{mL} / \mathrm{min}$, wavelength $=254 \mathrm{~nm}, t_{\mathrm{R}}=18.56 \mathrm{~min}$ for major isomer, $t_{\mathrm{R}}=21.85$ min for minor isomer

\section{5-methyl-2-(4-(trifluoromethyl)phenyl)cyclopent-2-en-1-one (2ag)}<smiles>CC1CC=C(c2ccc(C(F)(F)F)cc2)C1=O</smiles>

2ag

Serial number: cj-3-45, white solid, mp: $42-44{ }^{\circ} \mathrm{C}, 92 \%$ yield $(44.2 \mathrm{mg}), 91 \%$ ee, $[\alpha]_{\mathrm{D}}^{25}=-57.6\left(c 0.5, \mathrm{CHCl}_{3}\right)$, TLC $R_{f}=0.35(\mathrm{PE} / \mathrm{EA}=10: 1, \mathrm{v} / \mathrm{v})$. 
${ }^{1} \mathrm{H}$ NMR $\left(400 \mathrm{MHz}, \mathrm{CDCl}_{3}\right) \delta 7.91-7.81\left(\mathrm{~m}, 3 \mathrm{H},=\mathrm{CHCH}_{2}, \mathrm{Ar}-\mathrm{H}\right), 7.64(\mathrm{~d}, J=8.0$

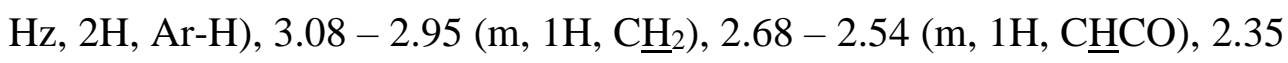
(d, $\left.J=19.7 \mathrm{~Hz}, 1 \mathrm{H}, \underline{\mathrm{CH}}_{2}\right), 1.29$ (d, $\left.J=7.5 \mathrm{~Hz}, 3 \mathrm{H}, \mathrm{C}_{3}\right)$.

${ }^{13} \mathrm{C} \mathrm{NMR}\left(101 \mathrm{MHz}, \mathrm{CDCl}_{3}\right) \delta 209.5,158.8,141.1,135.2,130.2(\mathrm{q}, J=32.3 \mathrm{~Hz})$, 127.3, $125.3(\mathrm{q}, J=3.0 \mathrm{~Hz}), 124.1(\mathrm{q}, \mathrm{J}=272.7 \mathrm{~Hz}), 41.2,35.4$, 16.4 .

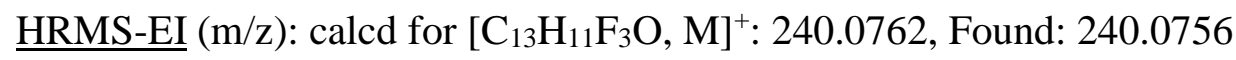

$\underline{\text { HPLC }}$ condition: Chiralpak IC-3 column, $n$-hexane/2-propanol $=98: 2$, flow rate $=1$ $\mathrm{mL} / \mathrm{min}$, wavelength $=254 \mathrm{~nm}, t_{\mathrm{R}}=8.50 \min$ for minor isomer, $t_{\mathrm{R}}=8.74$ min for major isomer.

\section{5-methyl-2-(m-tolyl)cyclopent-2-en-1-one (2ah)}

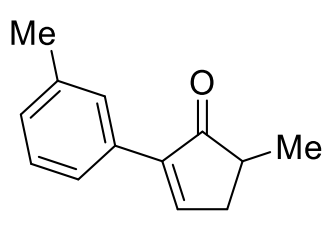

2ah

Serial number: cj-2-31, white solid, mp: $55-56{ }^{\circ} \mathrm{C}, 93 \%$ yield $(34.6 \mathrm{mg}), 95 \%$ ee, $[\alpha]_{\mathrm{D}}^{25}=-83.2\left(c 0.1, \mathrm{CHCl}_{3}\right)$, TLC $R_{f}=0.44(\mathrm{PE} / \mathrm{EA}=10: 1, \mathrm{v} / \mathrm{v})$.

${ }^{1} \mathrm{H}$ NMR $\left(400 \mathrm{MHz}, \mathrm{CDCl}_{3}\right) \delta 7.77\left(\mathrm{t}, J=3.1 \mathrm{~Hz}, 1 \mathrm{H},=\mathrm{CHCH}_{2}\right), 7.66-7.40(\mathrm{~m}, 2 \mathrm{H}$, Ar-H), $7.32-7.28$ (m, 1H, Ar-H), 7.17 (d, J=7.6 Hz, 1H, Ar-H), 2.98 (ddd, $\left.J=19.5,6.7,3.0 \mathrm{~Hz}, 1 \mathrm{H}, \mathrm{C}_{2}\right), 2.66-2.53(\mathrm{~m}, 1 \mathrm{H}, \mathrm{C} \underline{\mathrm{HCO}}), 2.40(\mathrm{~s}, 3 \mathrm{H}$,

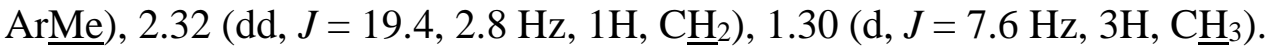

${ }^{13} \mathrm{C} \mathrm{NMR}\left(101 \mathrm{MHz}, \mathrm{CDCl}_{3}\right) \delta 210.1,157.0,142.4,138.0,131.8,129.1,128.3,127.7$, 124.1, 41.2, 35.2, 21.5, 16.6 .

$\underline{\text { HRMS-EI }}(\mathrm{m} / \mathrm{z})$ : calcd for $\left[\mathrm{C}_{13} \mathrm{H}_{14} \mathrm{O}, \mathrm{M}\right]^{+}:$: 186.1045, Found: 186.1038.

$\underline{\text { HPLC }}$ condition: Chiralpak IC-3 column, $n$-hexane/2-propanol $=98: 2$, flow rate $=1$ $\mathrm{mL} / \mathrm{min}$, wavelength $=254 \mathrm{~nm}, t_{\mathrm{R}}=12.05 \min$ for major isomer, $t_{\mathrm{R}}=13.95$ min for minor isomer 


\section{5-methyl-2-(o-tolyl)cyclopent-2-en-1-one (2ai)}<smiles>Cc1ccccc1C1=CCC(C)C1=O</smiles>

2ai

Serial number: cj-3-44, colorless oil, $91 \%$ yield $(33.9 \mathrm{mg}), 90 \%$ ee, $[\alpha]_{\mathrm{D}}{ }^{25}=-72.3(c$ $\left.0.1, \mathrm{CHCl}_{3}\right)$, TLC $R_{f}=0.42(\mathrm{PE} / \mathrm{EA}=10: 1, \mathrm{v} / \mathrm{v})$.

매 NMR $\left(400 \mathrm{MHz}, \mathrm{CDCl}_{3}\right) \delta 7.53\left(\mathrm{t}, J=2.9 \mathrm{~Hz}, 1 \mathrm{H},=\mathrm{CHCH}_{2}\right), 7.25-7.09(\mathrm{~m}, 4 \mathrm{H}$, Ar-H), $3.10-2.94\left(\mathrm{~m}, 1 \mathrm{H}, \underline{\mathrm{C}}_{2}\right), 2.57$ (m, 1H, C

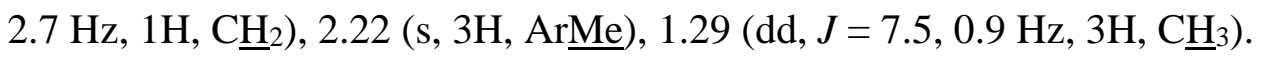
${ }^{13} \mathrm{C} \mathrm{NMR}\left(101 \mathrm{MHz}, \mathrm{CDCl}_{3}\right) \delta 209.9,159.2,145.1,136.3,132.1,130.2,129.3,128.2$, $125.6,40.2,35.9,20.2,16.7$.

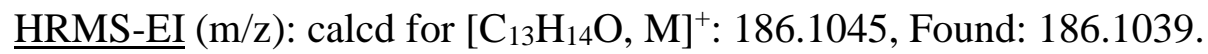

$\underline{\text { HPLC }}$ condition: Chiralpak IC-3 column, $n$-hexane/2-propanol $=98: 2$, flow rate $=1$ $\mathrm{mL} / \mathrm{min}$, wavelength $=254 \mathrm{~nm}, t_{\mathrm{R}}=27.09 \min$ for minor isomer, $t_{\mathrm{R}}=29.77$ min for major isomer.

\section{2-(benzo[d][1,3]dioxol-5-yl)-5-methylcyclopent-2-en-1-one (2aj)}

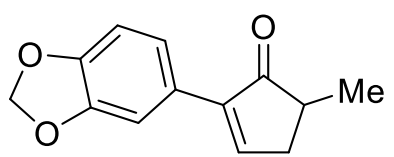

2aj

Serial number: cj-1-198, white solid, mp: $62-64{ }^{\circ} \mathrm{C}, 93 \%$ yield $(40.1 \mathrm{mg}), 96 \%$ ee, $[\alpha]_{\mathrm{D}}^{25}=-73.2\left(c 0.2, \mathrm{CHCl}_{3}\right)$, TLC $R_{f}=0.35(\mathrm{PE} / \mathrm{EA}=10: 1, \mathrm{v} / \mathrm{v})$.

${ }^{1} \mathrm{H}$ NMR $\left(400 \mathrm{MHz}, \mathrm{CDCl}_{3}\right) \delta 7.66\left(\mathrm{t}, J=3.0 \mathrm{~Hz}, 1 \mathrm{H},=\mathrm{CHCH}_{2}\right), 7.28(\mathrm{~m}, 1 \mathrm{H}, \mathrm{Ar}-\mathrm{H})$, $7.23(\mathrm{~d}, J=1.7 \mathrm{~Hz}, 1 \mathrm{H}, \mathrm{Ar}-\mathrm{H}), 6.83(\mathrm{~d}, J=8.1 \mathrm{~Hz}, 1 \mathrm{H}, \mathrm{Ar}-\mathrm{H}), 5.97$ (s, 2H, $\left.\mathrm{OC} \underline{\mathrm{H}}_{2} \mathrm{O}\right), 2.94\left(\mathrm{ddd}, J=19.5,6.7,3.1 \mathrm{~Hz}, 1 \mathrm{H}, \mathrm{C}_{2}\right), 2.56(\mathrm{td}, J=7.1,2.4$ $\mathrm{Hz}, 1 \mathrm{H}, \mathrm{C} \underline{\mathrm{HCO}}), 2.28$ (dt, $\left.J=19.5,2.7 \mathrm{~Hz}, 1 \mathrm{H}, \underline{\mathrm{CH}}_{2}\right), 1.26(\mathrm{~d}, J=7.4 \mathrm{~Hz}$, $\left.3 \mathrm{H}, \mathrm{C}_{3}\right)$.

${ }^{13} \mathrm{C} \mathrm{NMR}\left(101 \mathrm{MHz}, \mathrm{CDCl}_{3}\right) \delta 210.1,155.8,147.6,147.6,141.5,125.8,120.9,108.3$, 107.4, 101.0, 41.2, 35.1, 16.6. 


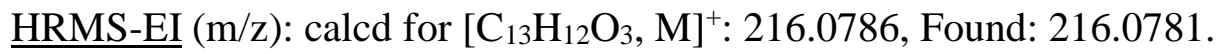

HPLC condition: Chiralpak IC-3 column, $n$-hexane/2-propanol $=99: 1$, flow rate $=1$ $\mathrm{mL} / \mathrm{min}$, wavelength $=254 \mathrm{~nm}, t_{\mathrm{R}}=46.04$ min for major isomer, $t_{\mathrm{R}}=48.36$ min for minor isomer.

\section{5-methyl-2-(naphthalen-2-yl)cyclopent-2-en-1-one (2ak)}<smiles>CC1CC=C(c2ccc3ccccc3c2)C1=O</smiles>

2ak

Serial number: cj-1-192, white solid, mp: $57-58{ }^{\circ} \mathrm{C}, 95 \%$ yield $(42.0 \mathrm{mg}), 93 \%$ ee, $[\alpha]_{\mathrm{D}}^{25}=-108.4\left(c 0.5, \mathrm{CHCl}_{3}\right)$, TLC $R_{f}=0.35(\mathrm{PE} / \mathrm{EA}=10: 1, \mathrm{v} / \mathrm{v})$.

${ }^{1} \mathrm{H}$ NMR $\left(400 \mathrm{MHz}, \mathrm{CDCl}_{3}\right) \delta 8.49-8.36(\mathrm{~m}, 1 \mathrm{H}, \mathrm{Ar}-\mathrm{H}), 7.93(\mathrm{~m}, 2 \mathrm{H}, \mathrm{Ar}-\mathrm{H}$, $\left.=\mathrm{CHCH}_{2}\right), 7.86(\mathrm{~d}, J=8.8 \mathrm{~Hz}, 2 \mathrm{H}, \mathrm{Ar}-\mathrm{H}), 7.76(\mathrm{~d}, J=1.7 \mathrm{~Hz}, 1 \mathrm{H}, \mathrm{Ar}-\mathrm{H})$, $7.54-7.46$ (m, 2H, Ar-H), 3.04 (ddd, $\left.J=19.5,6.7,3.1 \mathrm{~Hz}, 1 \mathrm{H}, \underline{\mathrm{C}}_{2}\right), 2.66$ (m, 1H, $\underline{\mathrm{HCO}}), 2.38\left(\mathrm{~d}, J=19.5 \mathrm{~Hz}, 1 \mathrm{H}, \underline{\mathrm{C}}_{2}\right), 1.34(\mathrm{~d}, J=7.4 \mathrm{~Hz}, 3 \mathrm{H}$, $\left.\mathrm{C}_{3}\right)$.

${ }^{13} \mathrm{C}$ NMR $\left(101 \mathrm{MHz}, \mathrm{CDCl}_{3}\right) \delta 210.3,157.4,141.8,133.3,133.1,129.1,128.6,128.0$, $127.6,126.4,126.3,126.2,124.7,41.3,35.3,16.6$.

$\underline{\text { HPLC }}$ condition: Chiralpak IC-3 column, $n$-hexane/2-propanol $=98: 2$, flow rate $=1$ $\mathrm{mL} / \mathrm{min}$, wavelength $=254 \mathrm{~nm}, t_{\mathrm{R}}=18.77 \mathrm{~min}$ for major isomer, $t_{\mathrm{R}}=28.54$ min for minor isomer.

The NMR spectroscopic data were in agreement with those reported in the literature. ${ }^{10}$

\section{5-methyl-2-(thiophen-2-yl)cyclopent-2-en-1-one (2al)}<smiles>CC1CC=C(c2cccs2)C1=O</smiles>

2al

Serial number: cj-1-157, yellow oil, $75 \%$ yield $(26.7 \mathrm{mg}), 91 \%$ ee, $[\alpha]_{\mathrm{D}}^{25}=-67.2(c 1$, $\left.\mathrm{CHCl}_{3}\right), \mathrm{TLC} R_{f}=0.37(\mathrm{PE} / \mathrm{EA}=10: 1, \mathrm{v} / \mathrm{v})$. 
$\underline{{ }^{1} \mathrm{H} \mathrm{NMR}}\left(400 \mathrm{MHz}, \mathrm{CDCl}_{3}\right) \delta 7.67\left(\mathrm{t}, J=3.1 \mathrm{~Hz}, 1 \mathrm{H},=\mathrm{CHCH}_{2}\right), 7.63(\mathrm{~d}, J=3.6 \mathrm{~Hz}$, 1H, Ar-H), 7.28 (m, 1H, Ar-H), 7.04 (m, 1H, Ar-H), 2.97 (ddd, $J=19.8,6.7$, $\left.3.3 \mathrm{~Hz}, 1 \mathrm{H}, \underline{\mathrm{C}}_{2}\right), 2.55$ (d, $\left.J=2.4 \mathrm{~Hz}, 1 \mathrm{H}, \mathrm{C} \underline{\mathrm{HCO}}\right), 2.30$ (d, $J=19.8 \mathrm{~Hz}, 1 \mathrm{H}$, $\left.\mathrm{C}_{2}\right), 1.25\left(\mathrm{~d}, J=7.5 \mathrm{~Hz}, 3 \mathrm{H}, \underline{\mathrm{CH}}_{3}\right)$.

${ }^{13} \mathrm{C} \mathrm{NMR}\left(101 \mathrm{MHz}, \mathrm{CDCl}_{3}\right) \delta 208.7,153.5,136.6,133.3,127.2,125.8,125.6,40.7$, $35.5,16.4$.

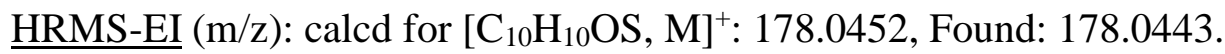

$\underline{\text { HPLC }}$ condition: Chiralpak IC-3 column, $n$-hexane/2-propanol $=98: 2$, flow rate $=1$ $\mathrm{mL} / \mathrm{min}$, wavelength $=254 \mathrm{~nm}, t_{\mathrm{R}}=9.56 \mathrm{~min}$ for major isomer, $t_{\mathrm{R}}=10.26$ min for minor isomer.

\section{5-ethyl-2-phenylcyclopent-2-en-1-one (2ba) ${ }^{10}$}

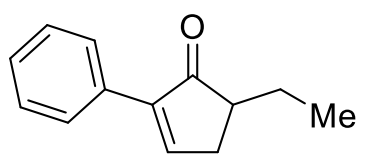

$2 \mathrm{ba}$

Serial number: cj-2-51, white solid, mp: $39-40{ }^{\circ} \mathrm{C}, 92 \%$ yield $(34.0 \mathrm{mg}), 95 \%$ ee, $[\alpha]_{\mathrm{D}}^{25}=-98.8\left(c 1, \mathrm{CHCl}_{3}\right)$, TLC $R_{f}=0.44(\mathrm{PE} / \mathrm{EA}=10: 1, \mathrm{v} / \mathrm{v})$.

${ }^{1} \mathrm{H}$ NMR $\left(400 \mathrm{MHz}, \mathrm{CDCl}_{3}\right) \delta 7.79\left(\mathrm{t}, J=3.0 \mathrm{~Hz}, 1 \mathrm{H},=\mathrm{CHCH}_{2}\right), 7.76-7.59(\mathrm{~m}, 2 \mathrm{H}$, Ar-H), $7.44-7.28(\mathrm{~m}, 3 \mathrm{H}$, Ar-H), 2.88 (ddd, $J=19.5,6.6,3.0 \mathrm{~Hz}, 1 \mathrm{H}$, $\left.\mathrm{C}_{2}\right), 2.54-2.45(\mathrm{~m}, 1 \mathrm{H}, \mathrm{C} \underline{\mathrm{HCO}}), 2.39\left(\mathrm{dt}, J=19.5,2.7 \mathrm{~Hz}, 1 \mathrm{H}, \underline{\mathrm{CH}}_{2}\right)$, 1.93 (ddd, $J=13.8,7.4,4.6 \mathrm{~Hz}, 1 \mathrm{H}, \mathrm{CH}_{2} \mathrm{CH}_{3}$ ), 1.52 (ddd, $J=13.7,8.8,6.9$ $\left.\mathrm{Hz}, 1 \mathrm{H}, \mathrm{C}_{2} \mathrm{CH}_{3}\right), 1.00\left(\mathrm{t}, J=7.4 \mathrm{~Hz}, 3 \mathrm{H}, \mathrm{CH}_{2} \underline{\mathrm{C}}_{3}\right)$.

${ }^{13} \mathrm{C} \mathrm{NMR}\left(101 \mathrm{MHz}, \mathrm{CDCl}_{3}\right) \delta 209.5,157.5,142.9,131.8,128.4,128.3,127.0,47.9$, $32.7,24.6,11.4$.

$\underline{\text { HPLC }}$ condition: Chiralpak IC-3 column, $n$-hexane/2-propanol $=99: 1$, flow rate $=1$ $\mathrm{mL} / \mathrm{min}$, wavelength $=254 \mathrm{~nm}, t_{\mathrm{R}}=20.94$ min for major isomer, $t_{\mathrm{R}}=22.28$ min for minor isomer. 


\section{2-phenyl-5-propylcyclopent-2-en-1-one (2bb)}<smiles>CCCC1CC=C(c2ccccc2)C1=O</smiles>

2bb

Serial number: cj-2-52, colorless oil, 94\% yield $(37.6 \mathrm{mg}), 83 \%$ ee, $[\alpha]_{\mathrm{D}}{ }^{25}=-81.2(c 1$, $\left.\mathrm{CHCl}_{3}\right)$, TLC $R_{f}=0.45(\mathrm{PE} / \mathrm{EA}=10: 1, \mathrm{v} / \mathrm{v})$.

$\underline{{ }^{1} \mathrm{H} \mathrm{NMR}}\left(400 \mathrm{MHz}, \mathrm{CDCl}_{3}\right) \delta 7.81\left(\mathrm{t}, J=3.1 \mathrm{~Hz}, 1 \mathrm{H},=\mathrm{CHCH}_{2}\right), 7.73(\mathrm{~d}, J=7.5 \mathrm{~Hz}$, 2H, Ar-H), 7.38 (m, 3H, Ar-H), 2.91 (ddd, $\left.J=19.5,6.7,3.0 \mathrm{~Hz}, 1 \mathrm{H}, \underline{\mathrm{C}}_{2}\right)$, $2.56(\mathrm{~d}, J=7.0 \mathrm{~Hz}, 1 \mathrm{H}, \mathrm{C} \underline{\mathrm{HCO}}), 2.41\left(\mathrm{dd}, J=19.5,2.8 \mathrm{~Hz}, 1 \mathrm{H}, \underline{\mathrm{C}}_{2}\right), 1.95$ - $1.84\left(\mathrm{~m}, 1 \mathrm{H}, \underline{\mathrm{CH}}_{2} \mathrm{CH}_{2} \mathrm{CH}_{3}\right), 1.46\left(\mathrm{~m}, 3 \mathrm{H}, \underline{\mathrm{CH}}_{2} \underline{\mathrm{CH}}_{2} \mathrm{CH}_{3}\right), 0.99$ (t, $J=6.8$ $\left.\mathrm{Hz}, 3 \mathrm{H}, \mathrm{CH}_{2} \mathrm{CH}_{2} \mathrm{C}_{3}\right)$.

${ }^{13} \mathrm{C} \mathrm{NMR}\left(101 \mathrm{MHz}, \mathrm{CDCl}_{3}\right) \delta 209.6,157.4,142.7,131.9,128.4,128.3,127.0,46.5$, $33.8,33.2,20.5,14.1$.

HRMS-ESI $(\mathrm{m} / \mathrm{z})$ : calcd for $\left[\mathrm{C}_{14} \mathrm{H}_{16} \mathrm{O}, \mathrm{M}+\mathrm{Na}\right]^{+}:$223.1093, Found: 223.1098 .

$\underline{\text { HPLC }}$ condition: Chiralpak IC-3 column, $n$-hexane/2-propanol $=99.3: 0.7$, flow rate $=$ $1 \mathrm{~mL} / \mathrm{min}$, wavelength $=254 \mathrm{~nm}, t_{\mathrm{R}}=30.68 \mathrm{~min}$ for major isomer, $t_{\mathrm{R}}=$ $33.20 \mathrm{~min}$ for minor isomer.

\section{5-hexyl-2-phenylcyclopent-2-en-1-one (2bc)}

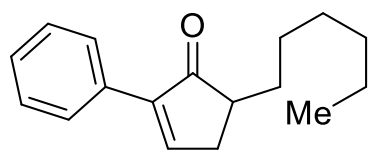

2bc

Serial number: cj-1-132, colorless oil, $89 \%$ yield $(43.0 \mathrm{mg}), 69 \%$ ee, $[\alpha]_{\mathrm{D}}{ }^{25}=-85.8(c$ $\left.1, \mathrm{CHCl}_{3}\right)$, TLC $R_{f}=0.56(\mathrm{PE} / \mathrm{EA}=10: 1, \mathrm{v} / \mathrm{v})$.

$\underline{{ }^{1} \mathrm{H} \mathrm{NMR}}\left(400 \mathrm{MHz}, \mathrm{CDCl}_{3}\right) \delta 7.78\left(\mathrm{t}, J=3.3 \mathrm{~Hz}, 1 \mathrm{H},=\mathrm{CHCH}_{2}\right), 7.70(\mathrm{~d}, J=7.5 \mathrm{~Hz}$, 2H, Ar-H), 7.36 (m, 3H, Ar-H), 2.88 (ddd, $J=19.7,6.7,3.1 \mathrm{~Hz}, 1 \mathrm{H}, \underline{\mathrm{C}}_{2}$ ), $2.52(\mathrm{t}, J=7.4 \mathrm{~Hz}, 1 \mathrm{H}, \mathrm{C} \underline{\mathrm{HCO}}), 2.38\left(\mathrm{~d}, J=19.5 \mathrm{~Hz}, 1 \mathrm{H}, \underline{\mathrm{C}}_{2}\right), 1.89$ (t, $J=$ $\left.6.6 \mathrm{~Hz}, 1 \mathrm{H}, \mathrm{C}_{2}\left(\mathrm{CH}_{2}\right)_{4} \mathrm{CH}_{3}\right), 1.44-1.24\left(\mathrm{~m}, 9 \mathrm{H}, \underline{\mathrm{C}}_{2}\left(\mathrm{C}_{2}\right)_{4} \mathrm{CH}_{3}\right), 0.89$ (t, J $\left.=6.4 \mathrm{~Hz}, 3 \mathrm{H},\left(\mathrm{CH}_{2}\right)_{5} \mathrm{C}_{3}\right)$. 
${ }^{13} \mathrm{C} \mathrm{NMR}\left(101 \mathrm{MHz}, \mathrm{CDCl}_{3}\right) \delta 209.8,157.6,142.7,131.8,128.4,128.3,127.0,46.7$,

$33.3,31.7,31.7,29.3,27.2,22.6,14.1$.

HRMS-ESI (m/z): calcd for $\left[\mathrm{C}_{17} \mathrm{H}_{22} \mathrm{O}, \mathrm{M}+\mathrm{Na}\right]^{+}: 265.1563$, Found: 265.1568.

HPLC condition: Chiralpak ID-3 column, $n$-hexane/2-propanol $=99: 1$, flow rate $=1$ $\mathrm{mL} / \mathrm{min}$, wavelength $=254 \mathrm{~nm}, t_{\mathrm{R}}=7.80 \mathrm{~min}$ for minor isomer, $t_{\mathrm{R}}=8.06$ min for major isomer.

\section{5-isopropyl-2-phenylcyclopent-2-en-1-one (2bd)}<smiles>CC(C)C1CC=C(c2ccccc2)C1=O</smiles>

2bd

Serial number: cj-2-198, colorless oil, $82 \%$ yield $(32.7 \mathrm{mg}), 97 \%$ ee, $[\alpha]_{\mathrm{D}}{ }^{25}=-148.8$ $\left(c 1, \mathrm{CHCl}_{3}\right)$, TLC $R_{f}=0.41(\mathrm{PE} / \mathrm{EA}=10: 1, \mathrm{v} / \mathrm{v})$.

$\underline{{ }^{1} \mathrm{H} \mathrm{NMR}}\left(400 \mathrm{MHz}, \mathrm{CDCl}_{3}\right) \delta 7.81\left(\mathrm{t}, J=3.1 \mathrm{~Hz}, 1 \mathrm{H},=\mathrm{C}_{\mathbf{H C H}}\right), 7.70(\mathrm{~d}, J=7.5 \mathrm{~Hz}$, 2H, Ar-H), 7.36 (m, 3H, Ar-H), 2.70 (ddd, $\left.J=19.5,6.6,3.1 \mathrm{~Hz}, 1 \mathrm{H}, \underline{\mathrm{C}}_{2}\right)$, $2.60-2.42\left(\mathrm{~m}, 2 \mathrm{H}, \mathrm{C} \underline{\mathrm{HCO}}, \underline{\mathrm{C}}_{2}\right), 2.36\left(\mathrm{~m}, 1 \mathrm{H}, \underline{\mathrm{CMMe}}_{2}\right), 1.03$ (d, $J=6.9$ $\left.\mathrm{Hz}, 3 \mathrm{H}, \mathrm{CHMe}_{2}\right), 0.81$ (d, J=6.8 Hz, 3H, $\left.\mathrm{CHMe}_{2}\right)$.

${ }^{13} \mathrm{C} \mathrm{NMR}\left(101 \mathrm{MHz}, \mathrm{CDCl}_{3}\right) \delta 209.4,158.1,143.6,131.8,128.4,128.3,127.0,52.2$, 29.1, 28.7, 20.8, 17.0.

$\underline{\text { HPLC }}$ condition: Chiralpak IC-3 column, $n$-hexane/2-propanol $=99: 1$, flow rate $=1$ $\mathrm{mL} / \mathrm{min}$, wavelength $=254 \mathrm{~nm}, t_{\mathrm{R}}=11.16 \mathrm{~min}$ for minor isomer, $t_{\mathrm{R}}=11.95$ min for major isomer.

The NMR spectroscopic data were in agreement with those reported in the literature. ${ }^{10}$

\section{5-cyclopropyl-2-phenylcyclopent-2-en-1-one (2be)}

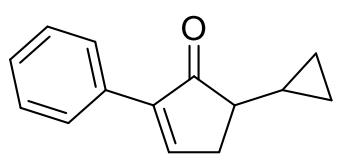

2be

Serial number: cj-3-54, white solid, mp: $50-51{ }^{\circ} \mathrm{C}, 86 \%$ yield $(34.0 \mathrm{mg}), 90 \%$ ee, $[\alpha]_{\mathrm{D}}^{28}=-39.3\left(c 0.55, \mathrm{CHCl}_{3}\right)$, TLC $R_{f}=0.40(\mathrm{PE} / \mathrm{EA}=10: 1, \mathrm{v} / \mathrm{v})$. 
$\underline{{ }^{1} \mathrm{H} \mathrm{NMR}}\left(400 \mathrm{MHz}, \mathrm{CDCl}_{3}\right) \delta 7.77\left(\mathrm{t}, J=3.0 \mathrm{~Hz}, 1 \mathrm{H},=\mathrm{CHCH}_{2}\right), 7.72(\mathrm{~d}, J=7.5 \mathrm{~Hz}$, 2H, Ar-H), 7.36 (m, 3H, Ar-H), 2.84 (ddd, $J=19.6,6.8,3.0 \mathrm{~Hz}, 1 \mathrm{H}, \mathrm{C}_{2}$ ), $2.38(\mathrm{dt}, J=19.6,2.7 \mathrm{~Hz}, 1 \mathrm{H}, \mathrm{C} \underline{\mathrm{HCO}}), 2.21\left(\mathrm{td}, J=7.4,2.4 \mathrm{~Hz}, 1 \mathrm{H}, \underline{\mathrm{C}}_{2}\right)$, $0.98\left(\mathrm{dq}, J=10.8,4.0,3.1 \mathrm{~Hz}, 1 \mathrm{H}, \mathrm{C} \underline{\mathrm{H}}\left(\mathrm{CH}_{2}\right)_{2}\right), 0.62$ (tt, $J=9.1,4.7 \mathrm{~Hz}, 1 \mathrm{H}$, $\left.\left.\left.\mathrm{CH}\left(\mathrm{C}_{2}\right)_{2}\right)\right), 0.48\left(\mathrm{ddq}, J=17.7,8.8,4.7,4.0 \mathrm{~Hz}, 2 \mathrm{H}, \mathrm{CH}\left(\mathrm{C}_{2}\right)_{2}\right)\right), 0.25(\mathrm{dq}$, $\left.\left.J=9.7,5.1,4.6 \mathrm{~Hz}, 1 \mathrm{H}, \mathrm{CH}\left(\mathrm{C}_{2}\right)_{2}\right)\right)$.

${ }^{13} \mathrm{C} \mathrm{NMR}\left(101 \mathrm{MHz}, \mathrm{CDCl}_{3}\right) \delta 207.2,155.7,141.2,130.4,127.0,126.9,125.6,47.8$, $30.9,11.5,1.6,0.0$.

HRMS-ESI $(\mathrm{m} / \mathrm{z})$ : calcd for $\left[\mathrm{C}_{14} \mathrm{H}_{14} \mathrm{O}, \mathrm{M}+\mathrm{Na}\right]^{+}:$221.0937, Found: 221.0942.

$\underline{\text { HPLC }}$ condition: Chiralpak IC-3 column, $n$-hexane/2-propanol $=98: 2$, flow rate $=1$ $\mathrm{mL} / \mathrm{min}$, wavelength $=254 \mathrm{~nm}, t_{\mathrm{R}}=24.66 \mathrm{~min}$ for major isomer, $t_{\mathrm{R}}=28.99$ min for minor isomer.

\section{5-(but-3-en-1-yl)-2-phenylcyclopent-2-en-1-one (2bf)}

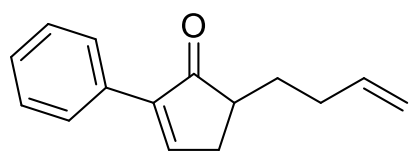

$2 b f$

Serial number: cj-3-46, colorless oil, 73\% yield $(30.9 \mathrm{mg}), 89 \%$ ee, $[\alpha]_{\mathrm{D}}^{25}=-97.4(c$ $\left.0.38, \mathrm{CHCl}_{3}\right)$, TLC $R_{f}=0.43(\mathrm{PE} / \mathrm{EA}=10: 1, \mathrm{v} / \mathrm{v})$.

${ }^{1} \mathrm{H}$ NMR $\left(400 \mathrm{MHz}, \mathrm{CDCl}_{3}\right) \delta 7.79\left(\mathrm{t}, J=3.1 \mathrm{~Hz}, 1 \mathrm{H},=\mathrm{CHCH}_{2}\right), 7.70(\mathrm{~d}, J=7.4 \mathrm{~Hz}$, 2H, Ar-H), $7.36(\mathrm{~m}, 3 \mathrm{H}, \mathrm{Ar}-\mathrm{H}), 5.84(\mathrm{ddt}, J=16.9,10.3,6.7 \mathrm{~Hz}, 1 \mathrm{H}$, $\mathrm{CH}_{2}=\mathrm{C}_{\underline{H}} \mathrm{CH}_{2} \mathrm{CH}_{2}$ ), $5.03\left(\mathrm{~m}, 2 \mathrm{H}, \underline{\mathrm{CH}}_{2}=\mathrm{CHCH}_{2} \mathrm{CH}_{2}\right.$ ), 2.90 (ddd, $J=19.5,6.7$, $\left.3.1 \mathrm{~Hz}, 1 \mathrm{H},=\mathrm{CHC}_{2}\right), 2.63-2.52(\mathrm{~m}, 1 \mathrm{H}, \mathrm{C} \underline{\mathrm{HCO}}), 2.39(\mathrm{dt}, J=19.5,2.8$ $\left.\mathrm{Hz}, 1 \mathrm{H},=\mathrm{CHC}_{2}\right), 2.20\left(\mathrm{~m}, 2 \mathrm{H}, \mathrm{CH}_{2}=\mathrm{CHC}_{2} \mathrm{CH}_{2}\right), 2.10-1.94(\mathrm{~m}, 1 \mathrm{H}$, $\left.\mathrm{CH}_{2}=\mathrm{CHCH}_{2} \underline{\mathrm{C}}_{2}\right), 1.54\left(\mathrm{~m}, 1 \mathrm{H}, \mathrm{CH}_{2}=\mathrm{CHCH}_{2} \underline{\mathrm{C}}_{2}\right)$.

${ }^{13} \mathrm{C} \mathrm{NMR}\left(101 \mathrm{MHz}, \mathrm{CDCl}_{3}\right) \delta 209.4,157.3,142.8,137.9,131.8,128.4,128.4,127.0$, 115.3, 46.0, 33.2, 31.5, 30.8.

HRMS-ESI $(\mathrm{m} / \mathrm{z})$ : calcd for $\left[\mathrm{C}_{15} \mathrm{H}_{16} \mathrm{O}, \mathrm{M}+\mathrm{Na}\right]^{+}:$235.1093, Found: 235.1096. 
HPLC condition: Chiralpak IC-3 column, $n$-hexane/2-propanol $=99: 1$, flow rate $=1$ $\mathrm{mL} / \mathrm{min}$, wavelength $=254 \mathrm{~nm}, t_{\mathrm{R}}=21.77 \mathrm{~min}$ for major isomer, $t_{\mathrm{R}}=23.49$ min for minor isomer.

\section{5-(2-chloroethyl)-2-phenylcyclopent-2-en-1-one (2bg)}<smiles>O=C1C(c2ccccc2)=CCC1CCCl</smiles>

$2 \mathrm{bg}$

Serial number: cj-3-69, white solid, mp: $57-59{ }^{\circ} \mathrm{C}, 93 \%$ yield $(40.9 \mathrm{mg}), 70 \%$ ee, $[\alpha]_{\mathrm{D}}^{25}=-51.1\left(c 1.3, \mathrm{CHCl}_{3}\right)$, TLC $R_{f}=0.39(\mathrm{PE} / \mathrm{EA}=10: 1, \mathrm{v} / \mathrm{v})$.

$\underline{{ }^{1} \mathrm{H} \mathrm{NMR}}\left(400 \mathrm{MHz}, \mathrm{CDCl}_{3}\right) \delta 7.82\left(\mathrm{~d}, J=3.0 \mathrm{~Hz}, 1 \mathrm{H},=\mathrm{CHCH}_{2}\right), 7.72(\mathrm{~d}, J=7.5 \mathrm{~Hz}$, 2H, Ar-H), 7.39 (m, 3H, Ar-H), $3.88-3.67$ (m, 2H, $\mathrm{CH}_{2} \mathrm{C}_{2} \mathrm{Cl}$ ), 3.01 (ddd, $\left.J=19.4,6.8,3.1 \mathrm{~Hz}, 1 \mathrm{H},=\mathrm{CHC}_{2}\right), 2.79$ (m, 1H, $\left.\underline{\mathrm{HCO}}\right), 2.49-2.35(\mathrm{~m}$, $\left.2 \mathrm{H},=\mathrm{CHC}_{2}, \underline{\mathrm{C}}_{2} \mathrm{CH}_{2} \mathrm{Cl}\right), 1.90\left(\mathrm{~m}, 1 \mathrm{H}, \underline{\mathrm{C}}_{2} \mathrm{CH}_{2} \mathrm{Cl}\right)$.

${ }^{13} \mathrm{C} \mathrm{NMR}\left(101 \mathrm{MHz}, \mathrm{CDCl}_{3}\right) \delta 208.2,157.0,142.6,131.5,128.5,128.5,127.0,44.2$, 43.1, 34.4, 33.2.

HRMS-ESI $(\mathrm{m} / \mathrm{z})$ : calcd for $\left[\mathrm{C}_{13} \mathrm{H}_{13} \mathrm{ClO}, \mathrm{M}+\mathrm{Na}\right]^{+}:$243.0547, Found: 243.0551.

$\underline{\text { HPLC }}$ condition: Chiralpak IC-3 column, $n$-hexane/2-propanol $=98: 2$, flow rate $=1$ $\mathrm{mL} / \mathrm{min}$, wavelength $=254 \mathrm{~nm}, t_{\mathrm{R}}=20.47$ min for major isomer, $t_{\mathrm{R}}=21.54$ min for minor isomer.

\section{5-(2-methoxyethyl)-2-phenylcyclopent-2-en-1-one (2bh)}<smiles>COCCC1CC=C(c2ccccc2)C1=O</smiles>

2bh

Serial number: cj-3-70, colorless oil, 93\% yield $(40.2 \mathrm{mg}), 65 \%$ ee, $[\alpha]_{\mathrm{D}}^{25}=-57.3(c$ $\left.1.1, \mathrm{CHCl}_{3}\right)$, TLC $R_{f}=0.23(\mathrm{PE} / \mathrm{EA}=10: 1, \mathrm{v} / \mathrm{v})$.

$\underline{{ }^{1} \mathrm{H} \text { NMR }}\left(400 \mathrm{MHz}, \mathrm{CDCl}_{3}\right) \delta 7.78\left(\mathrm{t}, J=3.0 \mathrm{~Hz}, 1 \mathrm{H},=\mathrm{CHCH}_{2}\right), 7.74-7.65(\mathrm{~m}, 2 \mathrm{H}$, Ar-H), $7.42-7.29(\mathrm{~m}, 3 \mathrm{H}$, Ar-H), $3.54(\mathrm{td}, J=6.4,1.8 \mathrm{~Hz}, 2 \mathrm{H}$, $\mathrm{CH}_{2} \underline{\mathrm{C}}_{2} \mathrm{OMe}$ ), 3.34 (s, 3H, $\mathrm{CH}_{2} \mathrm{CH}_{2} \mathrm{OMe}$ ), 2.92 (ddd, $J=19.5,6.7,3.1 \mathrm{~Hz}$, 
$\left.1 \mathrm{H},=\mathrm{CHC}_{2}\right), 2.64(\mathrm{~m}, 1 \mathrm{H}, \mathrm{C} \underline{\mathrm{HCO}}), 2.46(\mathrm{dt}, J=19.5,2.8 \mathrm{~Hz}, 1 \mathrm{H}$, $\left.=\mathrm{CHC}_{2}\right), 2.19\left(\mathrm{dtd}, J=13.3,6.5,4.7 \mathrm{~Hz}, 1 \mathrm{H}, \mathrm{C}_{2}{ }_{2} \mathrm{CH}_{2} \mathrm{OMe}\right), 1.69$ (ddt, $J$ $\left.=14.1,9.3,6.2 \mathrm{~Hz}, 1 \mathrm{H}, \mathrm{C}_{2} \mathrm{CH}_{2} \mathrm{OMe}\right)$.

${ }^{13} \mathrm{C} \mathrm{NMR}\left(101 \mathrm{MHz}, \mathrm{CDCl}_{3}\right) \delta 209.1,157.3,142.5,131.8,128.4,128.3,127.0,70.8$, $58.6,44.0,33.4,31.4$.

HRMS-ESI (m/z): calcd for $\left[\mathrm{C}_{14} \mathrm{H}_{16} \mathrm{O}_{2}, \mathrm{M}+\mathrm{Na}\right]^{+}:$239.1043, Found: 239.1045 .

HPLC condition: Chiralpak IC-3 column, $n$-hexane/2-propanol $=95: 5$, flow rate $=1$ $\mathrm{mL} / \mathrm{min}$, wavelength $=254 \mathrm{~nm}, t_{\mathrm{R}}=26.16 \min$ for minor isomer, $t_{\mathrm{R}}=29.41$ min for major isomer.

\section{5-benzyl-2-phenylcyclopent-2-en-1-one (2bi)}<smiles>O=C1C(c2ccccc2)=CCC1Cc1ccccc1</smiles>

2bi

Serial number: cj-3-74, white solid, mp: $79-80{ }^{\circ} \mathrm{C}, 93 \%$ yield $(46.1 \mathrm{mg}), 91 \%$ ee, $[\alpha]_{\mathrm{D}}^{23}=-133.8\left(c 1.1, \mathrm{CHCl}_{3}\right)$, TLC $R_{f}=0.38(\mathrm{PE} / \mathrm{EA}=10: 1, \mathrm{v} / \mathrm{v})$.

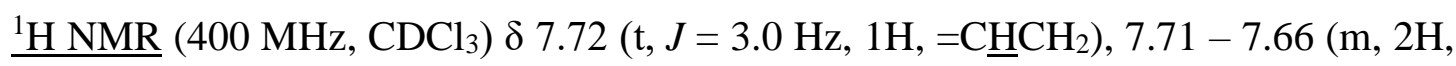
Ar-H), $7.41-7.25$ (m, 5H, Ar-H), 7.22 (d, $J=7.3 \mathrm{~Hz}, 3 \mathrm{H}$, Ar-H), 3.30 (dd, $\left.J=13.8,4.3 \mathrm{~Hz}, 1 \mathrm{H}, \mathrm{PhCH}_{2}\right), 2.92-2.82\left(\mathrm{~m}, 1 \mathrm{H},=\mathrm{CHC} \underline{H}_{2}\right), 2.79-2.63$ (m, 2H, $\left.\mathrm{PhCH}_{2}, \mathrm{C} \underline{\mathrm{HCO}}\right), 2.42\left(\mathrm{dt}, J=19.6,2.7 \mathrm{~Hz}, 1 \mathrm{H},=\mathrm{CHC}_{2}\right)$.

${ }^{13} \mathrm{C} \mathrm{NMR}\left(101 \mathrm{MHz}, \mathrm{CDCl}_{3}\right) \delta 208.6,157.7,142.6,139.4,131.7,129.0,128.5,128.5$, $128.4,127.1,126.4,47.9,37.2,32.5$.

HRMS-ESI $(\mathrm{m} / \mathrm{z})$ : calcd for $\left[\mathrm{C}_{18} \mathrm{H}_{16} \mathrm{O}, \mathrm{M}+\mathrm{Na}\right]^{+}:$271.1093, Found: 271.1098 .

HPLC condition: Chiralpak AD-H column, $n$-hexane/2-propanol $=95: 5$, flow rate $=1$ $\mathrm{mL} / \mathrm{min}$, wavelength $=254 \mathrm{~nm}, t_{\mathrm{R}}=8.68 \min$ for minor isomer, $t_{\mathrm{R}}=9.87$ min for major isomer. 


\section{2,5-diphenylcyclopent-2-en-1-one (2ca)}

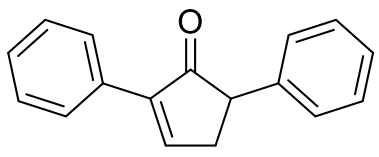

2ca

Serial number: cj-3-27, white solid, mp: $70-72{ }^{\circ} \mathrm{C}, 68 \%$ yield $(31.8 \mathrm{mg}), 84 \%$ ee, $[\alpha]_{\mathrm{D}}^{24}=-85.8\left(c 1, \mathrm{CHCl}_{3}\right)$, TLC $R_{f}=0.32(\mathrm{PE} / \mathrm{EA}=10: 1, \mathrm{v} / \mathrm{v})$.

${ }^{1} \mathrm{H}$ NMR $\left(400 \mathrm{MHz}, \mathrm{CDCl}_{3}\right) \delta 7.99\left(\mathrm{t}, J=3.0 \mathrm{~Hz}, 1 \mathrm{H},=\mathrm{CHCH}_{2}\right), 7.83-7.75(\mathrm{~m}, 2 \mathrm{H}$, Ar-H), $7.47-7.34$ (m, 5H, Ar-H), $7.32-7.21$ (m, 3H, Ar-H), 3.80 (dd, $J=$

7.2, $2.7 \mathrm{~Hz}, 1 \mathrm{H}, \mathrm{C} \underline{\mathrm{HCO}}), 3.30$ (ddd, $J=19.8,7.1,3.1 \mathrm{~Hz}, 1 \mathrm{H},=\mathrm{CHC}_{\mathrm{H}}$ ), $2.87\left(\mathrm{dt}, J=19.8,2.9 \mathrm{~Hz}, 1 \mathrm{H},=\mathrm{CHC}_{2}\right)$.

${ }^{13} \mathrm{C} \mathrm{NMR}\left(101 \mathrm{MHz}, \mathrm{CDCl}_{3}\right) \delta 206.5,157.7,142.4,139.6,131.6,128.9,128.6,128.5$, $127.7,127.1,127.0,52.6,36.1$.

HRMS-ESI $(\mathrm{m} / \mathrm{z})$ : calcd for $\left[\mathrm{C}_{17} \mathrm{H}_{14} \mathrm{O}, \mathrm{M}+\mathrm{Na}\right]^{+}:$257.0937, Found: 257.0940.

$\underline{\text { HPLC }}$ condition: Chiralpak IC-3 column, $n$-hexane/2-propanol $=98: 2$, flow rate $=1$ $\mathrm{mL} / \mathrm{min}$, wavelength $=254 \mathrm{~nm}, t_{\mathrm{R}}=26.67 \min$ for minor isomer, $t_{\mathrm{R}}=28.93$ min for major isomer.

The NMR spectroscopic data were in agreement with those reported in the literature. ${ }^{10}$

\section{2-phenyl-5-(o-tolyl)cyclopent-2-en-1-one (2cb)}

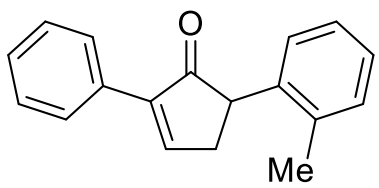

$2 \mathrm{cb}$

Serial number: cj-3-112, colorless oil, $57 \%$ yield $(28.2 \mathrm{mg}), 88 \%$ ee, $[\alpha]_{\mathrm{D}}{ }^{26}=-86.9(c$ $\left.0.35, \mathrm{CHCl}_{3}\right)$, TLC $R_{f}=0.35(\mathrm{PE} / \mathrm{EA}=10: 1, \mathrm{v} / \mathrm{v})$.

${ }^{1} \mathrm{H}$ NMR $\left(400 \mathrm{MHz}, \mathrm{CDCl}_{3}\right) \delta 7.92\left(\mathrm{t}, J=3.0 \mathrm{~Hz}, 1 \mathrm{H},=\mathrm{CHCH}_{2}\right), 7.80-7.74(\mathrm{~m}, 2 \mathrm{H}$, Ar-H), $7.43-7.32$ (m, 3H, Ar-H), 7.22 - 7.10 (m, 3H, Ar-H), $7.02-6.96$ (m, 1H, Ar-H), 3.98 (dd, $J=7.2,2.8 \mathrm{~Hz}, 1 \mathrm{H}, \mathrm{C} \underline{H C O}), 3.26(\mathrm{ddd}, J=19.8$, 7.1, 3.1 Hz, 1H, = $\left.\mathrm{CHC}_{2}\right), 2.71\left(\mathrm{dt}, J=19.8,2.9 \mathrm{~Hz}, 1 \mathrm{H},=\mathrm{CHC}_{2}\right), 2.36$ (s, $\left.3 \mathrm{H}, \mathrm{CH}_{3}\right)$. 
${ }^{13} \mathrm{C} \mathrm{NMR}\left(101 \mathrm{MHz}, \mathrm{CDCl}_{3}\right) \delta 207.3,157.4,142.8,138.5,136.7,131.6,130.7,128.6$, 128.5, 127.1, 127.1, 127.0, 126.4, 49.9, 35.8, 20.0.

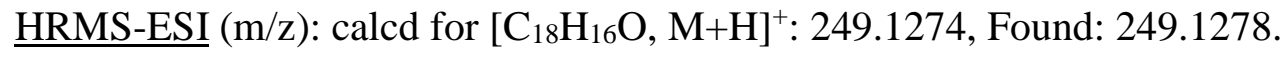

HPLC condition: Chiralpak AD-H column, $n$-hexane/2-propanol $=80: 20$, flow rate $=$ $1 \mathrm{~mL} / \mathrm{min}$, wavelength $=254 \mathrm{~nm}, t_{\mathrm{R}}=6.39 \mathrm{~min}$ for minor isomer, $t_{\mathrm{R}}=8.42$ min for major isomer.

\section{2-phenyl-5-(thiophen-3-yl)cyclopent-2-en-1-one (2cc)}

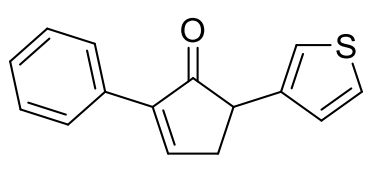

2cc

Serial number: cj-3-61, yellow oil, 47\% yield $(22.5 \mathrm{mg}), 72 \%$ ee, $[\alpha]_{\mathrm{D}}^{23}=-30.1(c 0.2$, $\left.\mathrm{CHCl}_{3}\right)$, TLC $R_{f}=0.31(\mathrm{PE} / \mathrm{EA}=10: 1, \mathrm{v} / \mathrm{v})$.

$\underline{{ }^{1} \mathrm{H} \mathrm{NMR}}\left(400 \mathrm{MHz}, \mathrm{CDCl}_{3}\right) \delta 7.90\left(\mathrm{t}, J=3.0 \mathrm{~Hz}, 1 \mathrm{H},=\mathrm{C}_{\mathbf{H C H}}\right), 7.78-7.68(\mathrm{~m}, 2 \mathrm{H}$, Ar-H), $7.44-7.29$ (m, 4H, Ar-H), 7.18 (d, J=2.9 Hz, 1H, Ar-H), 7.03 (d, $J$ $=5.0 \mathrm{~Hz}, 1 \mathrm{H}, \mathrm{Ar}-\mathrm{H}), 3.90(\mathrm{dd}, J=7.1,2.8 \mathrm{~Hz}, 1 \mathrm{H}, \mathrm{C} \underline{\mathrm{HCO}}), 3.26(\mathrm{ddd}, J=$

19.7, 7.1, 3.1 Hz, 1H, =CHC $\left.\underline{\mathrm{H}}_{2}\right), 2.86\left(\mathrm{dt}, J=19.6,2.9 \mathrm{~Hz}, 1 \mathrm{H},=\mathrm{CHCH}_{2}\right)$.

$\underline{{ }^{13} \mathrm{C} \mathrm{NMR}}\left(101 \mathrm{MHz}, \mathrm{CDCl}_{3}\right) \delta 205.7,157.0,142.2,138.8,131.5,128.6,128.5,127.1$, $126.7,126.2,121.4,47.7,35.1$.

HRMS-ESI (m/z): calcd for $\left[\mathrm{C}_{15} \mathrm{H}_{12} \mathrm{OS}, \mathrm{M}+\mathrm{Na}\right]^{+}:$263.0501, Found: 263.0505 .

$\underline{\text { HPLC }}$ condition: Chiralpak AD-H column, $n$-hexane/2-propanol $=75: 25$, flow rate $=$ $1 \mathrm{~mL} / \mathrm{min}$, wavelength $=254 \mathrm{~nm}, t_{\mathrm{R}}=7.60 \mathrm{~min}$ for minor isomer, $t_{\mathrm{R}}=12.47$ min for major isomer.

\section{2-benzyl-5-methylcyclopent-2-en-1-one (2da)}<smiles>CCCCC(=O)Cc1ccccc1</smiles>

Serial number: cj-2-78, colorless oil, $71 \%$ yield $(26.4 \mathrm{mg}), 84 \%$ ee, $[\alpha]_{\mathrm{D}}^{25}=-25.4(c 1$, $\left.\mathrm{CHCl}_{3}\right), \mathrm{TLC} R_{f}=0.43(\mathrm{PE} / \mathrm{EA}=10: 1, \mathrm{v} / \mathrm{v})$. 
${ }^{1} \mathrm{H}$ NMR $\left(400 \mathrm{MHz}, \mathrm{CDCl}_{3}\right) \delta 7.34-7.15(\mathrm{~m}, 5 \mathrm{H}, \mathrm{Ar}-\mathrm{H}), 7.06\left(\mathrm{~s}, 1 \mathrm{H},=\mathrm{CHCH}_{2}\right)$, $3.48\left(\mathrm{~s}, 2 \mathrm{H}, \mathrm{PhC}_{2}\right), 2.86-2.71\left(\mathrm{~m}, 1 \mathrm{H},=\mathrm{CHC}_{2}\right), 2.42(\mathrm{t}, J=7.5 \mathrm{~Hz}, 1 \mathrm{H}$, C믕), $2.17\left(\mathrm{~s}, 1 \mathrm{H},=\mathrm{CHCH}_{2}\right), 1.19\left(\mathrm{~d}, J=7.5 \mathrm{~Hz}, 3 \mathrm{H}, \underline{\mathrm{CH}}_{3}\right)$.

${ }^{13} \mathrm{C} \mathrm{NMR}\left(101 \mathrm{MHz}, \mathrm{CDCl}_{3}\right) \delta 211.7,157.1,144.8,138.9,129.0,128.5,126.3,40.1$, $35.6,31.5,16.4$.

HRMS-ESI $(\mathrm{m} / \mathrm{z})$ : calcd for $\left[\mathrm{C}_{13} \mathrm{H}_{14} \mathrm{O}, \mathrm{M}+\mathrm{Na}\right]^{+}:$209.0937, Found: 209.0942.

HPLC condition: Chiralpak IC-3 column, $n$-hexane/2-propanol $=99: 1$, flow rate $=1$ $\mathrm{mL} / \mathrm{min}$, wavelength $=254 \mathrm{~nm}, t_{\mathrm{R}}=13.67 \min$ for minor isomer, $t_{\mathrm{R}}=14.51$ min for major isomer.

\section{2-butyl-5-methylcyclopent-2-en-1-one (2db)}<smiles>CCCCC1=CCC(C)C1=O</smiles>

Serial number: cj-1-160, colorless oil, $88 \%$ yield $(26.8 \mathrm{mg}), 90 \%$ ee, $[\alpha]_{\mathrm{D}}{ }^{25}=-37.2(c$ $\left.0.5, \mathrm{CHCl}_{3}\right)$, TLC $R_{f}=0.46(\mathrm{PE} / \mathrm{EA}=10: 1, \mathrm{v} / \mathrm{v})$.

${ }^{1} \mathrm{H}$ NMR $\left(400 \mathrm{MHz}, \mathrm{CDCl}_{3}\right) \delta 7.21-7.17\left(\mathrm{~m}, 1 \mathrm{H},=\mathrm{CHCH}_{2}\right), 2.84-2.73(\mathrm{~m}, 1 \mathrm{H}$, $\left.=\mathrm{CHC}_{2}\right), 2.40-2.30(\mathrm{~m}, 1 \mathrm{H}, \mathrm{C} \underline{\mathrm{HCO}}), 2.18-2.07\left(\mathrm{~m}, 3 \mathrm{H},=\mathrm{CHC}_{2}\right.$, $\left.\mathrm{C}_{2} \mathrm{CH}_{2} \mathrm{CH}_{2} \mathrm{CH}_{3}\right), 1.49-1.37\left(\mathrm{~m}, 2 \mathrm{H}, \mathrm{CH}_{2} \mathrm{C}_{2} \mathrm{CH}_{2} \mathrm{CH}_{3}\right), 1.37-1.25$ (m, $\left.2 \mathrm{H}, \mathrm{CH}_{2} \mathrm{CH}_{2} \mathrm{CH}_{2} \mathrm{CH}_{3}\right), 1.14\left(\mathrm{~d}, J=7.5 \mathrm{~Hz}, 3 \mathrm{H}, \mathrm{CH}_{3}\right), 0.88(\mathrm{t}, J=7.3 \mathrm{~Hz}$, $\left.3 \mathrm{H}, \mathrm{CH}_{2} \mathrm{CH}_{2} \mathrm{CH}_{2} \mathrm{CH}_{3}\right)$.

${ }^{13} \mathrm{C} \mathrm{NMR}\left(101 \mathrm{MHz}, \mathrm{CDCl}_{3}\right) \delta 212.5,155.5,145.3,40.0,35.6,29.9,24.6,22.5,16.5$, 13.9.

HRMS-ESI $(\mathrm{m} / \mathrm{z})$ : calcd for $\left[\mathrm{C}_{10} \mathrm{H}_{16} \mathrm{O}, \mathrm{M}+\mathrm{Na}\right]^{+}:$: 175.1093, Found: 175.1098.

$\underline{\text { HPLC }}$ condition: Chiralpak IC-3 column, $n$-hexane/2-propanol $=99: 1$, flow rate $=0.5$ $\mathrm{mL} / \mathrm{min}$, wavelength $=220 \mathrm{~nm}, t_{\mathrm{R}}=15.41 \mathrm{~min}$ for minor isomer, $t_{\mathrm{R}}=16.34$ min for major isomer. 


\section{2-isopropyl-5-methylcyclopent-2-en-1-one (2dc)}<smiles>CC(C)C1=CCC(C)C1=O</smiles>

Serial number: cj-2-197, colorless oil, 73\% yield $(20.1 \mathrm{mg}), 87 \%$ ee, $[\alpha]_{\mathrm{D}}{ }^{25}=-42.6(c$ $\left.0.1, \mathrm{CHCl}_{3}\right)$, TLC $R_{f}=0.38(\mathrm{PE} / \mathrm{EA}=10: 1, \mathrm{v} / \mathrm{v})$.

$\underline{{ }^{1} \mathrm{H} \mathrm{NMR}}\left(400 \mathrm{MHz}, \mathrm{CDCl}_{3}\right) \delta 7.19\left(\mathrm{~d}, J=1.3 \mathrm{~Hz}, 1 \mathrm{H},=\mathrm{C}_{\mathbf{H C H}}\right), 2.78(\mathrm{~m}, 1 \mathrm{H}$, $\left.=\mathrm{CHC}_{2}\right), 2.59(\mathrm{~m}, 1 \mathrm{H}, \mathrm{C} \underline{\mathrm{HCO}}), 2.36\left(\mathrm{td}, J=7.1,2.4 \mathrm{~Hz}, 1 \mathrm{H},=\mathrm{CHC}_{2}\right)$, $2.11\left(\mathrm{dd}, J=18.7,2.3 \mathrm{~Hz}, 1 \mathrm{H}, \underline{\mathrm{HMe}}_{2}\right), 1.16\left(\mathrm{~d}, J=7.5 \mathrm{~Hz}, 3 \mathrm{H}, \underline{\mathrm{C}}_{3}\right), 1.08$ $\left(\mathrm{d}, J=6.9 \mathrm{~Hz}, 6 \mathrm{H}, \mathrm{CHMe}_{2}\right)$.

${ }^{13} \mathrm{C} \mathrm{NMR}\left(101 \mathrm{MHz}, \mathrm{CDCl}_{3}\right) \delta 212.0,153.5,151.1,40.4,35.3,24.8,21.3,21.2,16.4$. HRMS-EI (m/z): calcd for [ $\left.\mathrm{C}_{9} \mathrm{H}_{14} \mathrm{O}, \mathrm{M}\right]:$ 138.1045, Found: 138.1039.

$\underline{\text { HPLC }}$ condition: Chiralpak IC-3 column, $n$-hexane/2-propanol $=99: 1$, flow rate $=1$ $\mathrm{mL} / \mathrm{min}$, wavelength $=220 \mathrm{~nm}, t_{\mathrm{R}}=8.32 \min$ for minor isomer, $t_{\mathrm{R}}=8.89$ min for major isomer.

\section{3-methyl-[1,1'-bi(cyclopentan)]-5-en-2-one (2dd)}

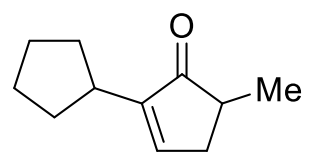

2dd

Serial number: cj-2-36, colorless oil, $92 \%$ yield $(30.2 \mathrm{mg}), 90 \%$ ee, $[\alpha]_{\mathrm{D}}{ }^{25}=-43.1(c 1$, $\left.\mathrm{CHCl}_{3}\right)$, TLC $R_{f}=0.49(\mathrm{PE} / \mathrm{EA}=10: 1, \mathrm{v} / \mathrm{v})$.

$\underline{{ }^{1} \mathrm{H} \mathrm{NMR}}\left(400 \mathrm{MHz}, \mathrm{CDCl}_{3}\right) \delta 7.19\left(\mathrm{~s}, 1 \mathrm{H},=\mathrm{CHCH}_{2}\right), 2.88-2.75\left(\mathrm{~m}, 1 \mathrm{H},=\mathrm{CHC}_{2}\right)$, $2.75-2.63(\mathrm{~m}, 1 \mathrm{H}, \mathrm{C} \underline{\mathrm{HCO}}), 2.37\left(\mathrm{~m}, 1 \mathrm{H},=\mathrm{CHC}_{2}\right), 2.13(\mathrm{~m}, 1 \mathrm{H}$, $\left.\mathrm{C} \underline{\mathrm{H}}\left(\mathrm{CH}_{2}\right)_{4}\right), 2.00-1.86\left(\mathrm{~m}, 2 \mathrm{H}, \mathrm{CH}\left(\mathrm{C}_{2}\right)_{4}\right), 1.75-1.56\left(\mathrm{~m}, 4 \mathrm{H}, \mathrm{CH}\left(\underline{\mathrm{CH}}_{2}\right)_{4}\right)$, $1.47-1.32\left(\mathrm{~m}, 2 \mathrm{H}, \mathrm{CH}\left(\mathrm{C}_{2}\right)_{4}\right), 1.17\left(\mathrm{~d}, J=7.5 \mathrm{~Hz}, 3 \mathrm{H}, \underline{\mathrm{C}}_{3}\right)$.

${ }^{13} \mathrm{C} \mathrm{NMR}\left(101 \mathrm{MHz}, \mathrm{CDCl}_{3}\right) \delta 212.3,153.5,149.0,40.3,36.1,35.4,31.7,31.6,25.1$, $25.0,16.4$.

HRMS-ESI $(\mathrm{m} / \mathrm{z})$ : calcd for $\left[\mathrm{C}_{11} \mathrm{H}_{16} \mathrm{O}, \mathrm{M}+\mathrm{Na}\right]^{+}:$187.1093, Found: 187.1096. 
HPLC condition: Chiralpak IC-3 column, $n$-hexane/2-propanol $=98: 2$, flow rate $=1$ $\mathrm{mL} / \mathrm{min}$, wavelength $=220 \mathrm{~nm}, t_{\mathrm{R}}=7.84$ min for minor isomer, $t_{\mathrm{R}}=8.60$ min for major isomer.

\section{2-cyclohexyl-5-methylcyclopent-2-en-1-one (2de)}

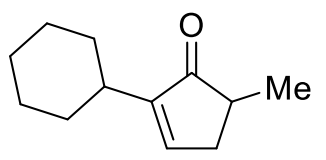

2de

Serial number: cj-1-159, colorless oil, $86 \%$ yield $(30.5 \mathrm{mg}), 91 \%$ ee, $[\alpha]_{\mathrm{D}}^{25}=-45.4(c$ $\left.1, \mathrm{CHCl}_{3}\right)$, TLC $R_{f}=0.52(\mathrm{PE} / \mathrm{EA}=10: 1, \mathrm{v} / \mathrm{v})$.

$\underline{{ }^{1} \mathrm{H} \text { NMR }}\left(400 \mathrm{MHz}, \mathrm{CDCl}_{3}\right) \delta 7.16\left(\mathrm{~m}, 1 \mathrm{H},=\mathrm{CHCH}_{2}\right), 2.78\left(\mathrm{~m}, 1 \mathrm{H},=\mathrm{CHC}_{2}\right), 2.39$ - $2.21\left(\mathrm{~m}, 2 \mathrm{H}, \mathrm{C} \underline{\mathrm{HCO}},=\mathrm{CHC}_{2}\right), 2.11(\mathrm{dtd}, J=18.7,2.5,1.6 \mathrm{~Hz}, 1 \mathrm{H}$, $\left.\mathrm{C} \underline{\mathrm{H}}\left(\mathrm{CH}_{2}\right)_{5}\right), 1.87-1.67\left(\mathrm{~m}, 5 \mathrm{H}, \mathrm{CH}\left(\mathrm{C}_{2}\right)_{5}\right), 1.41-1.27$ (m, 2H, $\left.\mathrm{CH}\left(\mathrm{C}_{2}\right)_{5}\right)$, $1.25-1.05$ (m, 6H, $\left.\underline{\mathrm{CH}}_{3}, \mathrm{CH}\left(\mathrm{C}_{2}\right)_{5}\right)$.

${ }^{13} \mathrm{C} \mathrm{NMR}\left(101 \mathrm{MHz}, \mathrm{CDCl}_{3}\right) \delta 212.0,153.9,150.2,40.3,35.5,34.3,32.0,31.9,26.4$, $26.3,26.2,16.5$.

HRMS-ESI $(\mathrm{m} / \mathrm{z})$ : calcd for $\left[\mathrm{C}_{12} \mathrm{H}_{18} \mathrm{O}, \mathrm{M}+\mathrm{Na}\right]^{+}:$201.1250, Found: 201.1255.

$\underline{\text { HPLC }}$ condition: Chiralpak IC-3 column, $n$-hexane/2-propanol $=98: 2$, flow rate $=1$ $\mathrm{mL} / \mathrm{min}$, wavelength $=220 \mathrm{~nm}, t_{\mathrm{R}}=7.14$ min for minor isomer, $t_{\mathrm{R}}=7.90$ min for major isomer.

2-(cyclohex-1-en-1-yl)-5-methylcyclopent-2-en-1-one (2ea)

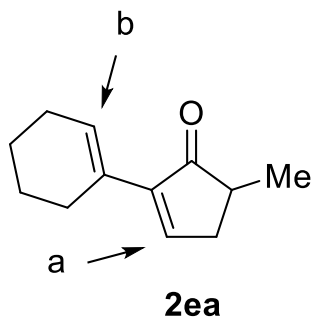

Serial number: cj-2-68, colorless oil, $92 \%$ yield $(32.4 \mathrm{mg}), 94 \%$ ee, $[\alpha]_{\mathrm{D}}{ }^{25}=-80.4(c 1$, $\left.\mathrm{CHCl}_{3}\right), \mathrm{TLC} R_{f}=0.44(\mathrm{PE} / \mathrm{EA}=10: 1, \mathrm{v} / \mathrm{v})$. 
$\underline{{ }^{1} \mathrm{H} \mathrm{NMR}}\left(400 \mathrm{MHz}, \mathrm{CDCl}_{3}\right) \delta 7.30\left(\mathrm{~s}, 1 \mathrm{H}, \mathrm{H}_{\mathrm{a}}\right), 6.93\left(\mathrm{~s}, 1 \mathrm{H}, \mathrm{H}_{\mathrm{b}}\right), 2.87-2.79(\mathrm{~m}, 1 \mathrm{H}$, $\left.=\mathrm{CH}_{\mathrm{a}} \underline{\mathrm{H}}_{2}\right), 2.44(\mathrm{~m}, 1 \mathrm{H}, \mathrm{C} \underline{\mathrm{HCO}}), 2.22-2.14\left(\mathrm{~m}, 5 \mathrm{H},=\mathrm{CH}_{\mathrm{a}} \underline{\mathrm{C}}_{2},=\mathrm{CH}_{\mathrm{b}} \underline{\mathrm{H}}_{2}\right.$, $\left.=\mathrm{CH}_{\mathrm{b}}\left(\mathrm{CH}_{2}\right)_{3} \underline{\mathrm{CH}}_{2}\right), 1.71\left(\mathrm{q}, J=5.8 \mathrm{~Hz}, 2 \mathrm{H},=\mathrm{CH}_{\mathrm{b}} \mathrm{CH}_{2}\left(\mathrm{C}_{2}\right)_{2} \mathrm{CH}_{2}\right), 1.62$ (q, $J$ $\left.=5.7 \mathrm{~Hz}, 2 \mathrm{H},=\mathrm{CH}_{\mathrm{b}} \mathrm{CH}_{2}\left(\underline{\mathrm{C}}_{2}\right)_{2} \mathrm{CH}_{2}\right), 1.20\left(\mathrm{~d}, J=7.4 \mathrm{~Hz}, 3 \mathrm{H}, \mathrm{C}_{3}\right)$.

${ }^{13} \mathrm{C} \mathrm{NMR}\left(101 \mathrm{MHz}, \mathrm{CDCl}_{3}\right) \delta 211.0,153.2,142.0,128.6,128.6,41.4,34.7,26.6$, 25.6, 22.6, 22.0, 16.6.

HRMS-ESI $(\mathrm{m} / \mathrm{z})$ : calcd for $\left[\mathrm{C}_{12} \mathrm{H}_{16} \mathrm{O}, \mathrm{M}+\mathrm{Na}\right]^{+}:$199.1093, Found: 199.1098.

$\underline{\text { HPLC }}$ condition: Chiralpak IC-3 column, $n$-hexane/2-propanol $=99: 1$, flow rate $=1$ $\mathrm{mL} / \mathrm{min}$, wavelength $=220 \mathrm{~nm}, t_{\mathrm{R}}=15.65 \min$ for minor isomer, $t_{\mathrm{R}}=17.94$ min for major isomer.

\section{5-methyl-2-(oct-1-yn-1-yl)cyclopent-2-en-1-one (2fa)}

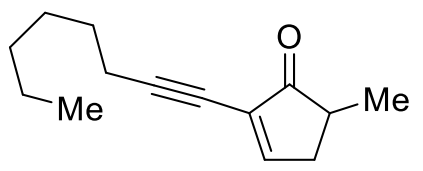

$2 \mathrm{fa}$

Serial number: cj-2-74, colorless oil, 51\% yield $(20.8 \mathrm{mg}), 93 \%$ ee, $[\alpha]_{\mathrm{D}}{ }^{25}=-67.2(c 1$, $\left.\mathrm{CHCl}_{3}\right)$, TLC $R_{f}=0.57(\mathrm{PE} / \mathrm{EA}=10: 1, \mathrm{v} / \mathrm{v})$.

$\underline{{ }^{1} \mathrm{H} \mathrm{NMR}}\left(400 \mathrm{MHz}, \mathrm{CDCl}_{3}\right) \delta 7.61\left(\mathrm{t}, J=3.1 \mathrm{~Hz}, 1 \mathrm{H},=\mathrm{CHCH}_{2}\right), 2.92(\mathrm{~m}, 1 \mathrm{H}$, $\left.=\mathrm{CHC}_{2}\right), 2.48-2.35\left(\mathrm{~m}, 3 \mathrm{H}, \mathrm{C} \underline{\mathrm{HCO}},=\mathrm{CHC}_{2} 2, \equiv \mathrm{CC}_{2}\right), 2.25(\mathrm{dt}, J=$ 19.9, $\left.2.7 \mathrm{~Hz}, 1 \mathrm{H}, \equiv \mathrm{CC}_{2}\right), 1.61-1.51\left(\mathrm{~m}, 2 \mathrm{H}, \equiv \mathrm{CCH}_{2} \underline{\mathrm{C}}_{2}\right), 1.44-1.35(\mathrm{~m}$, $\left.2 \mathrm{H}, \equiv \mathrm{C}\left(\mathrm{CH}_{2}\right)_{2} \underline{\mathrm{C}}_{2}\right), 1.33-1.25\left(\mathrm{~m}, 4 \mathrm{H}, \equiv \mathrm{C}\left(\mathrm{CH}_{2}\right)_{3}\left(\mathrm{C}_{2}\right)_{2}\right), 1.19(\mathrm{~d}, J=7.6$ $\left.\mathrm{Hz}, 3 \mathrm{H}, \underline{\mathrm{C}}_{3}\right), 0.88$ (t, $\left.J=6.9 \mathrm{~Hz}, 3 \mathrm{H}, \equiv \mathrm{C}\left(\mathrm{CH}_{2}\right)_{5} \mathrm{C}_{3}\right)$.

${ }^{13} \mathrm{C} \mathrm{NMR}\left(101 \mathrm{MHz}, \mathrm{CDCl}_{3}\right) \delta 208.9,162.4,129.3,97.3,71.4,39.4,36.0,31.3,28.6$, $28.5,22.5,19.6,16.4,14.1$.

HRMS-ESI $(\mathrm{m} / \mathrm{z})$ : calcd for $\left[\mathrm{C}_{14} \mathrm{H}_{20} \mathrm{O}, \mathrm{M}+\mathrm{Na}\right]^{+}:$227.1406, Found: 227.1410.

$\underline{\text { HPLC }}$ condition: Chiralpak IC-3 column, $n$-hexane/2-propanol $=99: 1$, flow rate $=1$ $\mathrm{mL} / \mathrm{min}$, wavelength $=210 \mathrm{~nm}, t_{\mathrm{R}}=25.90 \mathrm{~min}$ for minor isomer, $t_{\mathrm{R}}=27.83$ min for major isomer. 


\section{4,5-dimethyl-2-phenylcyclopent-2-en-1-one (2ga)}<smiles>CC1C=C(c2ccccc2)C(=O)C1C</smiles>

Serial number: cj-2-64, colorless oil, 94\% yield (35.1 mg), dr =1.1:1 (mixture), 97\%, $92 \%$ ee, $[\alpha]_{\mathrm{D}}{ }^{25}=-38.8\left(c 1, \mathrm{CHCl}_{3}\right)$, TLC $R_{f}=0.44,0.45(\mathrm{PE} / \mathrm{EA}=10: 1, \mathrm{v} / \mathrm{v})$.

${ }^{1} \mathrm{H} \mathrm{NMR}\left(400 \mathrm{MHz}, \mathrm{CDCl}_{3}\right) \delta 7.72(\mathrm{~m}, 5 \mathrm{H}, \mathrm{Ar}-\mathrm{H}), 7.63(\mathrm{~d}, J=2.6 \mathrm{~Hz}, 1 \mathrm{H}$, $=\mathrm{C} \underline{\mathrm{HCHMe}}), 7.36(\mathrm{~m}, 6 \mathrm{H},=\mathrm{C} \underline{\mathrm{HCHMe}}, \mathrm{Ar}-\mathrm{H}), 3.11(\mathrm{td}, J=7.1,3.0 \mathrm{~Hz}, 1 \mathrm{H}$, $=\mathrm{CHC} \underline{\mathrm{HMe}}), 2.69(\mathrm{p}, J=7.4 \mathrm{~Hz}, 1 \mathrm{H},=\mathrm{CHC} \underline{\mathrm{HMe}}), 2.55(\mathrm{dt}, J=7.4,2.9 \mathrm{~Hz}$, 1H, CㅂCO), 2.10 (dd, $J=7.4,2.8 \mathrm{~Hz}, 1 \mathrm{H}, \mathrm{C} \underline{\mathrm{HCO}}), 1.28(\mathrm{t}, J=7.9 \mathrm{~Hz}, 7 \mathrm{H}$, $\mathrm{C}_{3} \mathrm{CHCO}$ ), 1.15 (dd, $J=14.9,7.5 \mathrm{~Hz}, 6 \mathrm{H}, \mathrm{C}_{3} \mathrm{CHCO}$ ).

${ }^{13} \mathrm{C} \mathrm{NMR}\left(101 \mathrm{MHz}, \mathrm{CDCl}_{3}\right) \delta 210.1,209.6,162.8,161.6,141.3,140.8,131.7,131.6$, $128.4,128.4,128.4,127.1,127.1,50.3,45.1,42.0,36.8,19.0,15.7,14.5$, 11.4 .

HRMS-ESI $(\mathrm{m} / \mathrm{z})$ : calcd for $\left[\mathrm{C}_{13} \mathrm{H}_{14} \mathrm{O}, \mathrm{M}+\mathrm{Na}\right]^{+}:$209.0937, Found: 209.0942.

HPLC condition: Chiralpak IC-3 column, $n$-hexane/2-propanol $=99: 1$, flow rate $=1$ $\mathrm{mL} / \mathrm{min}$, wavelength $=254 \mathrm{~nm}, t_{\mathrm{R}}=16.45$ min for major isomer, $t_{\mathrm{R}}=18.39$ $\min$ for minor isomer; $t_{\mathrm{R}}=20.42 \mathrm{~min}$ for major isomer, $t_{\mathrm{R}}=21.51 \mathrm{~min}$ for minor isomer.

\section{5-isopropyl-4-methyl-2-phenylcyclopent-2-en-1-one (2gb)}<smiles>CC(C)C1C(=O)C(c2ccccc2)=CC1[18OH]</smiles>

Serial number: cj-4-125, colorless oil, $88 \%$ yield (37.6 mg), $\mathrm{dr}=1.4: 1$ (mixture), $73 \%$, $97 \%$ ee, $[\alpha]_{\mathrm{D}}^{25}=-12.3\left(c 1, \mathrm{CHCl}_{3}\right)$, TLC $R_{f}=0.48,0.49(\mathrm{PE} / \mathrm{EA}=10: 1, \mathrm{v} / \mathrm{v})$.

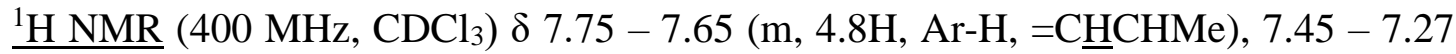
$(\mathrm{m}, 4.8 \mathrm{H}, \mathrm{Ar}-\mathrm{H}), 3.12(\mathrm{td}, J=7.2,2.7 \mathrm{~Hz}, 0.7 \mathrm{H},=\mathrm{CHC} \underline{\mathrm{HMe}}), 2.78(\mathrm{dt}, J=$

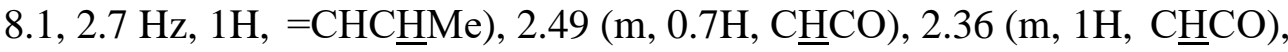


$2.09\left(\mathrm{~m}, 1.7 \mathrm{H}, \mathrm{CHMe}_{2}\right), 1.26(\mathrm{~m}, 5.8 \mathrm{H},=\mathrm{CHCHMe}), 1.18(\mathrm{~m}, 2.1 \mathrm{H}$,

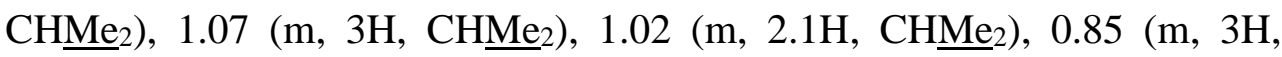
$\left.\mathrm{CHMe}_{2}\right)$.

${ }^{13} \mathrm{C}$ NMR $\left(101 \mathrm{MHz}, \mathrm{CDCl}_{3}\right) \delta$ 209.3, 208.8, 163.1, 161.9, 142.2, 141.9, 131.7, 131.6, $128.4,128.3,128.2,127.1,110.7,60.8,56.3,36.8,35.5,29.2,27.2,22.3$, $20.8,20.6,19.6,17.8,15.5$.

HRMS-ESI $(\mathrm{m} / \mathrm{z})$ : calcd for $\left[\mathrm{C}_{15} \mathrm{H}_{18} \mathrm{O}, \mathrm{M}+\mathrm{Na}\right]^{+}:$237.1250, Found: 237.1252.

$\underline{\text { HPLC }}$ condition: Chiralpak OJ-3 column, $n$-hexane/2-propanol $=98: 2$, flow rate $=1$ $\mathrm{mL} / \mathrm{min}$, wavelength $=254 \mathrm{~nm}, t_{\mathrm{R}}=9.08 \mathrm{~min}$ for minor isomer, $t_{\mathrm{R}}=17.16$ $\min$ for major isomer; $t_{\mathrm{R}}=15.66 \mathrm{~min}$ for minor isomer, $t_{\mathrm{R}}=25.73 \mathrm{~min}$ for major isomer.

\section{5-methyl-2,4-diphenylcyclopent-2-en-1-one (2gc)}<smiles>CC1C(=O)C(c2ccccc2)=CC1c1ccccc1</smiles>

Serial number: cj-4-126, yellow oil, $94 \%$ yield (46.6 mg), $\mathrm{dr}=2: 1$ (mixture), 56\%, 94\% ee, $[\alpha]_{\mathrm{D}}^{25}=-9.6\left(c 1, \mathrm{CHCl}_{3}\right)$, TLC $R_{f}=0.42,0.44(\mathrm{PE} / \mathrm{EA}=10: 1, \mathrm{v} / \mathrm{v})$.

${ }^{1} \mathrm{H}$ NMR $\left(400 \mathrm{MHz}, \mathrm{CDCl}_{3}\right) \delta 7.87-7.82(\mathrm{~m}, 3.5 \mathrm{H}, \mathrm{Ar}-\mathrm{H},=\mathrm{C} \underline{\mathrm{HCHPh}}), 7.58-7.03$ (m, 11H, Ar-H), $7.15-7.09$ (m, 2H, Ar-H), $4.37(\mathrm{dd}, J=7.0,3.0 \mathrm{~Hz}, 1 \mathrm{H}$, $=\mathrm{CHC} \underline{H P h}), 3.72(\mathrm{~s}, 0.5 \mathrm{H},=\mathrm{CHC} \underline{H P h}), 3.00(\mathrm{~m}, 1 \mathrm{H}, \underline{\mathrm{CHCO}}), 2.53(\mathrm{~m}$, $0.5 \mathrm{H}, \underline{\mathrm{CHCO}}), 1.42(\mathrm{~d}, J=7.4 \mathrm{~Hz}, 1.5 \mathrm{H}, \underline{\mathrm{Me}}), 0.85(\mathrm{~d}, J=7.6 \mathrm{~Hz}, 3 \mathrm{H}, \underline{\mathrm{Me}})$. ${ }^{13} \mathrm{C}$ NMR $\left(101 \mathrm{MHz}, \mathrm{CDCl}_{3}\right) \delta 209.7,209.1,158.4,158.3,142.4,142.0,141.8,138.7$, $131.5,131.4,129.0,128.7,128.6,127.4,127.3,53.0,52.3,48.7,46.3,14.6$, 13.1.

HRMS-ESI $(\mathrm{m} / \mathrm{z})$ : calcd for $\left[\mathrm{C}_{18} \mathrm{H}_{16} \mathrm{O}, \mathrm{M}+\mathrm{Na}\right]^{+}:$271.1093, Found: 271.1092.

$\underline{\text { HPLC }}$ condition: Chiralpak IC-3 column, $n$-hexane/2-propanol $=98: 2$, flow rate $=1$ $\mathrm{mL} / \mathrm{min}$, wavelength $=254 \mathrm{~nm}, t_{\mathrm{R}}=12.41$ min for major isomer, $t_{\mathrm{R}}=16.39$ $\min$ for minor isomer; $t_{\mathrm{R}}=14.85 \mathrm{~min}$ for minor isomer, $t_{\mathrm{R}}=18.01 \mathrm{~min}$ for major isomer. 


\section{5-methyl-2,3-diphenylcyclopent-2-en-1-one (2ha)}

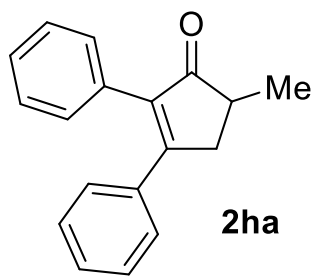

Serial number: cj-2-14, colorless oil, $71 \%$ yield $(35.0 \mathrm{mg}), 94 \%$ ee, $[\alpha]_{\mathrm{D}}{ }^{24}=-114.6(c$ $\left.1, \mathrm{CHCl}_{3}\right)$, TLC $R_{f}=0.37(\mathrm{PE} / \mathrm{EA}=10: 1, \mathrm{v} / \mathrm{v})$.

${ }^{1} \mathrm{H}$ NMR $\left(400 \mathrm{MHz}, \mathrm{CDCl}_{3}\right) \delta 7.53-7.10(\mathrm{~m}, 10 \mathrm{H}, \mathrm{Ar}-\mathrm{H}), 3.29(\mathrm{dd}, J=18.0,6.8 \mathrm{~Hz}$, $\left.1 \mathrm{H}, \underline{\mathrm{C}}_{2}\right), 2.76-2.50\left(\mathrm{~m}, 2 \mathrm{H}, \mathrm{CHCO}, \underline{\mathrm{CH}}_{2}\right), 1.35$ (d, J=7.2 Hz, 3H, $\left.\underline{\mathrm{CH}}_{3}\right)$.

${ }^{13} \mathrm{C} \mathrm{NMR}\left(101 \mathrm{MHz}, \mathrm{CDCl}_{3}\right) \delta 210.0,166.1,138.6,135.8,132.5,129.8,129.5,128.4$, $128.4,128.1,127.8,40.2,38.7,16.8$.

HRMS-ESI $(\mathrm{m} / \mathrm{z})$ : calcd for $\left[\mathrm{C}_{18} \mathrm{H}_{16} \mathrm{O}, \mathrm{M}+\mathrm{Na}\right]^{+}:$271.1093, Found: 271.1098.

$\underline{\text { HPLC }}$ condition: Chiralpak IC-3 column, $n$-hexane/2-propanol $=98: 2$, flow rate $=1$ $\mathrm{mL} / \mathrm{min}$, wavelength $=254 \mathrm{~nm}, t_{\mathrm{R}}=25.06$ min for minor isomer, $t_{\mathrm{R}}=26.98$ min for major isomer.

\section{4,4,5-trimethyl-2-phenylcyclopent-2-en-1-one (2ia)}

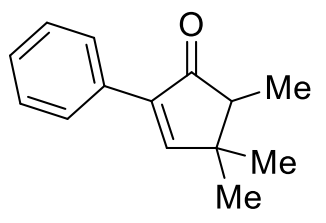

2ia

Serial number: cj-2-21, colorless oil, $88 \%$ yield $(35.0 \mathrm{mg}), 98 \%$ ee, $[\alpha]_{\mathrm{D}}^{25}=-142.4(c$ $\left.1, \mathrm{CHCl}_{3}\right)$, TLC $R_{f}=0.46(\mathrm{PE} / \mathrm{EA}=10: 1, \mathrm{v} / \mathrm{v})$.

$\underline{{ }^{1} \mathrm{H} \text { NMR }}\left(400 \mathrm{MHz}, \mathrm{CDCl}_{3}\right) \delta 7.80-7.69(\mathrm{~m}, 2 \mathrm{H}, \mathrm{Ar}-\mathrm{H}), 7.59(\mathrm{~s}, 1 \mathrm{H},=\mathrm{C} \underline{\mathrm{H}}), 7.43-$ $7.31(\mathrm{~m}, 3 \mathrm{H}, \mathrm{Ar}-\mathrm{H}), 2.36(\mathrm{q}, J=7.4 \mathrm{~Hz}, 1 \mathrm{H}, \mathrm{C} \underline{\mathrm{HCO}}), 1.31\left(\mathrm{~s}, 3 \mathrm{H}, \underline{\mathrm{C}}_{3}\right)$, $1.19\left(\mathrm{~d}, J=7.4 \mathrm{~Hz}, 3 \mathrm{H}, \underline{\mathrm{C}}_{3} \mathrm{CHCO}\right), 1.12\left(\mathrm{~s}, 3 \mathrm{H}, \underline{\mathrm{C}}_{3}\right)$.

${ }^{13} \mathrm{C} \mathrm{NMR}\left(101 \mathrm{MHz}, \mathrm{CDCl}_{3}\right) \delta 209.2,166.1,139.2,131.6,128.4,128.3,127.1,53.4$, 41.5, 27.7, 24.4, 10.2.

HRMS-ESI $(\mathrm{m} / \mathrm{z})$ : calcd for $\left[\mathrm{C}_{14} \mathrm{H}_{16} \mathrm{O}, \mathrm{M}+\mathrm{Na}\right]^{+}:$223.1093, Found: 223.1098. 
$\underline{\text { HPLC }}$ condition: Chiralpak IC-3 column, $n$-hexane/2-propanol $=98: 2$, flow rate $=1$

$\mathrm{mL} / \mathrm{min}$, wavelength $=254 \mathrm{~nm}, t_{\mathrm{R}}=9.21 \min$ for major isomer, $t_{\mathrm{R}}=9.89$ min for minor isomer. 


\section{X-Ray Diffraction Analysis of $(R)-2 a a$}

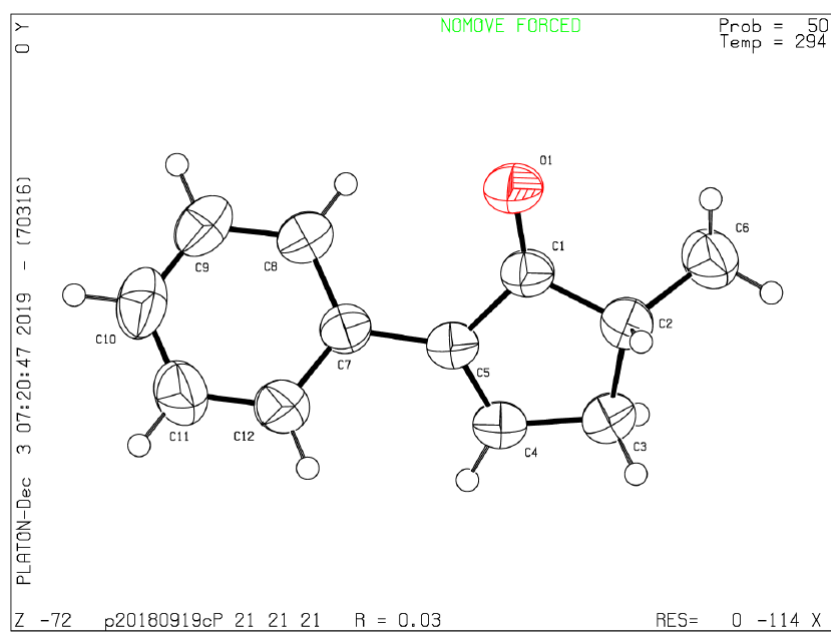

Identification code

Empirical formula

Formula weight

Temperature/K

Crystal system

Space group

$$
\begin{aligned}
& \mathrm{a} / \AA \\
& \mathrm{b} / \AA \\
& \mathrm{c} / \AA \\
& \alpha /{ }^{\circ} \\
& \beta /{ }^{\circ} \\
& \gamma /{ }^{\circ}
\end{aligned}
$$

Volume $/ \AA^{3}$

$\mathrm{Z}$

$\rho_{\text {calc }} \mathrm{g} / \mathrm{cm}^{3}$

$\mu / \mathrm{mm}^{-1}$

$\mathrm{F}(000)$

Crystal size $/ \mathrm{mm}^{3}$

Radiation

$2 \Theta$ range for data collection/ ${ }^{\circ}$

Index ranges

Reflections collected

Independent reflections

Data/restraints/parameters

Goodness-of-fit on $\mathrm{F}^{2}$

Final $R$ indexes $[\mathrm{I}>=2 \sigma(\mathrm{I})]$

Final $\mathrm{R}$ indexes [all data]

Largest diff. peak/hole / e $\AA^{-3}$
P20180919c

$\mathrm{C}_{12} \mathrm{H}_{12} \mathrm{O}$

172.22

294.15

orthorhombic

$\mathrm{P} 2{ }_{1} 2_{1} 2_{1}$

$6.52802(8)$

$7.91878(6)$

$18.51328(16)$

90

90

90

$957.025(15)$

4

1.195

0.581

368.0

$0.36 \times 0.24 \times 0.22$

$\mathrm{CuK \alpha}(\lambda=1.54184)$

9.554 to 158.602

$-8 \leq \mathrm{h} \leq 7,-9 \leq \mathrm{k} \leq 10,-23 \leq 1 \leq 21$

12882

$2021\left[R_{\text {int }}=0.0224, R_{\text {sigma }}=0.0105\right]$

2021/0/120

1.067

$\mathrm{R}_{1}=0.0310, \mathrm{wR}_{2}=0.0820$

$\mathrm{R}_{1}=0.0315, \mathrm{wR}_{2}=0.0825$

$0.12 /-0.09$ 


\section{Transformations of Cyclization Product 2aa}

\subsection{Transformation of 2 aa to 5}

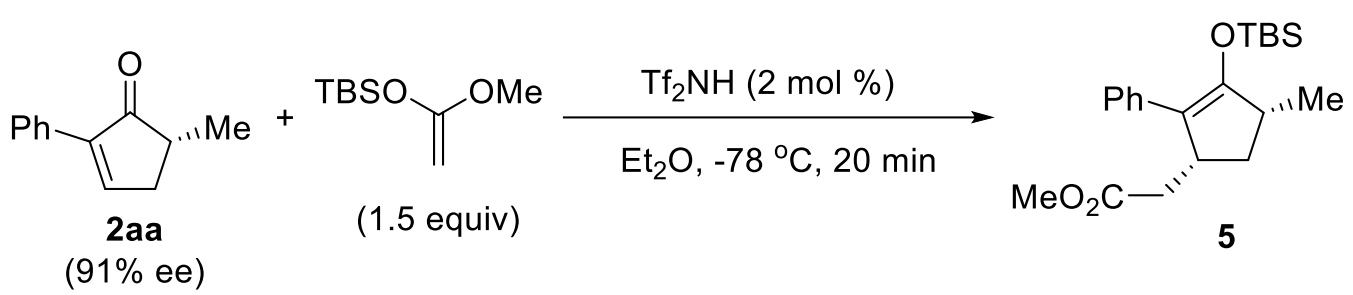

To a solution of $2 \mathbf{a a}(34.4 \mathrm{mg}, 0.2 \mathrm{mmol})$ in dry $\mathrm{Et}_{2} \mathrm{O}$ was added silyl ketene acetal (56.4 mg, $0.3 \mathrm{mmol}, 1.5$ equiv) under Ar. After cooling the solution to $-78{ }^{\circ} \mathrm{C}$, $\mathrm{Tf}_{2} \mathrm{NH}\left(0.1 \mathrm{M}\right.$ in $\left.\mathrm{Et}_{2} \mathrm{O}, 40 \mu \mathrm{L}, 2 \mathrm{~mol} \%\right)$ was added. The mixture stirred for $10 \mathrm{~min}$ at this temperature and was then quenched with $\mathrm{MeOH}(1 \mathrm{~mL})$, and concentrated to afford a colorless residue, which was purified by column on silica-gel to afford $\mathbf{5}$ as a colorless oil (60.0 mg, 84\% yield, d.r. $=10: 1,91 \%$ ee). Serial number: cj-2-152, TLC $R_{f}=0.60(\mathrm{PE} / \mathrm{EA}=10: 1, \mathrm{v} / \mathrm{v}) ;[\alpha]_{\mathrm{D}}^{25}=14.0\left(c 0.5, \mathrm{CHCl}_{3}\right)$.

${ }^{1} \mathrm{H}$ NMR $\left(400 \mathrm{MHz}, \mathrm{CDCl}_{3}\right) \delta 7.34-7.29$ (m, 2H, Ar-H), 7.25 - 7.19 (m, 2H, Ar-H), $7.11-7.05(\mathrm{~m}, 1 \mathrm{H}, \operatorname{Ar}-\mathrm{H}), 3.55(\mathrm{~s}, 3 \mathrm{H}, \mathrm{COOMe}), 3.48-3.38(\mathrm{~m}, 1 \mathrm{H}$, $\mathrm{C}_{2} \mathrm{COOMe}$ ), 2.64 - 2.53 (m, 2H, CㅍMe, $\underline{\mathrm{C}}_{2} \mathrm{COOMe}$ ), 2.44 (dt, $J=13.1$, $8.7 \mathrm{~Hz}, 1 \mathrm{H}, \mathrm{C}_{\mathbf{H C H}} \mathrm{COOMe}, 1.96\left(\mathrm{dd}, J=15.4,10.8 \mathrm{~Hz}, 1 \mathrm{H}, \underline{\mathrm{C}}_{2}\right), 1.12$ (d, $\left.J=7.0 \mathrm{~Hz}, 4 \mathrm{H}, \underline{\mathrm{C}}_{2}, \underline{\mathrm{C}}_{3}\right), 0.79$ (d, $\left.J=0.8 \mathrm{~Hz}, 9 \mathrm{H}, \mathrm{Si}^{t} \underline{\mathrm{BuMe}}_{2}\right),-0.06$ (s, $\left.3 \mathrm{H}, \mathrm{Si}^{t} \mathrm{BuMe}_{2}\right),-0.31$ (s, 3H, $\left.\mathrm{Si}^{t} \mathrm{BuMe}_{2}\right)$.

${ }^{13} \mathrm{C} \mathrm{NMR}\left(101 \mathrm{MHz}, \mathrm{CDCl}_{3}\right) \delta 173.8,154.2,135.5,128.2,128.0,125.8,118.3,51.4$, $40.9,40.0,38.6,35.3,25.6,20.4,18.1,-4.1,-4.4$.

HRMS-EI (m/z): calcd for $\left[\mathrm{C}_{21} \mathrm{H}_{32} \mathrm{O}_{3} \mathrm{Si}, \mathrm{M}\right]^{+}:$360.2121, Found: 360.2114 .

HPLC conditions: Chiralpak IF-3 column, $n$-hexane/2-propanol $=99: 1$, flow rate $=1$ $\mathrm{mL} / \mathrm{min}$, wavelength $=254 \mathrm{~nm}, t_{\mathrm{R}}=6.05 \min$ for minor isomer, $t_{\mathrm{R}}=6.49$ $\min$ for major isomer. 


\subsection{Transformation of 2aa to 6}

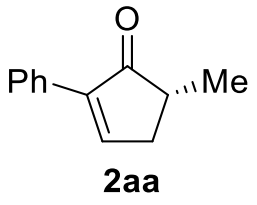

$91 \%$ ee

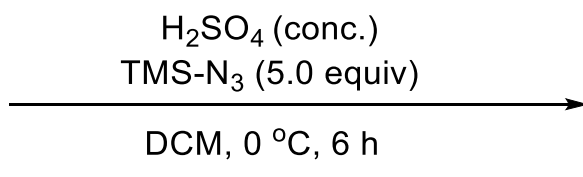

$\mathrm{DCM}, 0^{\circ} \mathrm{C}, 6 \mathrm{~h}$

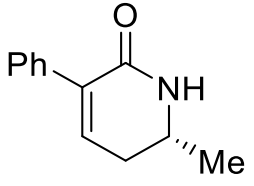

6

To a mixture of $2 \mathbf{a a}(34.4 \mathrm{mg}, 0.2 \mathrm{mmol})$ in $\mathrm{DCM}(8 \mathrm{~mL})$ at $0{ }^{\circ} \mathrm{C}$, concentrated sulfuric acid $(0.1 \mathrm{~mL})$ and $\mathrm{Me}_{3} \mathrm{SiN}_{3}(115 \mathrm{mg}, 1 \mathrm{mmol})$ in DCM $(2 \mathrm{~mL})$ was added during $1 \mathrm{~h}$. The reaction mixture was then stirred at the same temperature for $5 \mathrm{~h}$, After the addition of ice water, the mixture was basified with solid $\mathrm{NaHCO}_{3}$, the water layer was extracted three times with DCM. All organic layers were combined and dried over anhydrous $\mathrm{MgSO}_{4}$, filtered, and concentrated. The residue was purified by column chromatography to afford $\mathbf{6}$ as a white solid (19.4 mg, 52\% yield, $91 \%$ ee), mp: $211-213{ }^{\circ} \mathrm{C}$ (decomposed at $215{ }^{\circ} \mathrm{C}$ ). Serial number: cj-2-159, TLC $R_{f}=0.28$ $(\mathrm{PE} / \mathrm{EA}=1: 1, \mathrm{v} / \mathrm{v}) ;[\alpha]_{\mathrm{D}}{ }^{25}=-15.0\left(c 0.2, \mathrm{CH}_{3} \mathrm{OH}\right)$.

${ }^{1} \mathrm{H}$ NMR (400 MHz, DMSO-d6) $\delta 7.42-7.36$ (m, 3H, Ar-H), 7.32 (dd, $J=8.5,6.9$ $\mathrm{Hz}, 2 \mathrm{H}, \mathrm{Ar}-\mathrm{H}), 7.18-7.12\left(\mathrm{~m}, 1 \mathrm{H},=\mathrm{C}_{\underline{H C H}}\right), 2.80(\mathrm{dd}, J=17.2,7.6 \mathrm{~Hz}$, $\left.1 \mathrm{H}, \mathrm{C}^{\mathrm{HCH}} \mathrm{H}_{3}\right), 2.33\left(\mathrm{td}, J=7.4,3.2 \mathrm{~Hz}, 1 \mathrm{H},=\mathrm{CHCH}_{2}\right), 2.13(\mathrm{dd}, J=17.2$, $\left.3.3 \mathrm{~Hz}, 1 \mathrm{H},=\mathrm{CHC}_{2}\right), 1.08\left(\mathrm{~d}, J=7.3 \mathrm{~Hz}, 3 \mathrm{H}, \underline{\mathrm{CH}}_{3}\right)$

${ }^{13} \mathrm{C}$ NMR $\left(101 \mathrm{MHz}, \mathrm{DMSO}-d_{6}\right) \delta 202.0,171.4,133.4,127.9,127.9,125.1,108.1$, $37.9,35.0,17.4$.

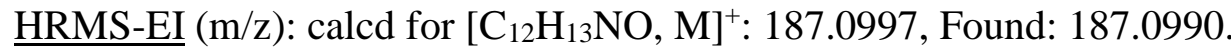

$\underline{\text { HPLC }}$ conditions: Chiralpak IC-3 column, $n$-hexane/2-propanol $=75: 25$, flow rate $=$ $0.5 \mathrm{~mL} / \mathrm{min}$, wavelength $=254 \mathrm{~nm}, t_{\mathrm{R}}=32.90 \min$ for minor isomer, $t_{\mathrm{R}}=$ $34.73 \mathrm{~min}$ for major isomer. 


\subsection{Transformation of 2aa to 7}<smiles>CC1CC=C(c2ccccc2)C1=O</smiles>

2aa

$91 \%$ ee

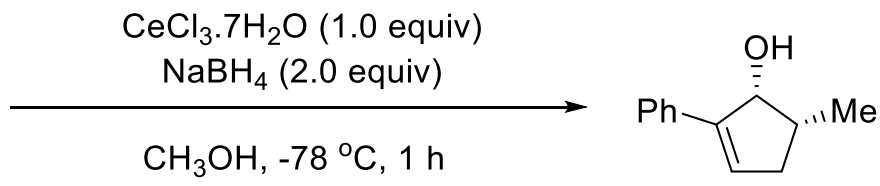

7

To a solution of $\mathbf{2 a a}(86 \mathrm{mg}, 0.5 \mathrm{mmol})$ in $\mathrm{MeOH}(5 \mathrm{~mL})$ was added $\mathrm{CeCl}_{3} \cdot 7 \mathrm{H}_{2} \mathrm{O}$ (187 mg, $0.5 \mathrm{mmol}, 1.0$ equiv). The solution was cooled to $-78^{\circ} \mathrm{C}$ for 10 min, and then $\mathrm{NaBH}_{4}(23 \mathrm{mg}, 0.6 \mathrm{mmol}, 1.2$ equiv) was added. The mixture was stirred for 20 min until the 2aa was consumed (monitored by TLC), and then was quenched with water $(5 \mathrm{~mL})$ and diluted with $\mathrm{Et}_{2} \mathrm{O}(8 \mathrm{~mL})$. The organic layer was separated and the aqueous layer was extracted with $\mathrm{Et}_{2} \mathrm{O}(2 \times 5 \mathrm{~mL})$. All organic layers were combined and dried over anhydrous $\mathrm{MgSO}_{4}$, filtered, and concentrated. The residue was purified by column chromatography to afford 7 as a white solid (83.0 mg, $95 \%$ yield, d.r. $=13: 1,91 \%$ ee $)$, mp: $86-88^{\circ} \mathrm{C}$. Serial number: cj-2-101, TLC $R_{f}=$ $0.63(\mathrm{PE} / \mathrm{EA}=5: 1, \mathrm{v} / \mathrm{v}),[\alpha]_{\mathrm{D}}^{26}=-60.7\left(c 0.3, \mathrm{CHCl}_{3}\right)$.

${ }^{1} \mathrm{H}$ NMR $\left(400 \mathrm{MHz}, \mathrm{CDCl}_{3}\right) \delta 7.61-7.54(\mathrm{~m}, 2 \mathrm{H}, \mathrm{Ar}-\mathrm{H}), 7.34(\mathrm{~m}, 2 \mathrm{H}, \mathrm{Ar}-\mathrm{H}), 7.27$ $7.22(\mathrm{~m}, 1 \mathrm{H}, \mathrm{Ar}-\mathrm{H}), 6.33\left(\mathrm{t}, J=2.7 \mathrm{~Hz}, 1 \mathrm{H},=\mathrm{CHCH}_{2}\right), 4.95-4.87(\mathrm{~m}, 1 \mathrm{H}$, C$\underline{H O H}), 2.57$ (ddd, $\left.J=17.1,7.6,3.1 \mathrm{~Hz}, 1 \mathrm{H},=\mathrm{CHCH}_{2}\right), 2.43(\mathrm{dt}, J=14.2$, $\left.7.1 \mathrm{~Hz}, 1 \mathrm{H}, \mathrm{C}_{\mathrm{HCH}}\right), 2.34-2.18\left(\mathrm{~m}, 1 \mathrm{H},=\mathrm{CHCH}_{2}\right), 1.29(\mathrm{~s}, 1 \mathrm{H}, \mathrm{O} \underline{\mathrm{H}}), 1.18$ (d, $\left.J=7.1 \mathrm{~Hz}, 3 \mathrm{H}, \mathrm{C}_{3}\right)$.

${ }^{13} \mathrm{C} \mathrm{NMR}\left(101 \mathrm{MHz}, \mathrm{CDCl}_{3}\right) \delta 145.0,135.1,130.1,128.5,127.3,126.0,78.2,38.7$, $38.1,13.8$.

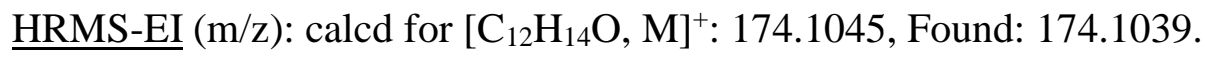

HPLC condition: Chiralpak IC-3 column, $n$-hexane/2-propanol $=99: 1$, flow rate $=1$ $\mathrm{mL} / \mathrm{min}$, wavelength $=254 \mathrm{~nm}, t_{\mathrm{R}}=7.40$ min for minor isomer, $t_{\mathrm{R}}=12.53$ min for major isomer. 


\subsection{Transformation of 7 to 8}<smiles>C[C@H]1CC=C(c2ccccc2)[C@H]1O</smiles>

7

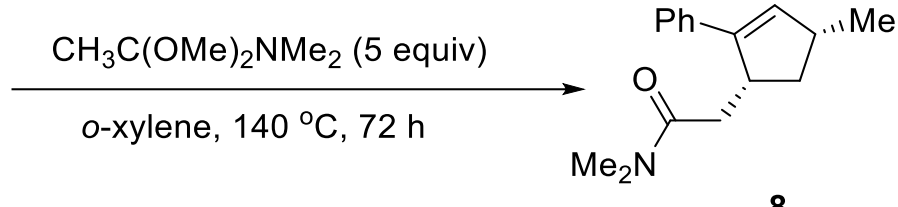

8

A solution of allyl alcohol $7(34.8 \mathrm{mg}, 0.2 \mathrm{mmol})$ and $N, N$-dimethylacetamide dimethyl acetal $(133 \mathrm{mg}, 1 \mathrm{mmol})$ in $o$-xylene $(1.4 \mathrm{~mL})$ was refluxed for $48 \mathrm{~h}$. After which, xylene was removed by vacuo and the residual oil was purified by column chromatography to afford $\mathbf{8}$ as a yellow oil (44.0 mg, 91\% yield, 93\% ee), Serial number: cj-2-160. TLC $R_{f}=0.30(\mathrm{PE} / \mathrm{EA}=7: 3, \mathrm{v} / \mathrm{v}) .[\alpha]_{\mathrm{D}}{ }^{25}=-14.0\left(c 1, \mathrm{CHCl}_{3}\right)$.

$\underline{{ }^{1} \mathrm{H} \mathrm{NMR}}\left(400 \mathrm{MHz}, \mathrm{CDCl}_{3}\right) \delta 7.37(\mathrm{~d}, J=7.0 \mathrm{~Hz}, 2 \mathrm{H}, \mathrm{Ar}-\mathrm{H}), 7.31(\mathrm{t}, J=7.7 \mathrm{~Hz}, 2 \mathrm{H}$, Ar-H), $7.22(\mathrm{t}, J=7.2 \mathrm{~Hz}, 1 \mathrm{H}, \mathrm{Ar}-\mathrm{H}), 5.94(\mathrm{t}, J=2.1 \mathrm{~Hz}, 1 \mathrm{H},=\mathrm{C} \underline{\mathrm{H}}), 3.73$ (ddd, $\left.J=5.2,3.4,1.7 \mathrm{~Hz}, 1 \mathrm{H}, \mathrm{CHCH}_{3}\right), 2.91\left(\mathrm{~s}, 3 \mathrm{H}, \mathrm{NMe}_{2}\right), 2.90-2.87(\mathrm{~m}$, 1H, $\underline{\mathrm{HCH}}_{2} \mathrm{CO}$ ), 2.86 (s, 3H, $\underline{\mathrm{NMe}}_{2}$ ), 2.73 - 2.66 (m, 2H, $\underline{\mathrm{H}}_{2} \mathrm{CO}$ ), 2.15 (dd, $\left.J=15.9,10.5 \mathrm{~Hz}, 1 \mathrm{H}, \underline{\mathrm{C}}_{2}\right), 1.21\left(\mathrm{dt}, J=13.3,5.5 \mathrm{~Hz}, 1 \mathrm{H}, \mathrm{CH}_{2}\right), 1.13(\mathrm{~d}, J$ $\left.=7.0 \mathrm{~Hz}, 3 \mathrm{H}, \mathrm{CHC}_{3}\right)$.

${ }^{13} \mathrm{C} \mathrm{NMR}\left(101 \mathrm{MHz}, \mathrm{CDCl}_{3}\right) \delta 172.5,144.9,136.4,134.1,128.4,127.0,126.4,41.9$, $39.7,39.6,39.2,37.2,35.4,22.4$.

HRMS-EI (m/z): calcd for $\left[\mathrm{C}_{16} \mathrm{H}_{21} \mathrm{NO}, \mathrm{M}\right]^{+}:$243.1623, Found: 243.1619.

$\underline{\text { HPLC }}$ condition: Chiralpak IC-3 column, $n$-hexane/2-propanol $=93: 7$, flow rate $=0.5$ $\mathrm{mL} / \mathrm{min}$, wavelength $=254 \mathrm{~nm}, t_{\mathrm{R}}=51.06 \min$ for minor isomer, $t_{\mathrm{R}}=53.21$ min for major isomer. 


\section{Procedure of React IR Experiments}
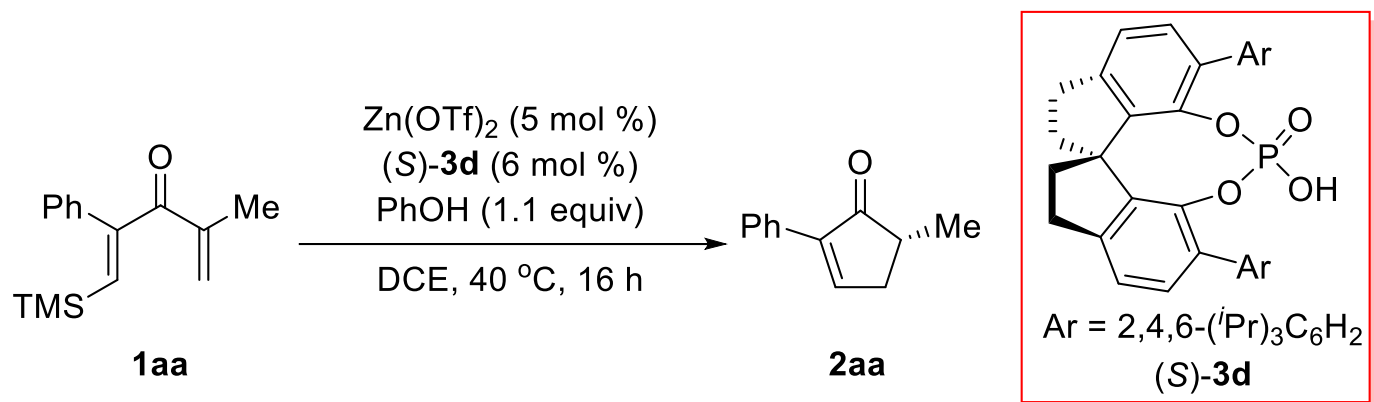

A three necked reaction vessel was fitted with a magnetic stirring bar. The IR probe was inserted through an adapter into the middle neck; the other two necks were capped by septa for reagent injections and an Ar line. To this vessel were added $\mathrm{Zn}(\mathrm{OTf})_{2}(3.6 \mathrm{mg}, 0.01 \mathrm{mmol}),(S)-3 d(8.6 \mathrm{mg}, 0.012 \mathrm{mmol})$ and $\mathrm{PhOH}(20.7 \mathrm{mg}$, $0.22 \mathrm{mmol})$. This vessel was evacuated and purged with Ar three times. To this vessel was added DCE (2 mL) via a syringe. The mixture was allowed to stir at $40{ }^{\circ} \mathrm{C}$ in a water bath. Then the IR data collection was started, followed by addition of $1 \mathrm{~mL}$ DCE solution of dienone 1aa $(48.8 \mathrm{mg}, 0.2 \mathrm{mmol})$ in one portion. In situ IR spectra were recorded over the course of the reaction. The reaction yield was confirmed by isolation or ${ }^{1} \mathrm{H}$ NMR with dibromomethane as an internal standard.

ConcIRT Spectrum of the 2aa with peak at 1704 wave number $\mathrm{cm}^{-1}$ was used for the generation of the kinetic profiles.

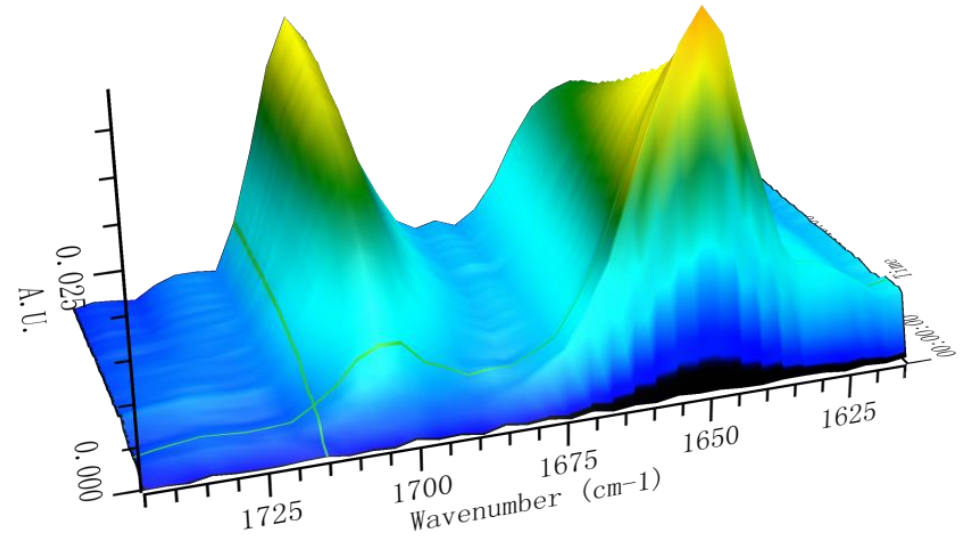

METTLER TOLEDO

\section{Dependence of the Reaction Rate on Concentration of 1aa}



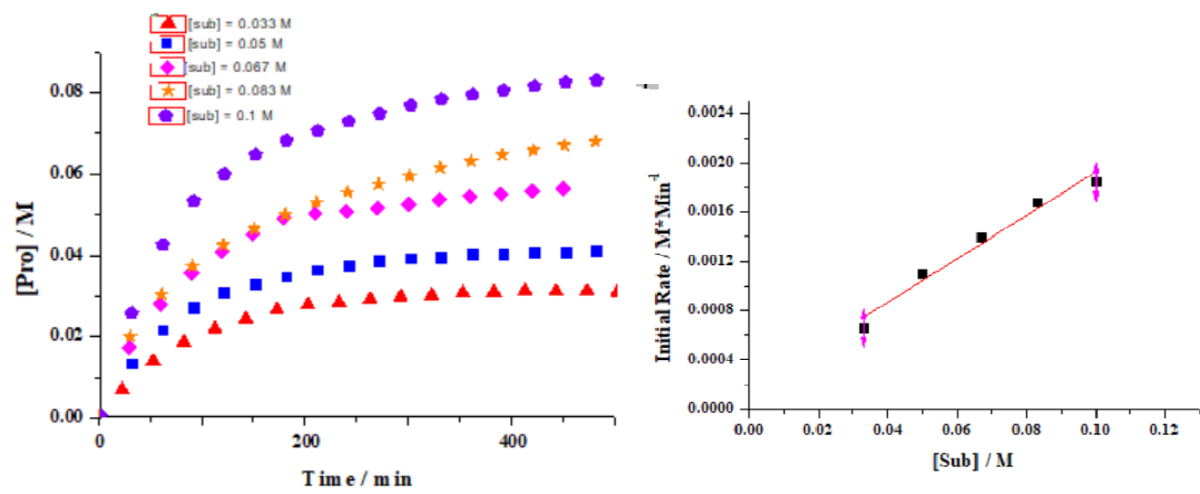

\begin{tabular}{cc}
\hline$[1 \mathrm{a}] / \mathrm{M}$ & Initial rate $/ \mathrm{M}^{*} \mathrm{~min}^{-1}$ \\
\hline 0.033 & $6.62 * 10-4$ \\
0.05 & $1.10 * 10-3$ \\
0.067 & $1.40 * 10-3$ \\
0.083 & $1.68 * 10-3$ \\
0.1 & $1.84 * 10-3$ \\
\hline
\end{tabular}

Kinetic profiles of different initial concentrations of 1aa (from $0.033 \mathrm{M}$ to $0.1 \mathrm{M}$ ).

Reaction condition: $\mathrm{Zn}(\mathrm{OTf})_{2}(0.01 \mathrm{mmol}),(S)-3 \mathrm{~d}(0.012 \mathrm{mmol}), \mathrm{PhOH}(0.6 \mathrm{mmol})$, carried out in DCE $(3 \mathrm{~mL})$ at $40{ }^{\circ} \mathrm{C}$.

\section{Dependence of the Reaction Rate on Concentration of $\mathrm{Zn}(\mathrm{OTf})_{2}$}
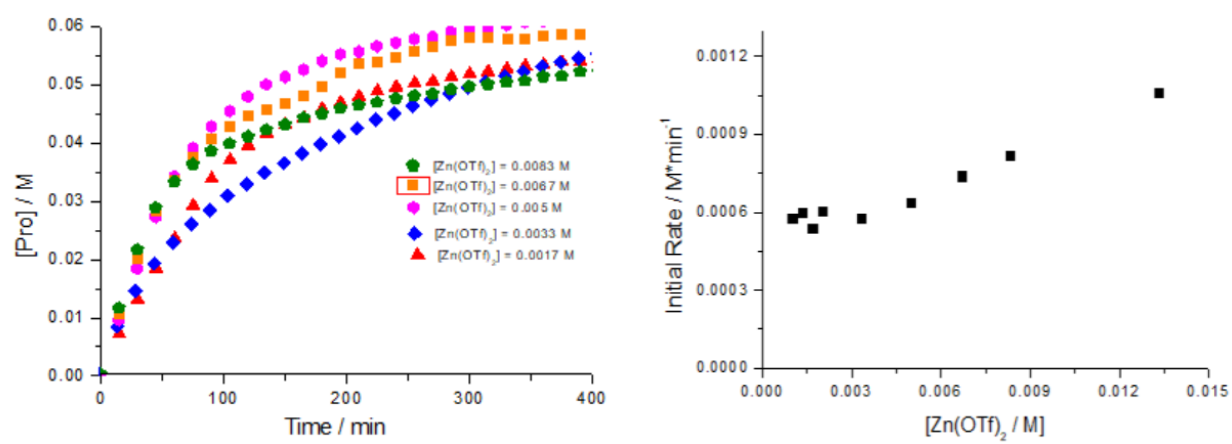


\begin{tabular}{cc}
\hline$\left[\mathrm{Zn}(\mathrm{OTf})_{2}\right] / \mathrm{M}$ & Initial rate $/ \mathrm{M}^{*} \mathrm{~min}^{-1}$ \\
\hline 0.0010 & $5.8 * 10-4$ \\
0.0013 & $6.0 * 10-4$ \\
0.0017 & $5.4 * 10-4$ \\
0.0020 & $6.1 * 10-4$ \\
0.0027 & $5.9 * 10-4$ \\
\hline
\end{tabular}

\begin{tabular}{cc}
\hline$\left[\mathrm{Zn}(\mathrm{OTf})_{2}\right] / \mathrm{M}$ & Initial rate $/ \mathrm{M}^{*} \mathrm{~min}^{-1}$ \\
\hline 0.0033 & $5.8 * 10-4$ \\
0.005 & $6.4 * 10-4$ \\
0.0067 & $7.4 * 10-4$ \\
0.0083 & $8.2 * 10-4$ \\
0.0133 & 0.00106 \\
\hline
\end{tabular}

Kinetic profiles of different initial concentrations of $\mathrm{Zn}(\mathrm{OTf})_{2}$ (from $0.001 \mathrm{M}$ to $0.0133 \mathrm{M})$.

Reaction condition: 1aa $(0.2 \mathrm{mmol}),(S)-3 \mathbf{d}(0.012 \mathrm{mmol}), \mathrm{PhOH}(0.22 \mathrm{mmol})$, carried out in DCE $(3 \mathrm{~mL})$ at $40{ }^{\circ} \mathrm{C}$.

\section{Dependence of the Reaction Rate on Concentration of $(S)-3 d$}
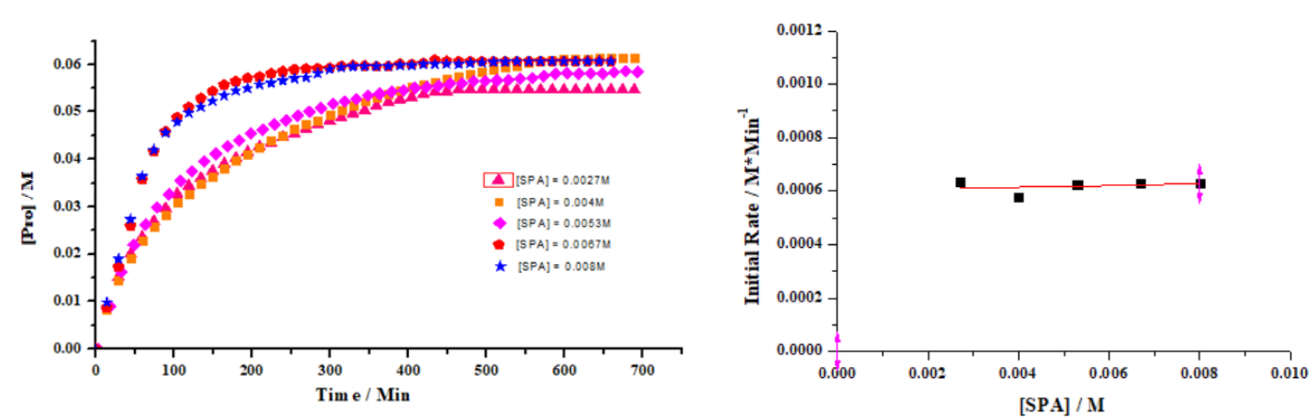

\begin{tabular}{cc}
\hline$[S P A] / M$ & Initial rate/M*min ${ }^{-1}$ \\
\hline 0.0027 & $6.33 * 10-4$ \\
0.004 & $5.8 * 10-4$ \\
0.0053 & $6.25 * 10-4$ \\
0.0067 & $6.3 * 10-4$ \\
0.008 & $6.28 * 10-4$ \\
\hline
\end{tabular}

Kinetic profiles of different initial concentrations of (S)-3d (from $0.0027 \mathrm{M}$ to 0.008 M).

Reaction condition: 1aa (0.2 mmol), $\mathrm{Zn}(\mathrm{OTf})_{2}(0.01 \mathrm{mmol}), \mathrm{PhOH}(0.22 \mathrm{mmol})$, carried out in DCE $(3 \mathrm{~mL})$ at $40{ }^{\circ} \mathrm{C}$. 


\section{Dependence of the Reaction Rate on Concentration of PhOH}
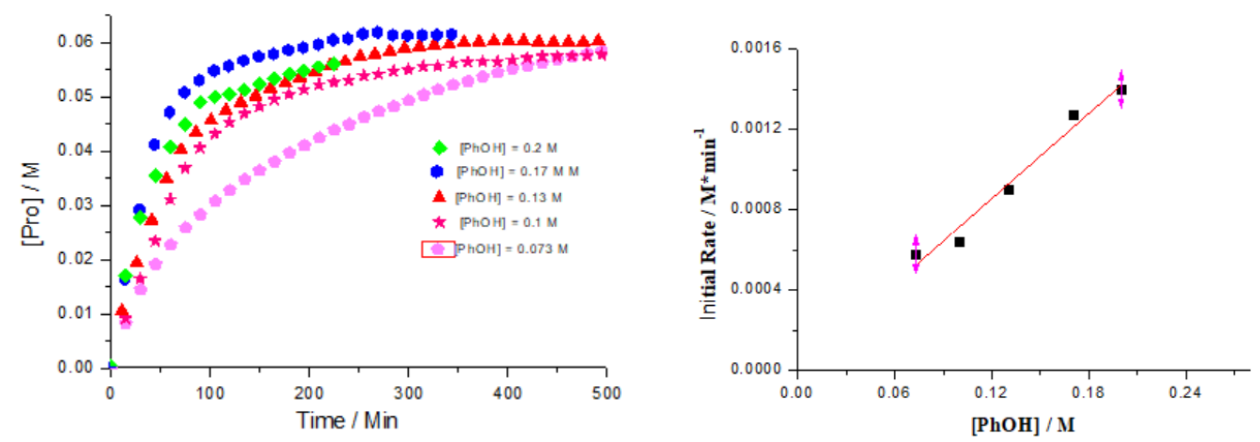

\begin{tabular}{cc}
\hline$[S P A] / M$ & Initial rate $/ \mathrm{M}^{*} \mathrm{~min}^{-1}$ \\
\hline 0.073 & $5.8 * 10-4$ \\
0.1 & $6.42 * 10-4$ \\
0.13 & $9.0 * 10-4$ \\
0.17 & $1.27 * 10-3$ \\
0.2 & $1.4 * 10-3$ \\
\hline
\end{tabular}

Kinetic profiles of different initial concentrations of $\mathrm{PhOH}$ (from 0.073 $\mathrm{M}$ to 0.2 M).

Reaction condition: 1aa $(0.2 \mathrm{mmol}), \mathrm{Zn}(\mathrm{OTf})_{2}(0.01 \mathrm{mmol}),(S)-3 \mathbf{d}(0.012 \mathrm{mmol})$, carried out in DCE $(3 \mathrm{~mL})$ at $40{ }^{\circ} \mathrm{C}$. 


\section{NMR Spectra of New Compounds}

\section{(E)-4-methyl-2-phenyl-1-(trimethylsilyl)penta-1,4-dien-3-one (1aa)}

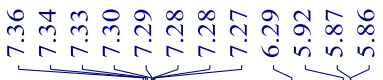<smiles>C=C(C)C(=O)/C(=C/CS(C)(=O)=O)c1ccccc1</smiles>
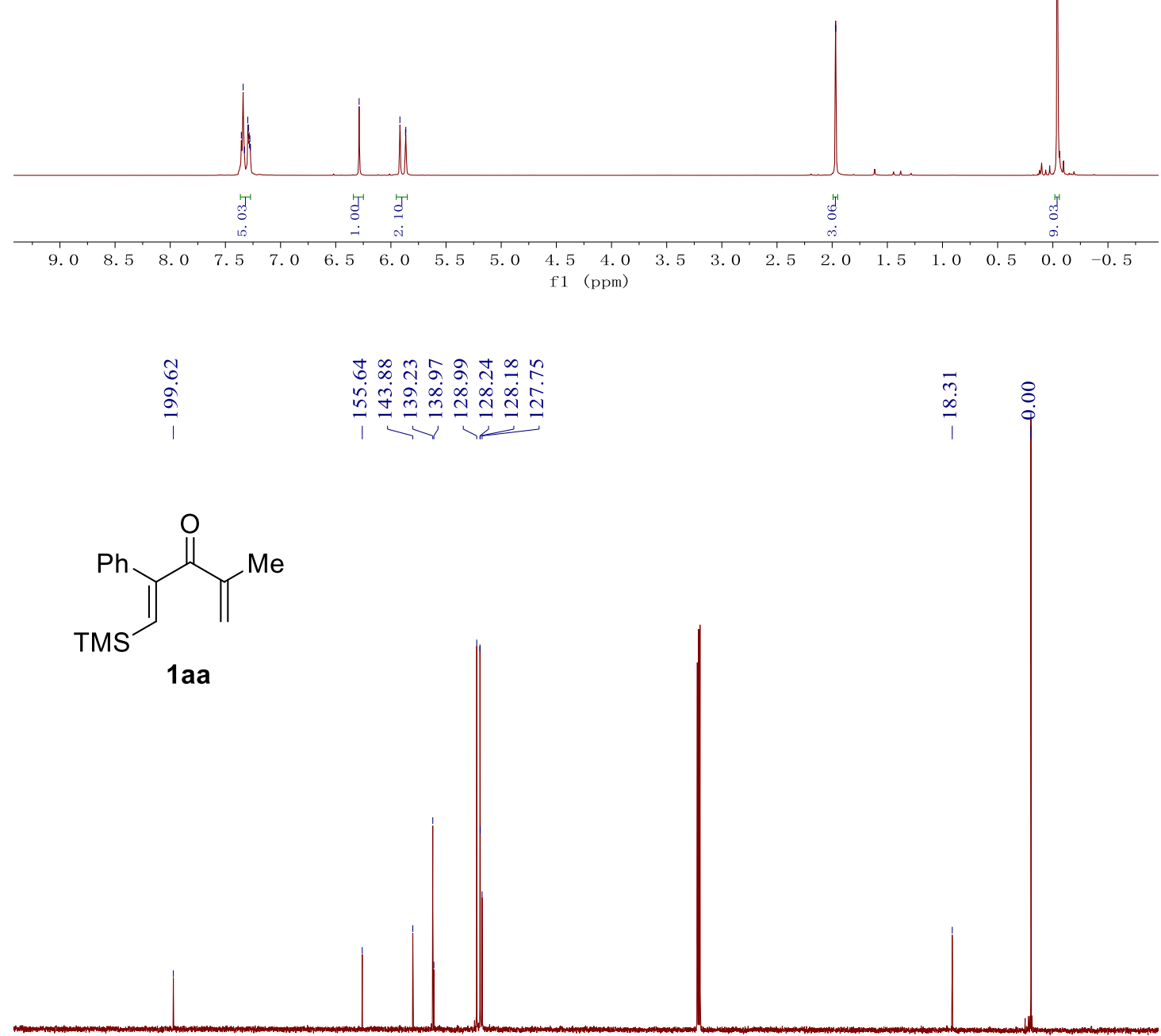

$\begin{array}{lllllllllllllllllllllllllllll}230 & 220 & 210 & 200 & 190 & 180 & 170 & 160 & 150 & 140 & 130 & 120 & 110 & 100 & 90 & 80 & 70 & 60 & 50 & 40 & 30 & 20 & 10 & 0 & -10 & -20\end{array}$ 
(E)-2-(4-methoxyphenyl)-4-methyl-1-(trimethylsilyl)penta-1,4-dien-3-one (1ab)

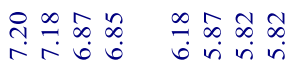

$\vec{\infty}$

$\stackrel{+}{\stackrel{5}{2}}$<smiles>C=C(C)C(=O)/C(=C\CS(C)(=O)=O)c1ccc(OC)cc1</smiles>

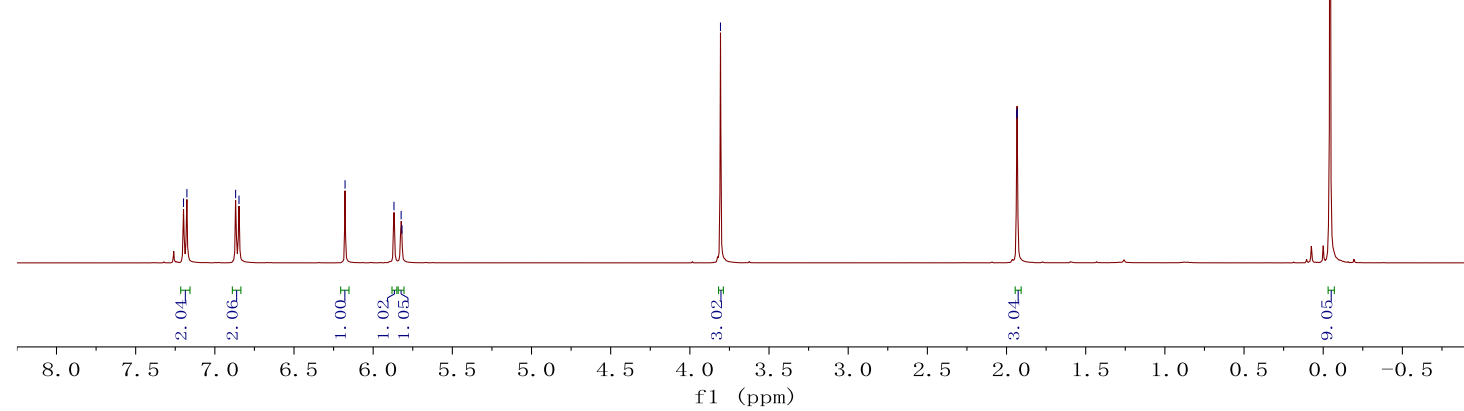
$\underset{\stackrel{\alpha}{े}}{\stackrel{\alpha}{े}}$

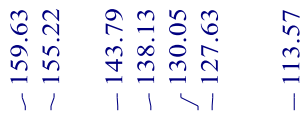
if
$\begin{array}{ll}\stackrel{1}{0} & 8 \\ 1 & 0 \\ 1 & 1\end{array}$

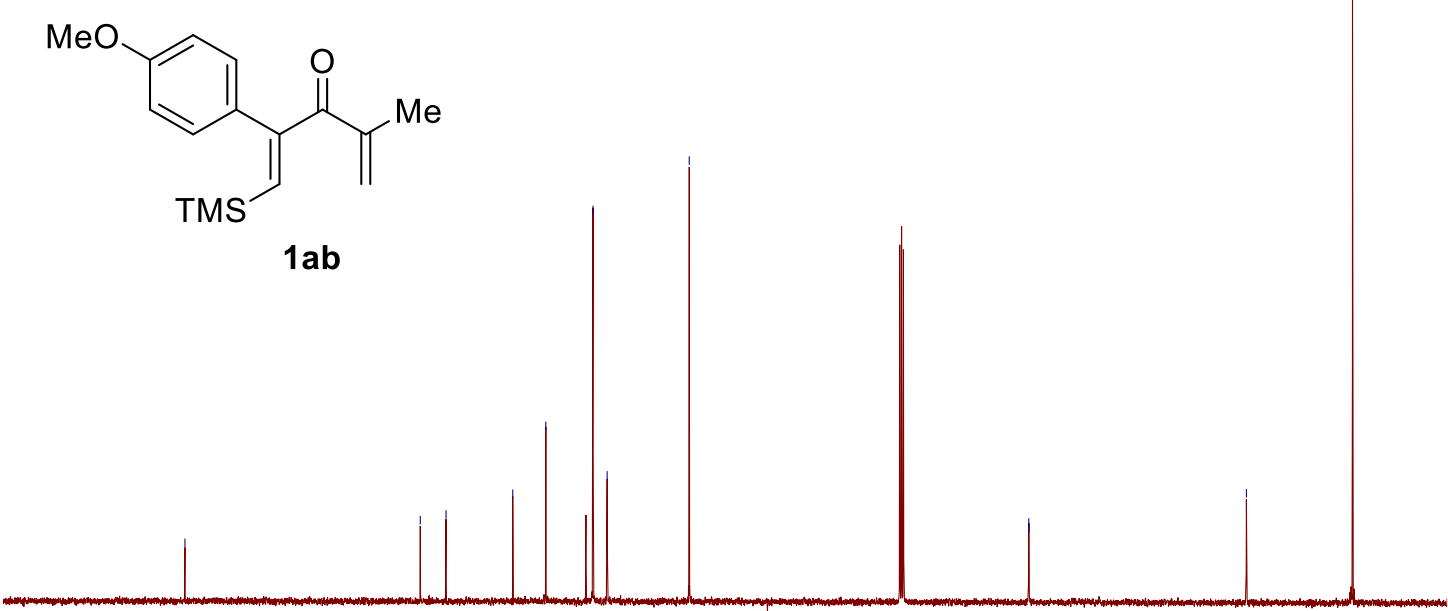

$\begin{array}{llllllllllllllllllllllllllll}3 & 220 & 210 & 200 & 190 & 180 & 170 & 160 & 150 & 140 & 130 & 120 & 110 & 100 & 90 & 80 & 70 & 60 & 50 & 40 & 30 & 20 & 10 & 0 & -10\end{array}$ f1 (ppm) 
(E)-4-methyl-2-(p-tolyl)-1-(trimethylsilyl)penta-1,4-dien-3-one (1ac)

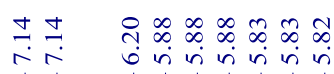

ñ<smiles>C=C(C)C(=O)C(=C[As])C(=C)c1ccc(C)cc1</smiles>
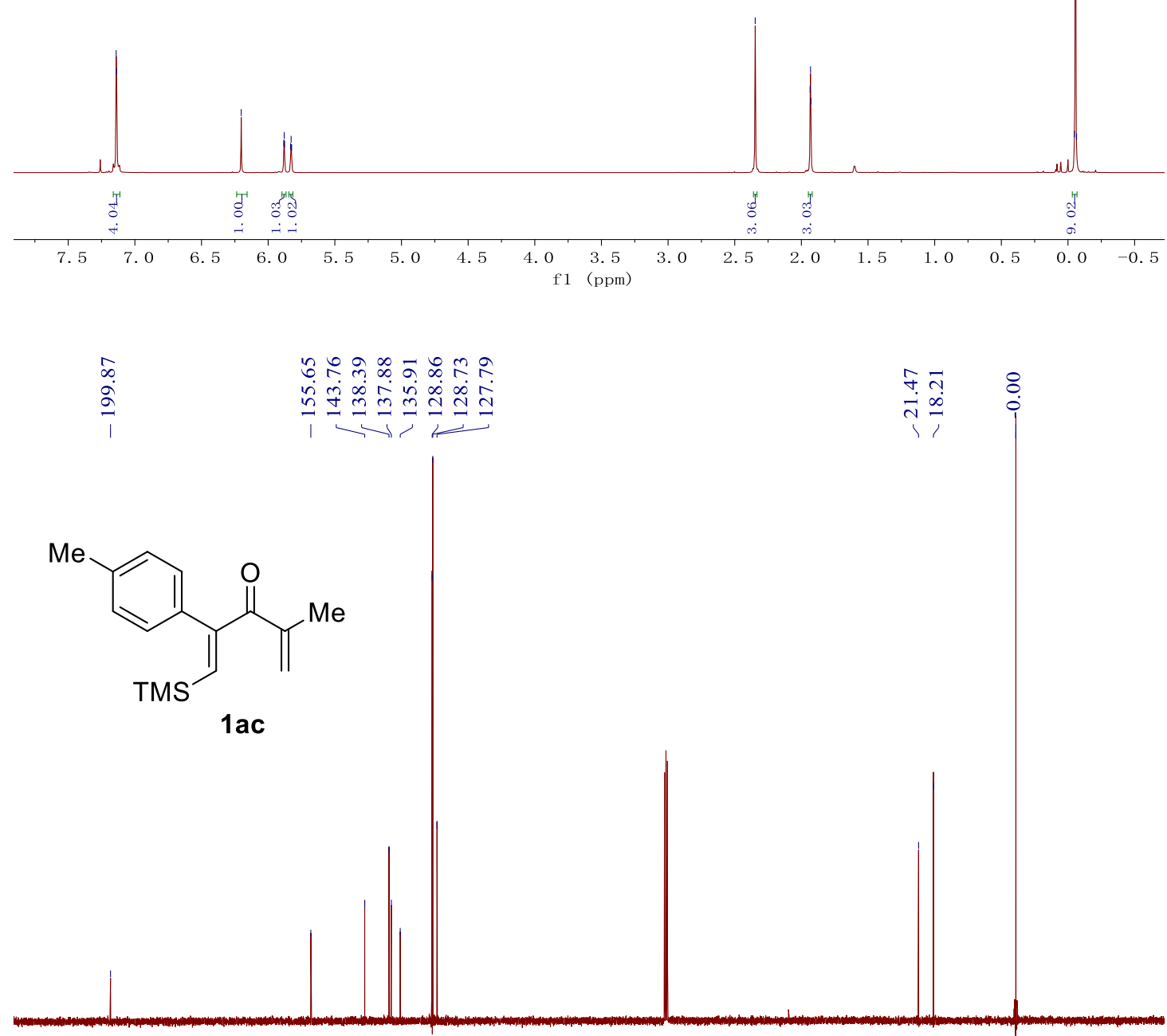

$\begin{array}{lllllllllllllllllllllllllll}120 & 210 & 200 & 190 & 180 & 170 & 160 & 150 & 140 & 130 & 120 & 110 & 100 & 90 & 80 & 70 & 60 & 50 & 40 & 30 & 20 & 10 & 0 & -10 & -20 & -31\end{array}$ f1 (ppm) 
(E)-2-(4-fluorophenyl)-4-methyl-1-(trimethylsilyl)penta-1,4-dien-3-one (1ad)

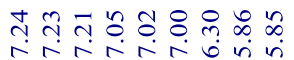

$r \operatorname{rin}$

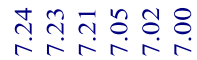

nNar

$\begin{array}{lll}0 & \infty & n \\ m & \infty & \infty \\ 0 & i & n \\ 1 & & n\end{array}$

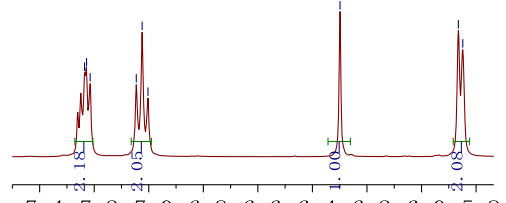<smiles>C=C(C)C(=O)C(=C[13CH3])c1ccc(F)cc1</smiles>

$\begin{array}{llllllllllll}7.4 & 7.2 & 7.0 & 6.8 & 6.6 & 6.4 & 6.2 & 6.0 & 5.8\end{array}$

f1 (ppm)

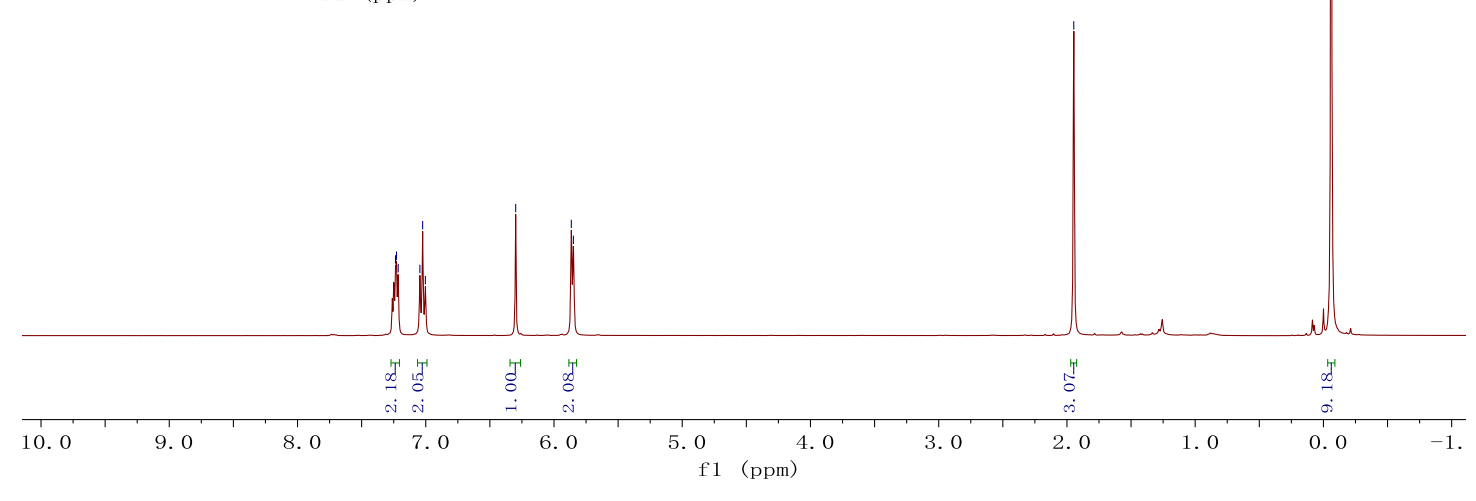

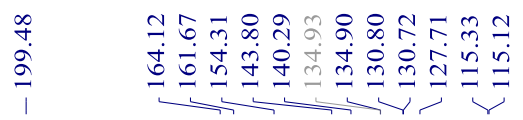

$\stackrel{\infty}{\infty} \underset{\infty}{\infty}$

$\mathrm{F}$<smiles>C=C(C)C(=O)C(=C[13CH3])c1ccc(C)cc1</smiles>

$\begin{array}{llllllllllllllllllllllllll}30 & 220 & 210 & 200 & 190 & 180 & 170 & 160 & 150 & 140 & 130 & 120 & 110 & 100 & 90 & 80 & 70 & 60 & 50 & 40 & 30 & 20 & 10 & 0 & -10 & -20\end{array}$ 1 (ppm) 
(E)-2-(4-chlorophenyl)-4-methyl-1-(trimethylsilyl)penta-1,4-dien-3-one (1ae)
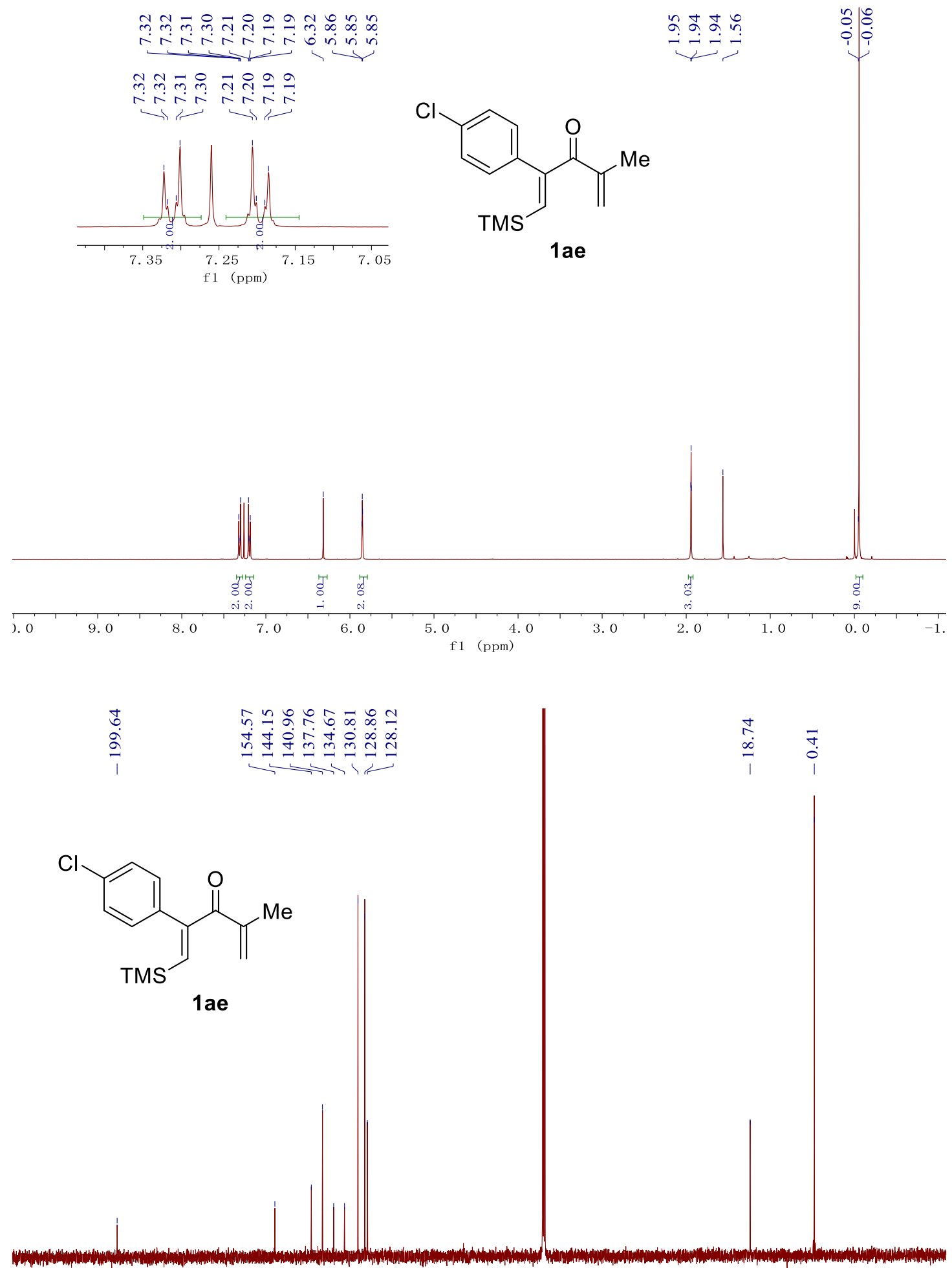

$220 \begin{array}{lllllllllllllllllllllllll}1 & 200 & 190 & 180 & 170 & 160 & 150 & 140 & 130 & 120 & 110 & 100 & 90 & 80 & 70 & 60 & 50 & 40 & 30 & 20 & 10 & 0 & -10 & -20 & -30\end{array}$ $\mathrm{f} 1$ (ppm) 
(E)-2-(4-bromophenyl)-4-methyl-1-(trimethylsilyl)penta-1,4-dien-3-one (1af)
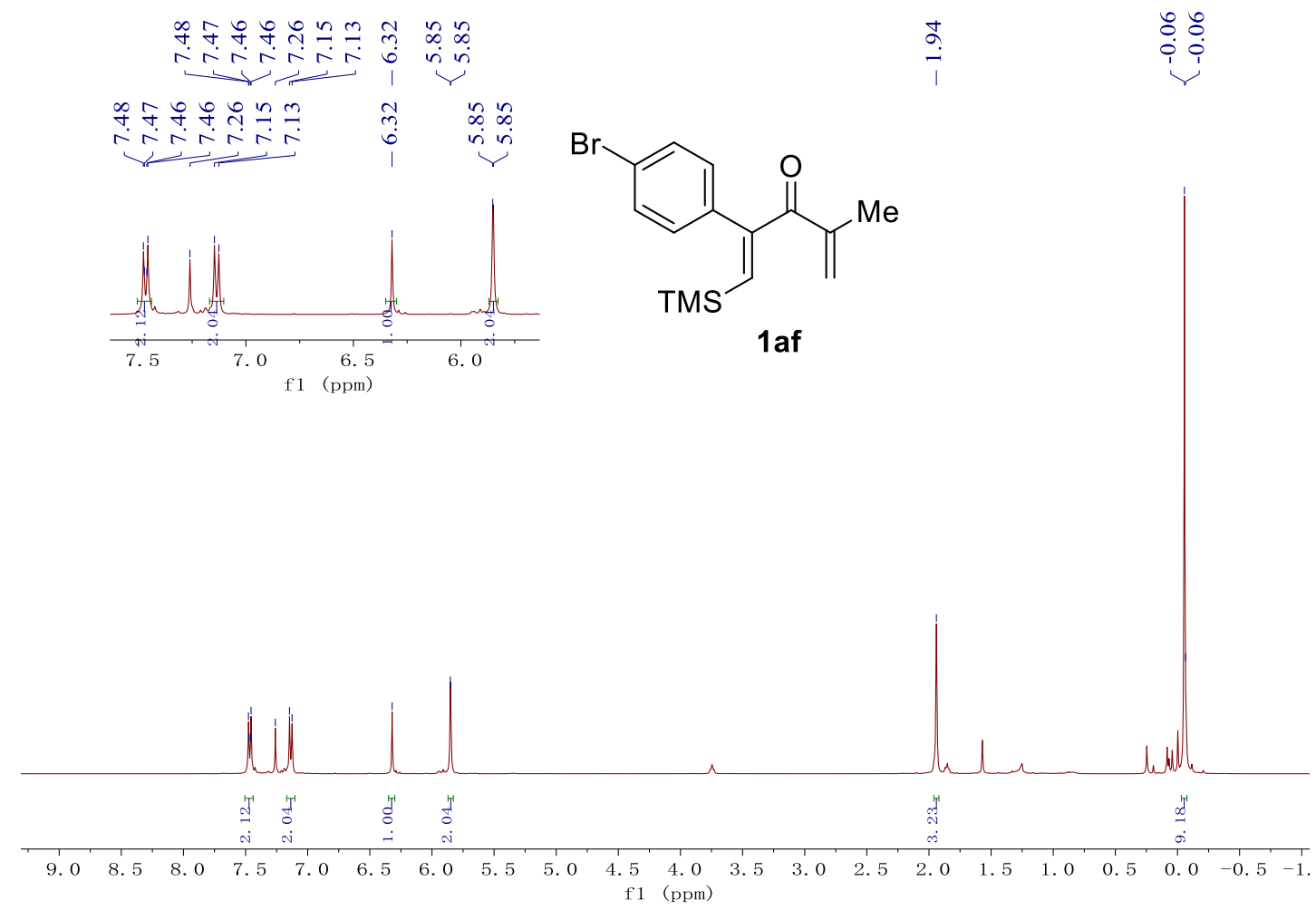

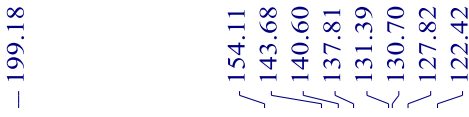

$\begin{array}{ll}m & 0 \\ m & 0 \\ i & 1 \\ 1 & 1\end{array}$<smiles>C=C(C)C(=O)/C(=C/C)c1ccc(Br)cc1</smiles>

$\begin{array}{llllllllllllllllllllllllllllllll}30 & 220 & 210 & 200 & 190 & 180 & 170 & 160 & 150 & 140 & 130 & 120 & 110 & 100 & 90 & 80 & 70 & 60 & 50 & 40 & 30 & 20 & 10 & 0 & -10 & -20 & -30\end{array}$ $\mathrm{f} 1$ (ppm) 
(E)-4-methyl-2-(4-(trifluoromethyl)phenyl)-1-(trimethylsilyl)penta-1,4-dien-3-on e (1ag)

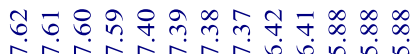

Nara

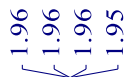

$\mathrm{F}_{3} \mathrm{C}$<smiles>C=C(C)C(=O)C(=C[AsH3])c1ccc(C)cc1</smiles>
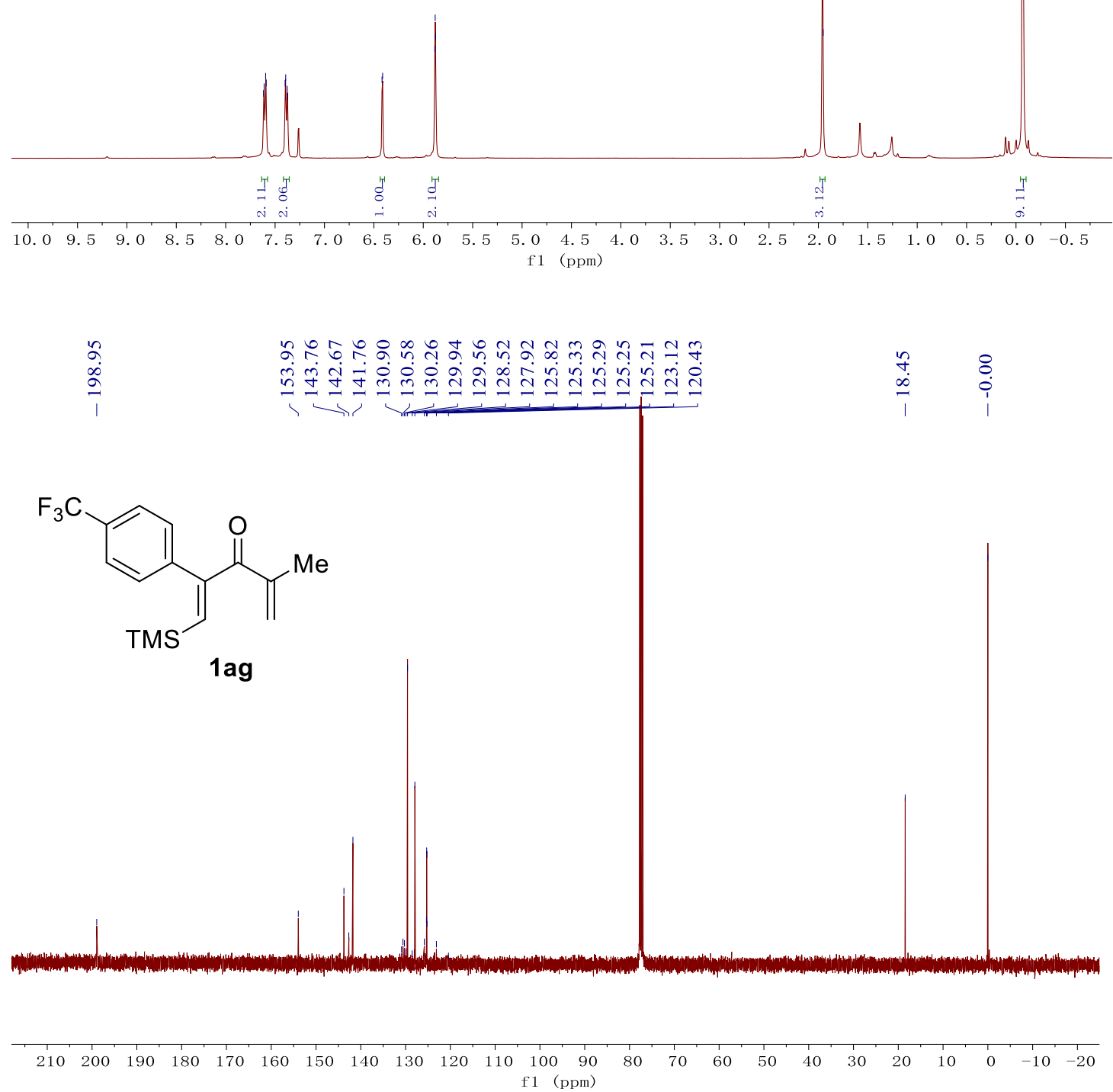


\section{(E)-4-methyl-2-(m-tolyl)-1-(trimethylsilyl)penta-1,4-dien-3-one (1ah)}

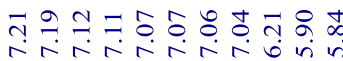

ì<smiles>C=C(C)C(=O)C(=C[As])c1cccc(C)c1</smiles>
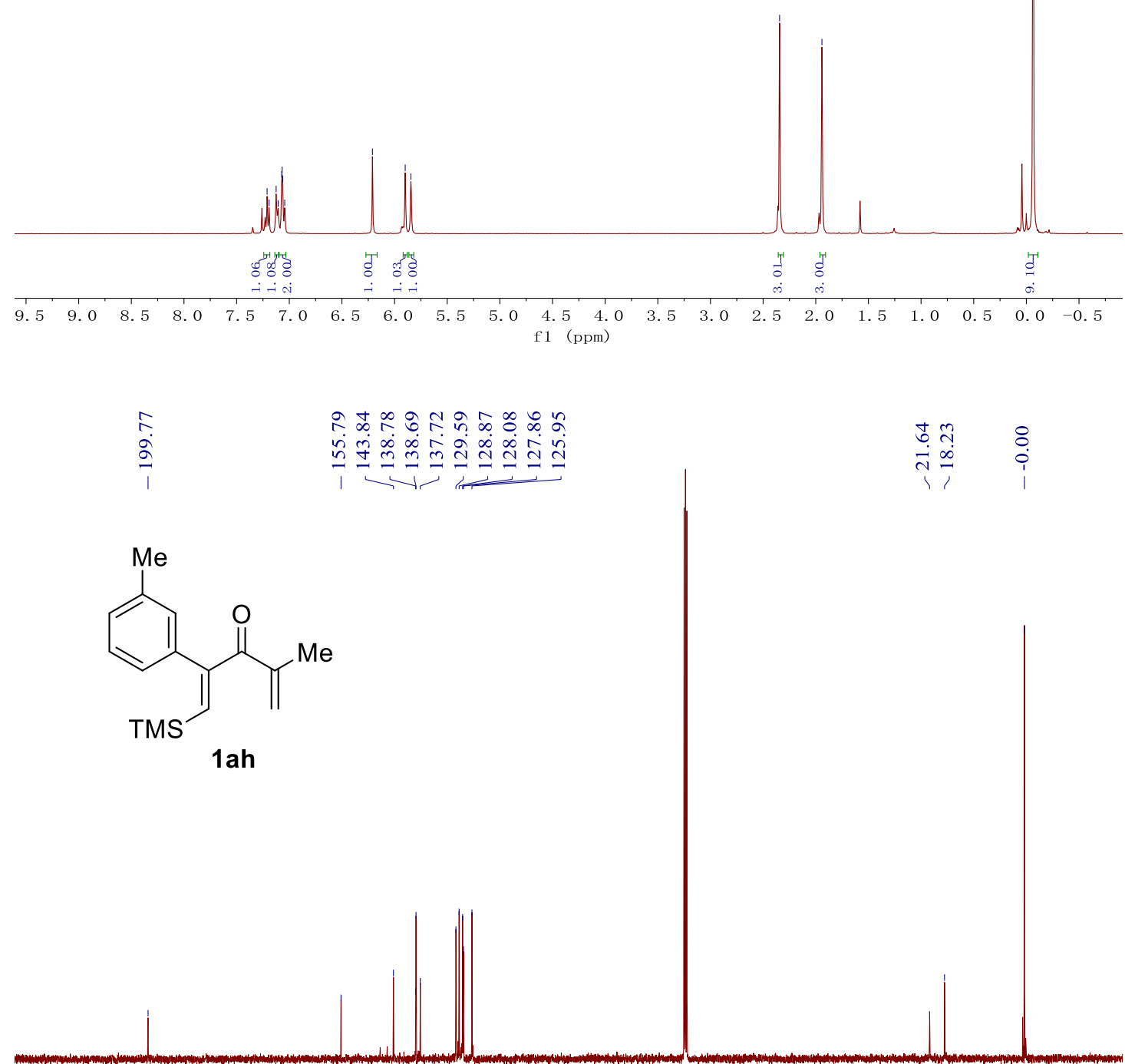

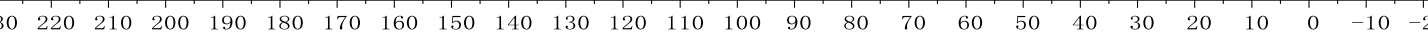




\section{(E)-4-methyl-2-(0-tolyl)-1-(trimethylsilyl)penta-1,4-dien-3-one (1ai)}

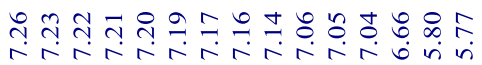

$\stackrel{\infty}{\sim} \hat{\alpha}$<smiles>C=C(C)C(=O)/C(=C/CS(C)(=O)=O)c1ccccc1C</smiles>

1 ai
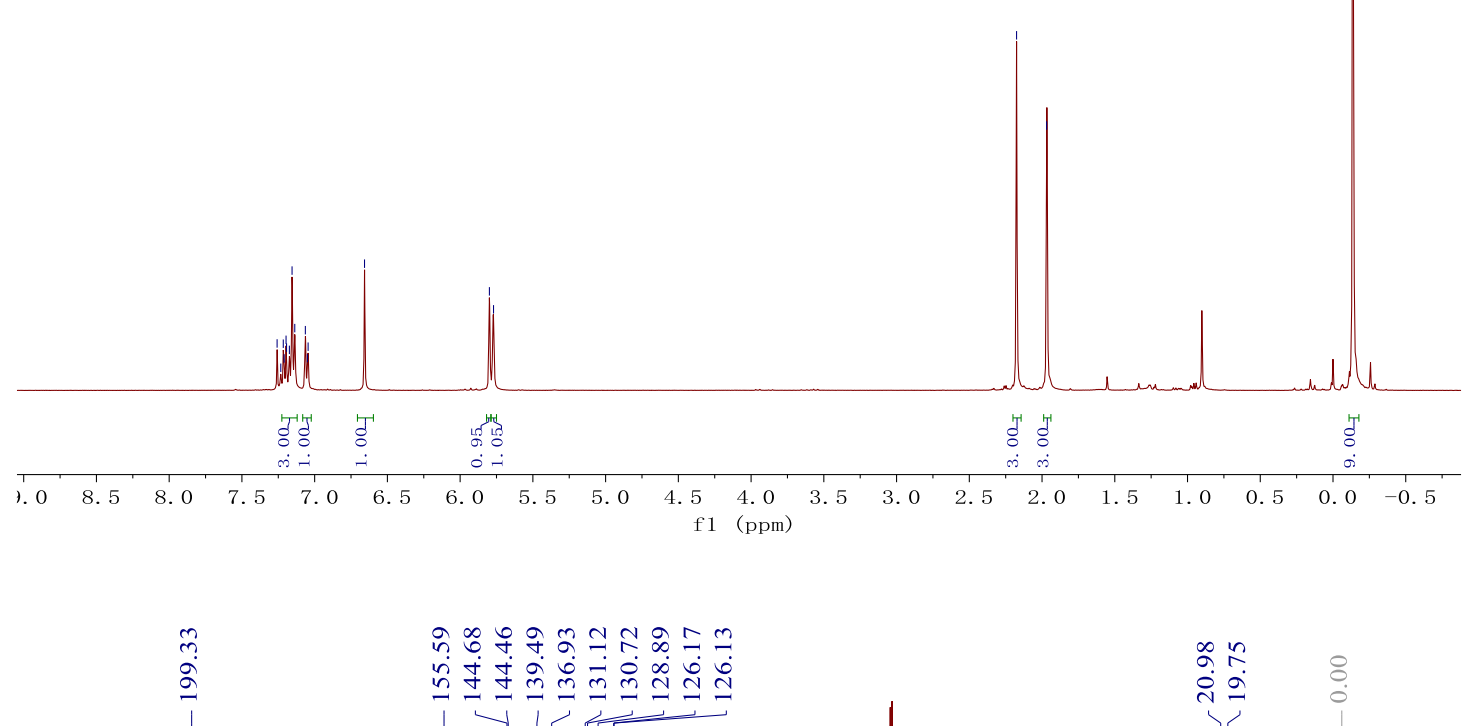<smiles>C=C(C)C(=O)C(=C[As])c1ccccc1[N+](C)(C)C</smiles>

$1 \mathrm{ai}$

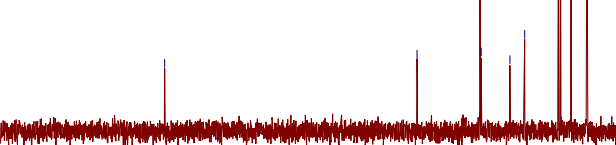




\section{(E)-2-(benzo[d][1,3]dioxol-5-yl)-4-methyl-1-(trimethylsilyl)penta-1,4-dien-3-one}

(1aj)

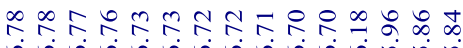

060600600000 in

$\stackrel{+}{\stackrel{2}{2}}$<smiles>C=C(C)C(=O)/C(=C/S)c1ccc2c(c1)OCO2</smiles>

1aj

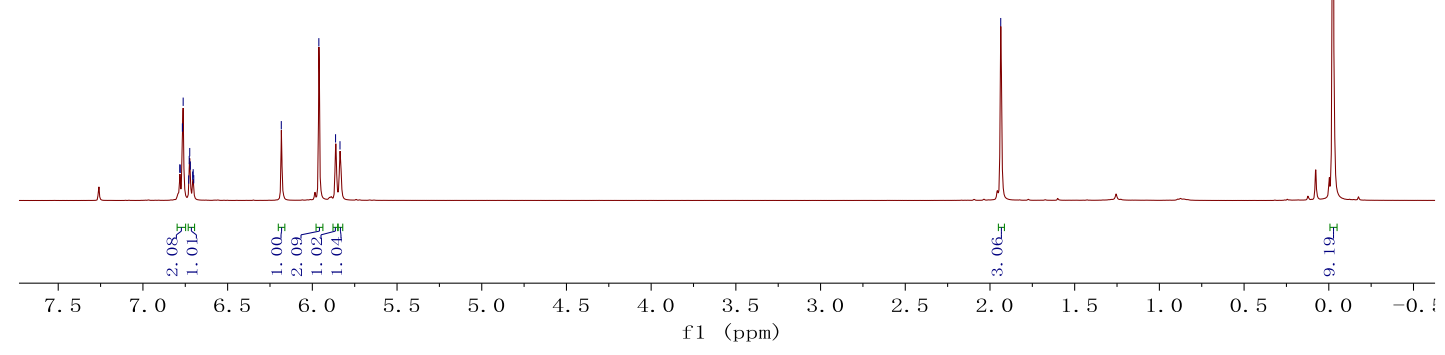

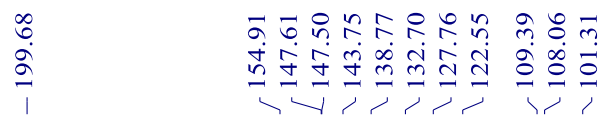<smiles>C=C(C)C(=O)/C(=C/S)c1ccc2c(c1)OCO2</smiles>

1aj

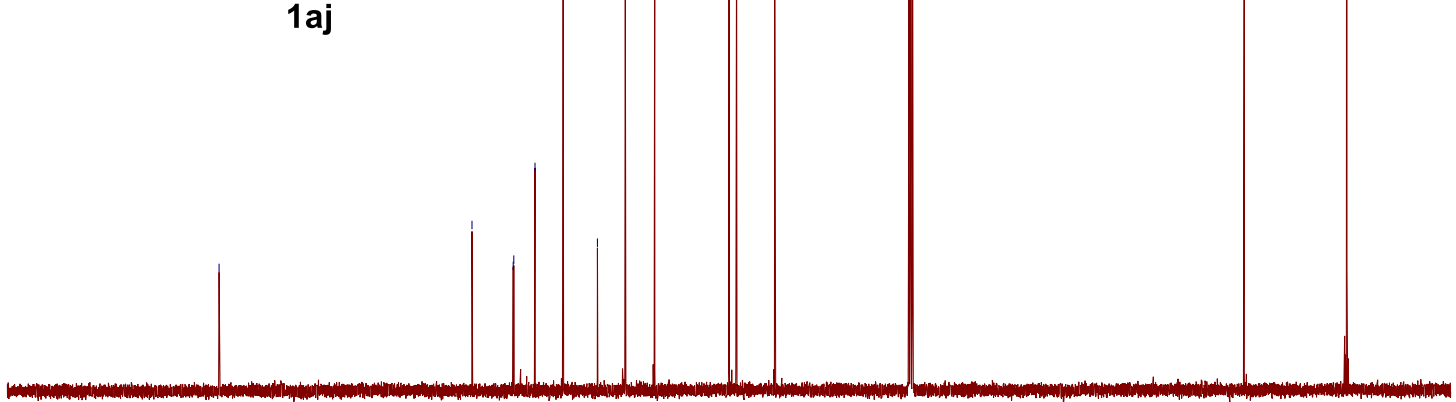

$\begin{array}{llllllllllllllllllllllllll}1 & 230 & 220 & 210 & 200 & 190 & 180 & 170 & 160 & 150 & 140 & 130 & 120 & 110 & 100 & 90 & 80 & 70 & 60 & 50 & 40 & 30 & 20 & 10 & 0 & -10\end{array}$ f1 (ppm) 
(E)-4-methyl-2-(naphthalen-2-yl)-1-(trimethylsilyl)penta-1,4-dien-3-one (1ak)

mº

$\hat{\operatorname{ăg}}$

0
0
0
1<smiles>C=C(C)C(=O)C(=C[As])c1ccc2ccccc2c1</smiles>

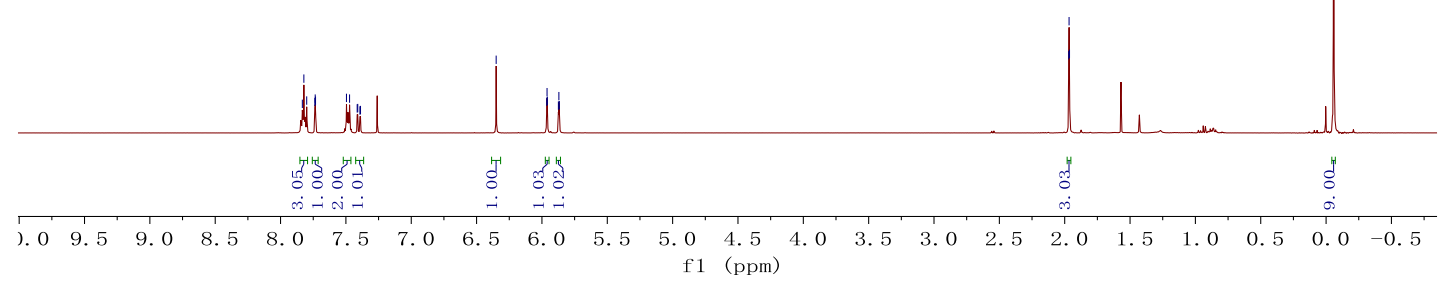

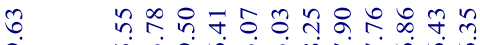

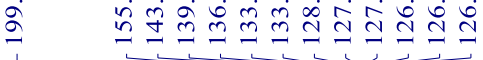

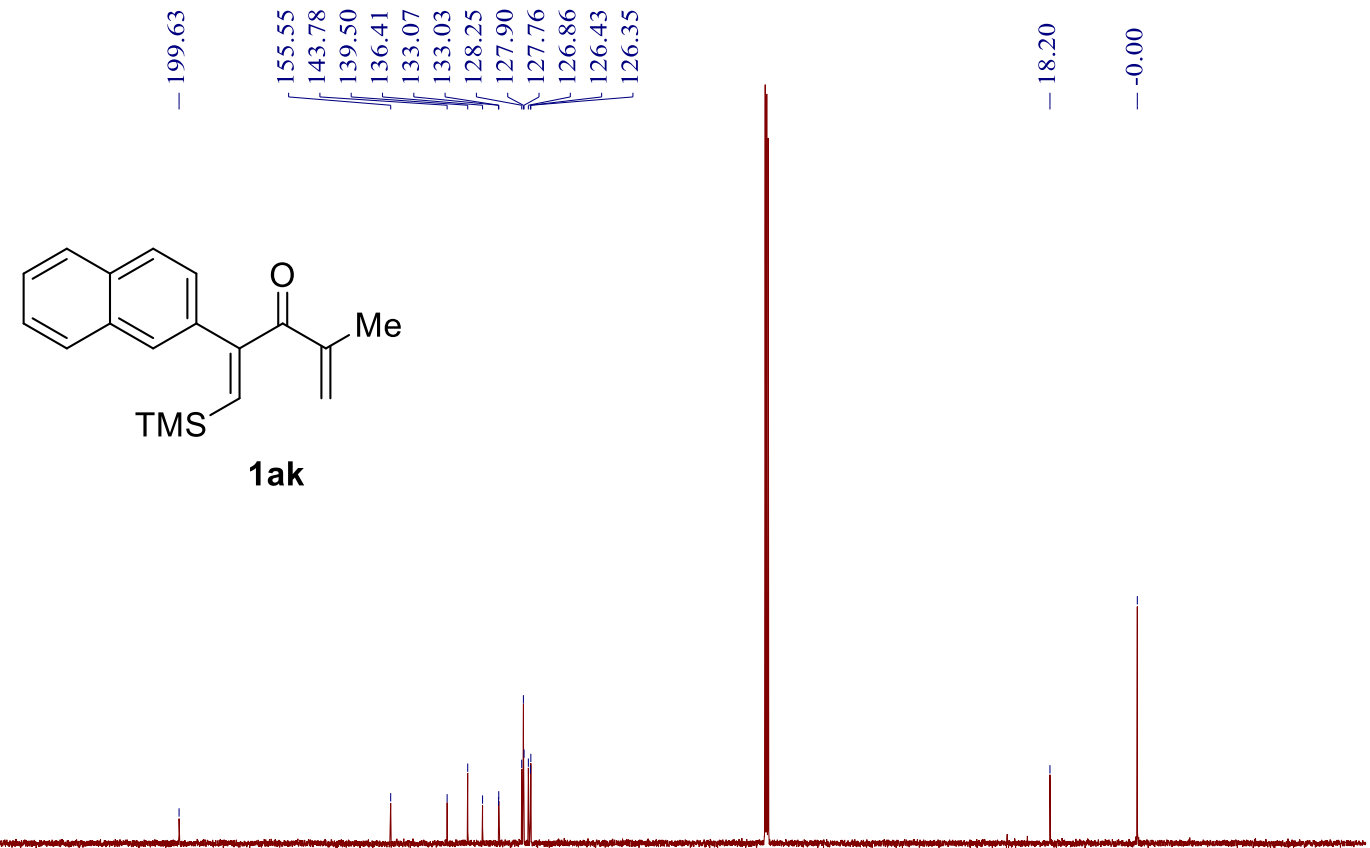

$\begin{array}{lllllll}240 & 220 & 200 & 180 & 160 & 140 & 120\end{array}$

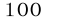

$80 \quad 60$

40

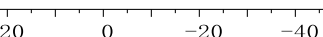

f1 (ppm) 
(Z)-4-methyl-2-(thiophen-2-yl)-1-(trimethylsilyl)penta-1,4-dien-3-one (1al)

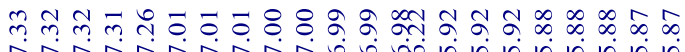

ㅇํำㅇำ

$\left\langle\left.\right|_{1 \mathrm{TMS}} ^{\mathrm{O}} \mathrm{Me}\right.$

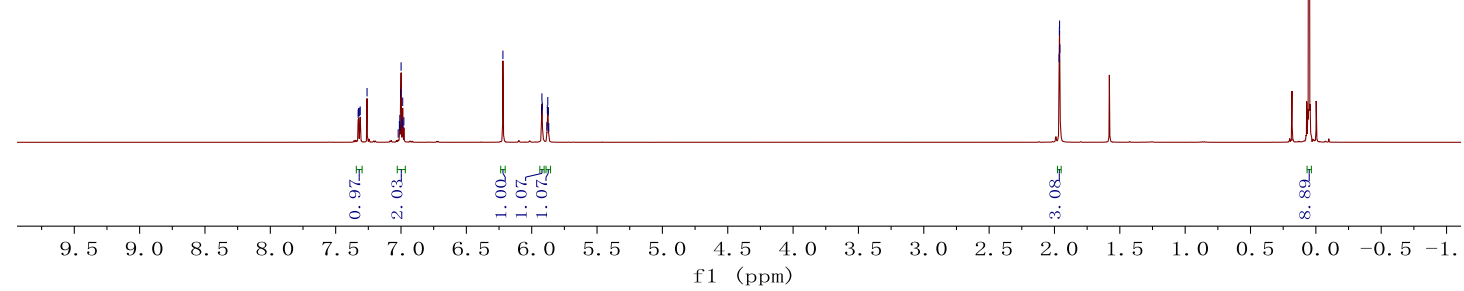

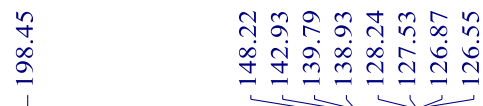

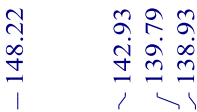

in in

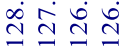

in
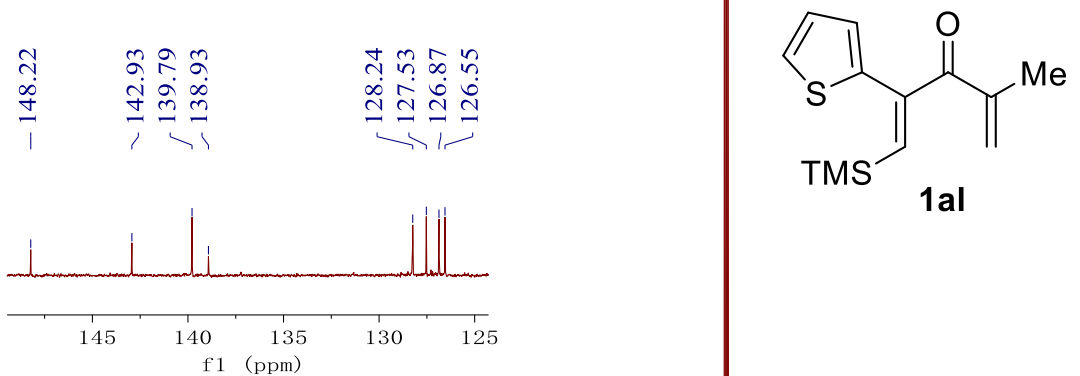

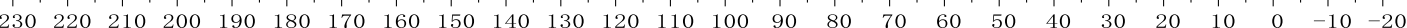




\section{(E)-4-methylene-2-phenyl-1-(trimethylsilyl)hex-1-en-3-one (1ba)}

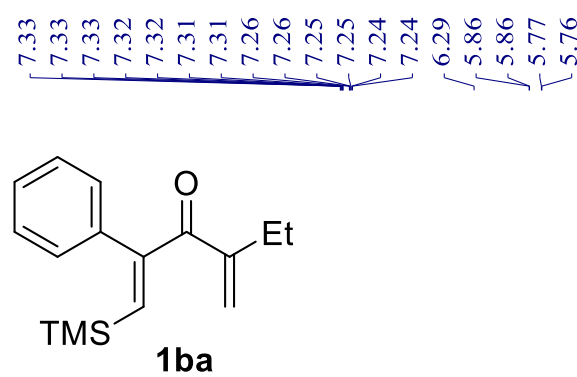

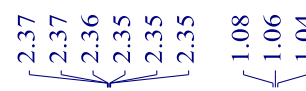
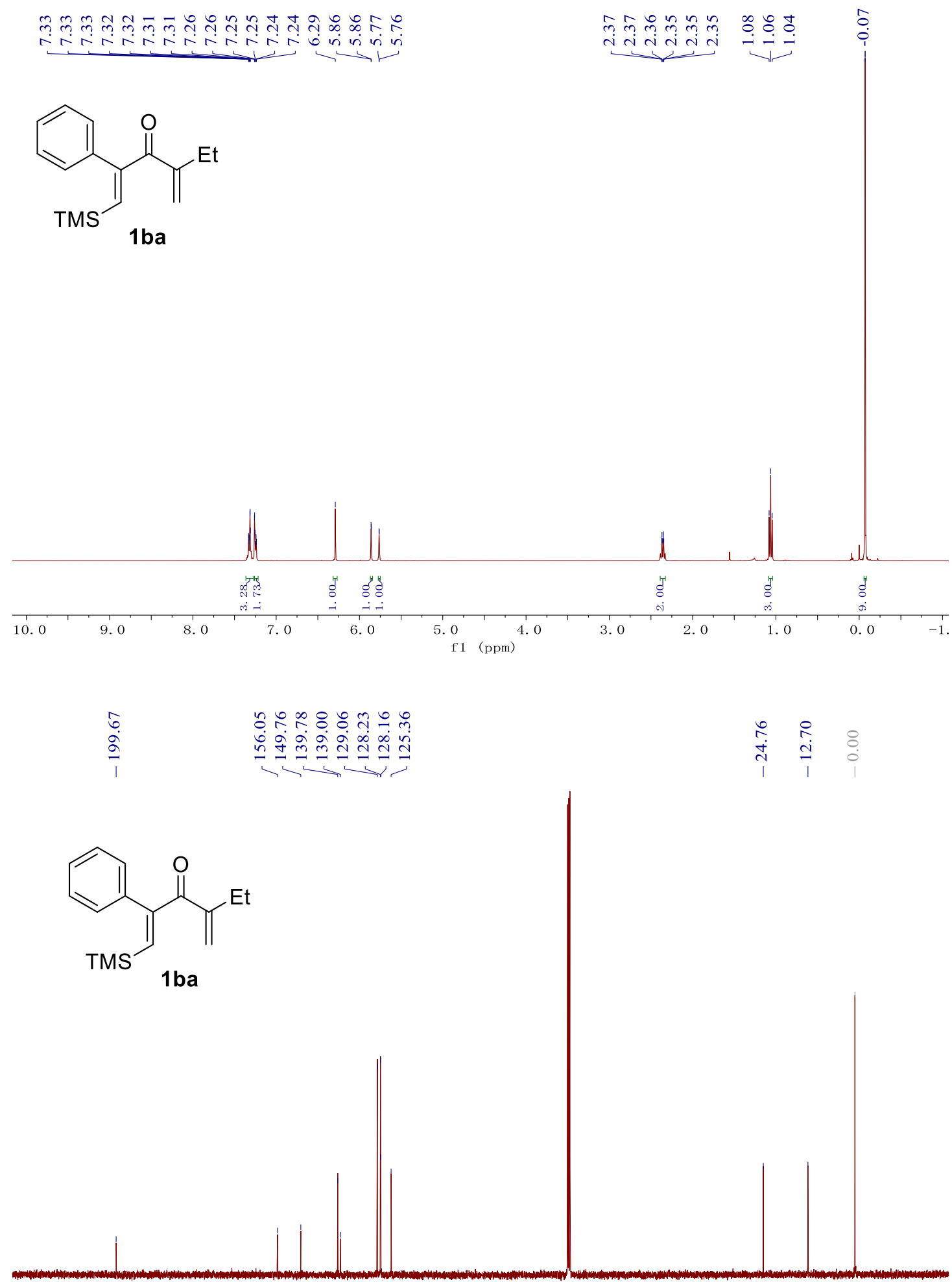

$\begin{array}{llllllllllllllllllllllllll}1 & 220 & 210 & 200 & 190 & 180 & 170 & 160 & 150 & 140 & 130 & 120 & 110 & 100 & 90 & 80 & 70 & 60 & 50 & 40 & 30 & 20 & 10 & 0 & -10 & -20\end{array}$ f1 (ppm) 
(E)-4-methylene-2-phenyl-1-(trimethylsilyl)hept-1-en-3-one (1bb)
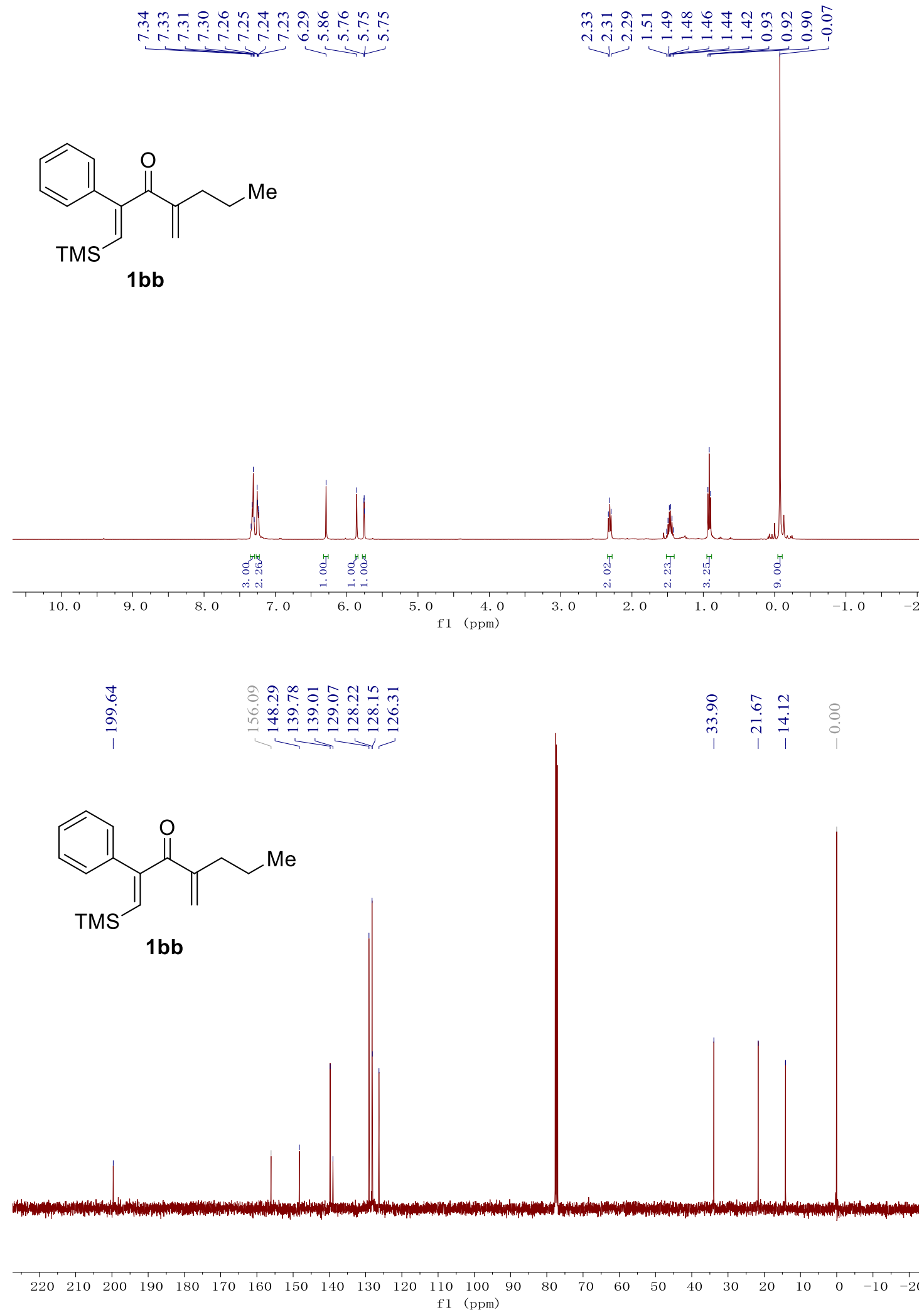
(E)-4-methylene-2-phenyl-1-(trimethylsilyl)dec-1-en-3-one (1bc)
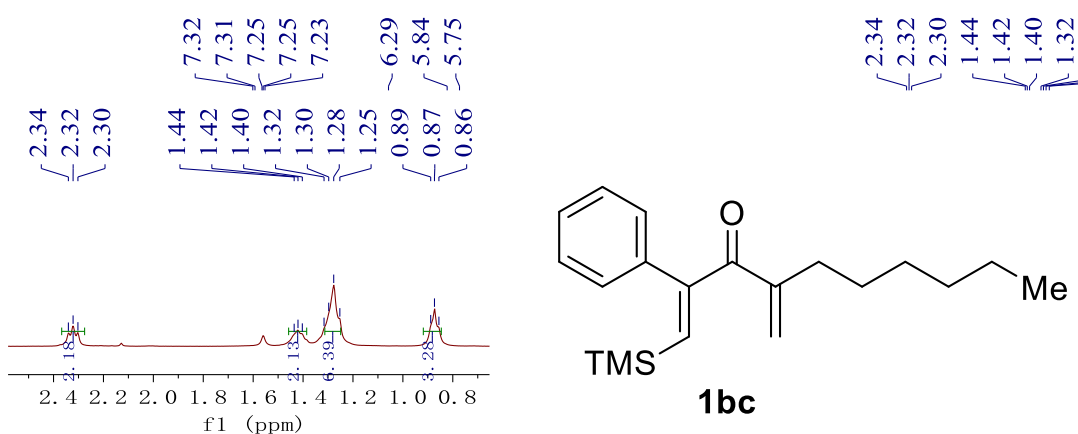

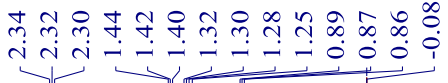

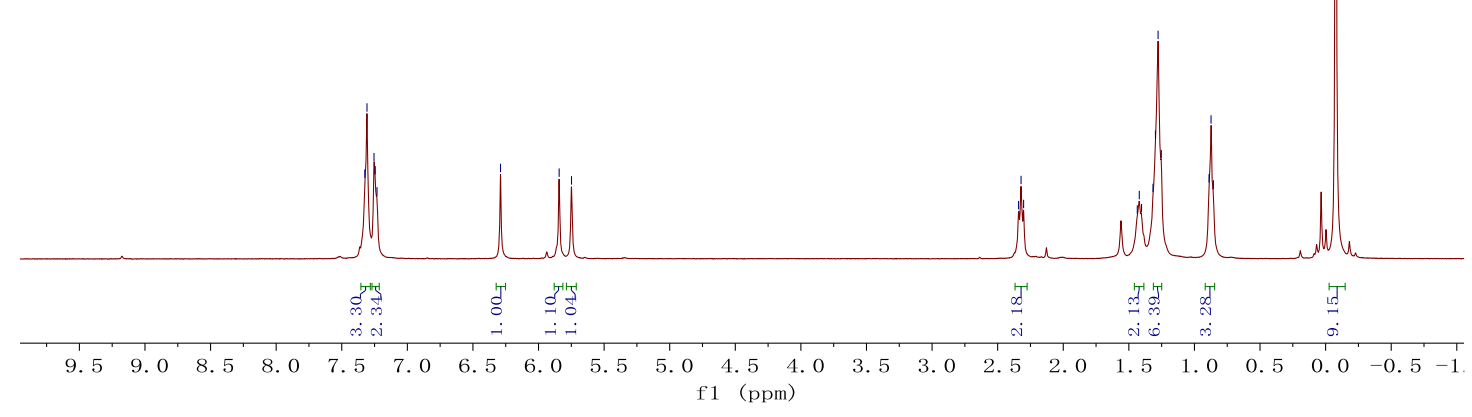

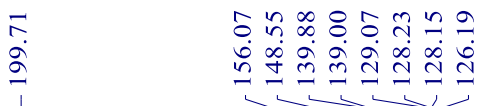

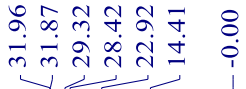<smiles>C=C(CCCCCC)C(=O)C(=C[As])c1ccccc1</smiles>

$\begin{array}{llllllllllllllllllllllllllll}1 & 20 & 210 & 200 & 190 & 180 & 170 & 160 & 150 & 140 & 130 & 120 & 110 & 100 & 90 & 80 & 70 & 60 & 50 & 40 & 30 & 20 & 10 & 0 & -10 & -2\end{array}$ f1 (ppm) 
(E)-5-methyl-4-methylene-2-phenyl-1-(trimethylsilyl)hex-1-en-3-one (1bd)

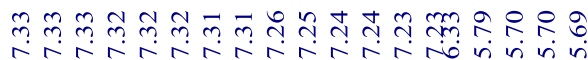

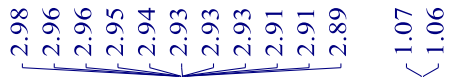<smiles>C=C(C(=O)/C(=C/S(C)(=O)=O)c1ccccc1)C(C)C</smiles>

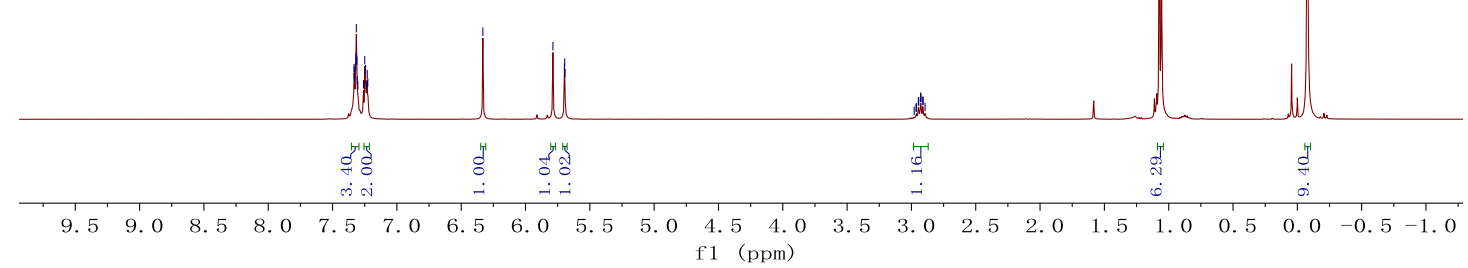

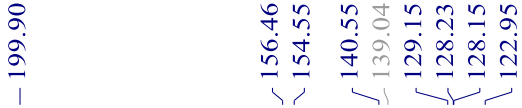

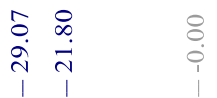<smiles>C=C(C(=O)/C(=C/S(C)(=O)=O)c1ccccc1)C(C)C</smiles>

$1 \mathrm{bd}$ 
<smiles>C=C(C(=O)C(=CS(C)(=O)=O)c1ccccc1)C1CC1</smiles>

1 be
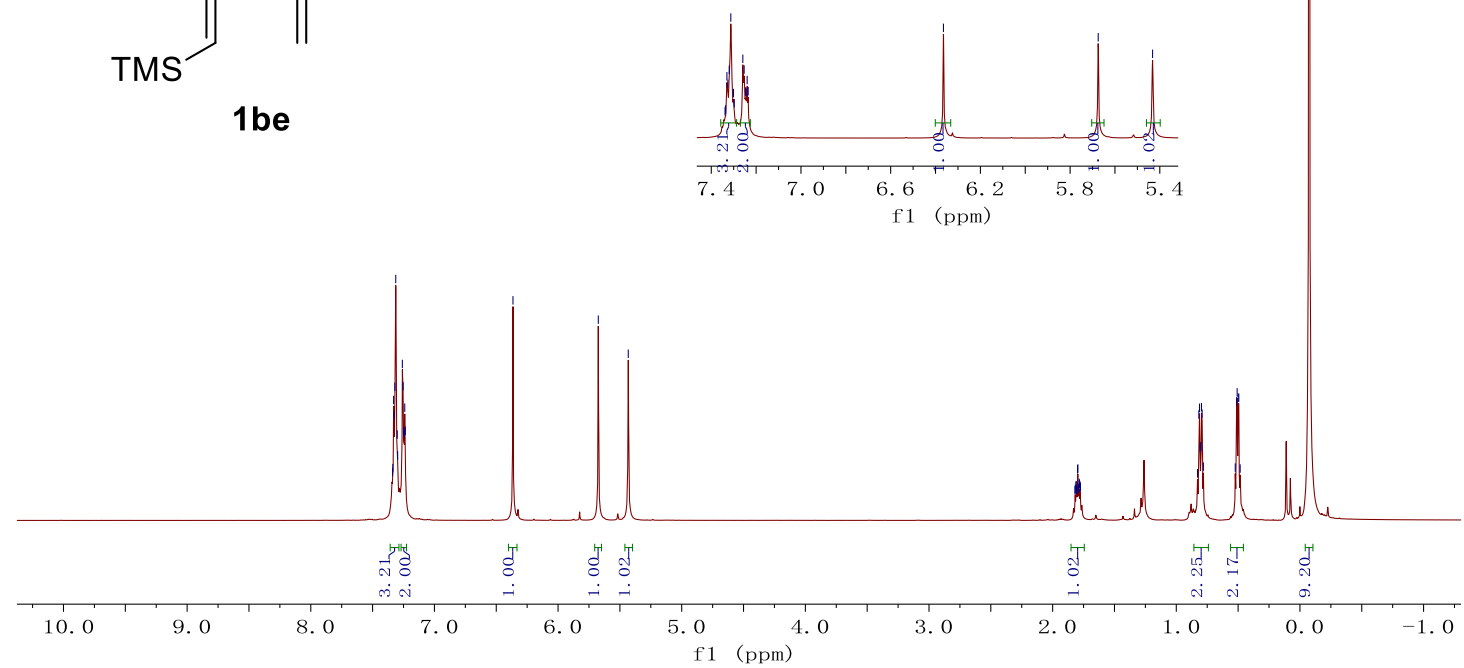

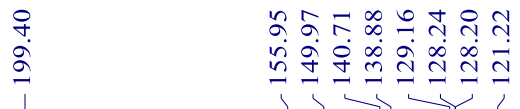

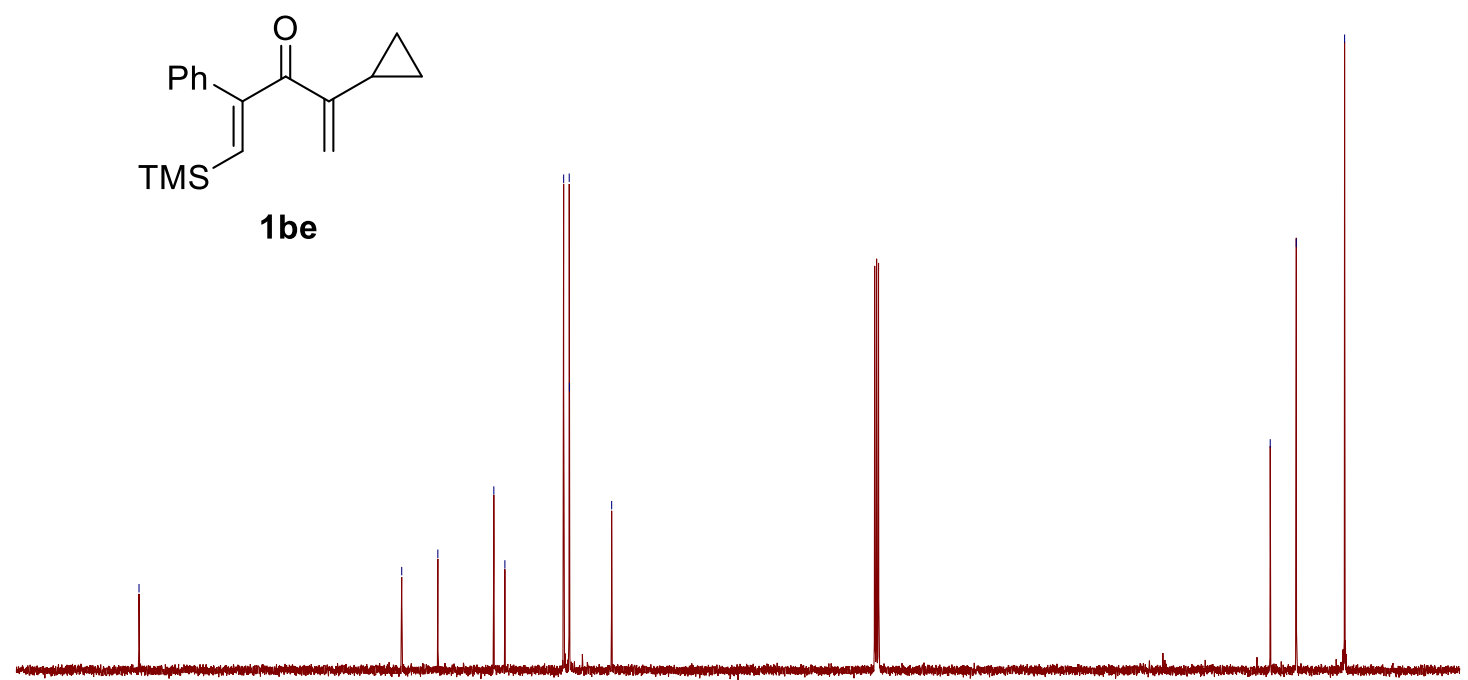

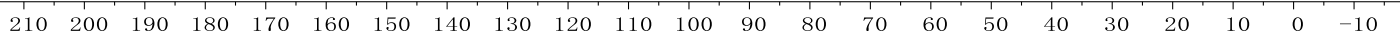


(E)-7-methyl-4-methylene-2-phenyl-1-(trimethylsilyl)octa-1,7-dien-3-one (1bf)

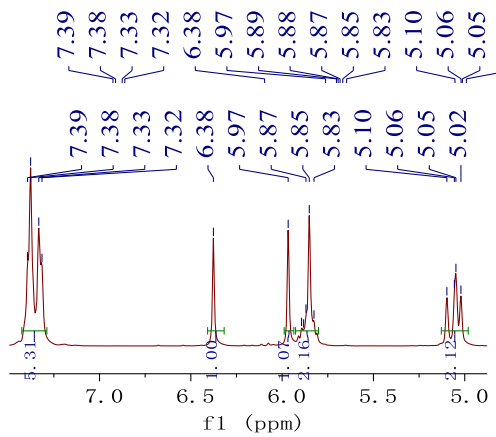

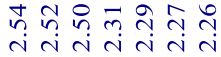

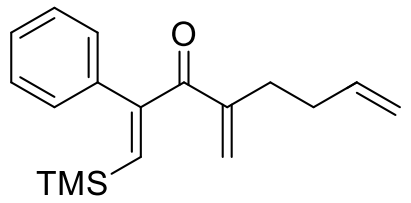

$1 b f$
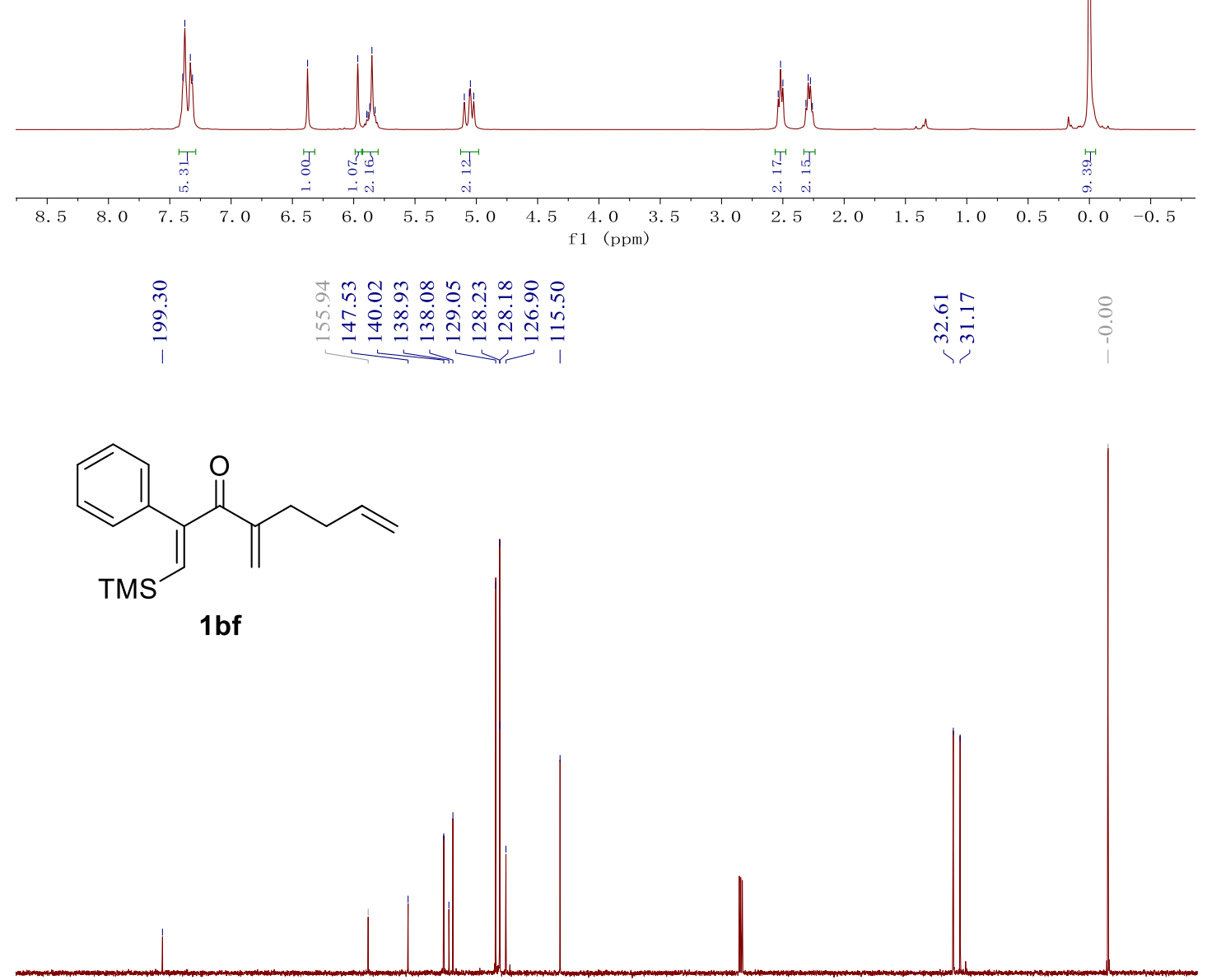

$1 b f$

\begin{tabular}{lllllllllllllllllllllllllllllllll}
\hline 30 & 220 & 210 & 200 & 190 & 180 & 170 & 160 & 150 & 140 & 130 & 120 & 110 & 100 & 90 & 80 & 70 & 60 & 50 & 40 & 30 & 20 & 10 & 0 & -10
\end{tabular}

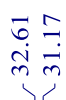

8 f1 (ppm) 
(E)-6-chloro-4-methylene-2-phenyl-1-(trimethylsilyl)hex-1-en-3-one (1bg)

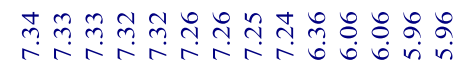

ถే

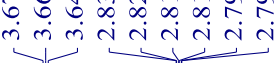

$\underbrace{C}_{T M S}$

1 bg

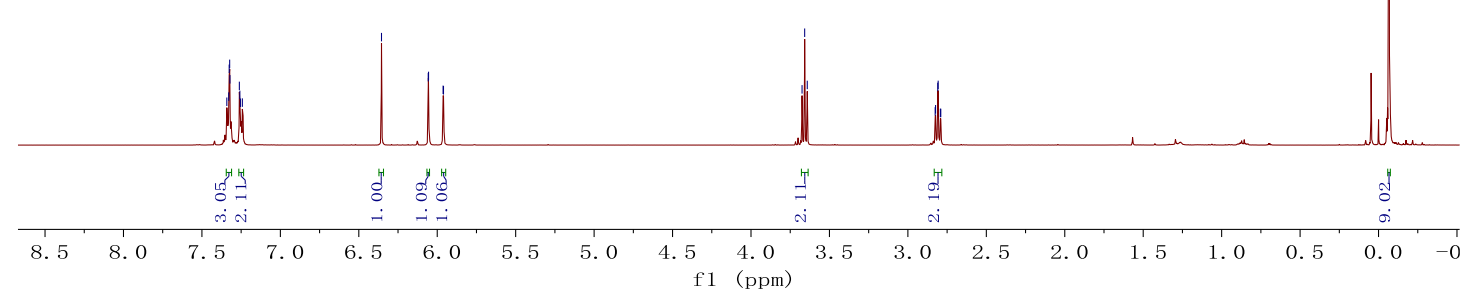

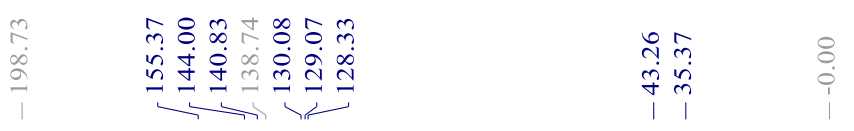<smiles>C=C(CCCl)C(=O)C(=C[S+](C)(=O)=O)c1ccccc1</smiles>

1 bg

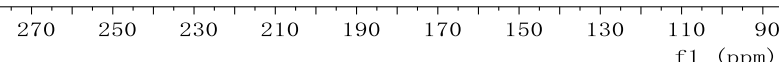


(E)-6-methoxy-4-methylene-2-phenyl-1-(trimethylsilyl)hex-1-en-3-one (1bh)

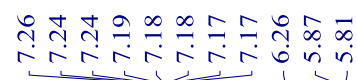

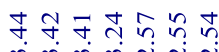

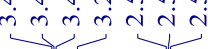<smiles>C=C(CCOC)C(=O)C(=O)C(=CC)c1ccccc1</smiles>

$1 \mathrm{bh}$

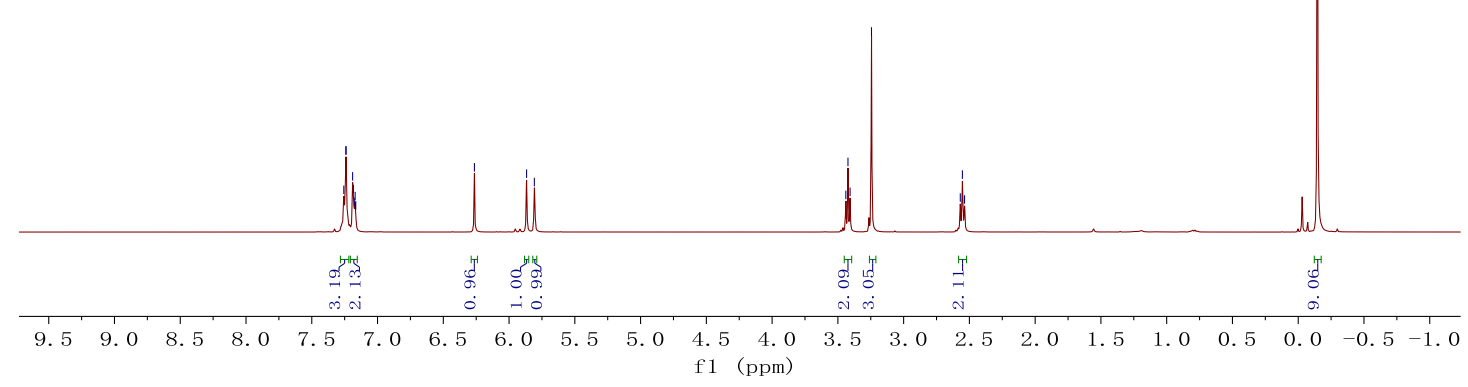

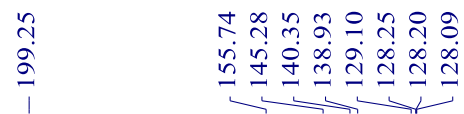<smiles>C=C(CCOC)C(=O)C(=CS)c1ccccc1</smiles>

$1 \mathrm{bh}$

$\begin{array}{lllllllllllllllllllllllllllllll}230 & 220 & 210 & 200 & 190 & 180 & 170 & 160 & 150 & 140 & 130 & 120 & 110 & 100 & 90 & 80 & 70 & 60 & 50 & 40 & 30 & 20 & 10 & 0 & -10 & -20\end{array}$

f1 (ppm)

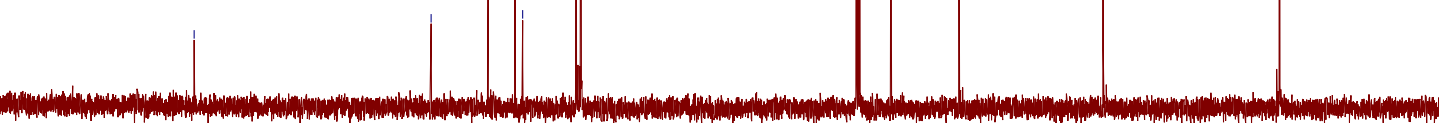




\section{(E)-4-benzyl-2-phenyl-1-(trimethylsilyl)penta-1,4-dien-3-one (1bi)}

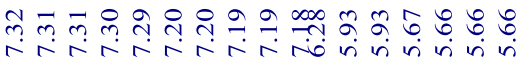

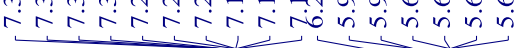

$\underbrace{P h}_{T M S}$

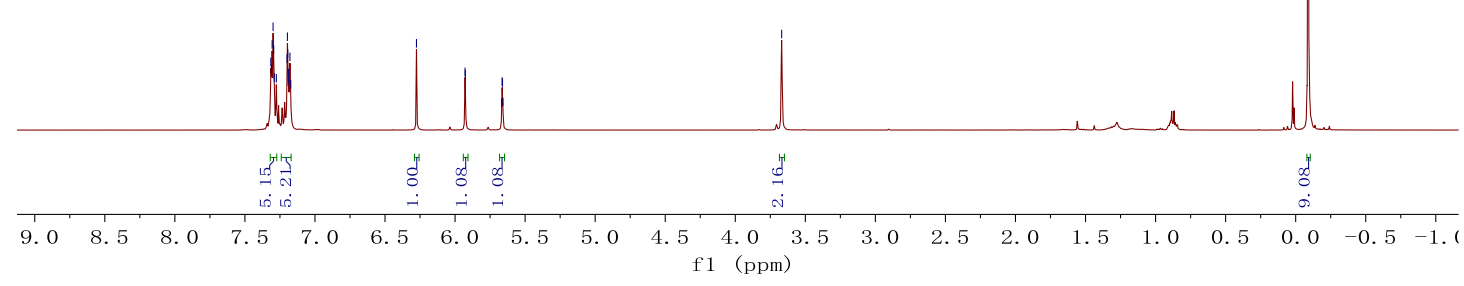

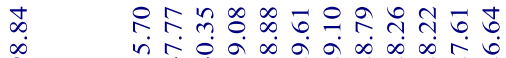

l.

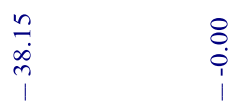<smiles>C=C(Cc1ccccc1)C(=O)C(=CS(C)(=O)=O)c1ccccc1</smiles>

$1 \mathrm{bi}$ 
(E)-2,4-diphenyl-1-(trimethylsilyl)penta-1,4-dien-3-one (1ca)
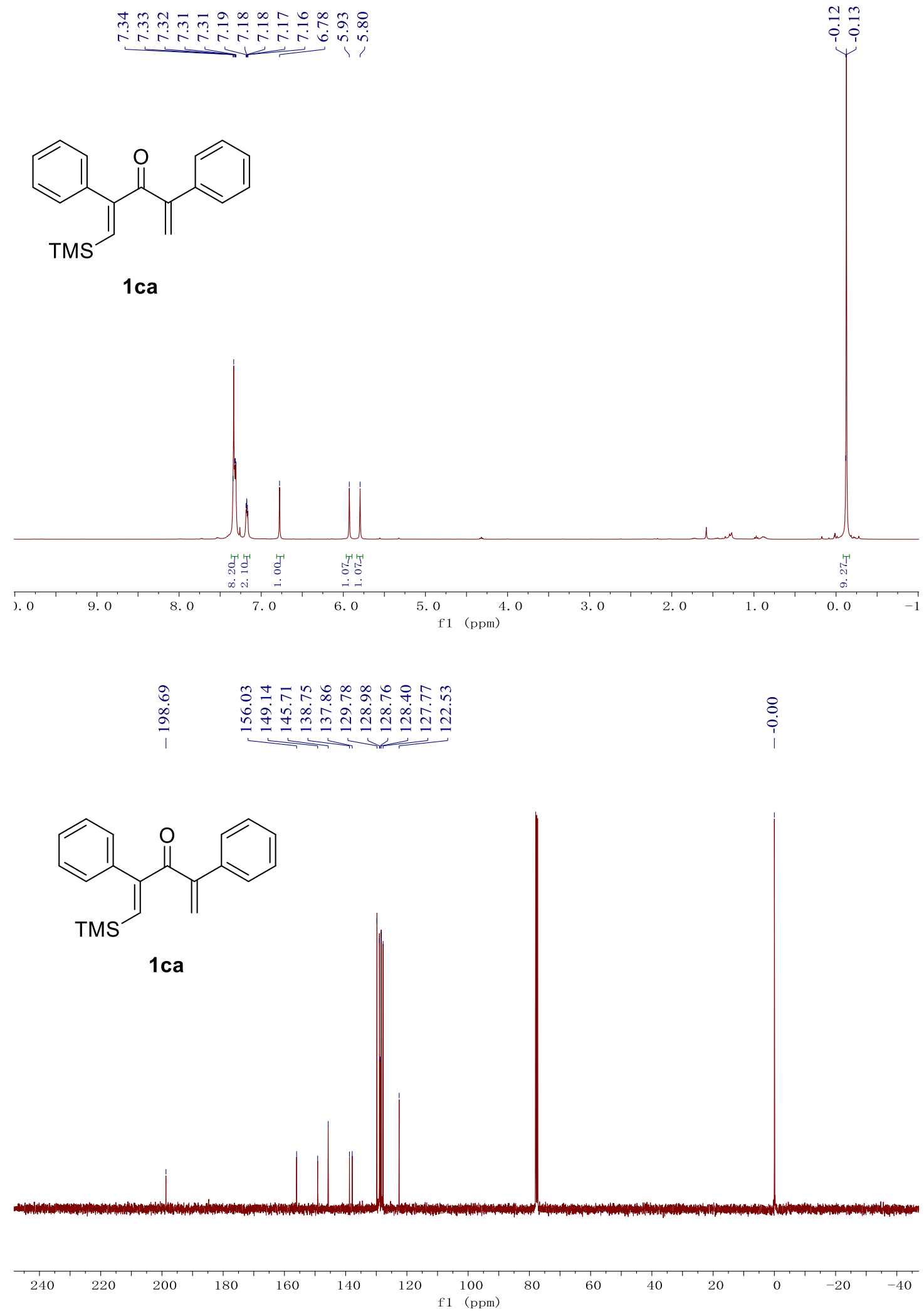


\section{(E)-2-phenyl-4-(o-tolyl)-1-(trimethylsilyl)penta-1,4-dien-3-one (1cb)}

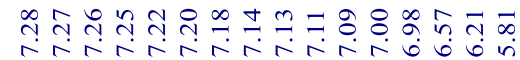<smiles>C=C(C(=O)C(=CS)c1ccccc1)c1ccccc1C</smiles>

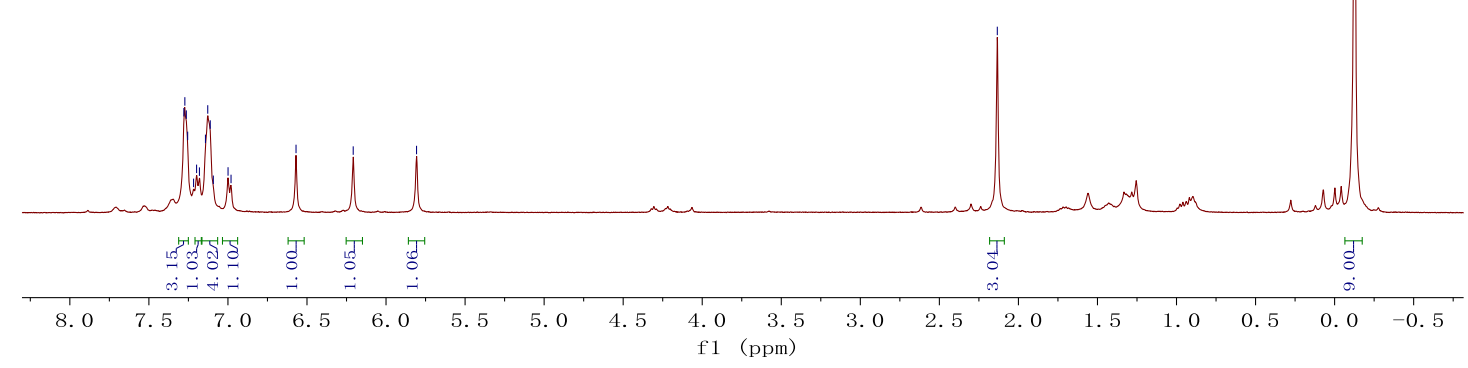

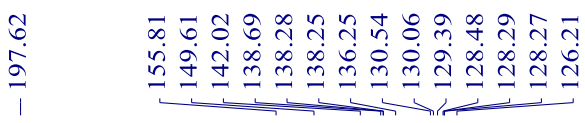

$\begin{array}{ll}0 & \\ 0 & 8 \\ 0 & 0 \\ 1 & 1\end{array}$

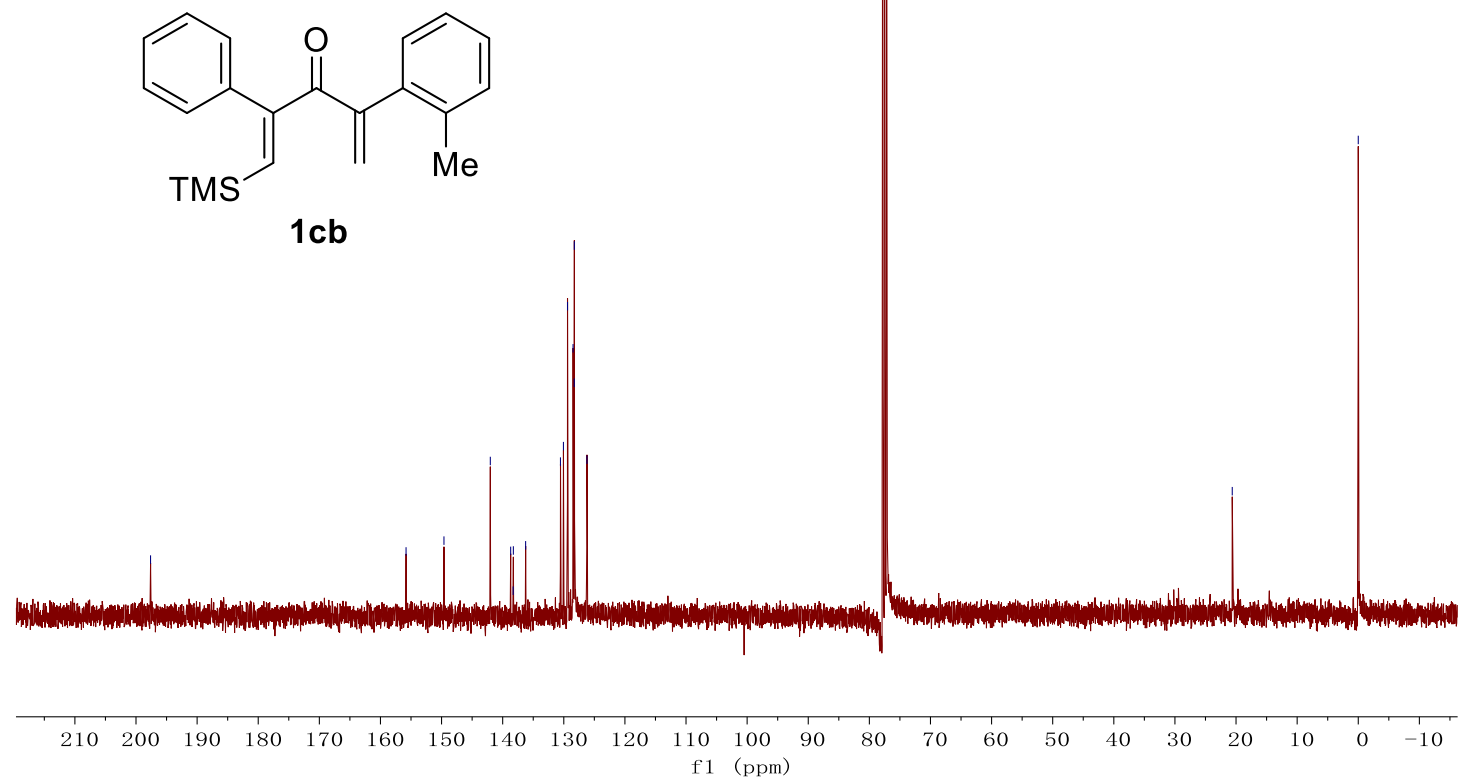




\section{(E)-2-phenyl-4-(thiophen-3-yl)-1-(trimethylsilyl)penta-1,4-dien-3-one (1cc)}

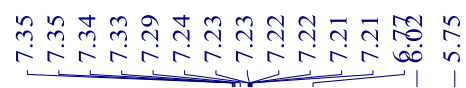

$\begin{array}{lll}\infty & 0 & 0 \\ 0 & 0 & 1 \\ 0 & 0 & 0 \\ 1 & 1 & 1\end{array}$<smiles>C=C(C(=O)c1ccsc1)/C(=C\C(C)(C)C)c1ccccc1</smiles>
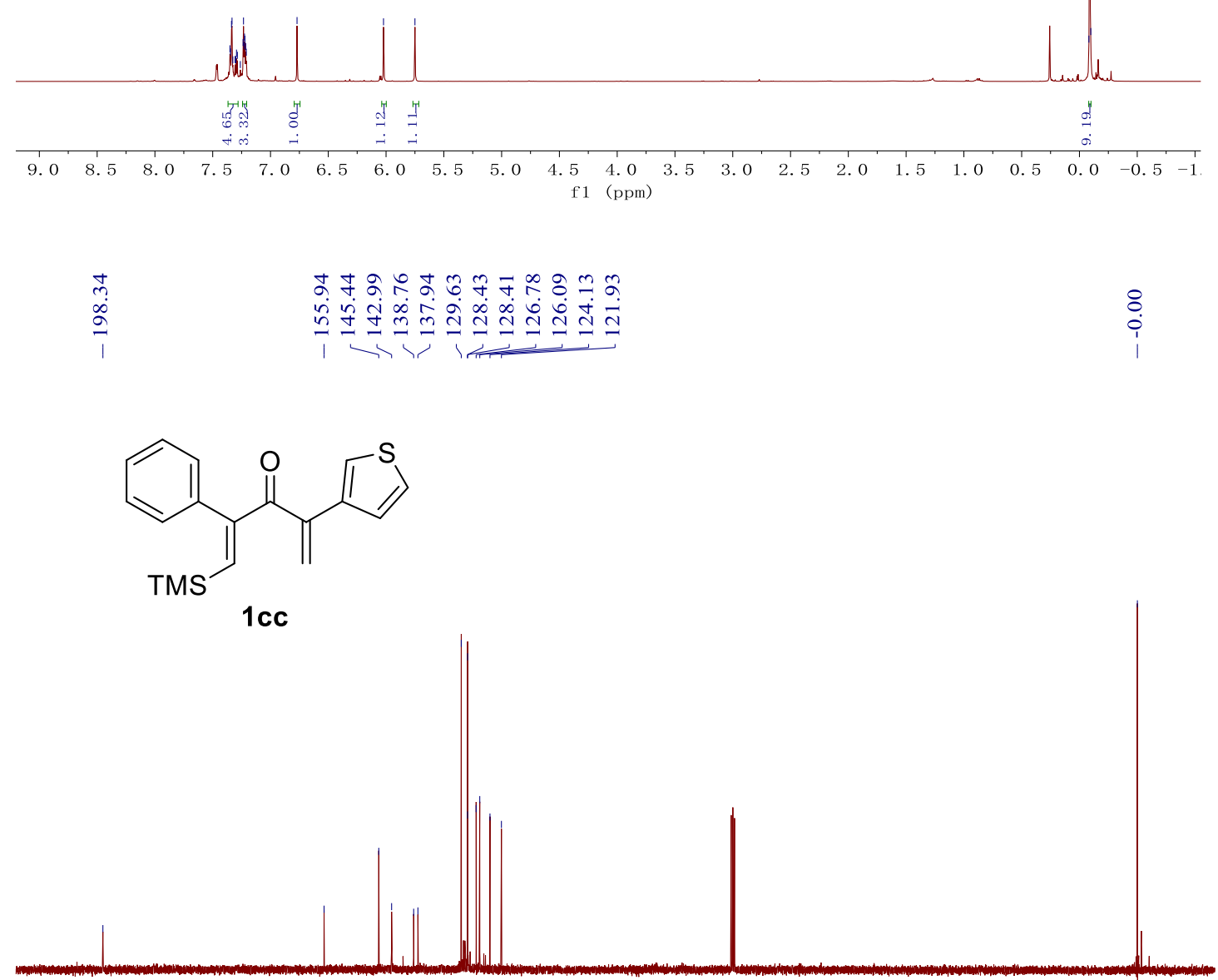

$\begin{array}{lllllllllllllllllllllllll} & 1 \\ 210 & 200 & 190 & 180 & 170 & 160 & 150 & 140 & 130 & 120 & 110 & 100 & 90 & 80 & 70 & 60 & 50 & 40 & 30 & 20 & 10 & 0 & -10\end{array}$ f1 (ppm) 
(E)-2-benzyl-4-methyl-1-(trimethylsilyl)penta-1,4-dien-3-one (1da)

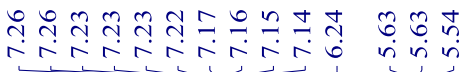

$\stackrel{\infty}{\infty}$
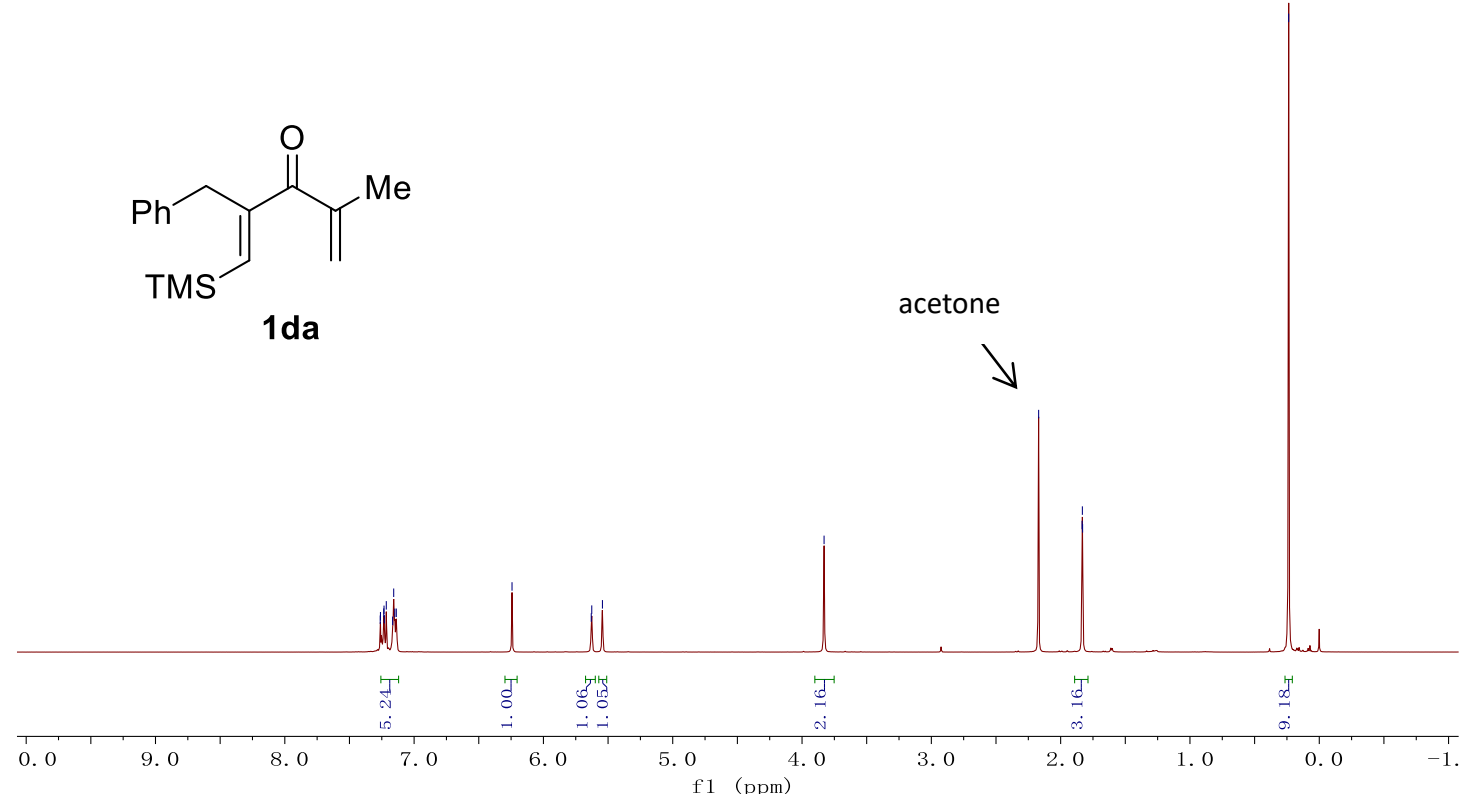

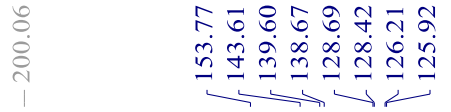

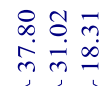

f1 (ppm)<smiles>C=C(C)C(=O)/C(=C/C)Cc1ccccc1</smiles>

$1 \mathrm{da}$

$250 \quad 230 \quad 210 \quad 190 \quad 170 \quad 150$

110
1

acetone

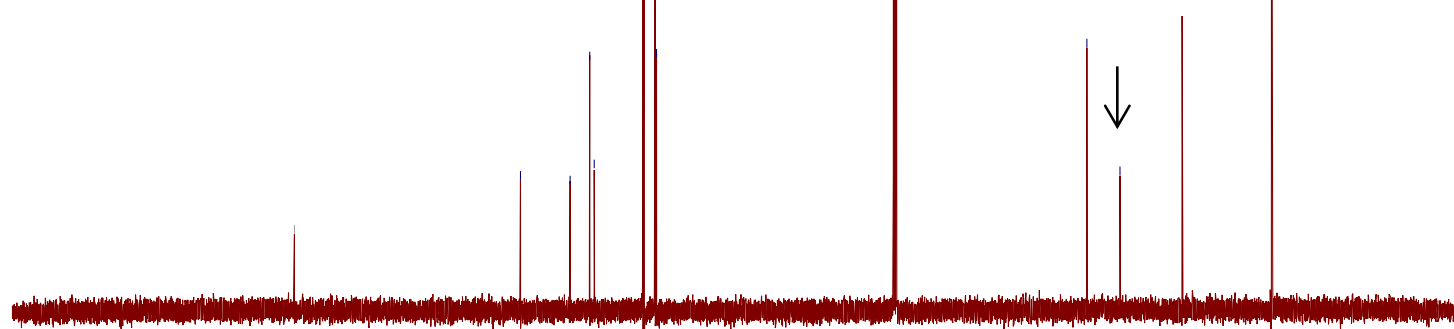




\section{(E)-2-methyl-4-((trimethylsilyl)methylene)oct-1-en-3-one (1db)}

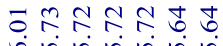

in in

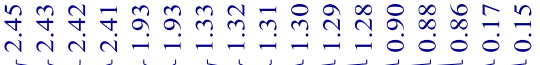<smiles>C=C(C)C(=O)C(=CS)CCCC</smiles>

$1 \mathrm{db}$

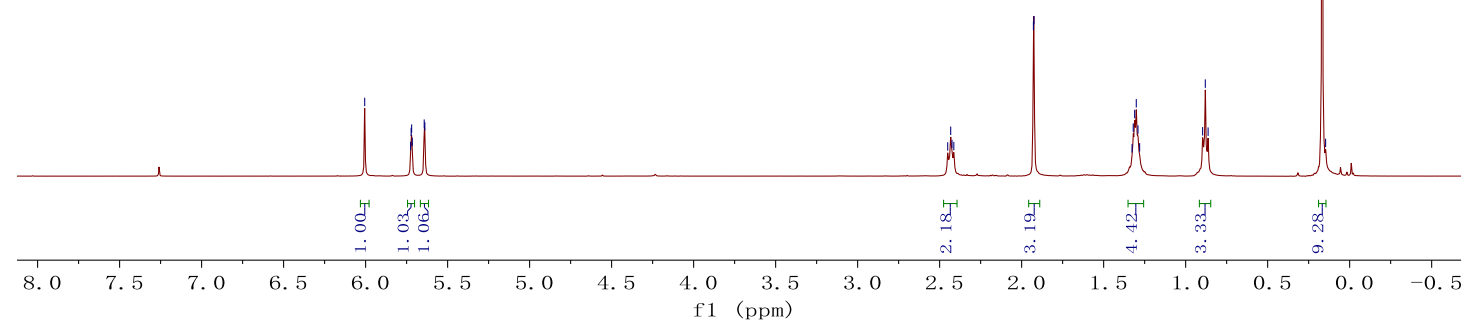

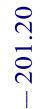
둥ํำ
$\operatorname{lin}$

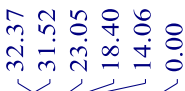

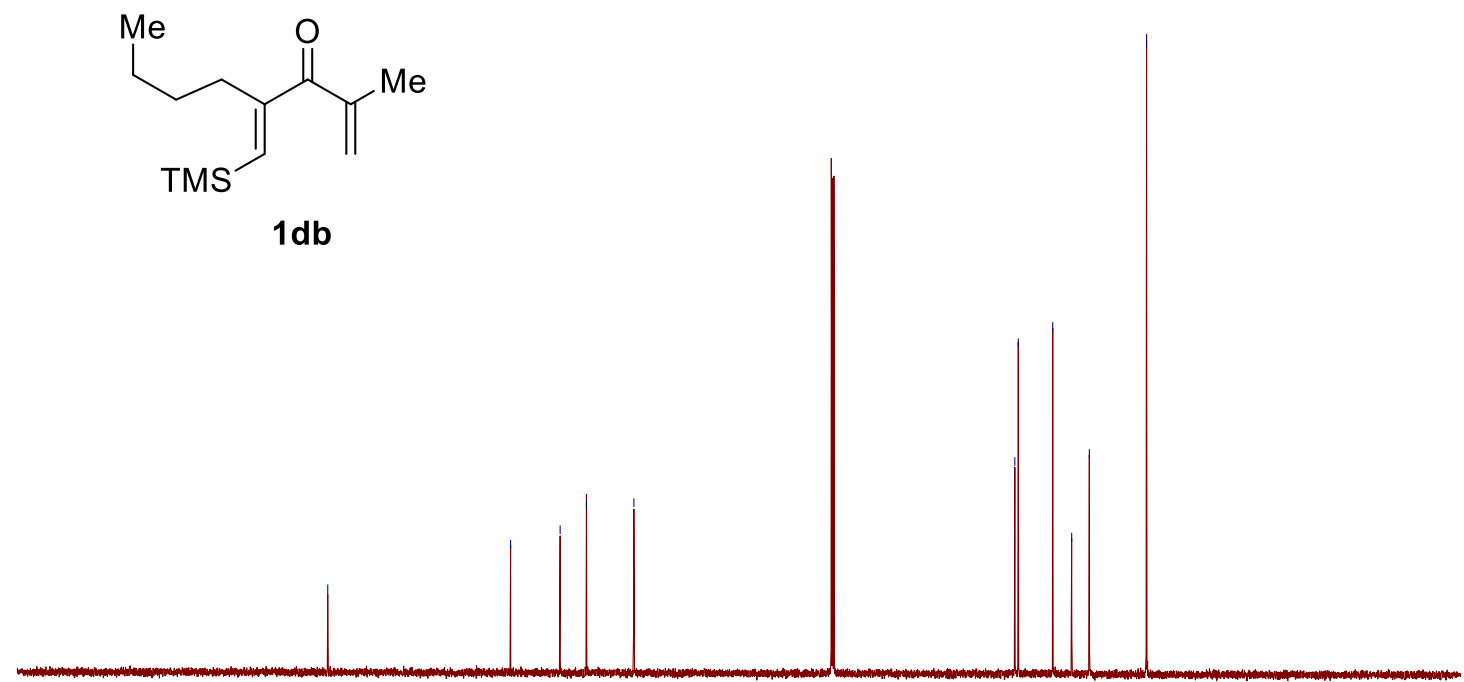

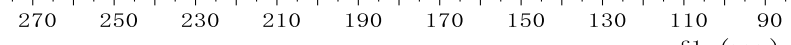


(E)-2,5-dimethyl-4-((trimethylsilyl)methylene)hex-1-en-3-one (1dc)

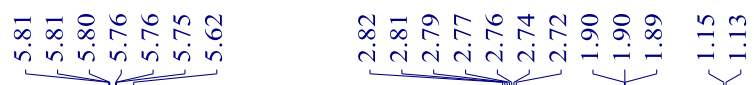

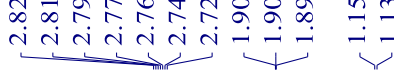<smiles>C=C(C)C(=O)C(=CS(C)(C)(C)(C)C)C(C)C</smiles>

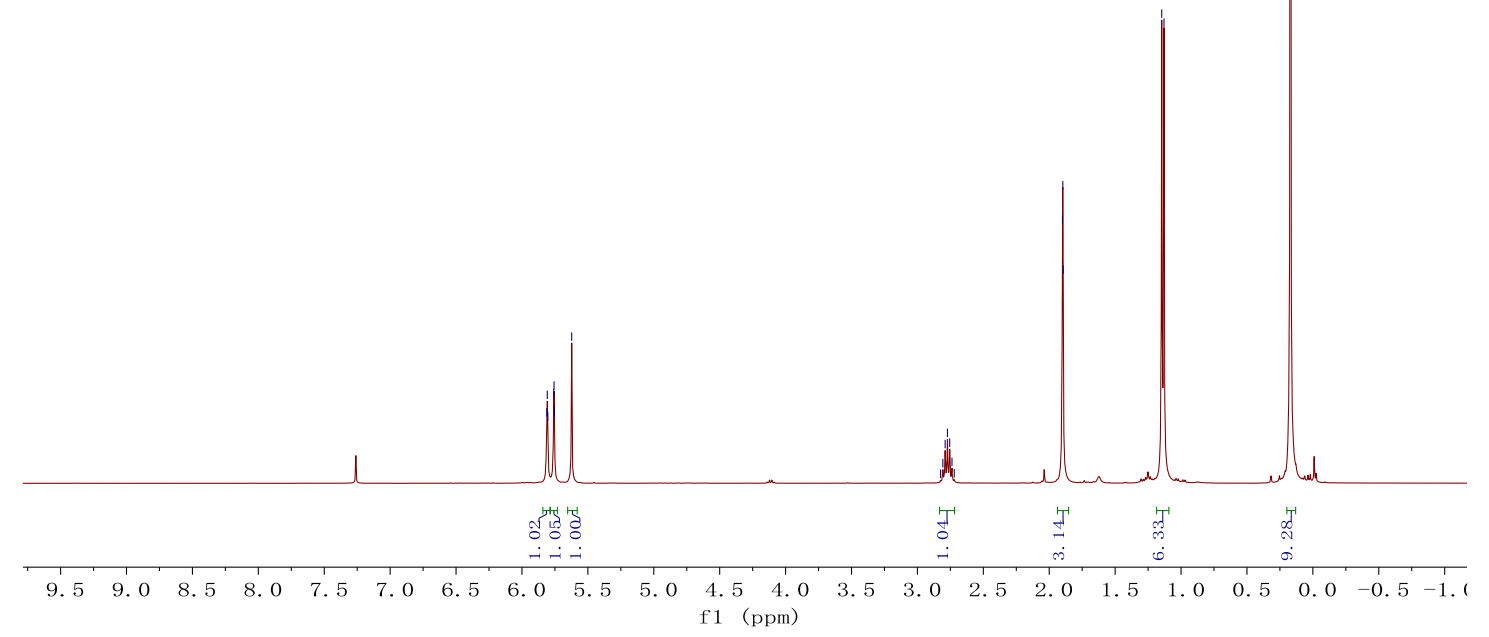

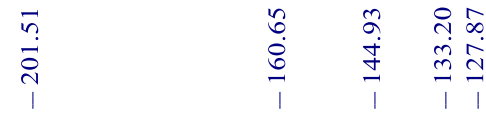

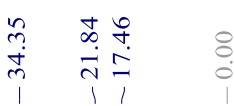<smiles>C=C(C)C(=O)C(=CC(C)C)C(C)C</smiles>

$1 d c$

$\begin{array}{lllllllllllll}210 & 200 & 190 & 180 & 170 & 160 & 150 & 140 & 130 & 120 & 110 & 100 & 90\end{array}$ f1 (ppm) 


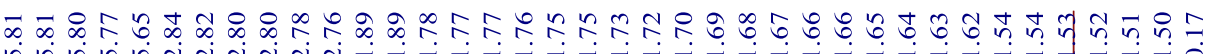

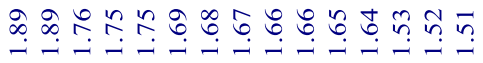

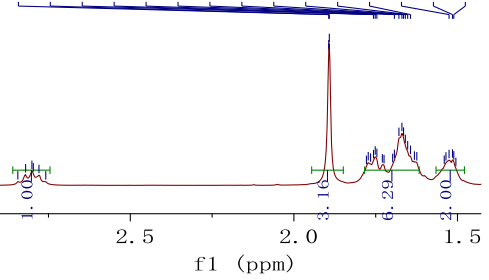<smiles>C=C(C)C(=O)/C(=C/S(C)(=O)=O)C1CCCC1</smiles>

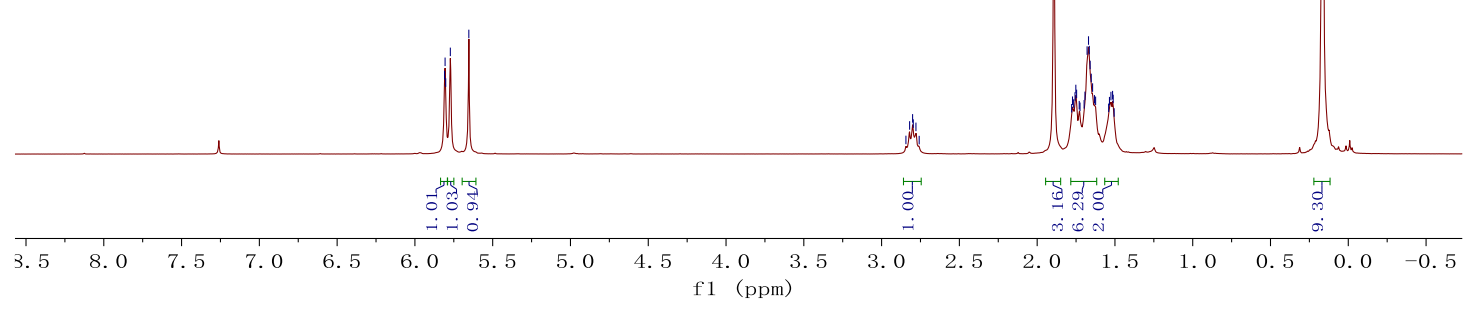

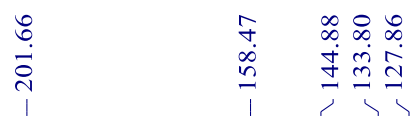

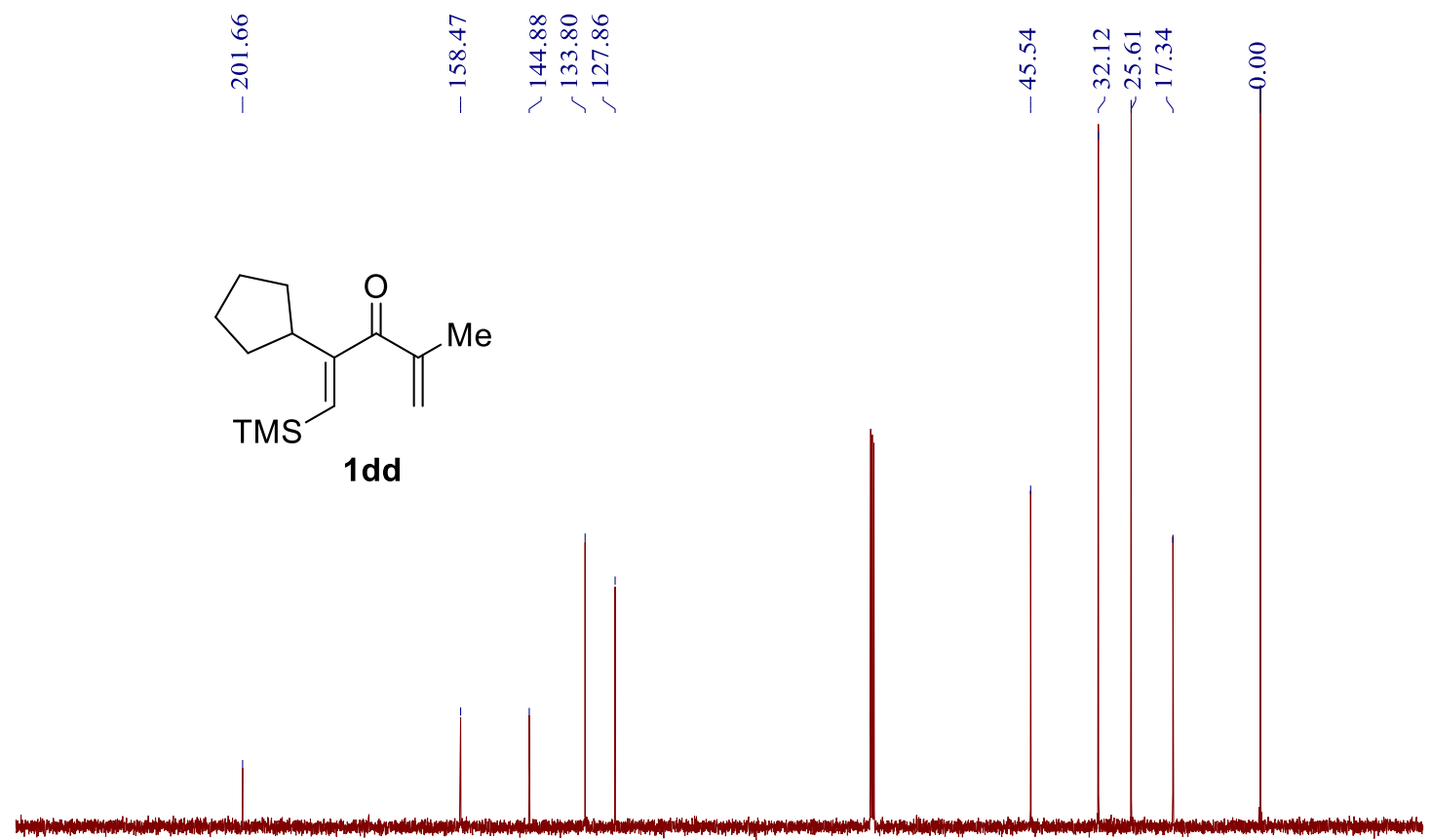
f1 $(\mathrm{ppm})$ 
<smiles>C=C(C)C(=O)C(=CC(C)(C)C)C1CCCCC1</smiles>
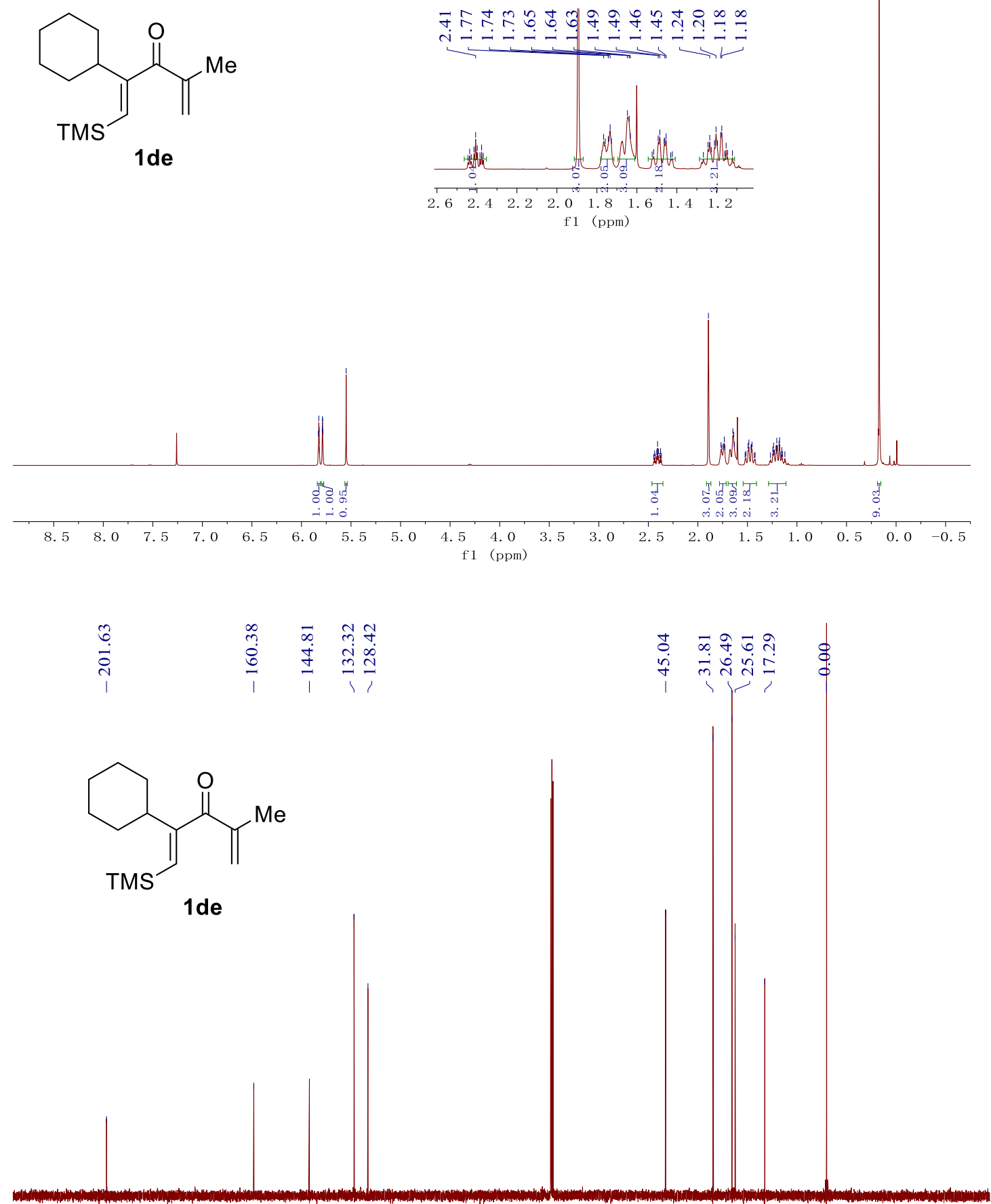


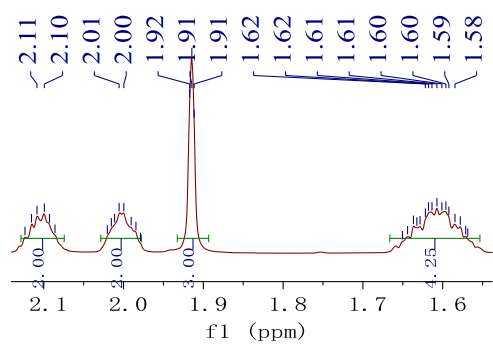<smiles>C=C(C)C(=O)C(=CC(C)(C)C)C1=CCCCC1</smiles>

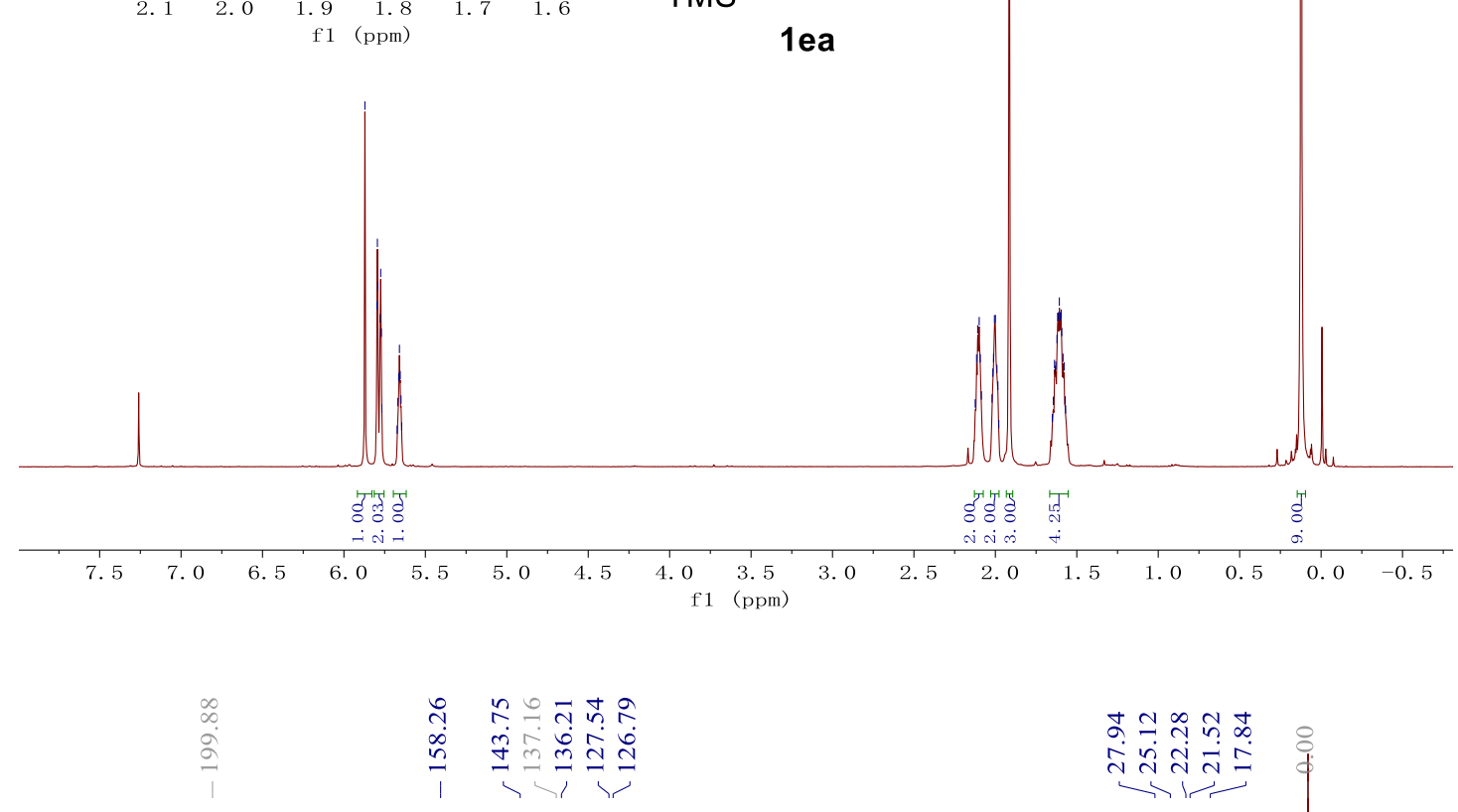

1ea

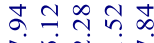

$\hat{n} \sin$

$\mathrm{M}$

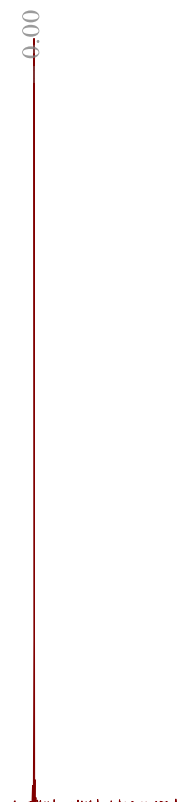


(E)-2-methyl-4-((trimethylsilyl)methylene)dodec-1-en-5-yn-3-one (1fa)

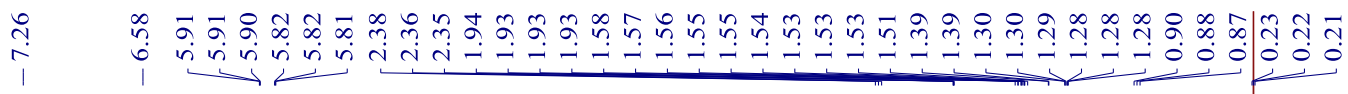

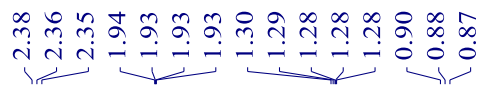
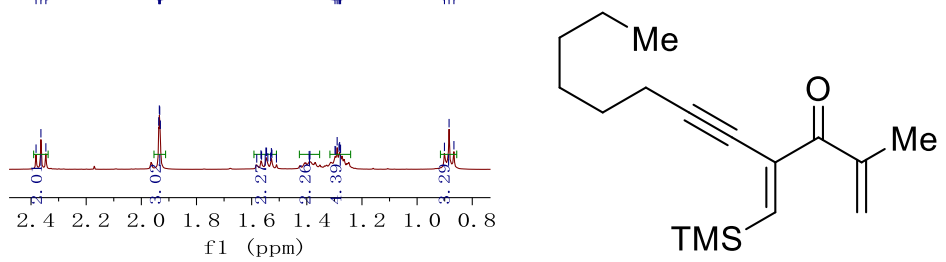

$1 \mathrm{fa}$

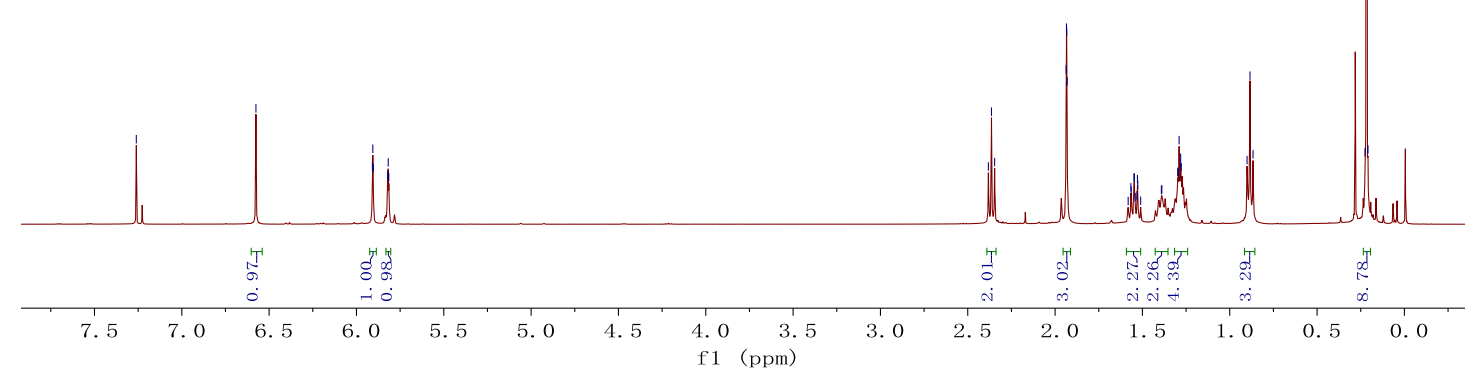

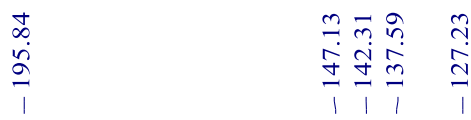

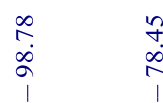

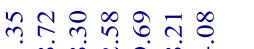

$\bar{m} \stackrel{\infty}{\infty} \underset{\infty}{\infty} \dot{\sim}$

i 1<smiles>C=C(C)C(=O)C(C#CCCCCCC)=CCS(C)(=O)=O</smiles>

1 fa

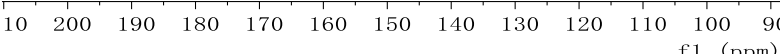


(1E,4E)-4-methyl-2-phenyl-1-(trimethylsilyl)hexa-1,4-dien-3-one (1ga)

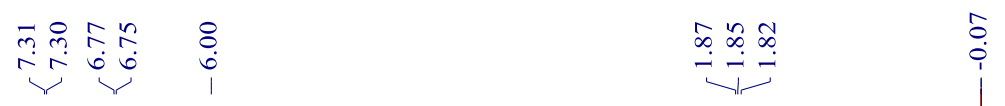<smiles>CC=C(C)C(=O)C(=C[S+](C)(=O)=O)c1ccccc1</smiles>

1 ga
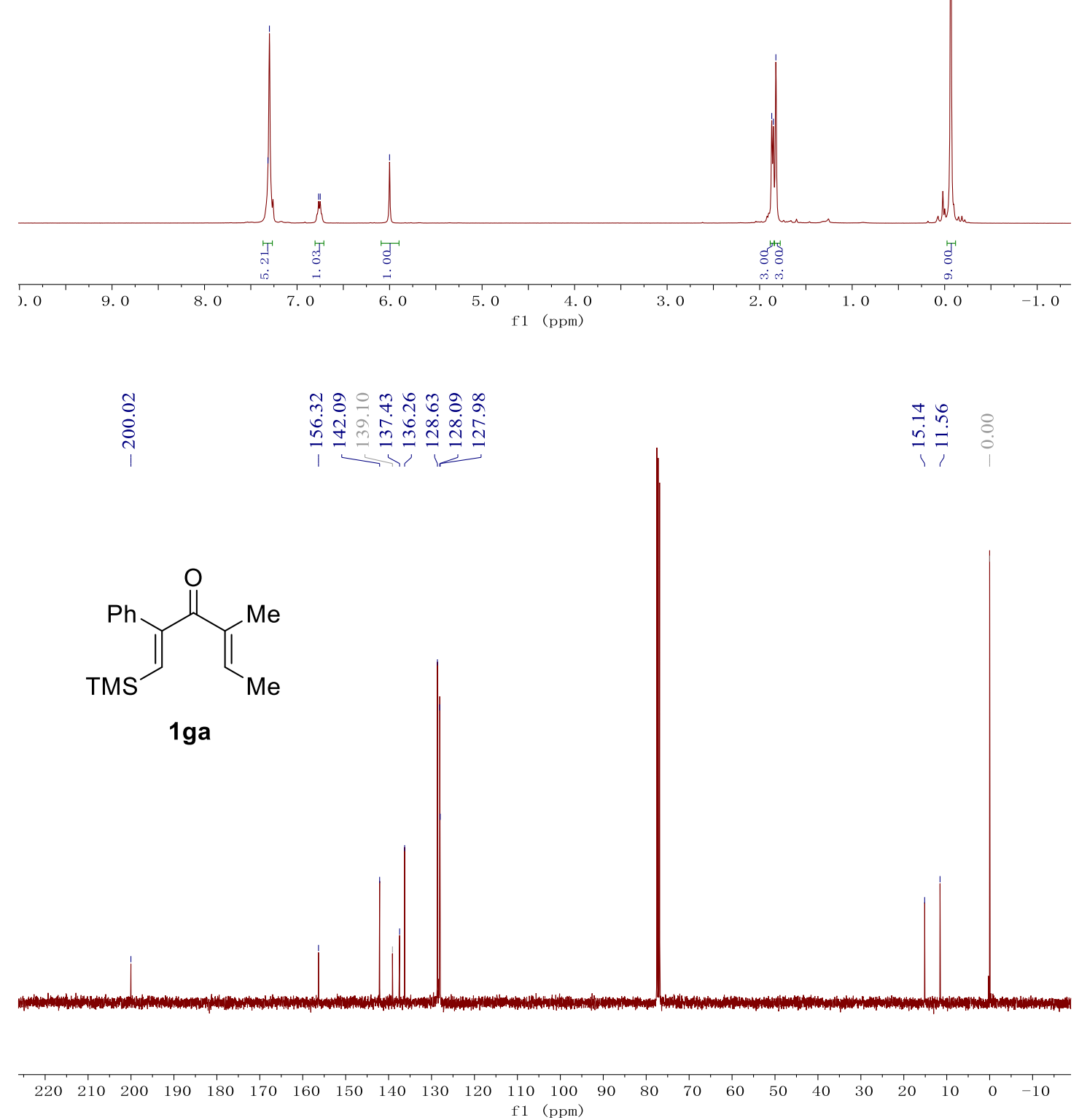
(1E,4E)-4-isopropyl-2-phenyl-1-(trimethylsilyl)hexa-1,4-dien-3-one (1gb)

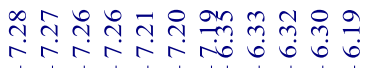

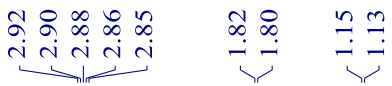<smiles>C/C=C(/C(=O)/C(=C/S(C)(=O)=O)c1ccccc1)C(C)C</smiles>

$1 g b$

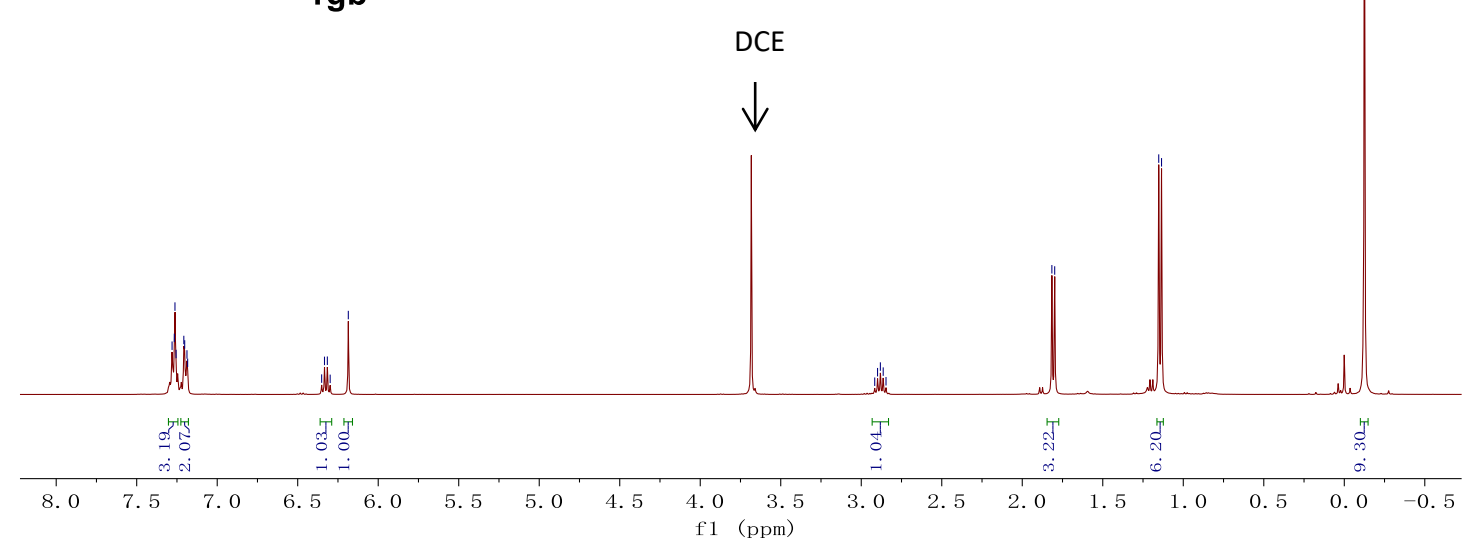

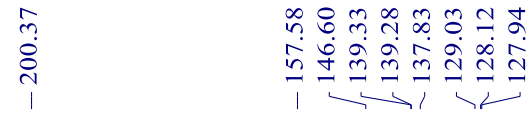

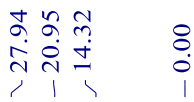

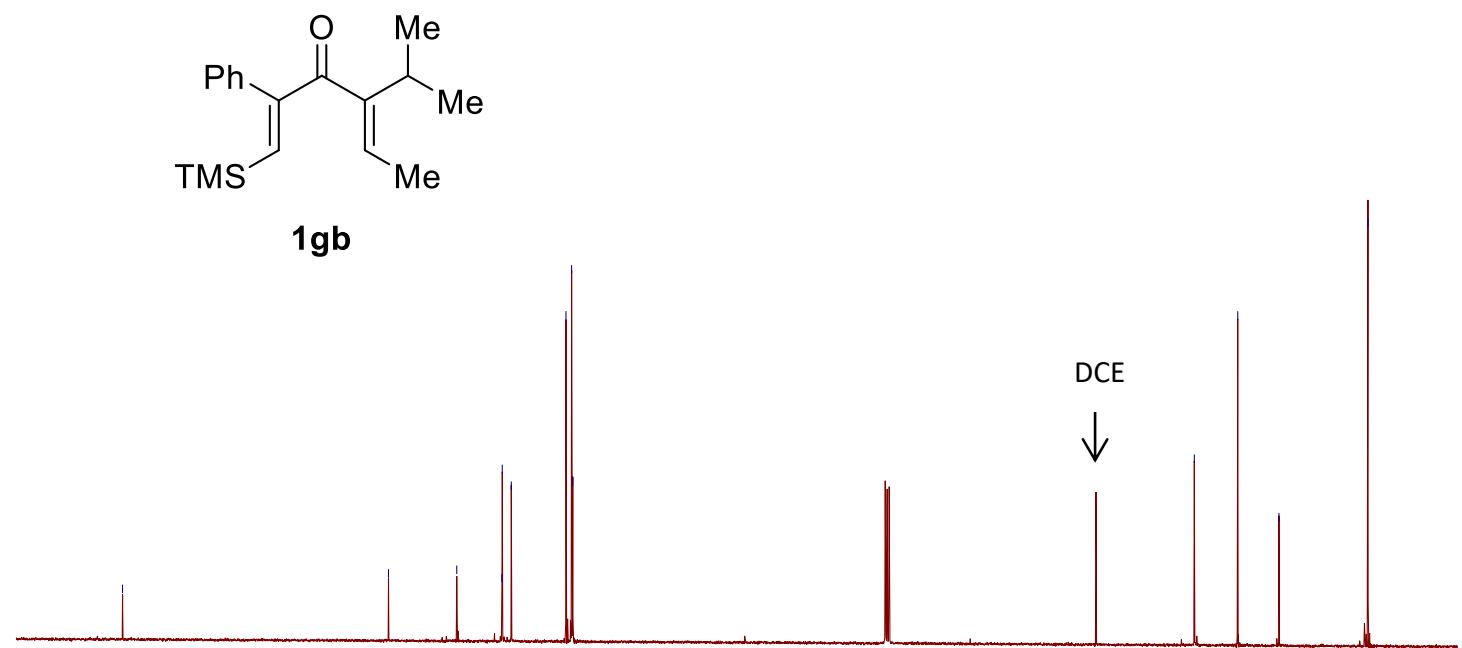

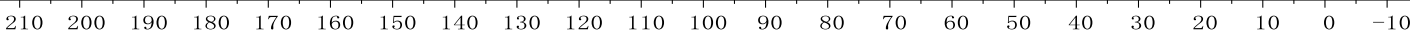
f1 (ppm) 
(1E,4E)-2-methyl-1,4-diphenyl-5-(trimethylsilyl)penta-1,4-dien-3-one (1gc)

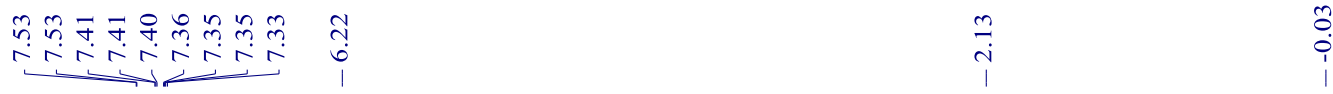<smiles>CC(=Cc1ccccc1)C(=O)C(=CS(C)(=O)=O)c1ccccc1</smiles>

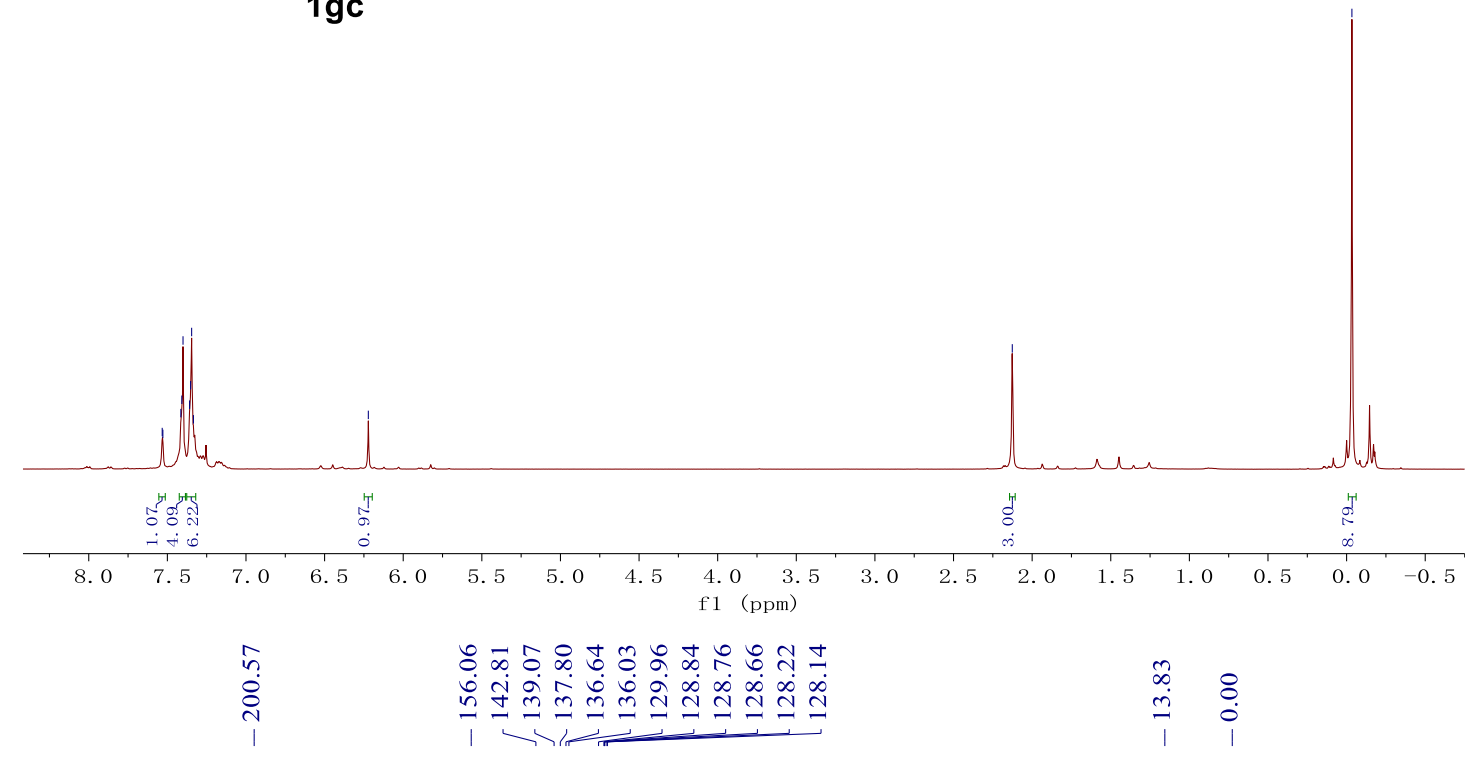<smiles>CC(=Cc1ccccc1)C(=O)C(=C[S+](C)(=O)=O)c1ccccc1</smiles>

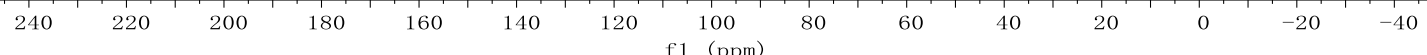
f1 (ppm) 


\section{(E)-4-methyl-1,2-diphenyl-1-(trimethylsilyl)penta-1,4-dien-3-one (1ha)}

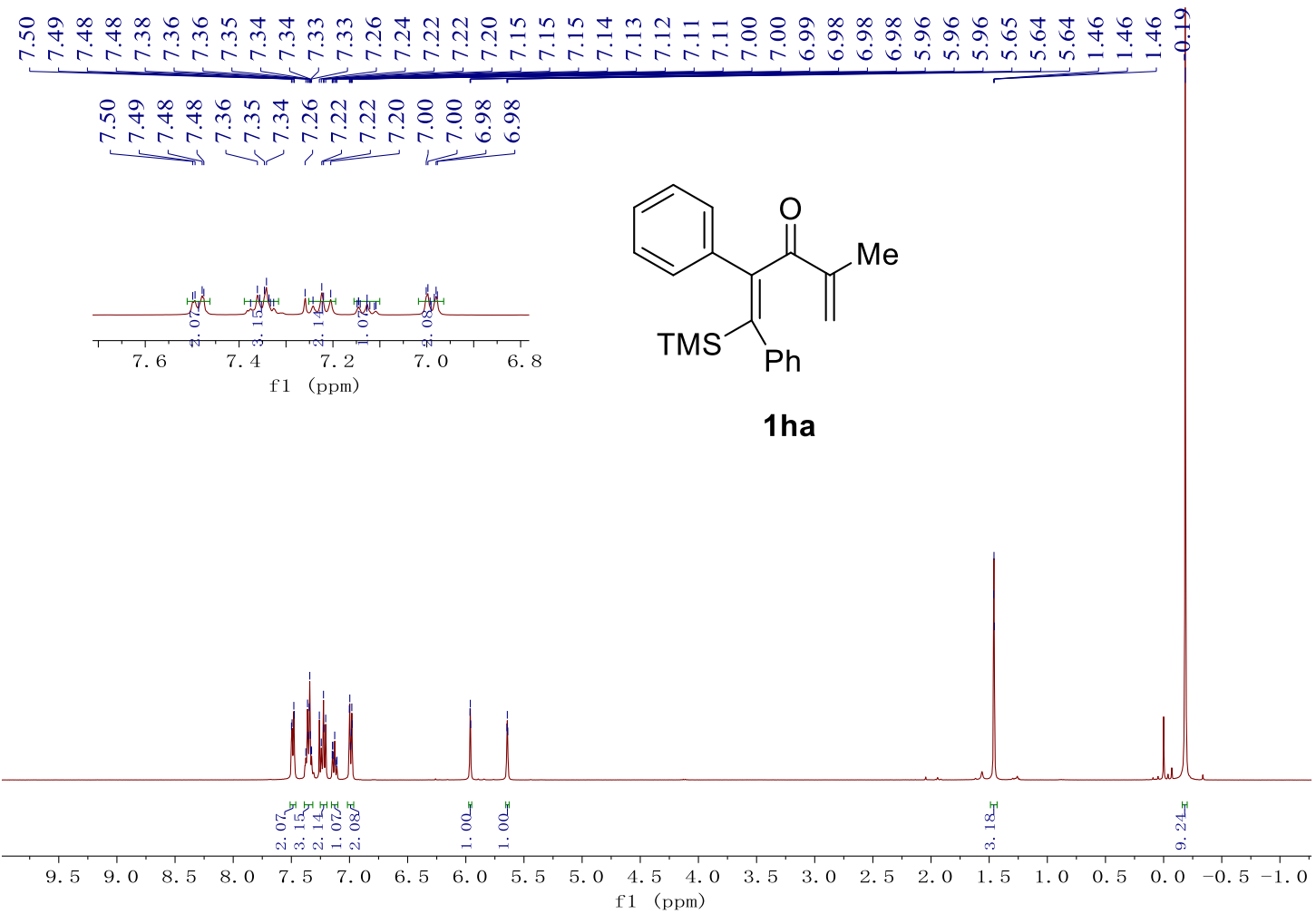

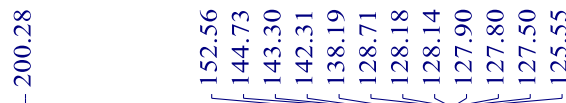

$\therefore \rightarrow \pm 8 \otimes$ in

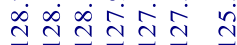

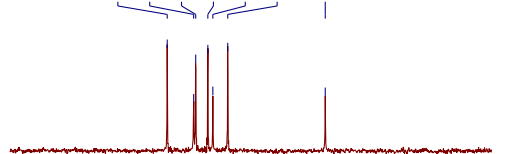

$\begin{array}{llllllllll}131 & 130 & 129 & 128 & 127 & 126 & 125 & 124 & 123\end{array}$

f1 (ppm)

$\begin{array}{ll}\hat{0} & 8 \\ 0 & 0 \\ 1 & 1\end{array}$
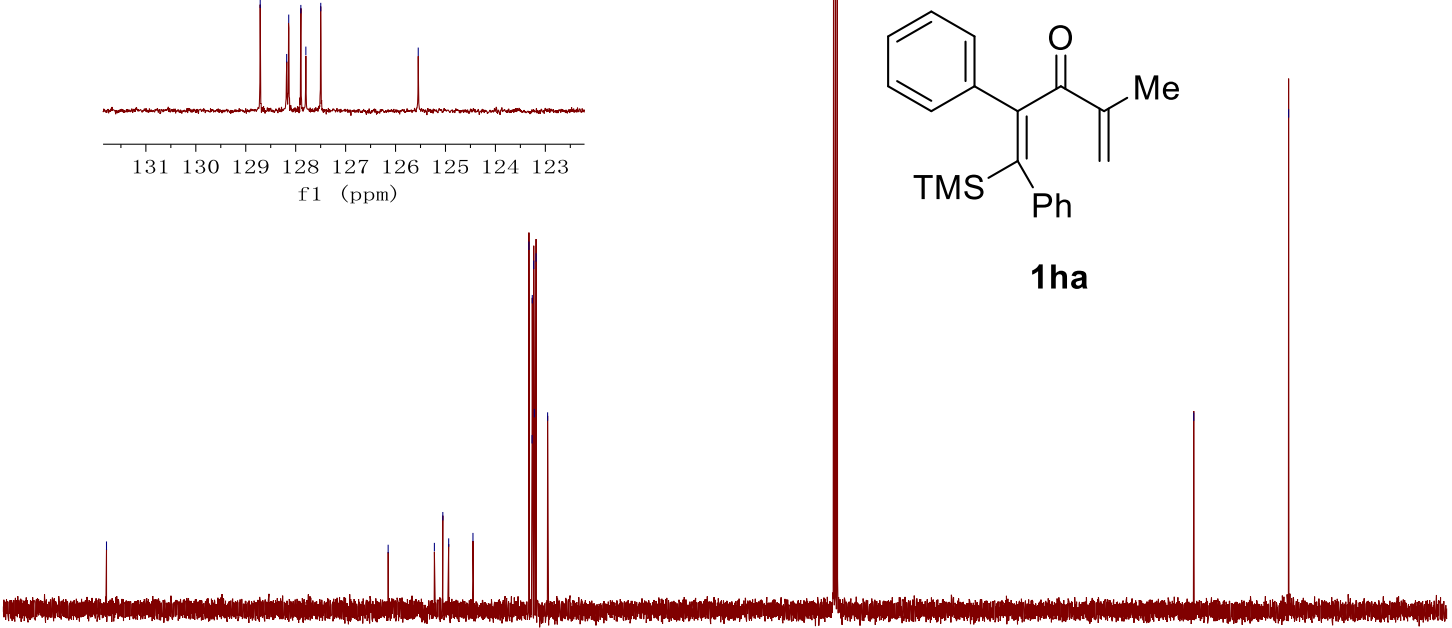

$\begin{array}{llllllllllllr}210 & 200 & 190 & 180 & 170 & 160 & 150 & 140 & 130 & 120 & 110 & 100 & 90\end{array}$ 
(E)-4,5-dimethyl-2-phenyl-1-(trimethylsilyl)hexa-1,4-dien-3-one (1ia)

$$
\underbrace{\frac{1}{\infty}+\frac{1}{2}}
$$

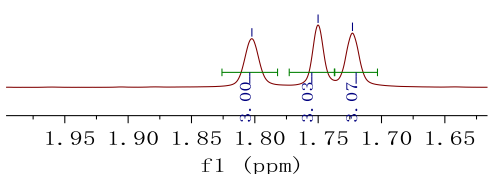
f1 (ppm)

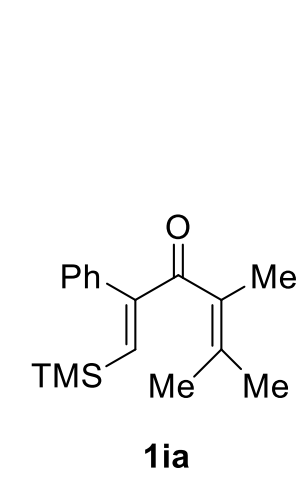

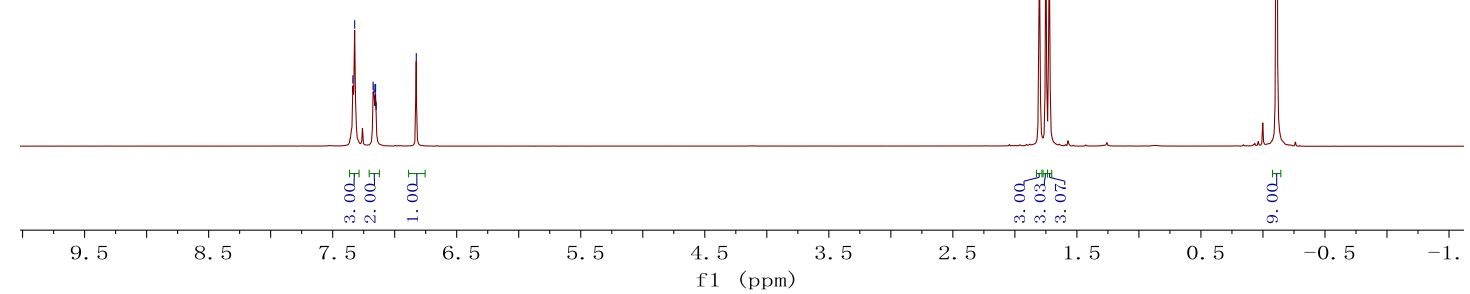

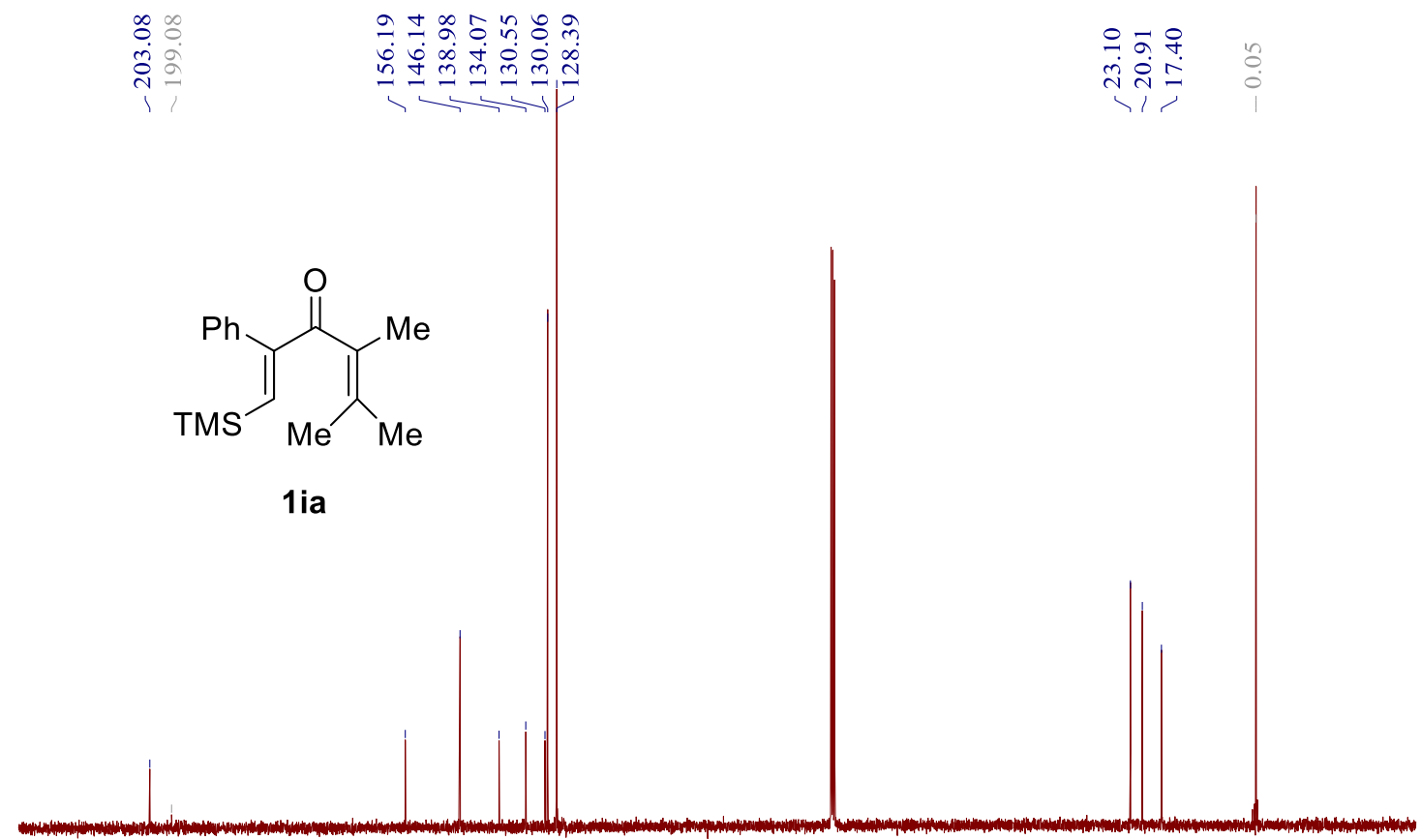

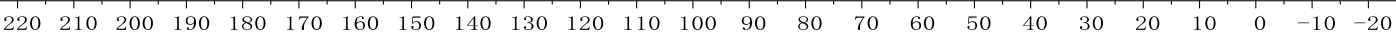
f1 (ppm) 
(E)-4-methyl-2-phenyl-1-(triethylsilyl)penta-1,4-dien-3-one (1ja)
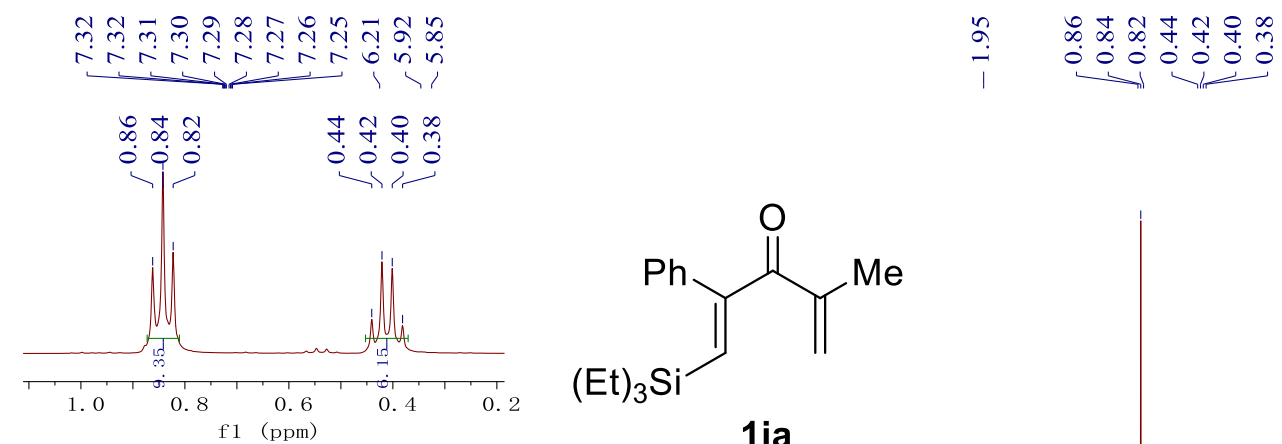<smiles>C=C(C)C(=O)/C(=C/[SiH2]C(C)C)c1ccccc1</smiles>
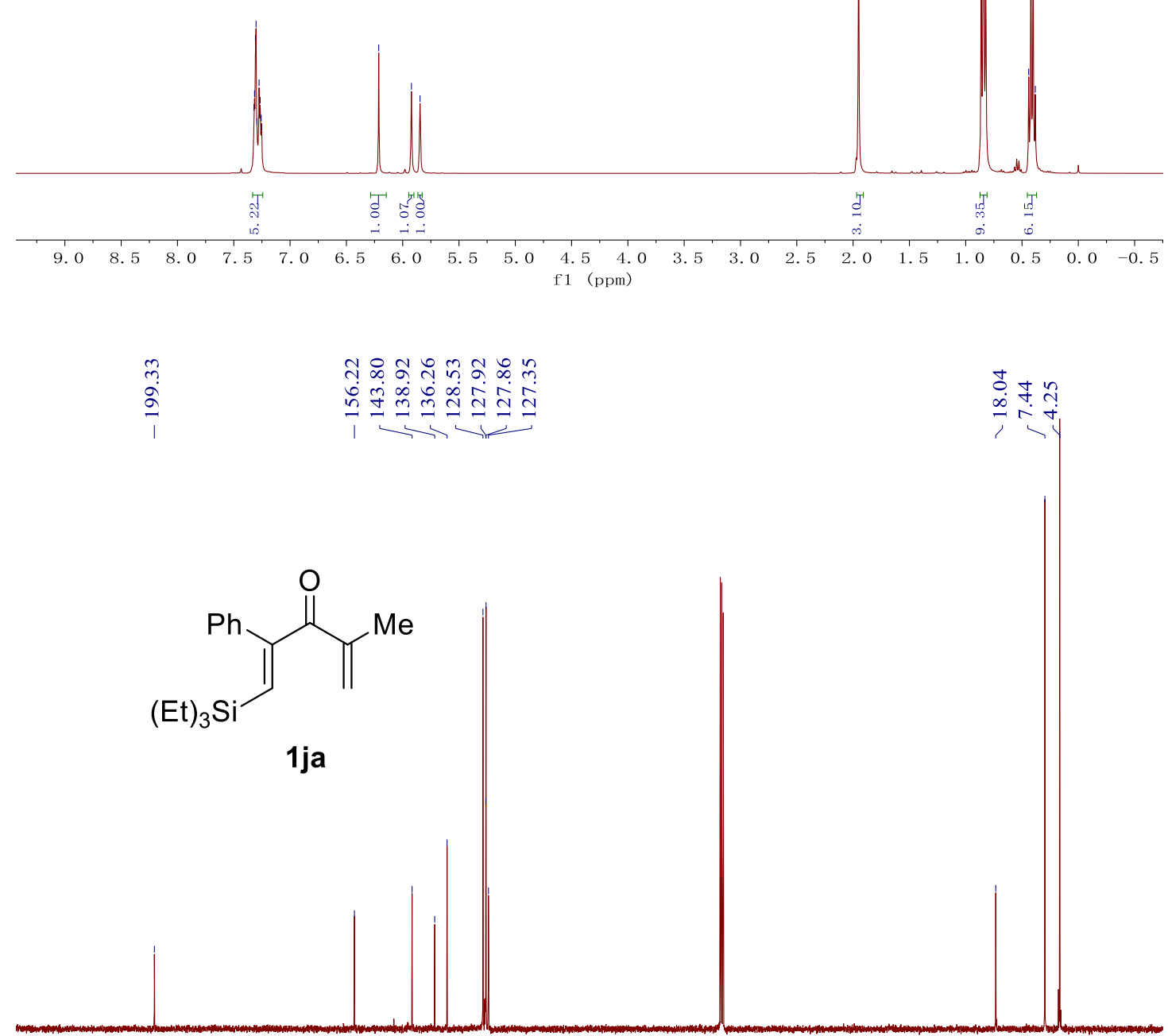

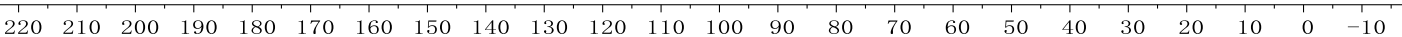
f1 (ppm) 


\section{(Z)-4-methyl-2-phenyl-1-(trimethylsilyl)penta-1,4-dien-3-one (1la)}

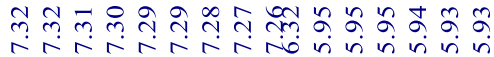

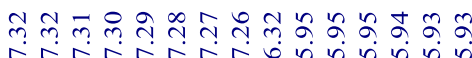

risararon

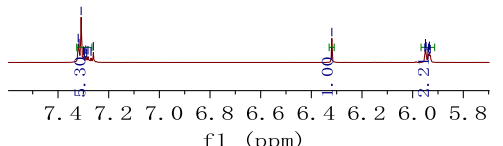<smiles>C=C(C)C(=O)/C(=C\C(C)C)c1ccccc1</smiles>

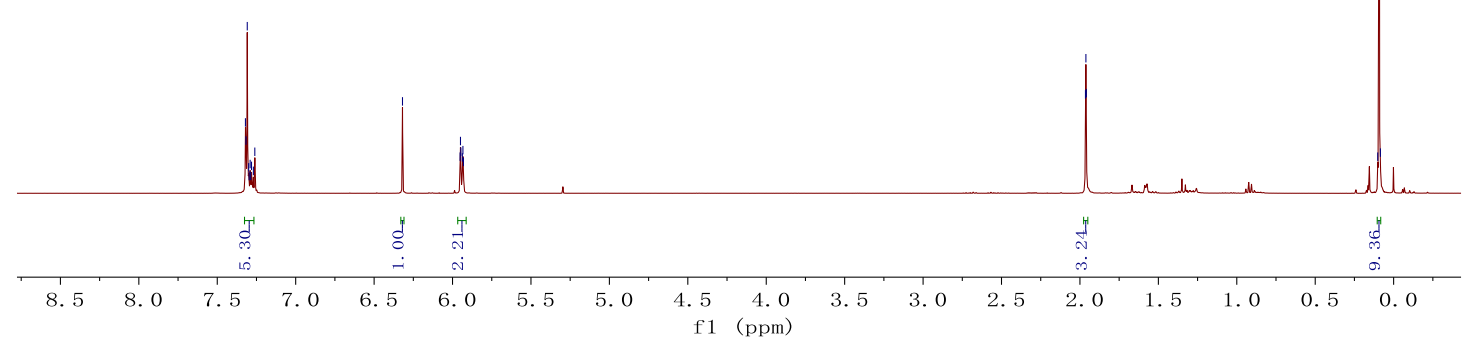

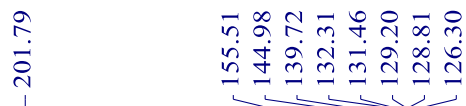

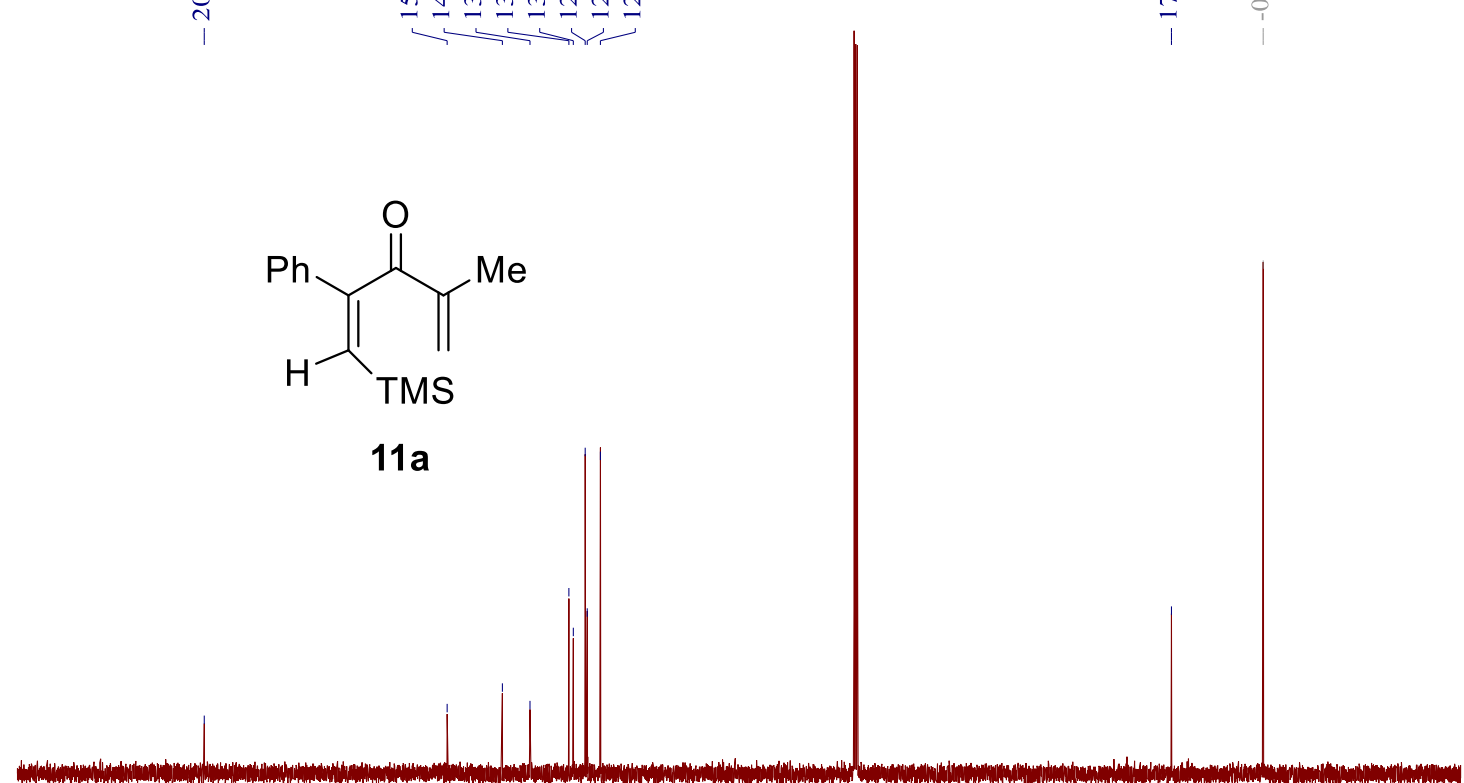

$\begin{array}{lllllllllllllllllllllllllllllllllllllllllll}230 & 220 & 210 & 200 & 190 & 180 & 170 & 160 & 150 & 140 & 130 & 120 & 110 & 100 & 90 & 80 & 70 & 60 & 50 & 40 & 30 & 20 & 10 & 0 & -10 & -20 & -30\end{array}$ f1 (ppm) 


\section{5-methyl-2-phenylcyclopent-2-en-1-one (2aa)}

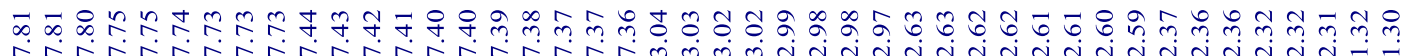
क

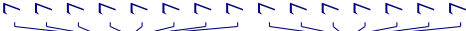
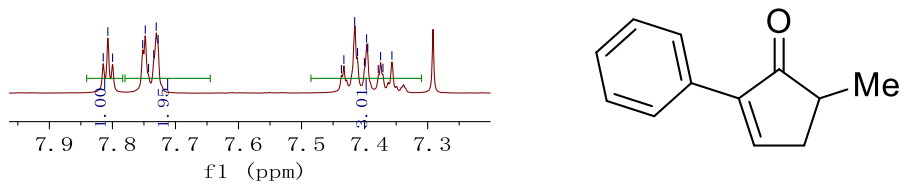

2aa

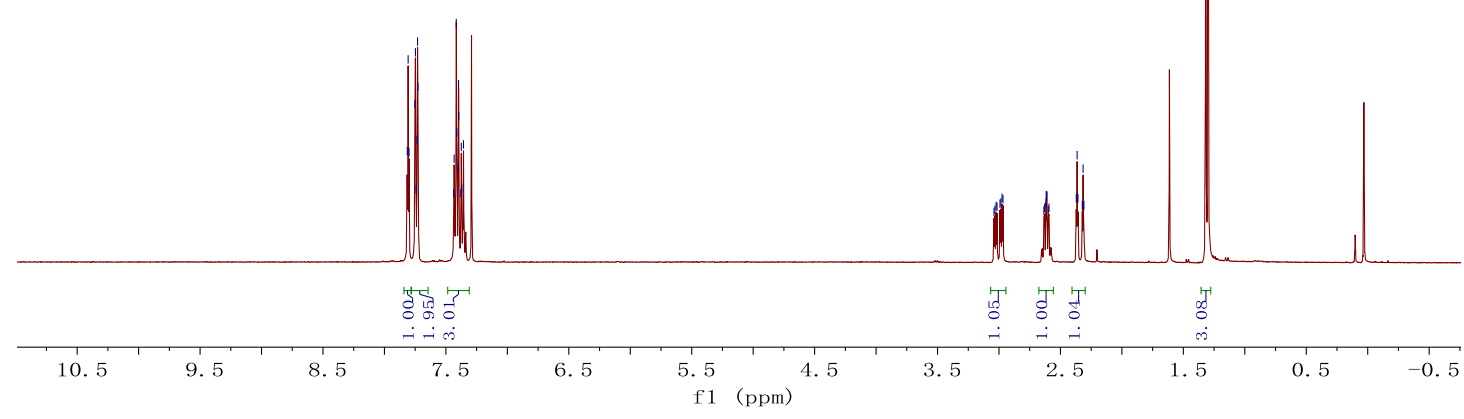<smiles>CC1CC=C(c2ccccc2)C1=O</smiles>

2aa

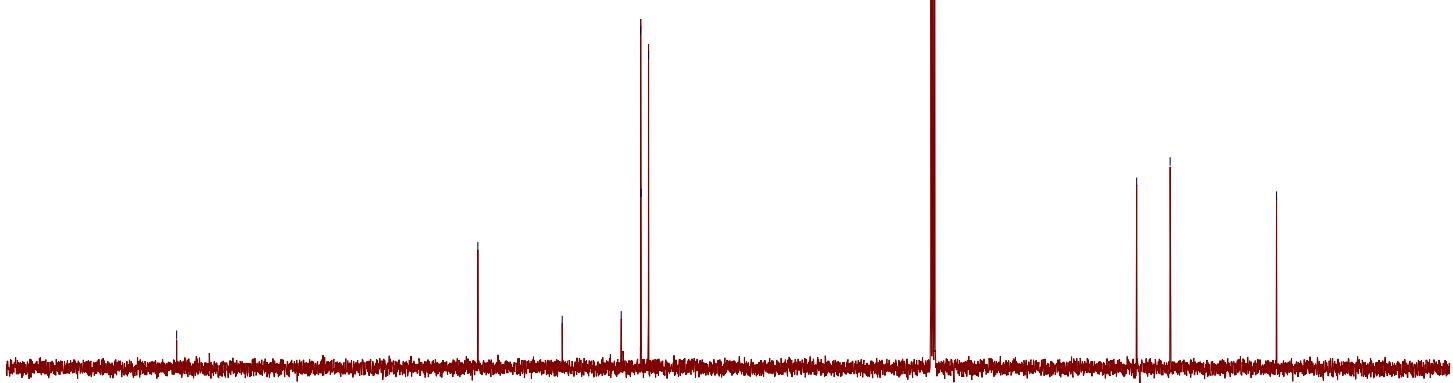

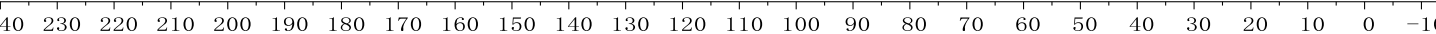
f1 (ppm) 
2-(4-methoxyphenyl)-5-methylcyclopent-2-en-1-one (2ab)
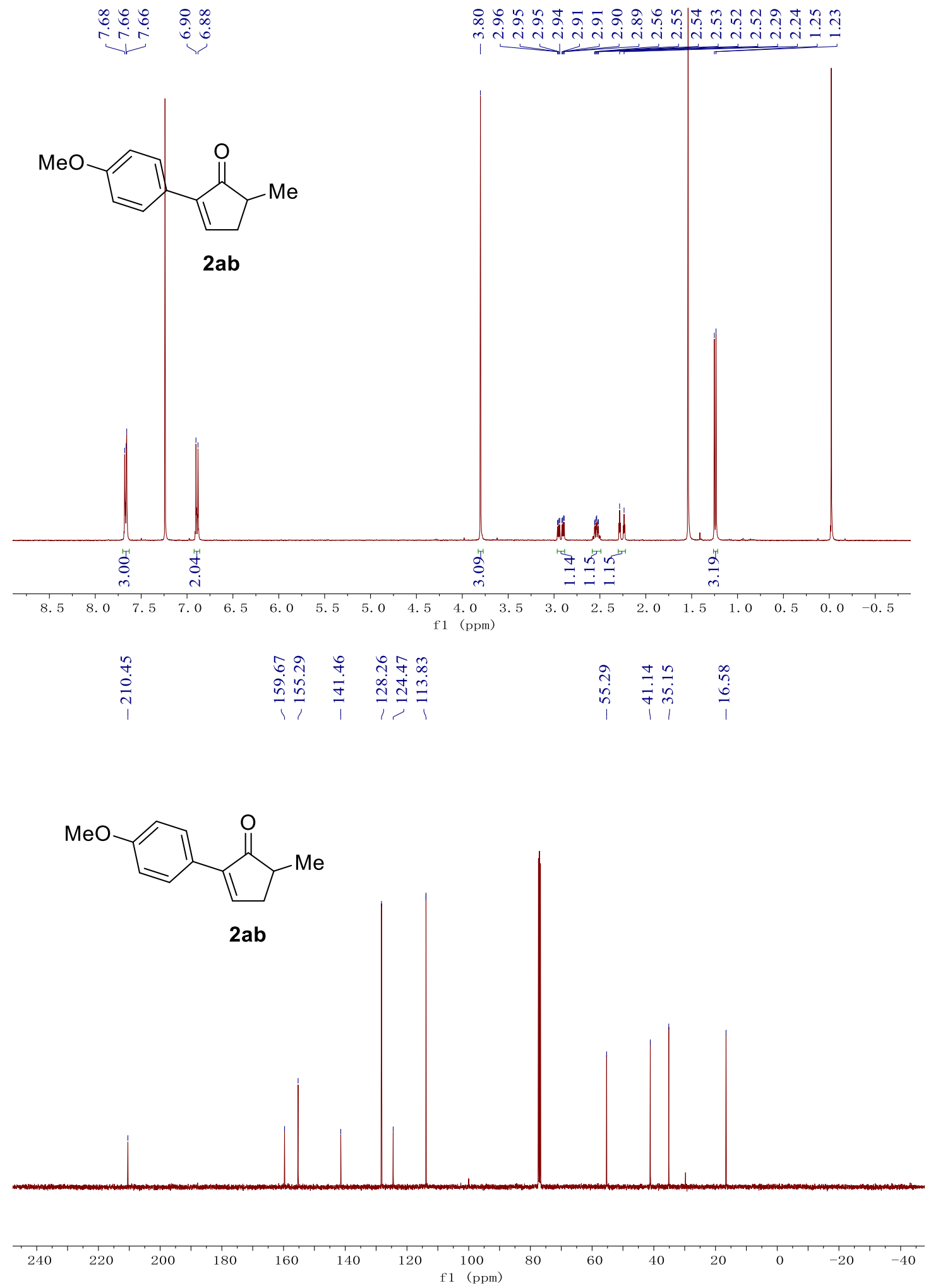


\section{5-methyl-2-(p-tolyl)cyclopent-2-en-1-one (2ac)}

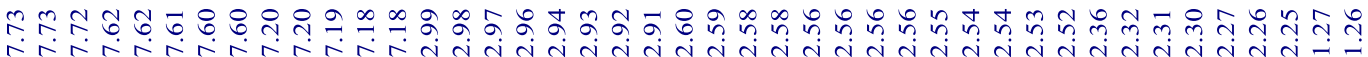

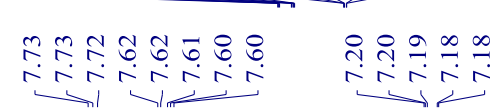

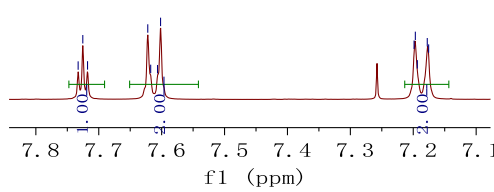<smiles>Cc1ccc(C2=CCC(C)C2=O)cc1</smiles>

2ac
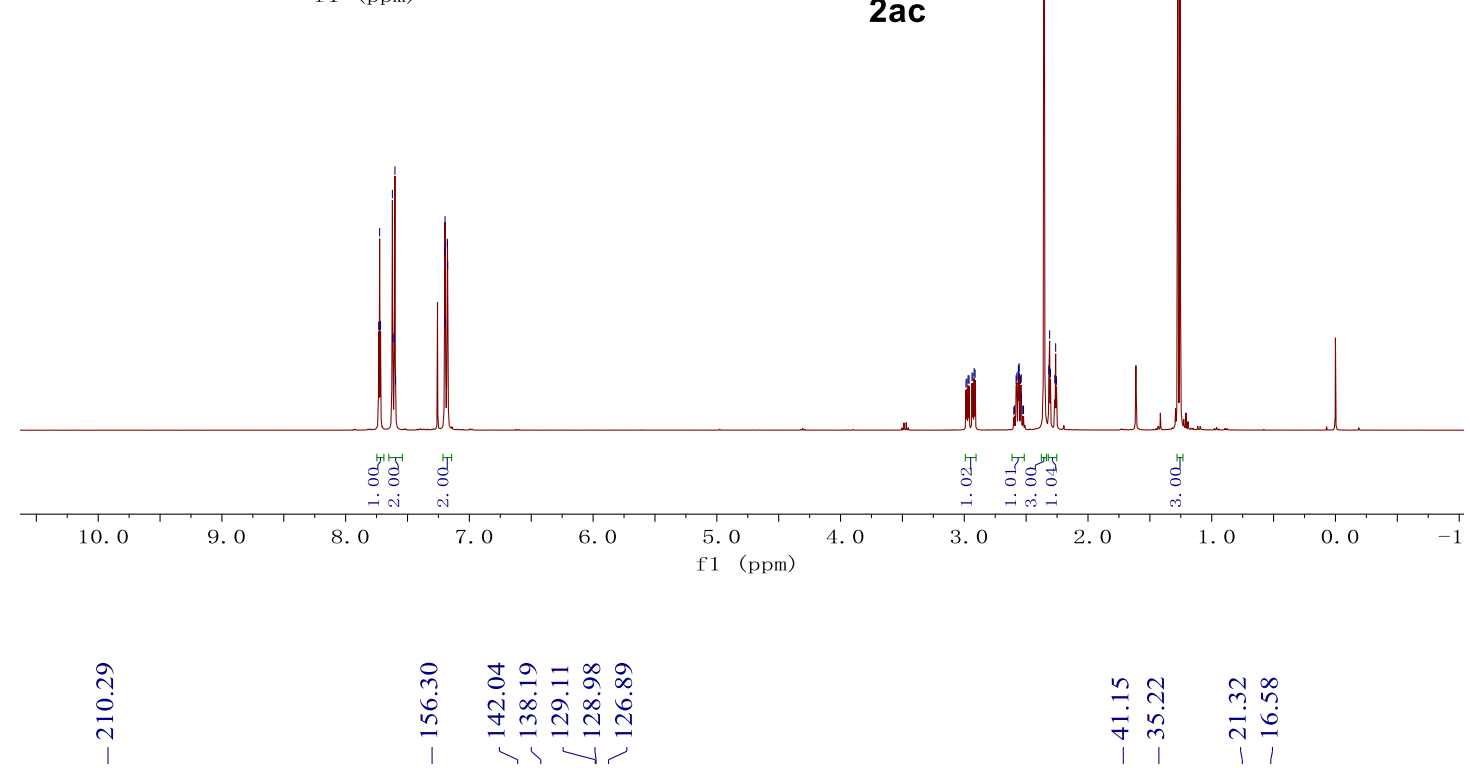<smiles>Cc1ccc(C2=CCC(C)C2=O)cc1</smiles>

2ac 


\section{2-(4-fluorophenyl)-5-methylcyclopent-2-en-1-one (2ad)}

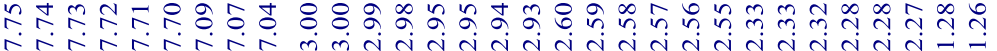

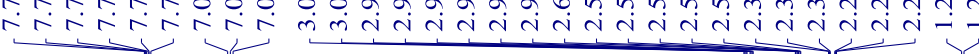

そุ

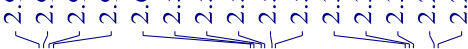
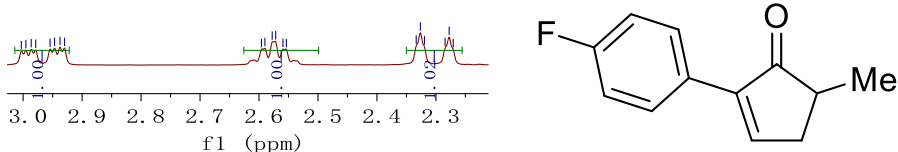

2ad
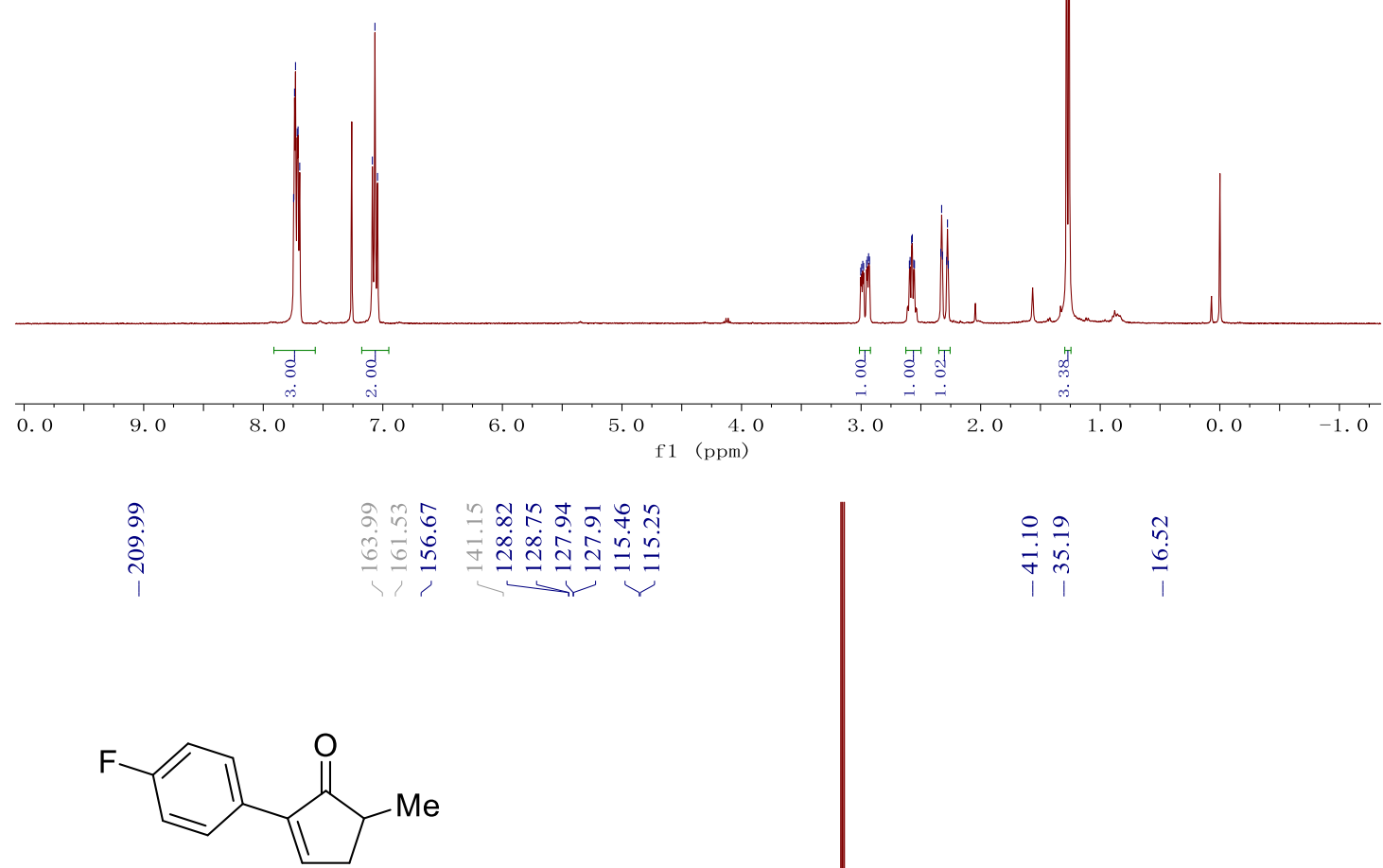

2ad 


\section{2-(4-chlorophenyl)-5-methylcyclopent-2-en-1-one (2ae)}

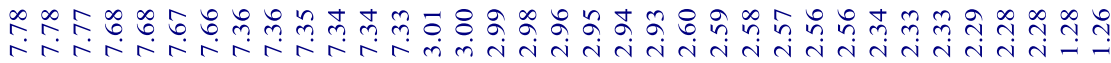<smiles>CC1CC=C(c2ccc(Cl)cc2)C1=O</smiles>

2ae

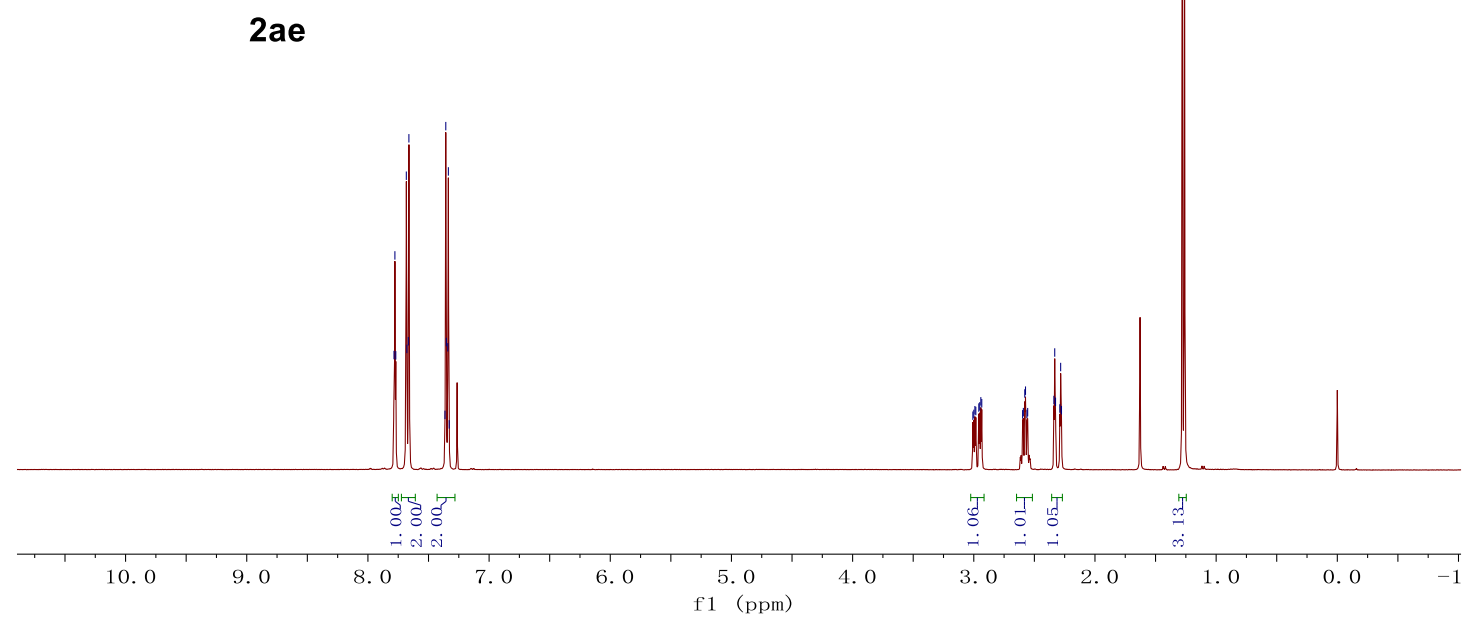<smiles>CC1CC=C(c2ccc(Cl)cc2)C1=O</smiles>

2ae 


\section{2-(4-bromophenyl)-5-methylcyclopent-2-en-1-one (2af)}

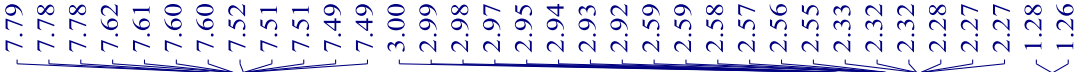

\section{शे⿻日禸}

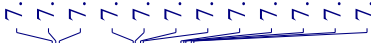
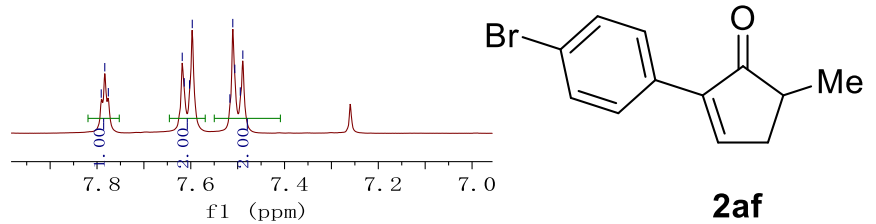

2af

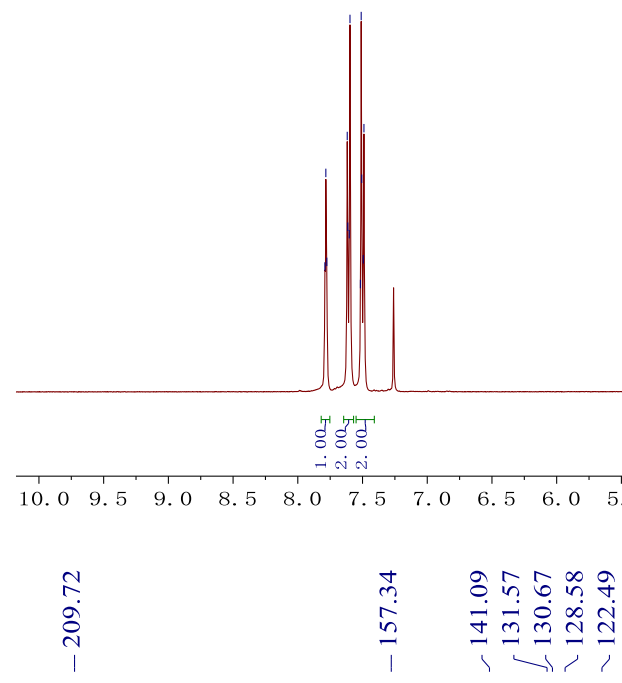<smiles>CC1CC=C(c2ccc(Br)cc2)C1=O</smiles>

2af

$\begin{array}{lllllllllllll}210 & 200 & 190 & 180 & 170 & 160 & 150 & 140 & 130 & 120 & 110 & 100 & 90\end{array}$

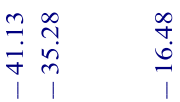


5-methyl-2-(4-(trifluoromethyl)phenyl)cyclopent-2-en-1-one (2ag)

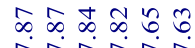

Ninj

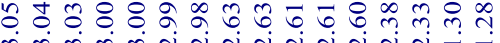

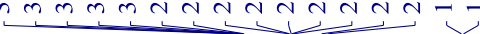

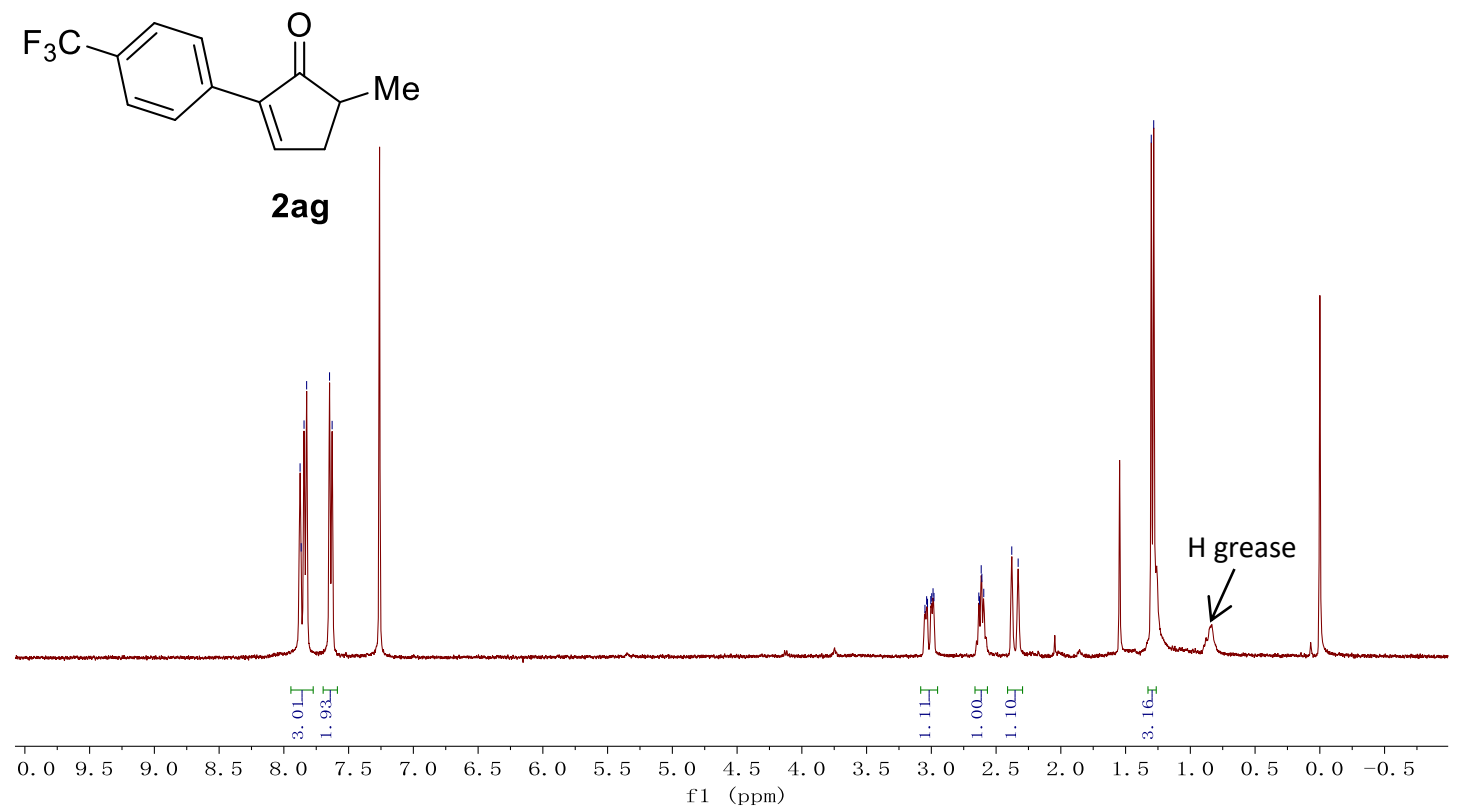

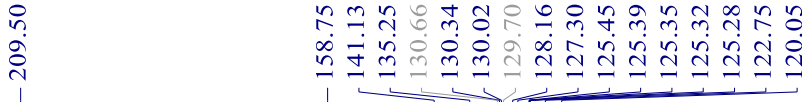

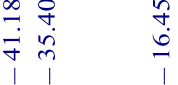

$\mathrm{F}_{3} \mathrm{C}$

2ag

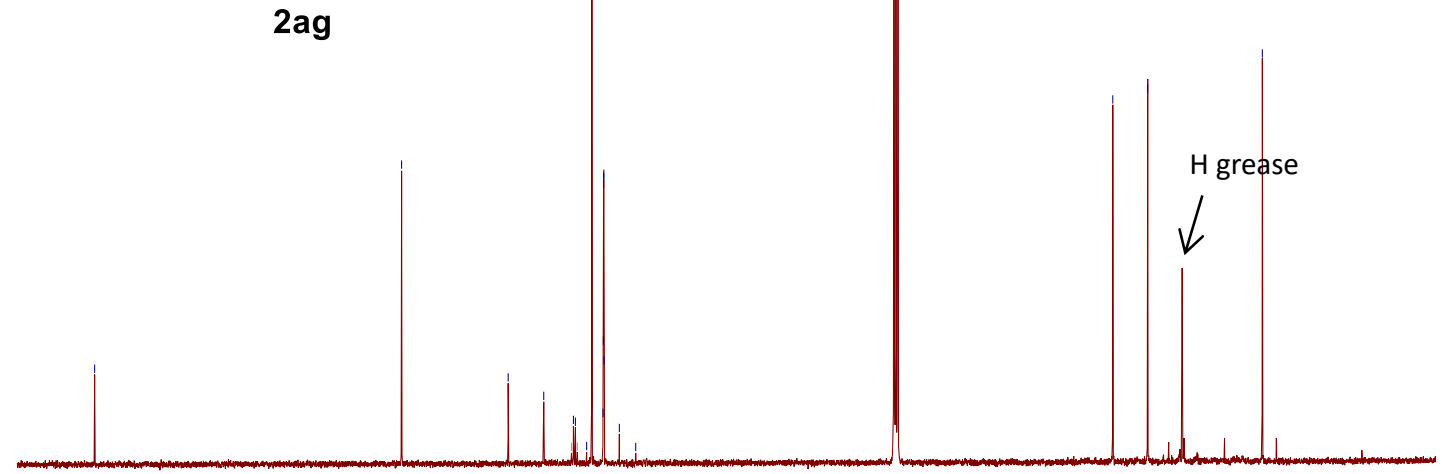

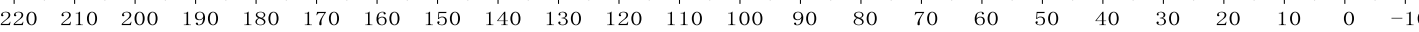




\section{5-methyl-2-(m-tolyl)cyclopent-2-en-1-one (2ah)}

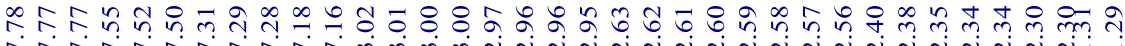

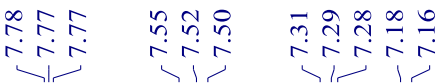
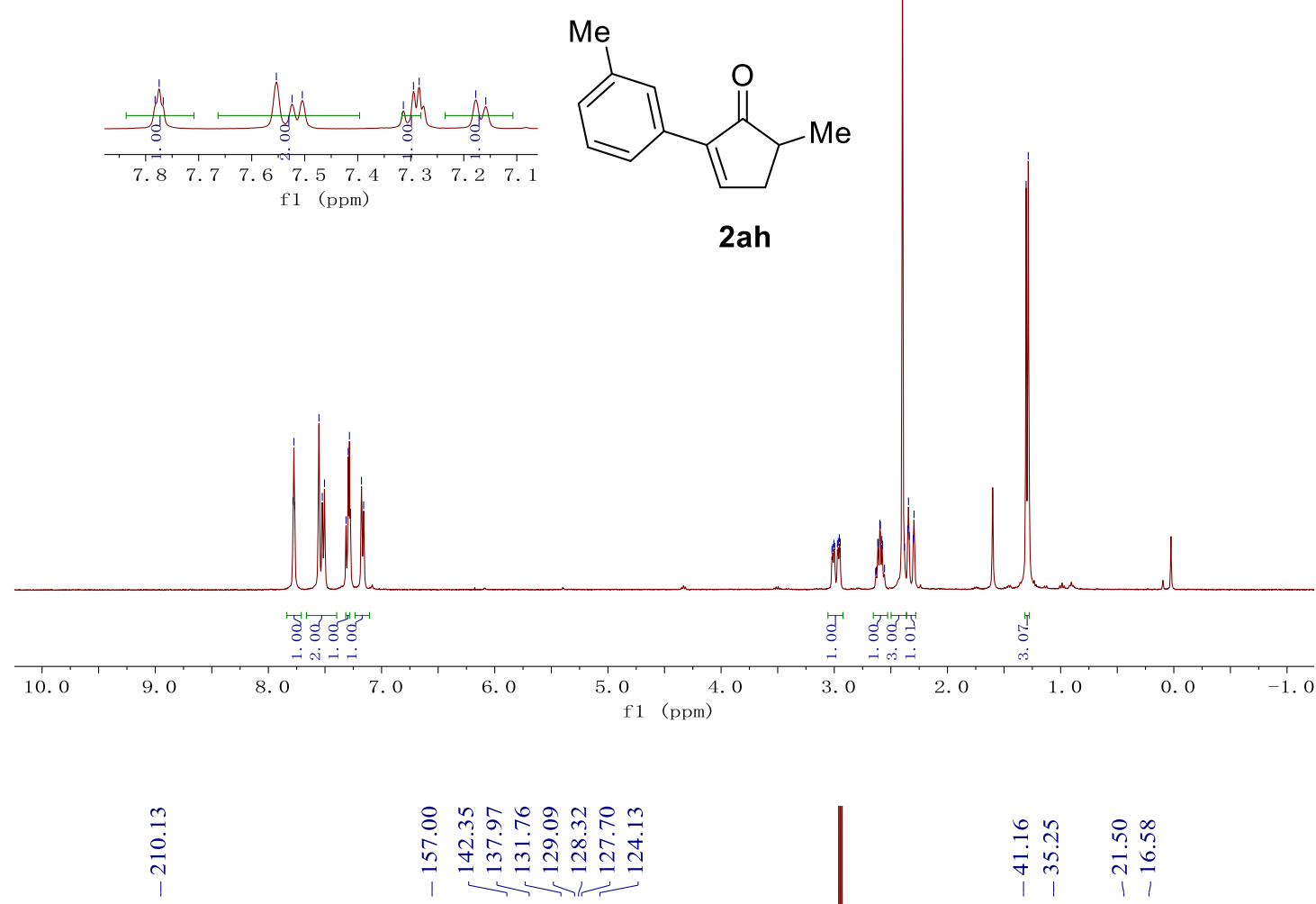

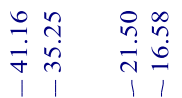<smiles>Cc1cccc(C2=CCC(C)C2=O)c1</smiles>

2ah

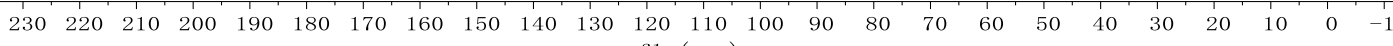
f1 (ppm) 


\section{5-methyl-2-(o-tolyl)cyclopent-2-en-1-one (2ai)}

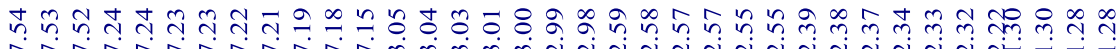

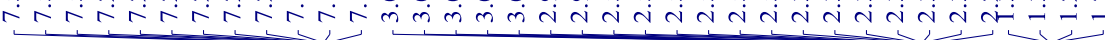

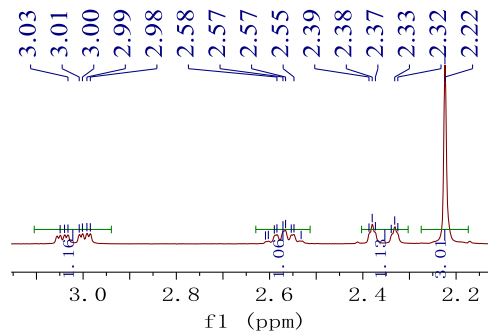<smiles>Cc1ccccc1C1=CCC(C)C1=O</smiles>

2ai<smiles>Cc1ccccc1C1=CCC(C)C1=O</smiles>

2ai

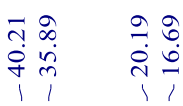

$\begin{array}{llllllllllllllllllllllllll}220 & 210 & 200 & 190 & 180 & 170 & 160 & 150 & 140 & 130 & 120 & 110 & 100 & 90 & 80 & 70 & 60 & 50 & 40 & 30 & 20 & 10 & (\end{array}$ f1 (ppm) 
2-(benzo[d][1,3]dioxol-5-yl)-5-methylcyclopent-2-en-1-one (2aj)

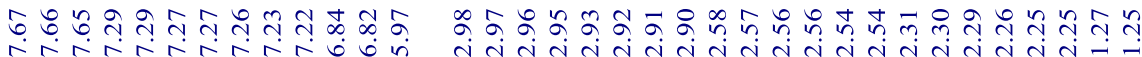

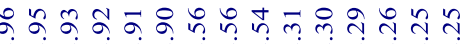

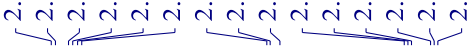

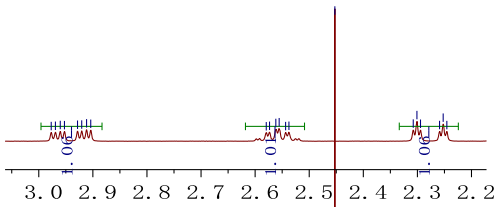<smiles>[C]C1CC=C(c2ccc3c(c2)OCO3)C1=O</smiles>
f1 (ppm)

2aj

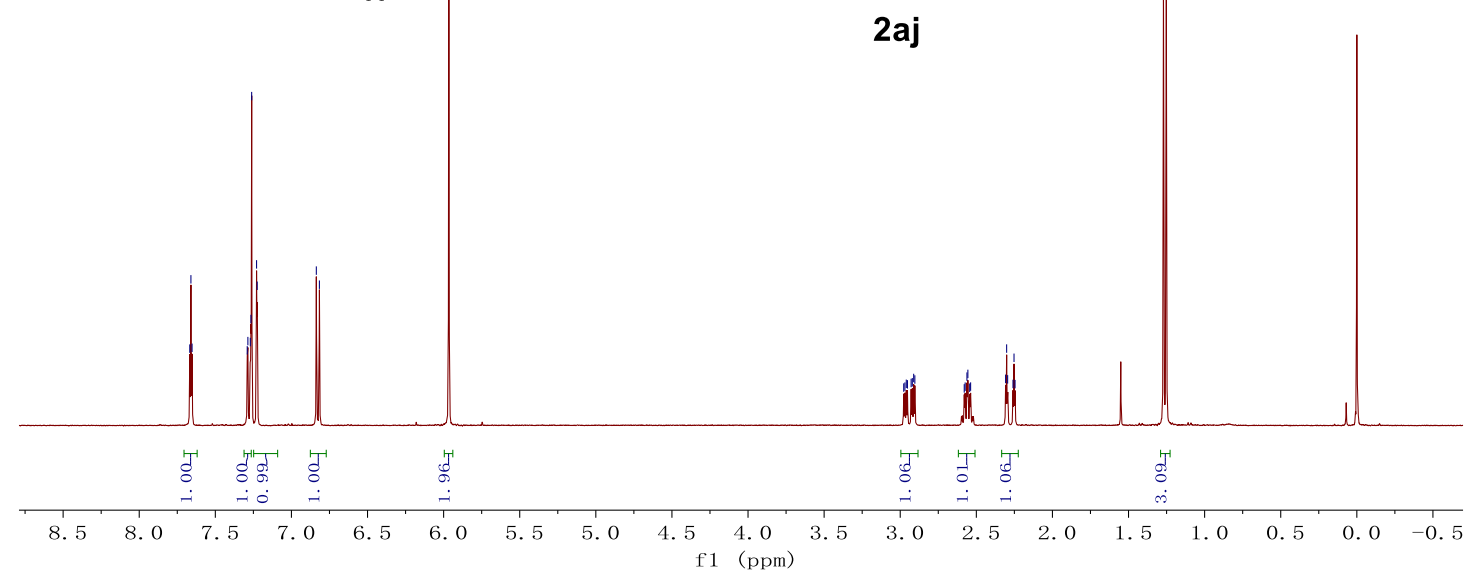

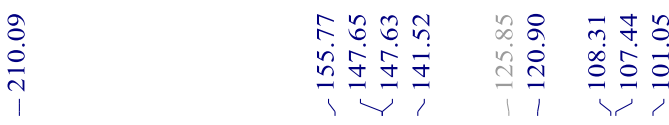

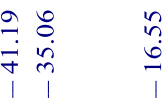<smiles>CC1CC=C(c2ccc3c(c2)OCO3)C1=O</smiles>

2aj

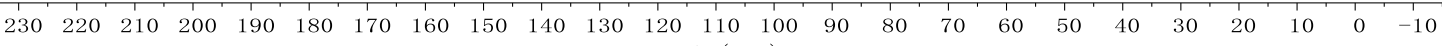
f1 (ppm) 


\section{5-methyl-2-(naphthalen-2-yl)cyclopent-2-en-1-one (2ak)}

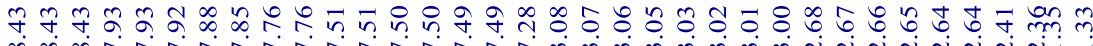

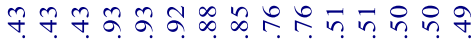

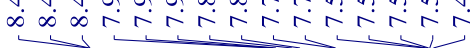
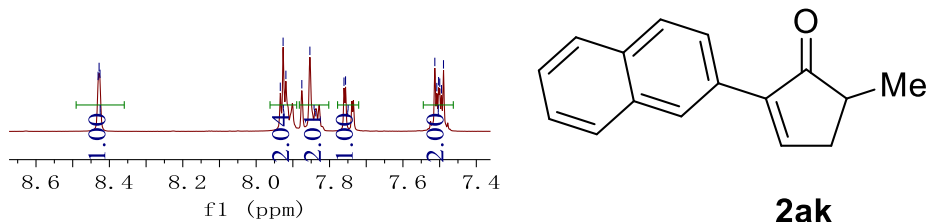

2ak
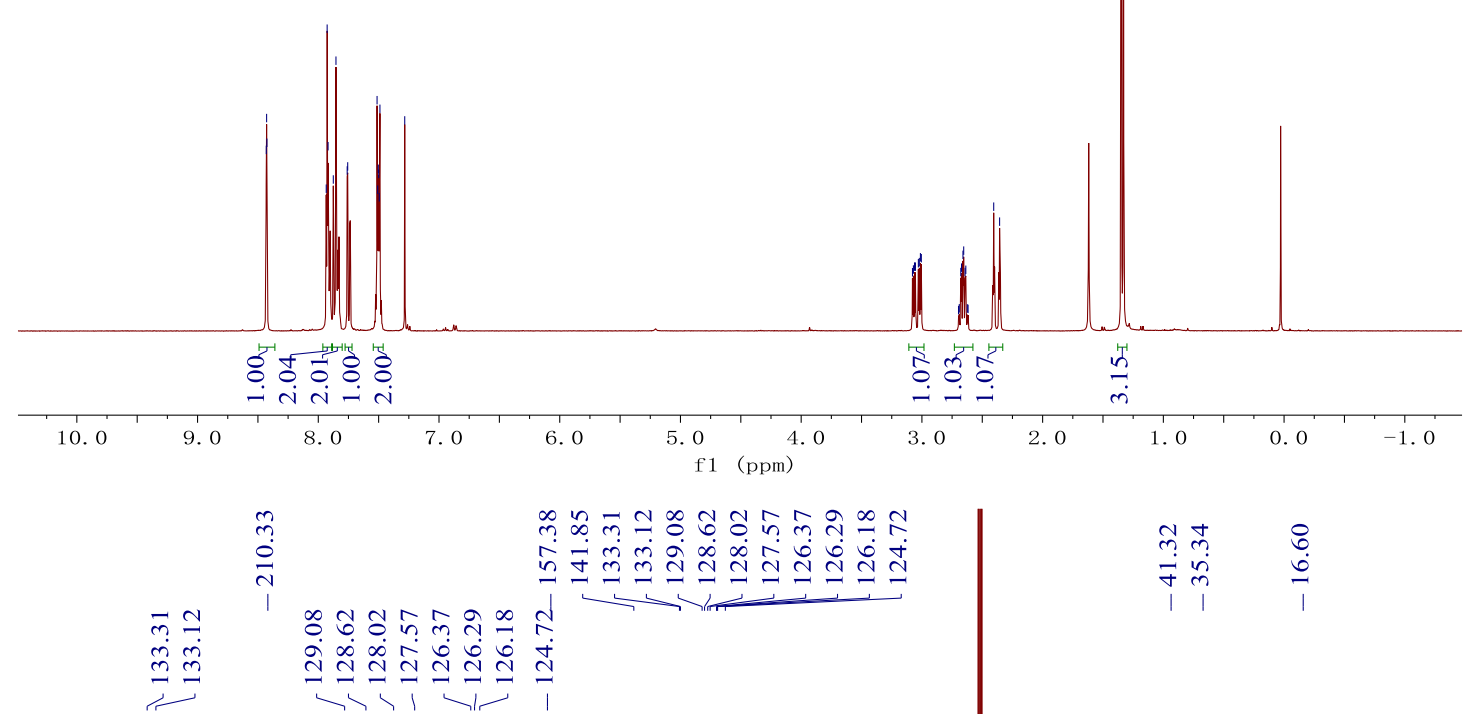

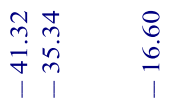

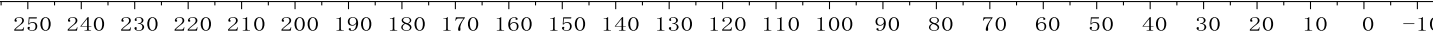
f1 (ppm) 


\section{5-methyl-2-(thiophen-2-yl)cyclopent-2-en-1-one (2al)}

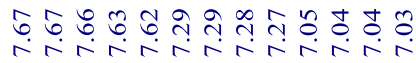

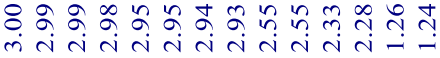

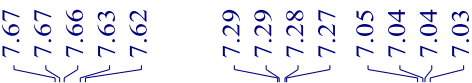
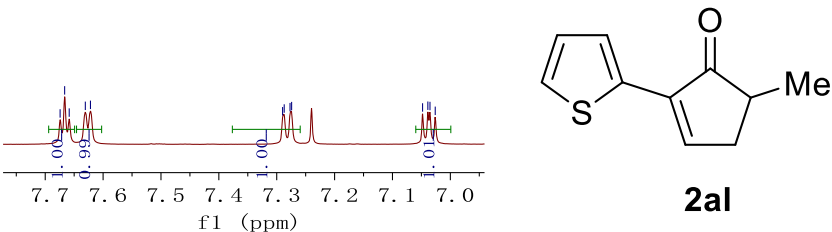

2al
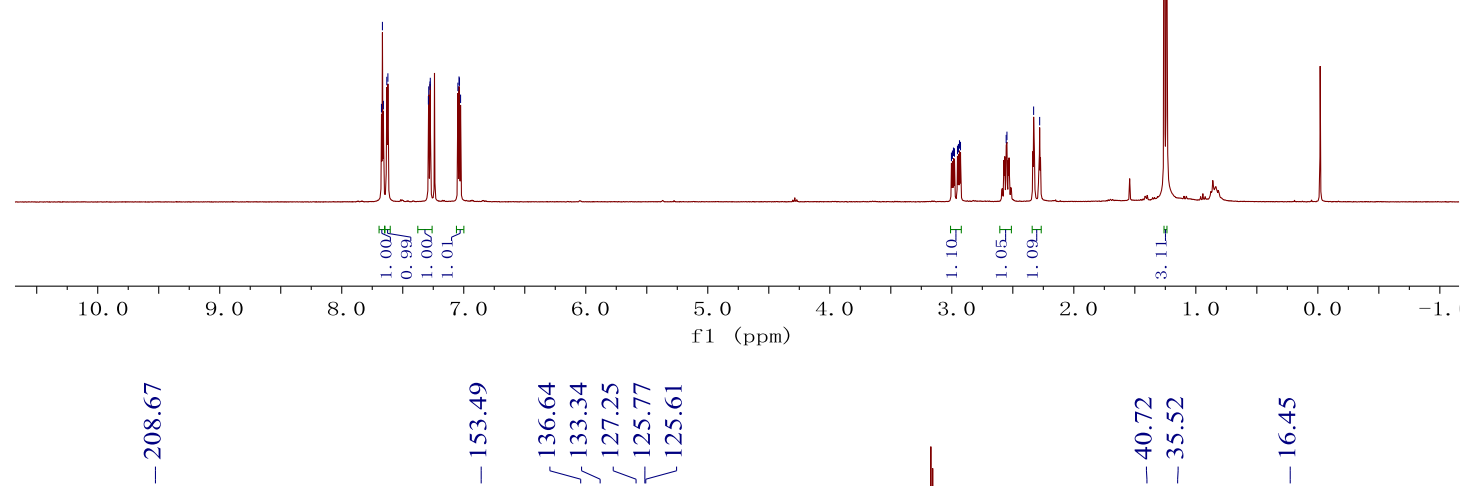

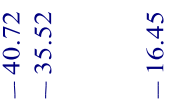<smiles>CC1CC=C(c2cccs2)C1=O</smiles>

2al

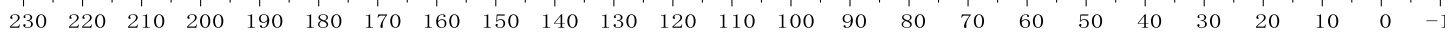
f1 (ppm) 


\section{5-ethyl-2-phenylcyclopent-2-en-1-one (2ba)}

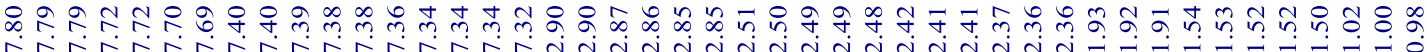
œ

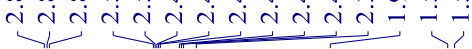

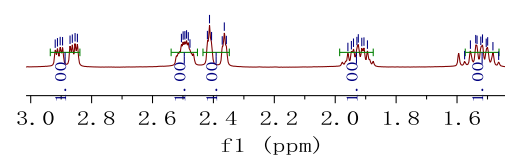

f1 (ppm)

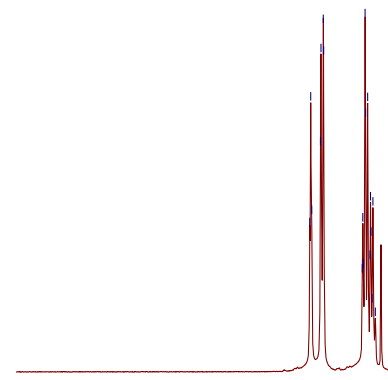

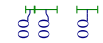<smiles>CCC1CC=C(c2ccccc2)C1=O</smiles>

$2 b a$

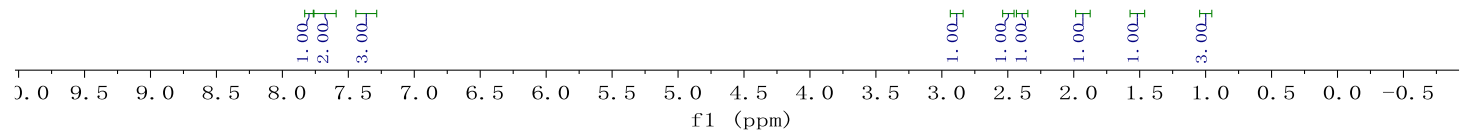<smiles>CCC1CC=C(c2ccccc2)C1=O</smiles>

2ba

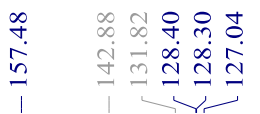

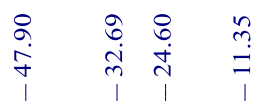

$\begin{array}{llllllllllllllllllllllllllllllllll}230 & 220 & 210 & 200 & 190 & 180 & 170 & 160 & 150 & 140 & 130 & 120 & 110 & 100 & 90 & 80 & 70 & 60 & 50 & 40 & 30 & 20 & 10 & 0\end{array}$ f1 (ppm) 
2-phenyl-5-propylcyclopent-2-en-1-one (2bb)

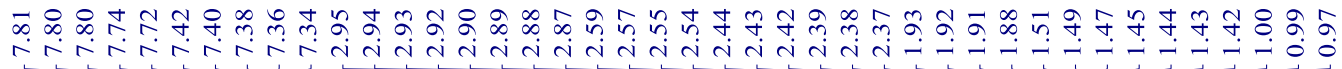

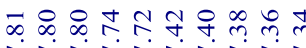

inisivinis

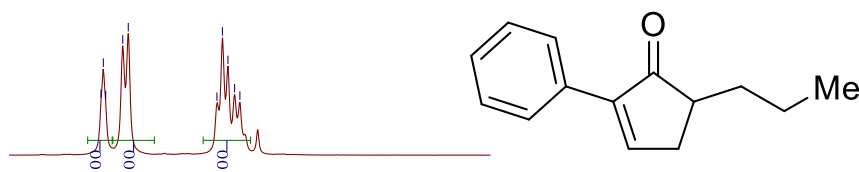

$\begin{array}{llllllllllllll}8.0 & 7.8 & 7.6 & 7.4 & 7.2 & 7.0 & 6.8 & 6.6 & \mathbf{2 b}\end{array}$

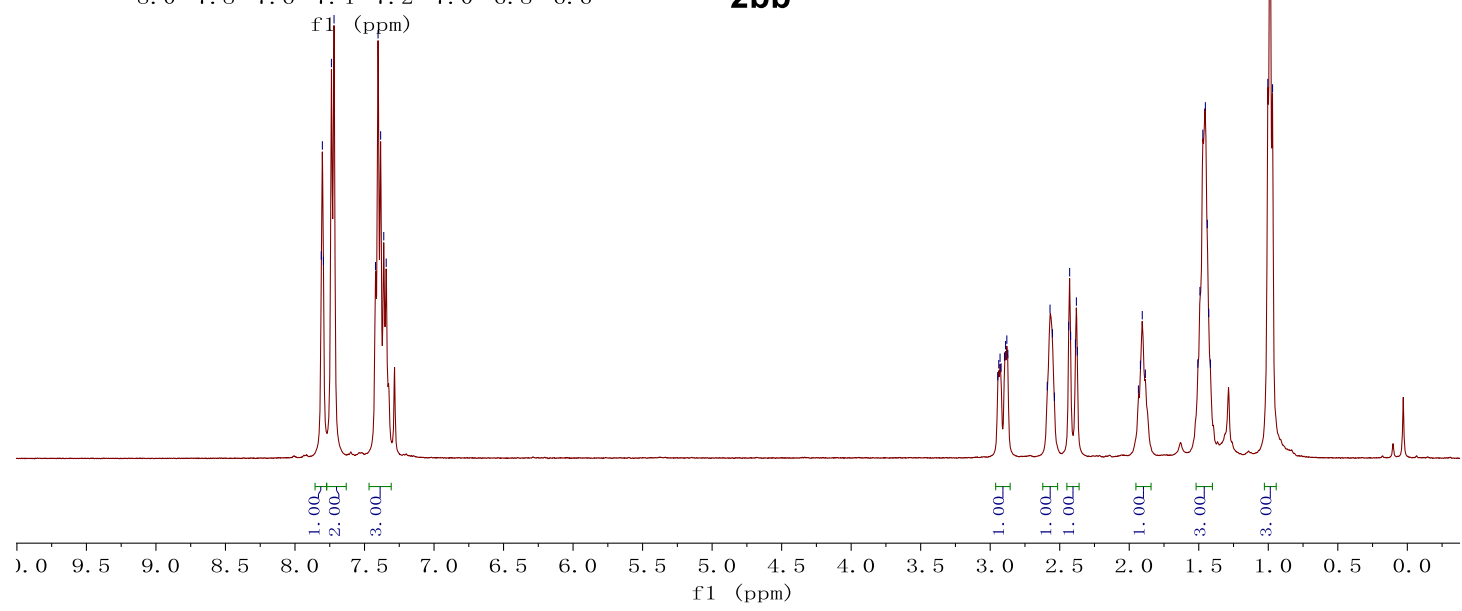

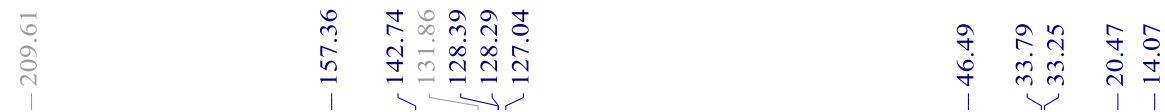

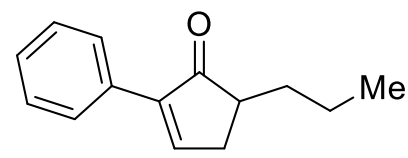

$2 b b$

$\begin{array}{lllllllllllllllllllllllllll}230 & 220 & 210 & 200 & 190 & 180 & 170 & 160 & 150 & 140 & 130 & 120 & 110 & 100 & 90 & 80 & 70 & 60 & 50 & 40 & 30 & 20 & 10 & 0 & -1\end{array}$ f1 (ppm) 


\section{5-hexyl-2-phenylcyclopent-2-en-1-one (2bc)}

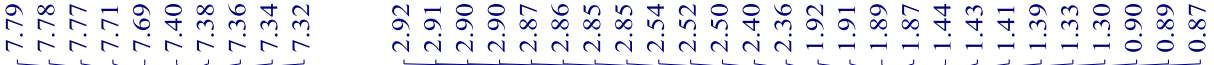

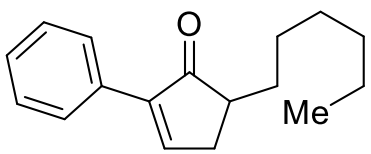

2bc
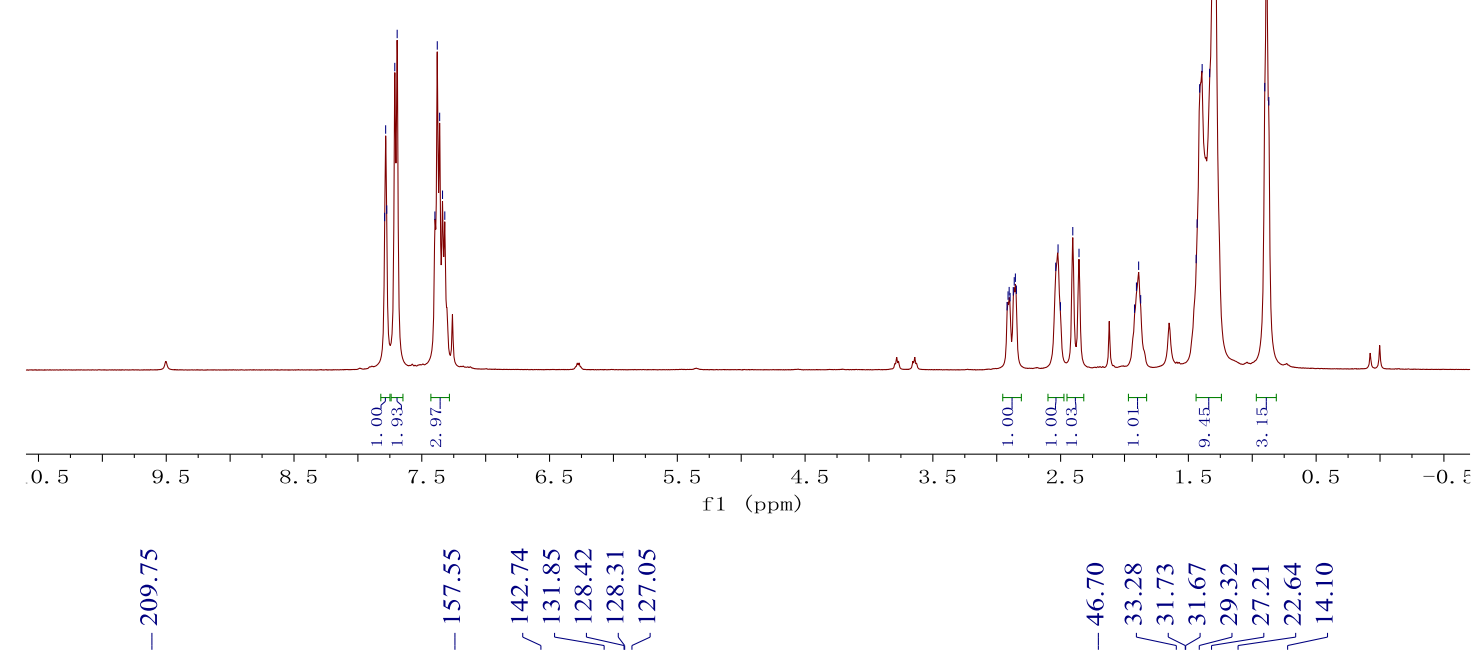

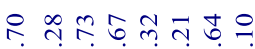

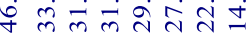<smiles>CCCCCCC1CC=C(c2ccccc2)C1=O</smiles>

$2 b c$

$\begin{array}{llllllllllllllllllllllllllllll}230 & 220 & 210 & 200 & 190 & 180 & 170 & 160 & 150 & 140 & 130 & 120 & 110 & 100 & 90 & 80 & 70 & 60 & 50 & 40 & 30 & 20 & 10 & 0 & -10\end{array}$ f1 ( $\mathrm{ppm})$ 
5-isopropyl-2-phenylcyclopent-2-en-1-one (2bd)

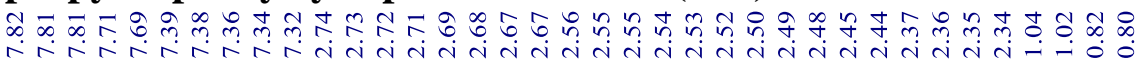

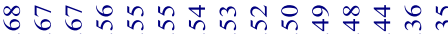

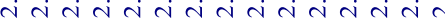
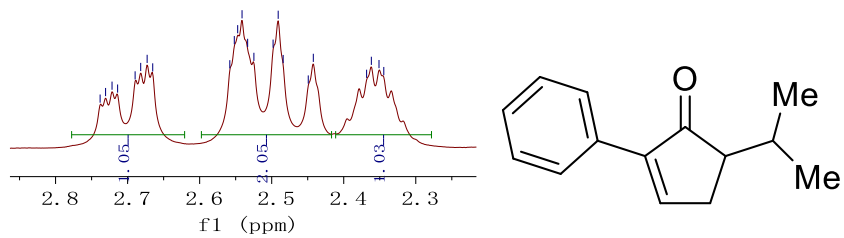

$2 \mathbf{b d}$
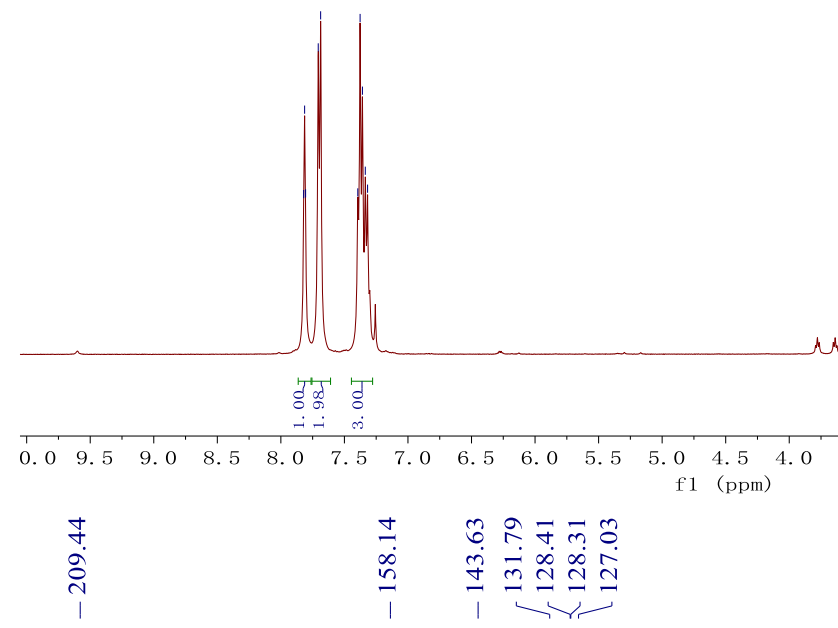

声琶
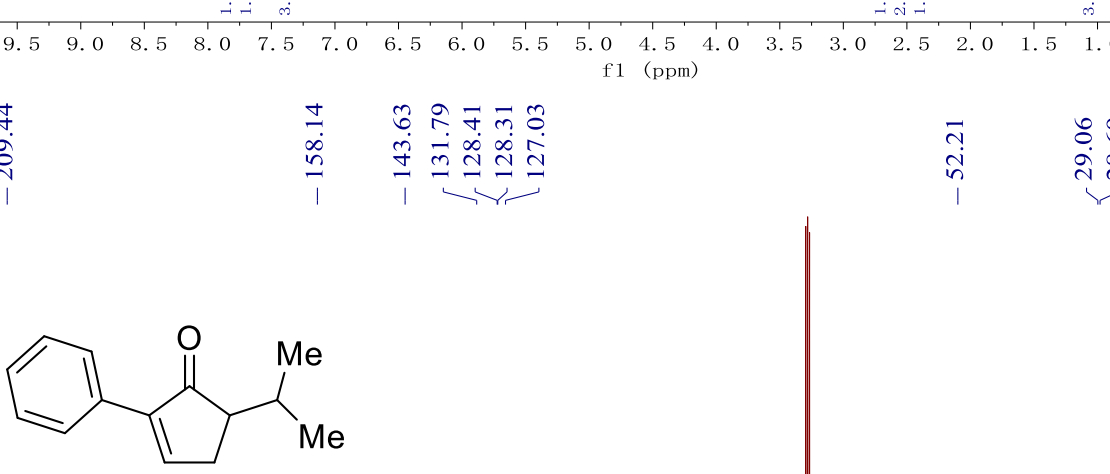

$\mathbf{2 b d}$

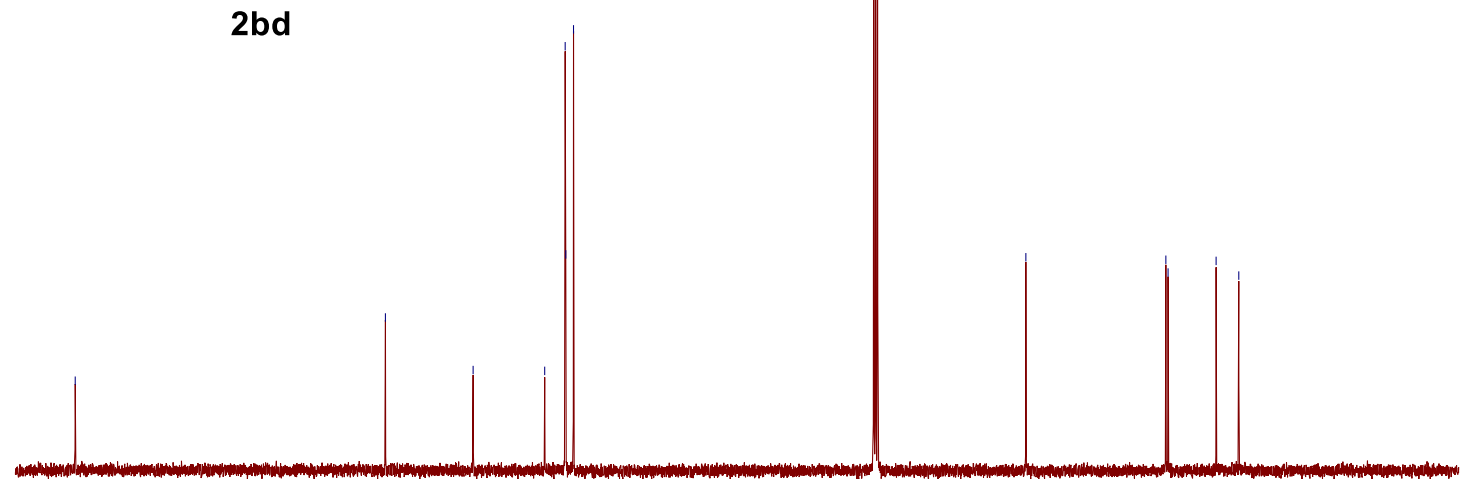

$\begin{array}{lllllllllllllllllllllll}210 & 200 & 190 & 180 & 170 & 160 & 150 & 140 & 130 & 120 & 110 & 100 & 90 & 80 & 70 & 60 & 50 & 40 & 30 & 20 & 10 & 0 & -10\end{array}$ f1 (ppm) 


\section{5-cyclopropyl-2-phenylcyclopent-2-en-1-one (2be)}

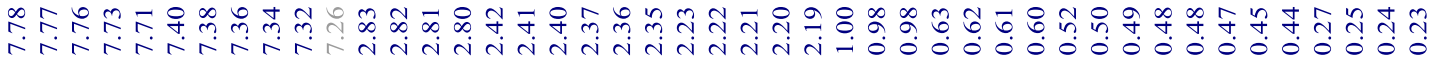

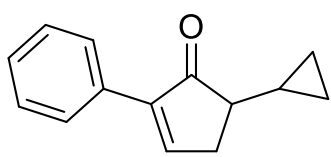

2 be

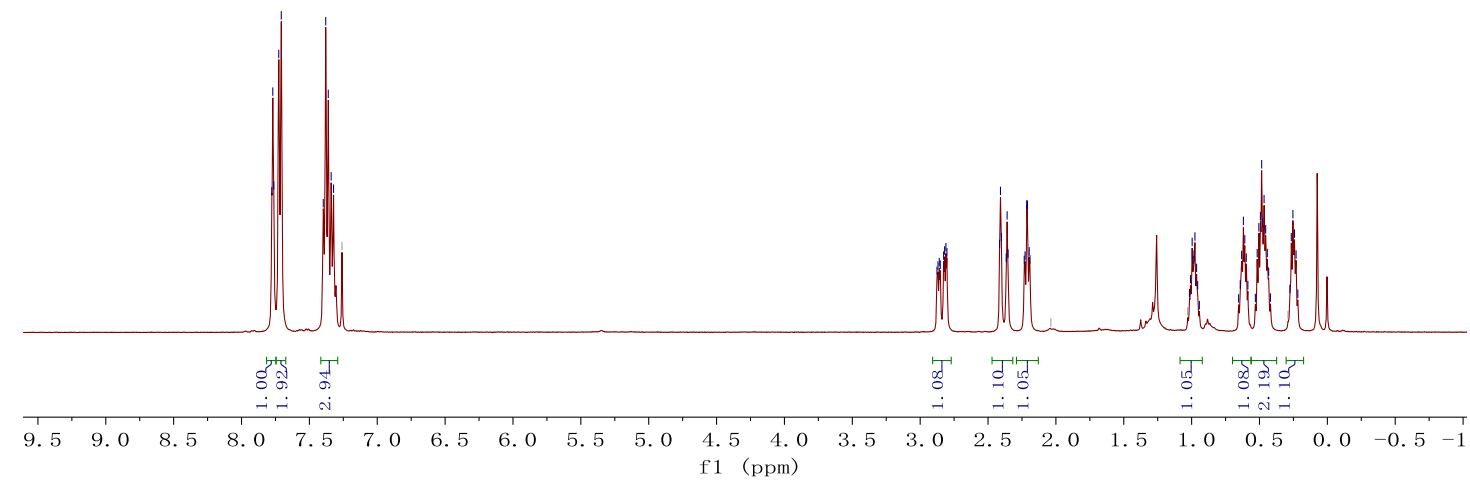<smiles>O=C1C(c2ccccc2)=CCC1C1CC1</smiles>

$2 \mathrm{be}$

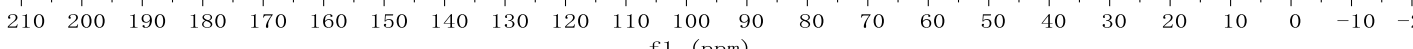
f1 (ppm) 
<smiles>C=CCCC1CC=C(c2ccccc2)C1=O</smiles>

$2 b f$
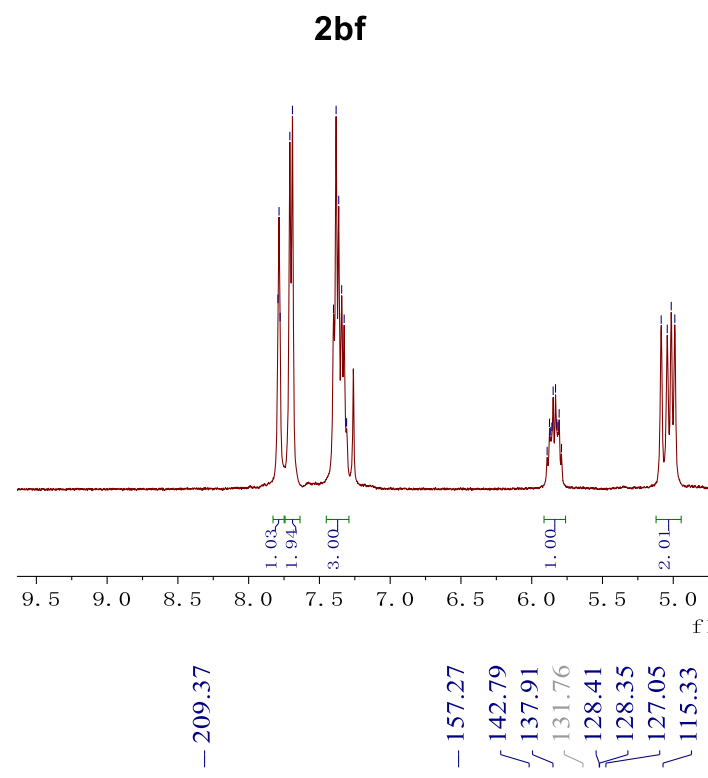

f1 1 (ppm)

管

Im

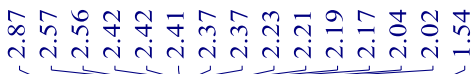

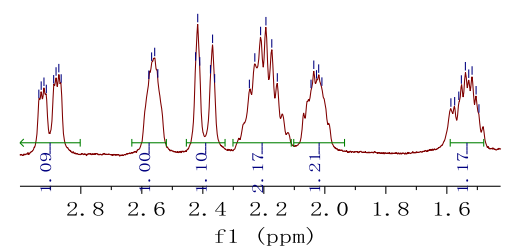

f1 (ppm)<smiles>C=CCCC1CC=C(c2ccccc2)C1=O</smiles>

$2 b f$

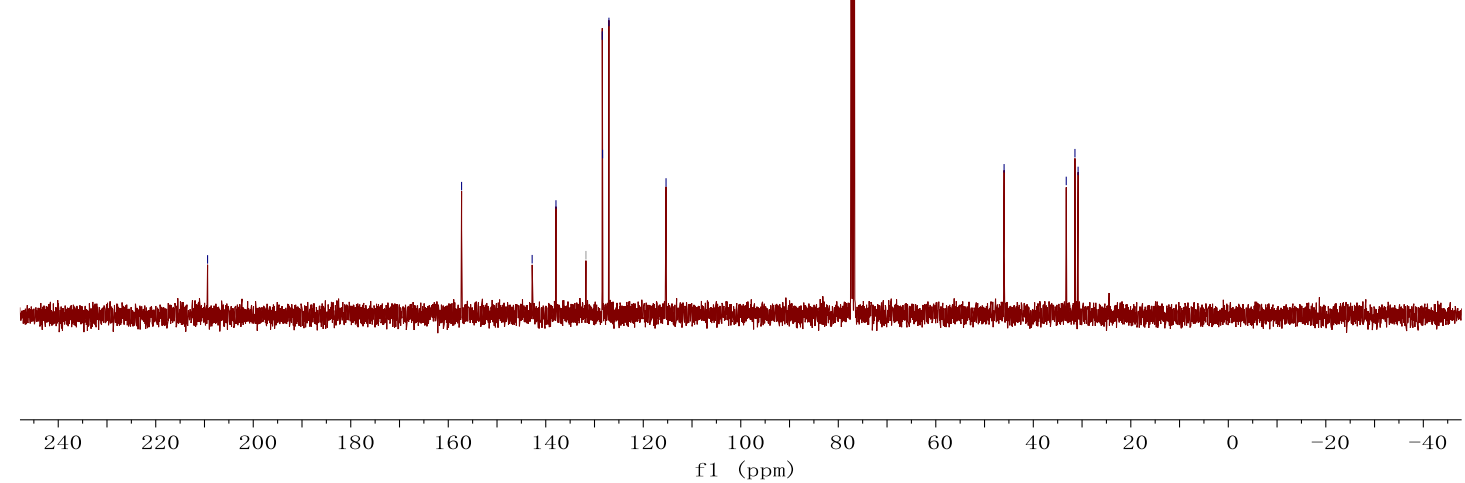




\section{5-(2-chloroethyl)-2-phenylcyclopent-2-en-1-one (2bg)}

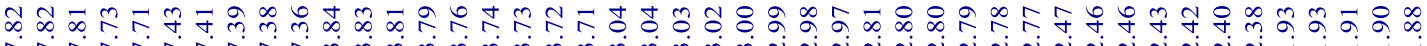

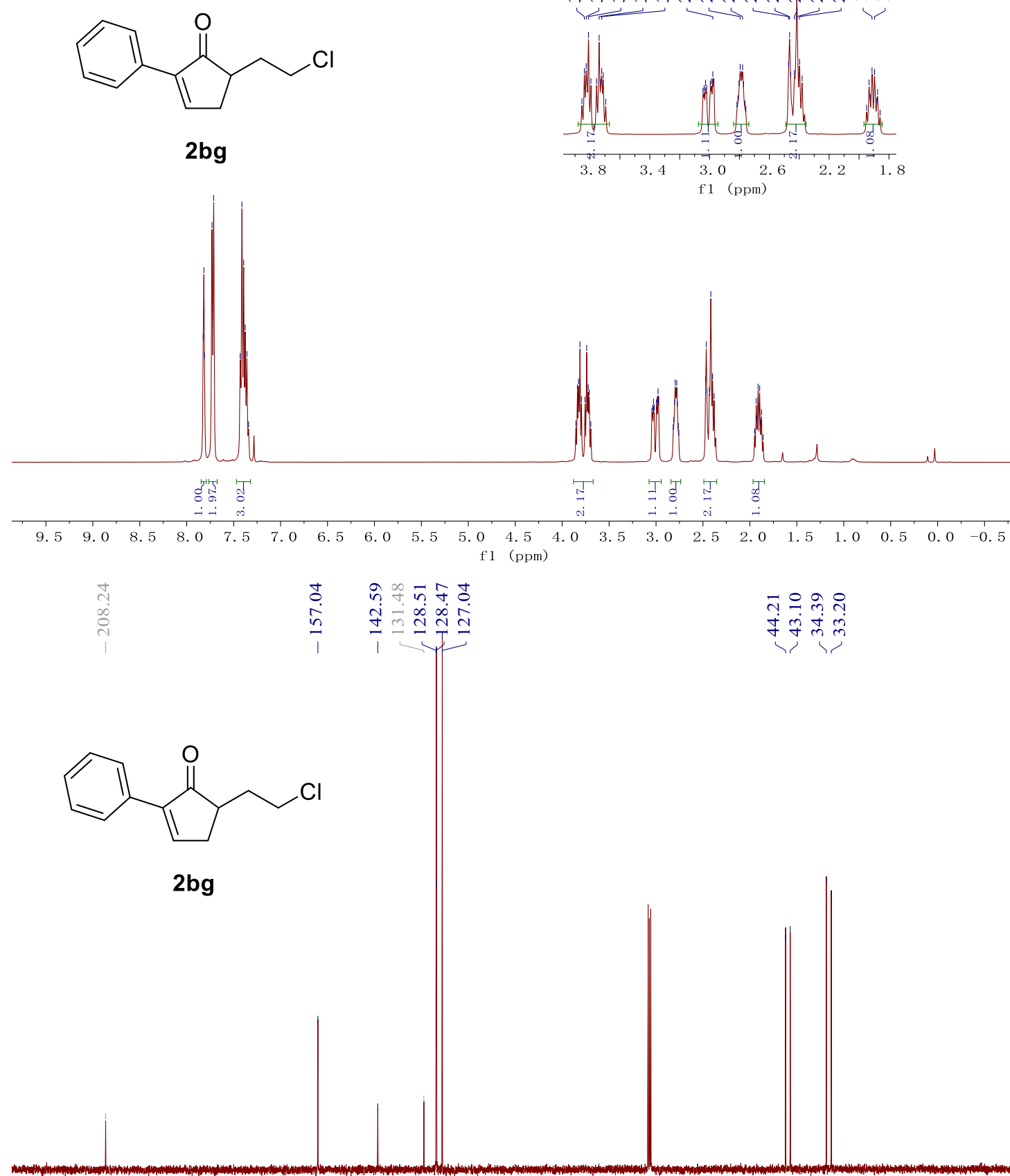

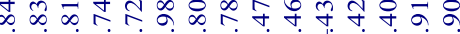

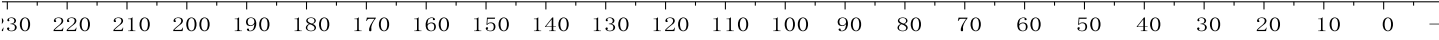
f1 (ppm) 


\section{5-(2-methoxyethyl)-2-phenylcyclopent-2-en-1-one (2bh)}

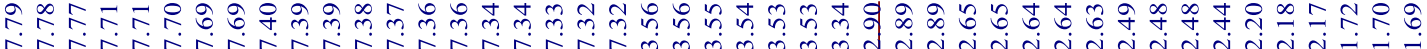

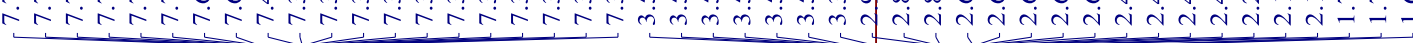<smiles>COCCC1CC=C(c2ccccc2)C1=O</smiles>

\section{$2 \mathrm{bh}$}
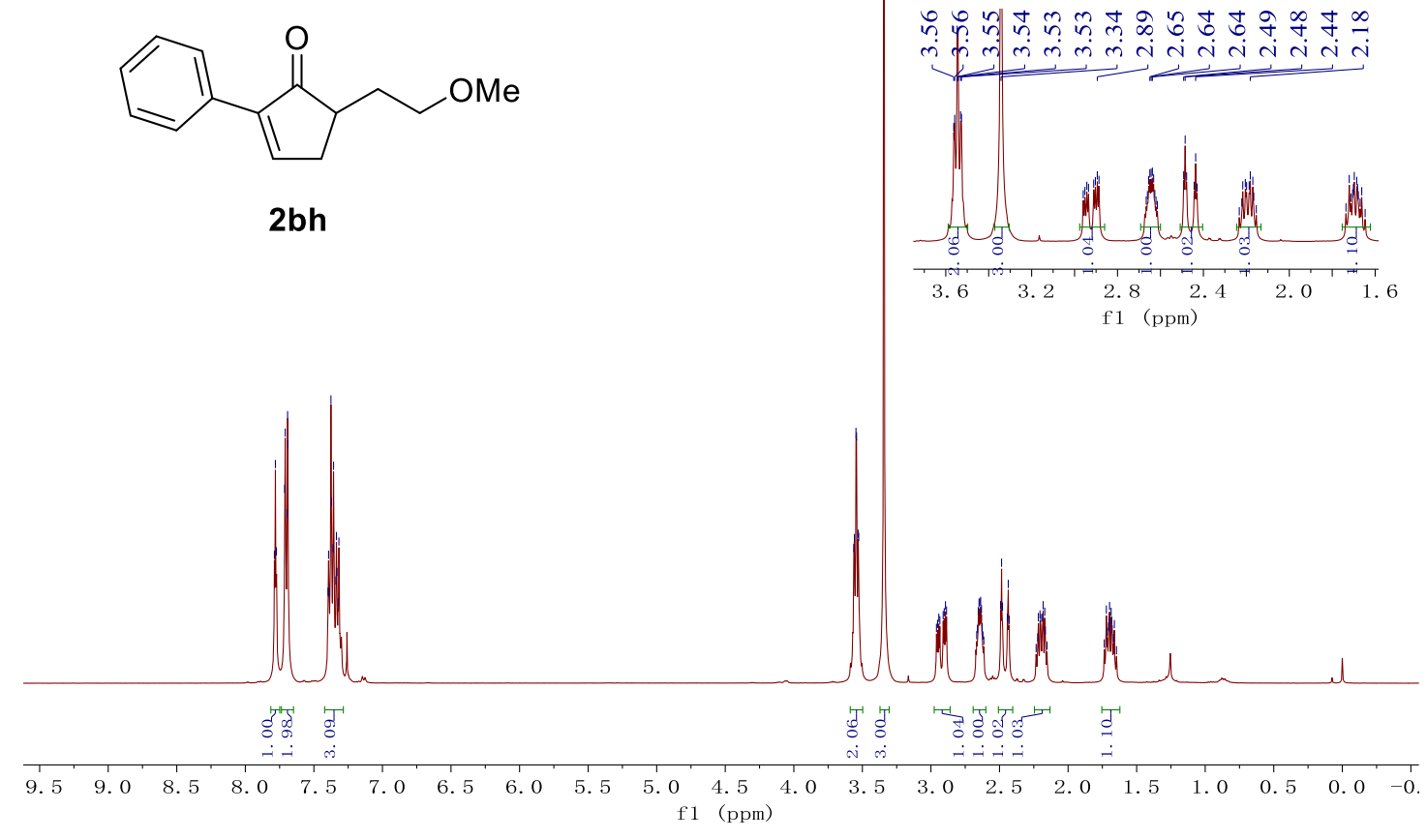<smiles>COCCC1CC=C(c2ccccc2)C1=O</smiles>

$2 \mathrm{bh}$

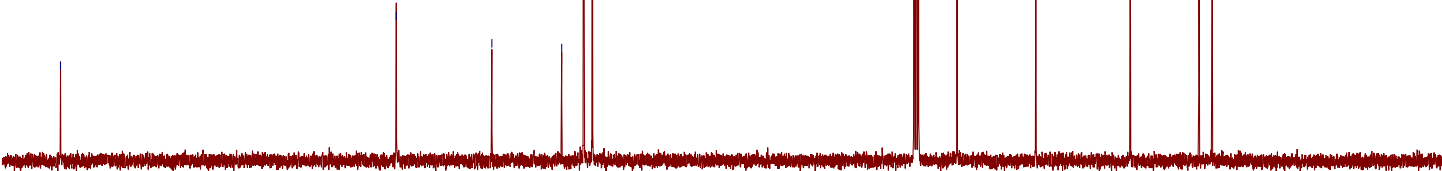




\section{5-benzyl-2-phenylcyclopent-2-en-1-one (2bi)}

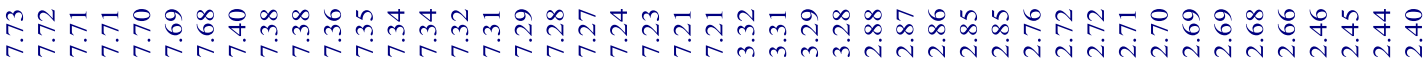
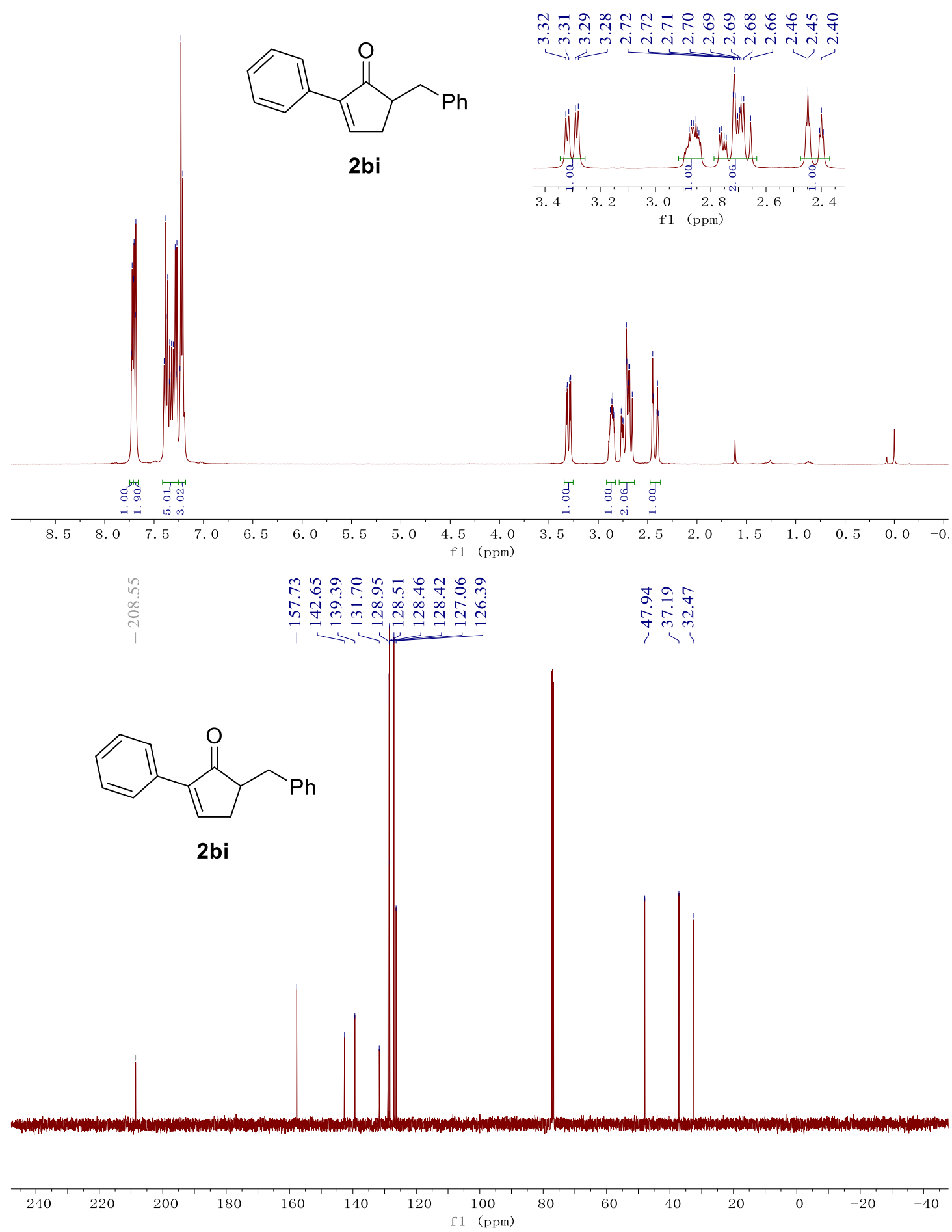
2,5-diphenylcyclopent-2-en-1-one (2ca)

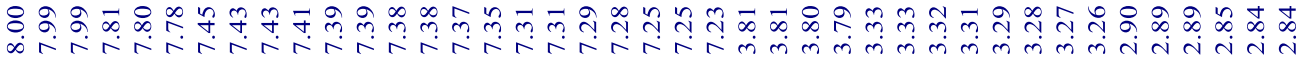<smiles>O=C1C(c2ccccc2)=CCC1c1ccccc1</smiles>

2ca

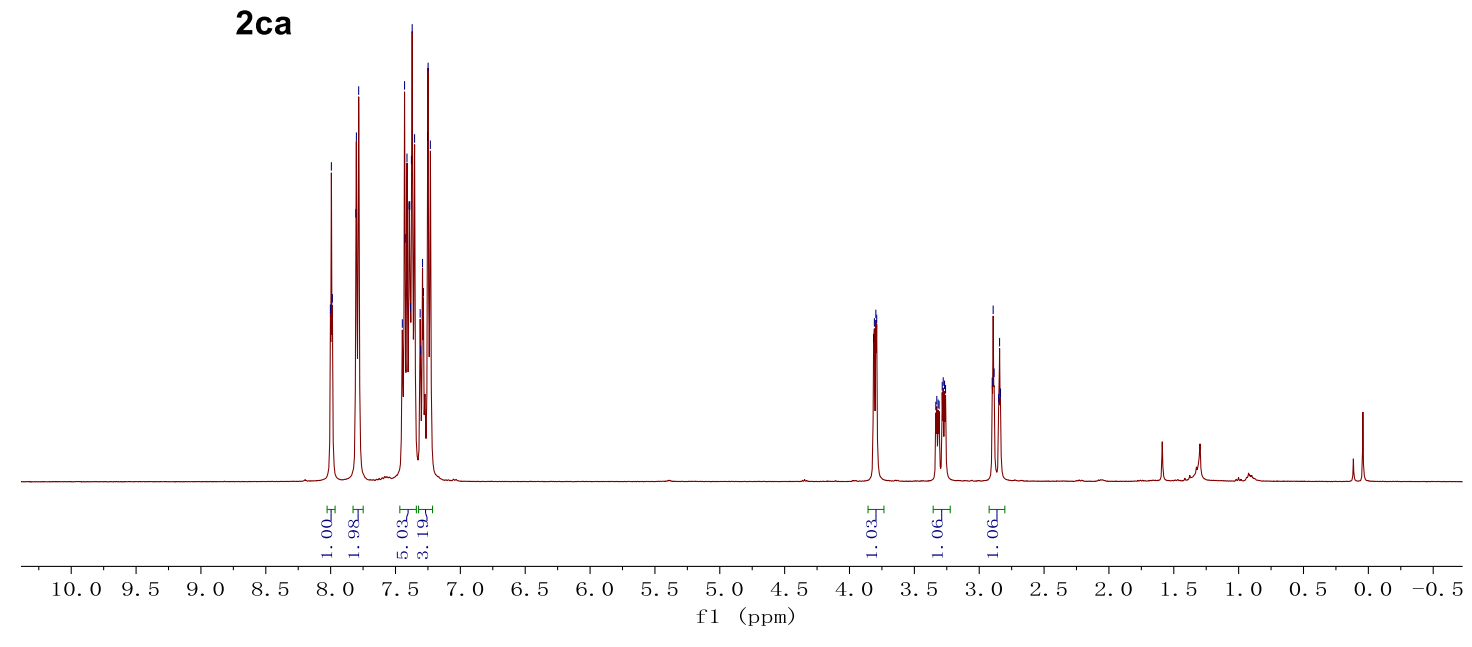

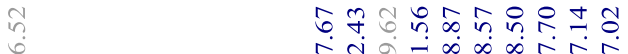

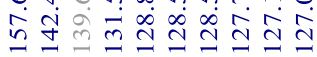

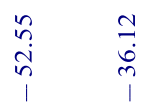<smiles>O=C1C(c2ccccc2)=CCC1c1ccccc1</smiles>

2ca

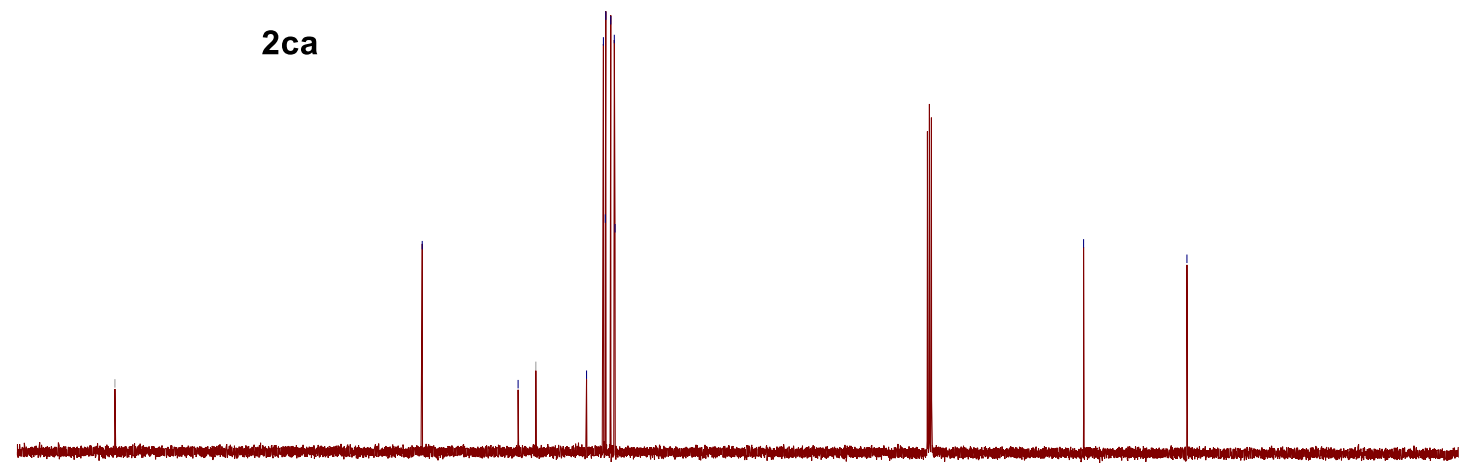

\begin{tabular}{llllllllllllllllllllllllllllllllll}
\hline 220 & 210 & 200 & 190 & 180 & 170 & 160 & 150 & 140 & 130 & 120 & 110 & 100 & 90 & 80 & 70 & 60 & 50 & 40 & 30 & 20 & 10 & 0
\end{tabular} f1 (ppm) 
2-phenyl-5-(o-tolyl)cyclopent-2-en-1-one (2cb)

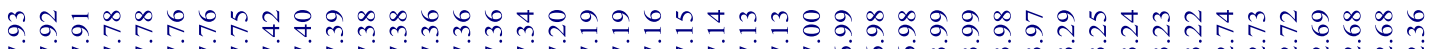

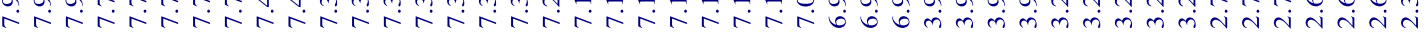

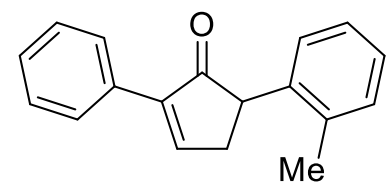

$2 \mathrm{cb}$
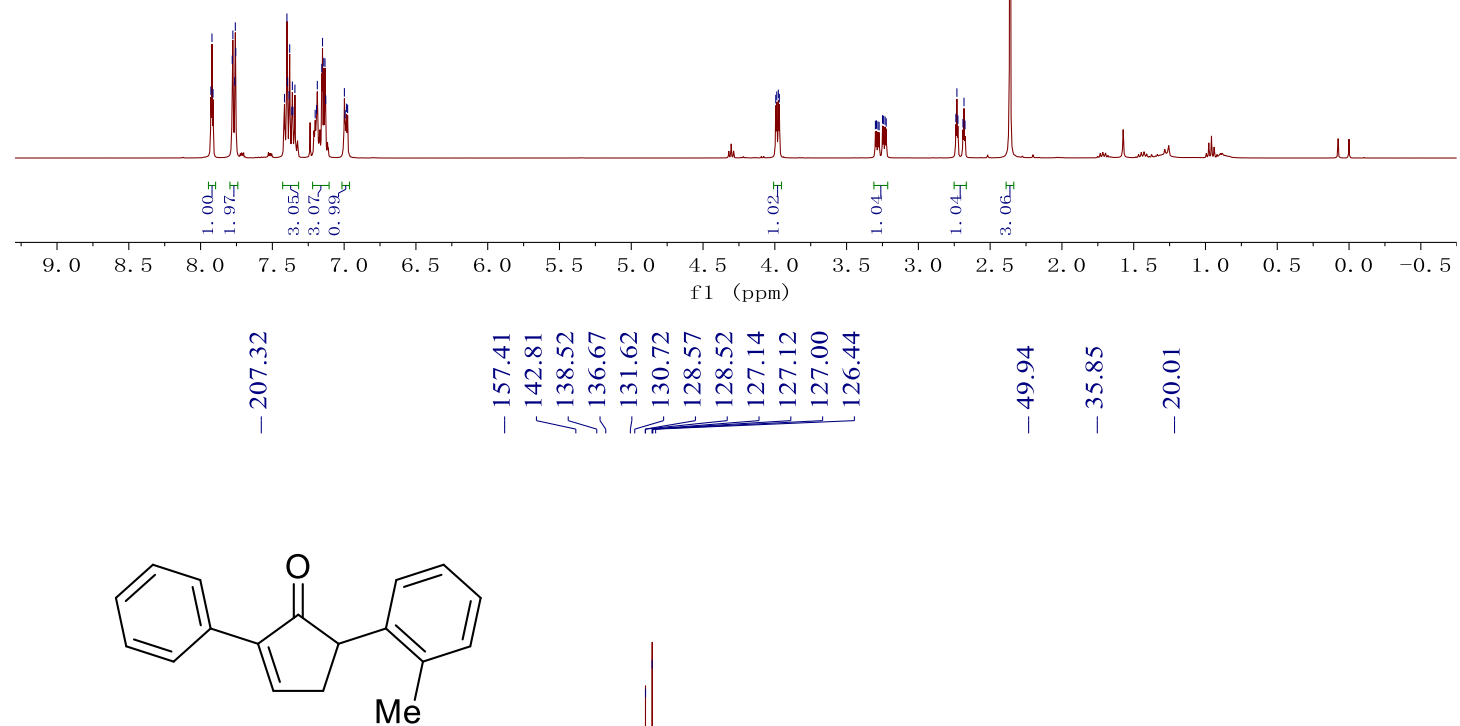

$2 c b$

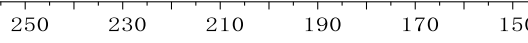

110 
2-phenyl-5-(thiophen-3-yl)cyclopent-2-en-1-one (2cc)

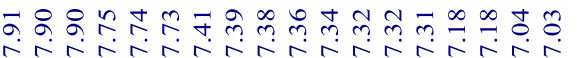

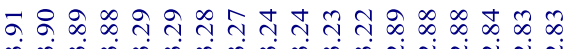

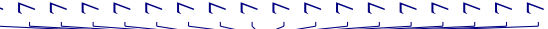

mm mm miri idiri<smiles>O=C1C(c2ccccc2)=CCC1c1ccsc1</smiles>

2cc

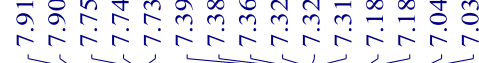

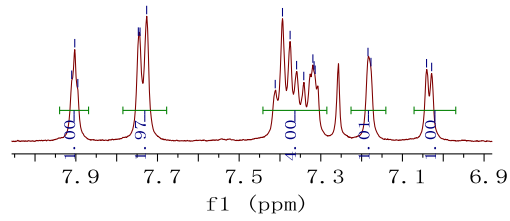

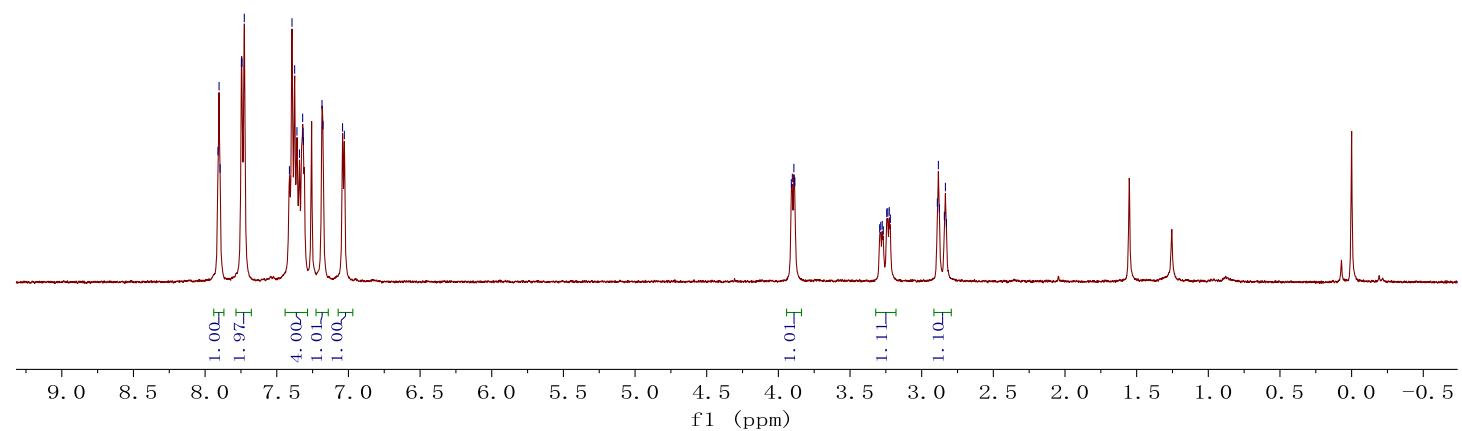

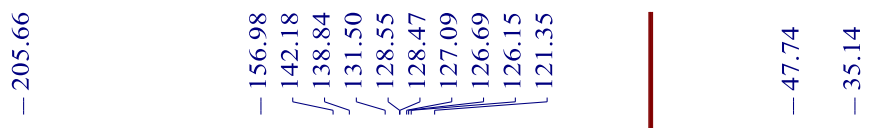<smiles>O=C1C(c2ccccc2)=CCC1c1ccsc1</smiles>

$2 c c$

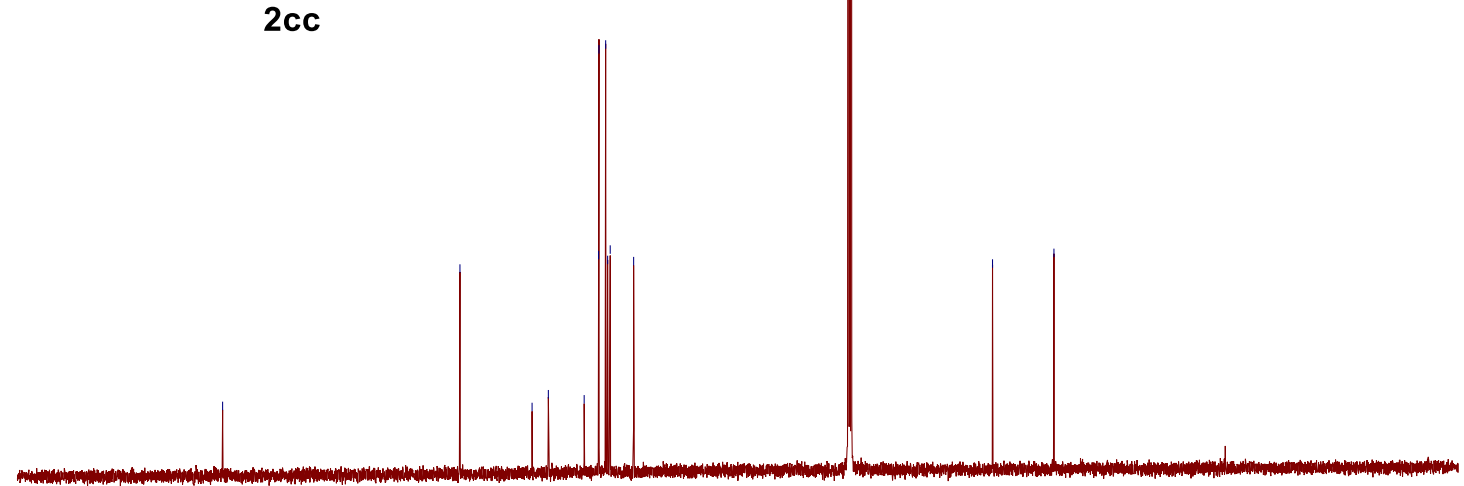

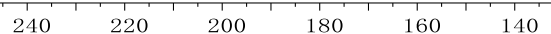
1 (ppm) 
2-benzyl-5-methylcyclopent-2-en-1-one (2da)

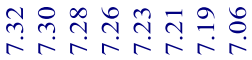

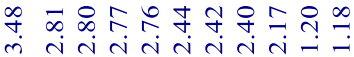

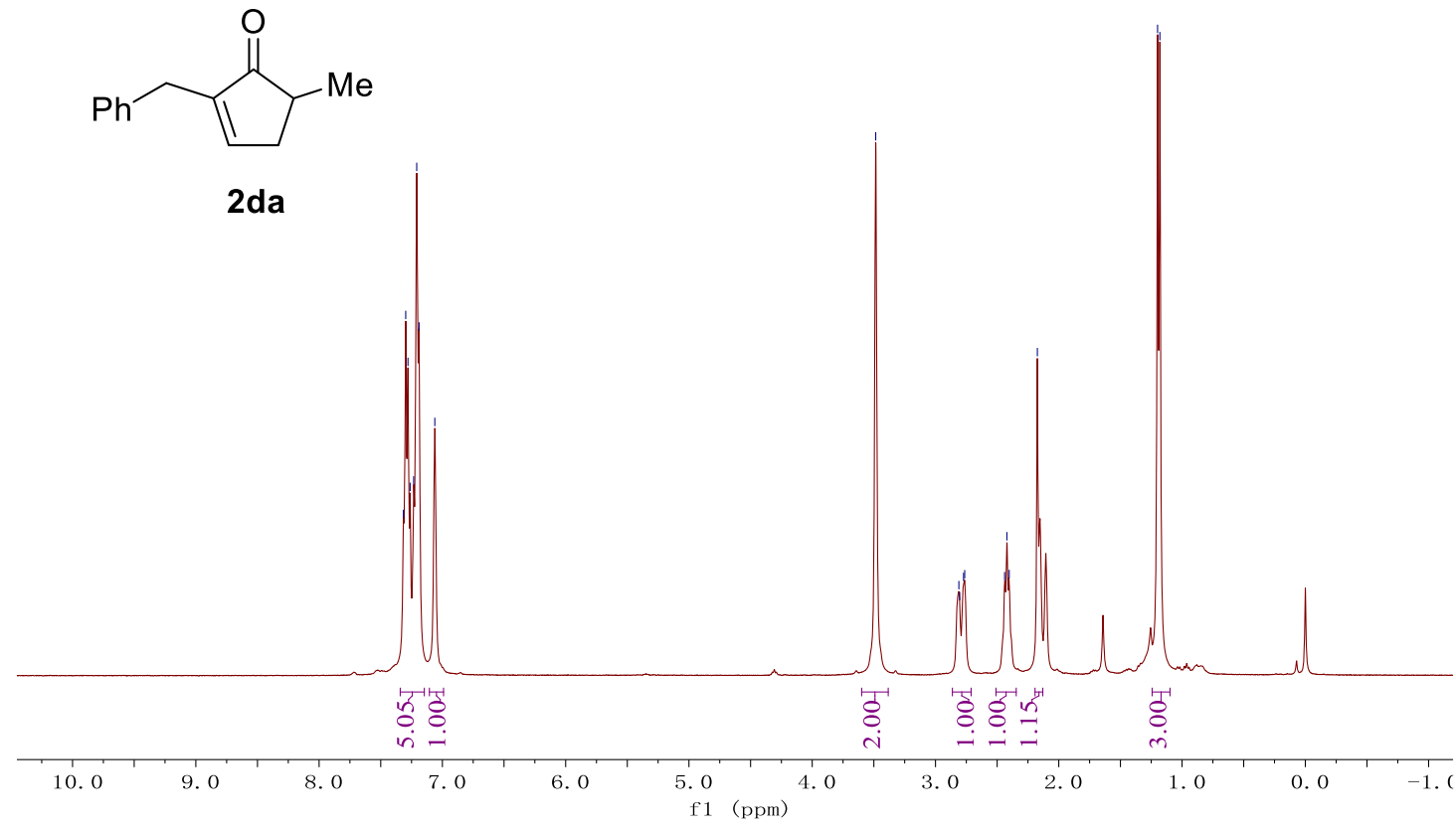

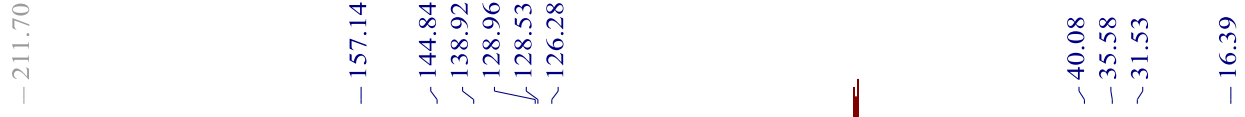<smiles>CC1CC=C(Cc2ccccc2)C1=O</smiles>

2da

$\begin{array}{lllllllllllllllllllllll}220 & 210 & 200 & 190 & 180 & 170 & 160 & 150 & 140 & 130 & 120 & 110 & 100 & 90 & 80 & 70 & 60 & 50 & 40 & 30 & 20 & 10 & 0\end{array}$ f1 (ppm) 


\section{2-butyl-5-methylcyclopent-2-en-1-one (2db)}

亲

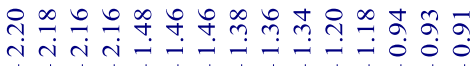

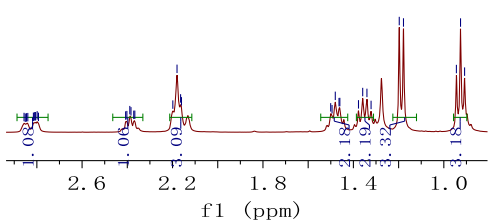<smiles>CCCCC1=CCC(C)C1=O</smiles>

$2 \mathrm{db}$

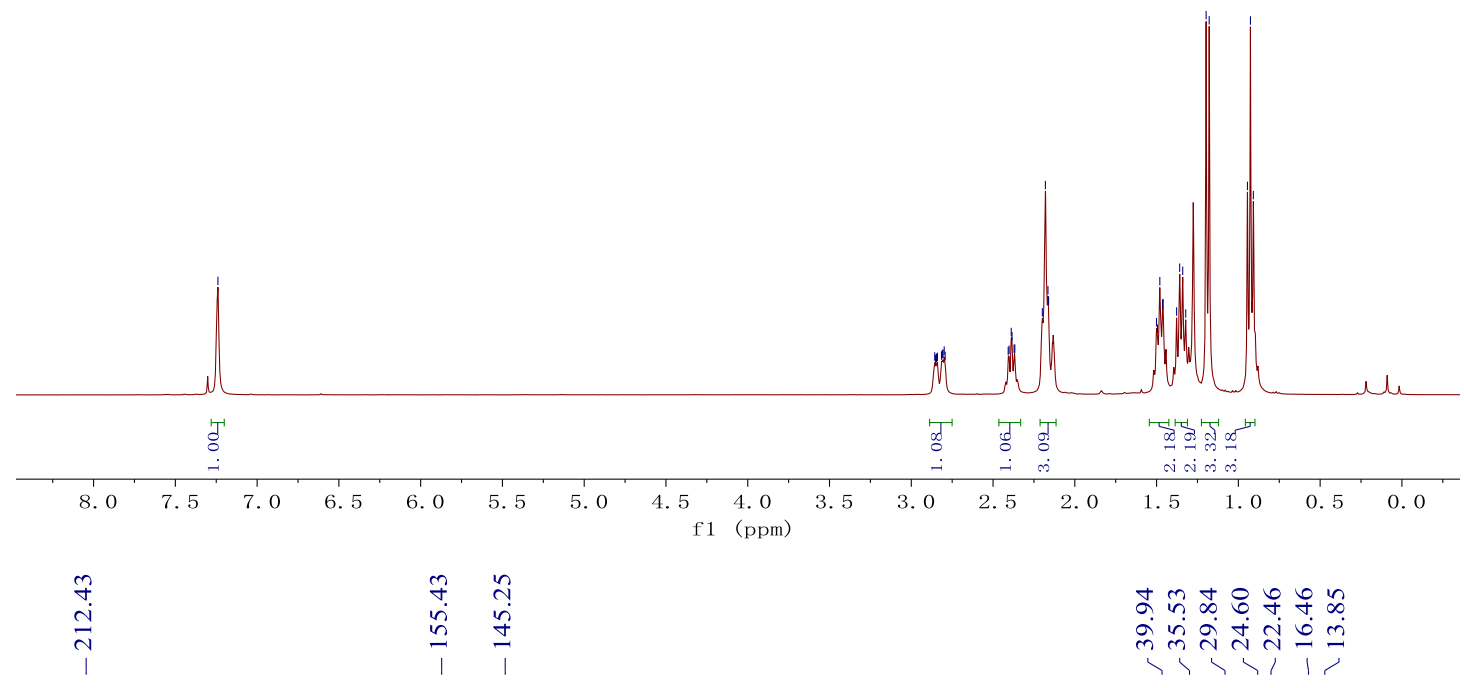<smiles>CCCCC1=CCC(C)C1=O</smiles>

$2 \mathrm{db}$

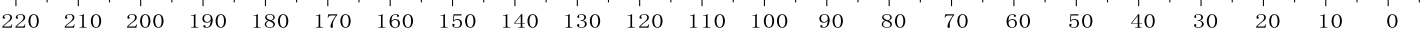
f1 (ppm) 


\section{2-isopropyl-5-methylcyclopent-2-en-1-one (2dc)}

$\stackrel{2}{i}$

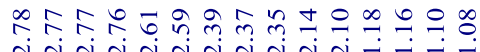

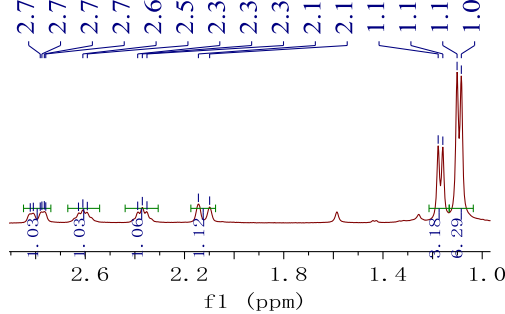

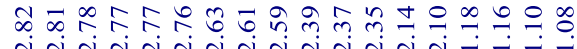

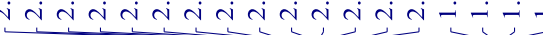<smiles>CC(C)C1=CCC(C)C1=O</smiles>

2dc

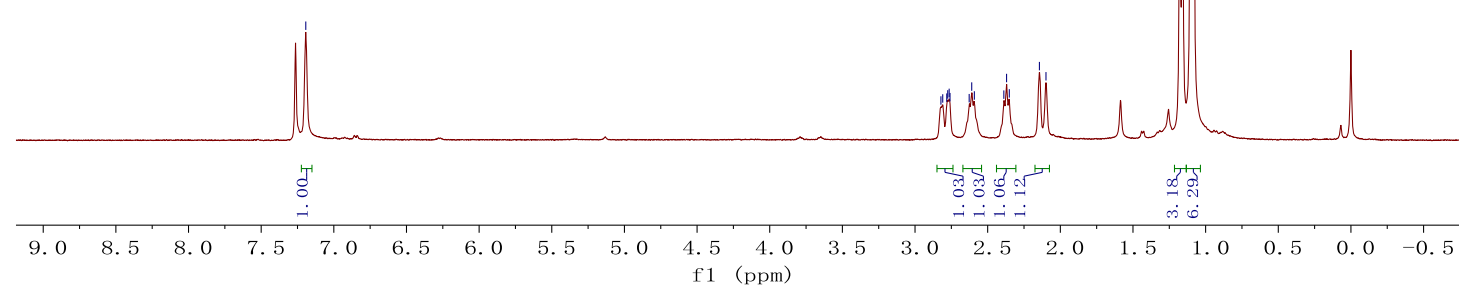
$\underset{\substack{\text { i } \\ \text { i }}}{1}$

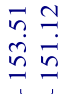

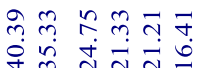
की<smiles>CC(C)C1=CCC(C)C1=O</smiles>

2dc

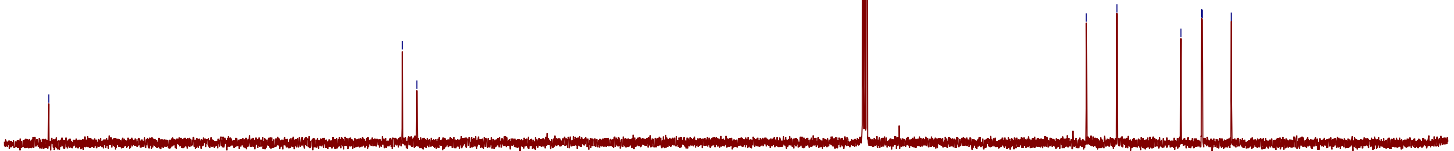

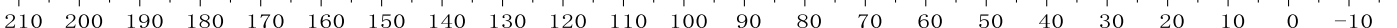
f1 (ppm) 


\section{3-methyl-[1,1'-bi(cyclopentan)]-5-en-2-one (2dd)}

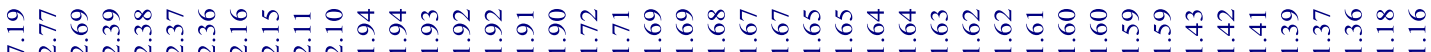
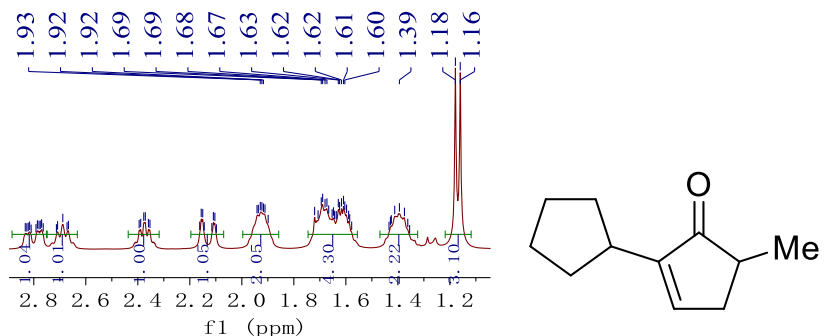

2dd
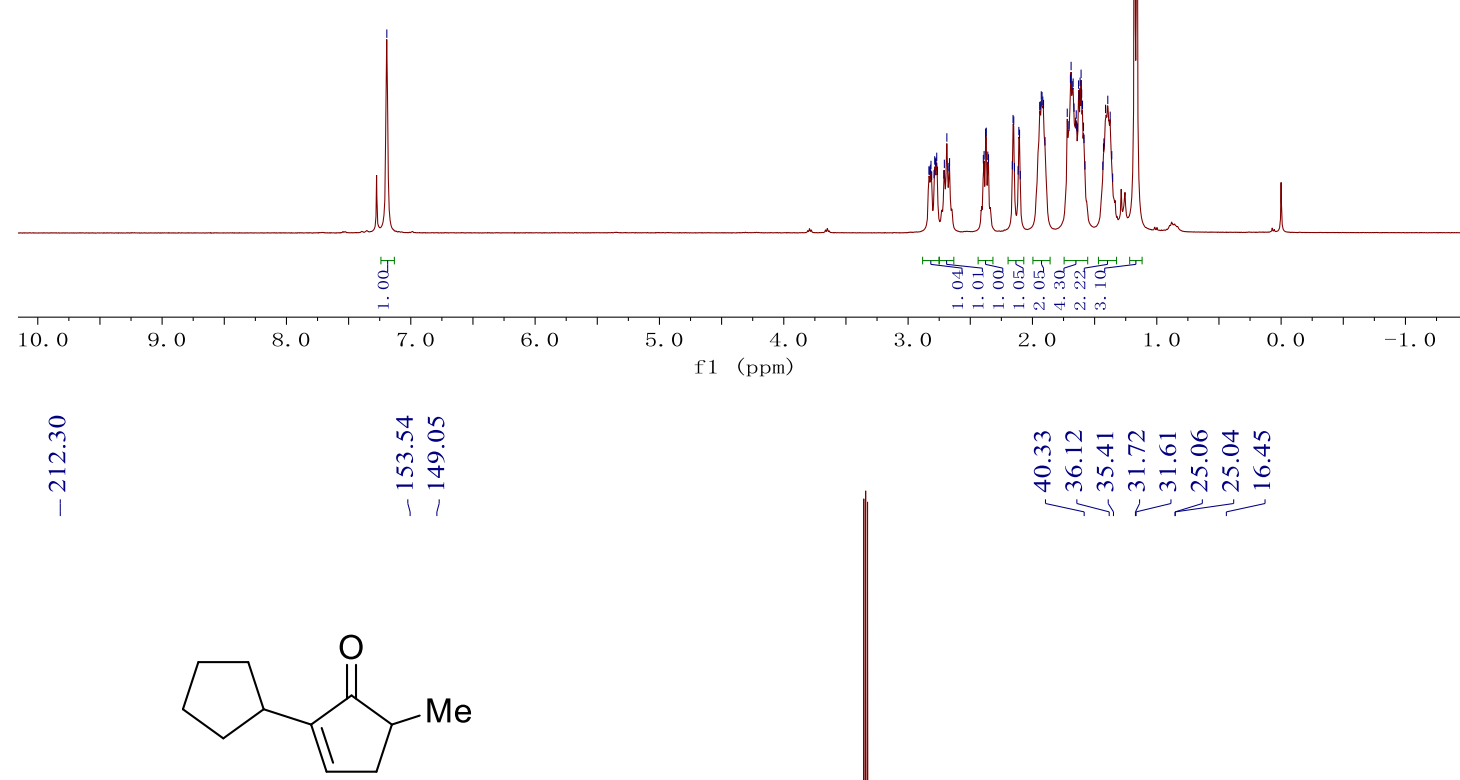

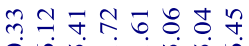

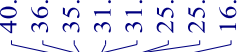

2dd 


\section{2-cyclohexyl-5-methylcyclopent-2-en-1-one (2de)}

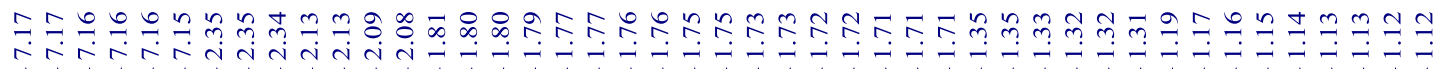
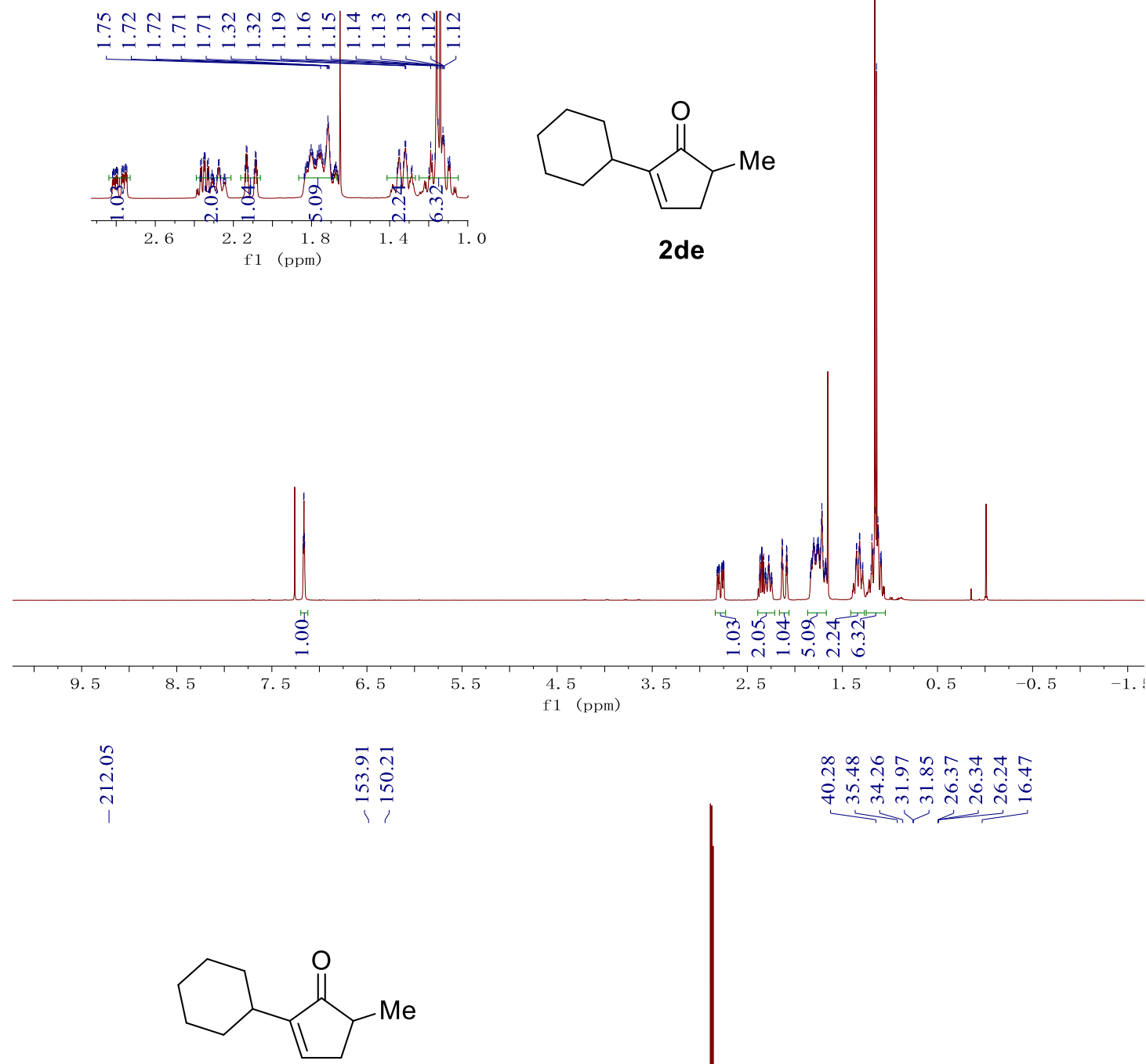

2de

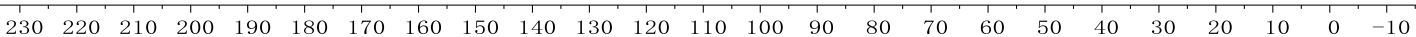
f1 (ppm) 
2-(cyclohex-1-en-1-yl)-5-methylcyclopent-2-en-1-one (2ea)

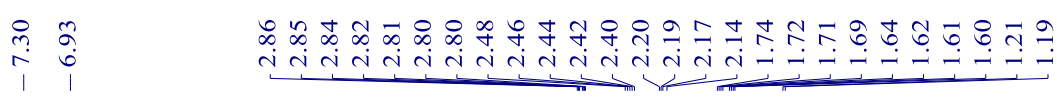

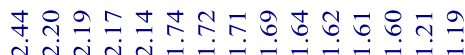

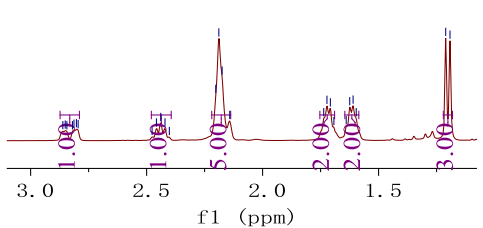<smiles>CC1CC=C(C2=CCCCC2)C1=O</smiles>

2ea

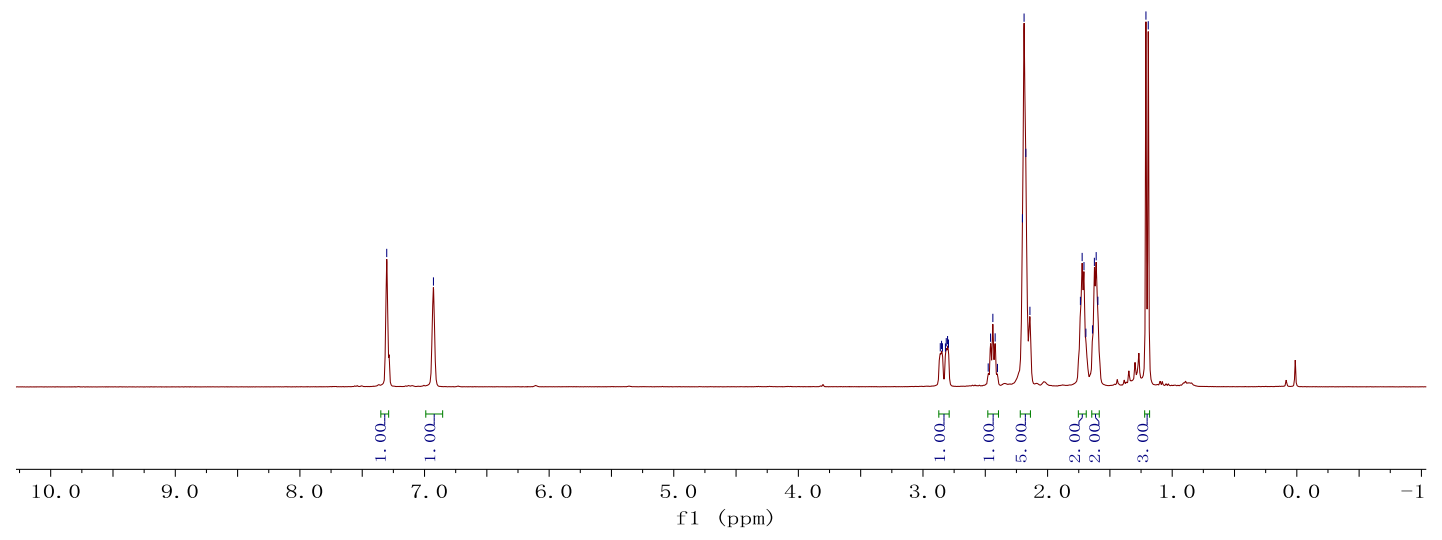

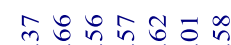

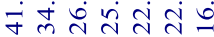<smiles>CC1CC=C(C2=CCCCC2)C1=O</smiles>

2ea

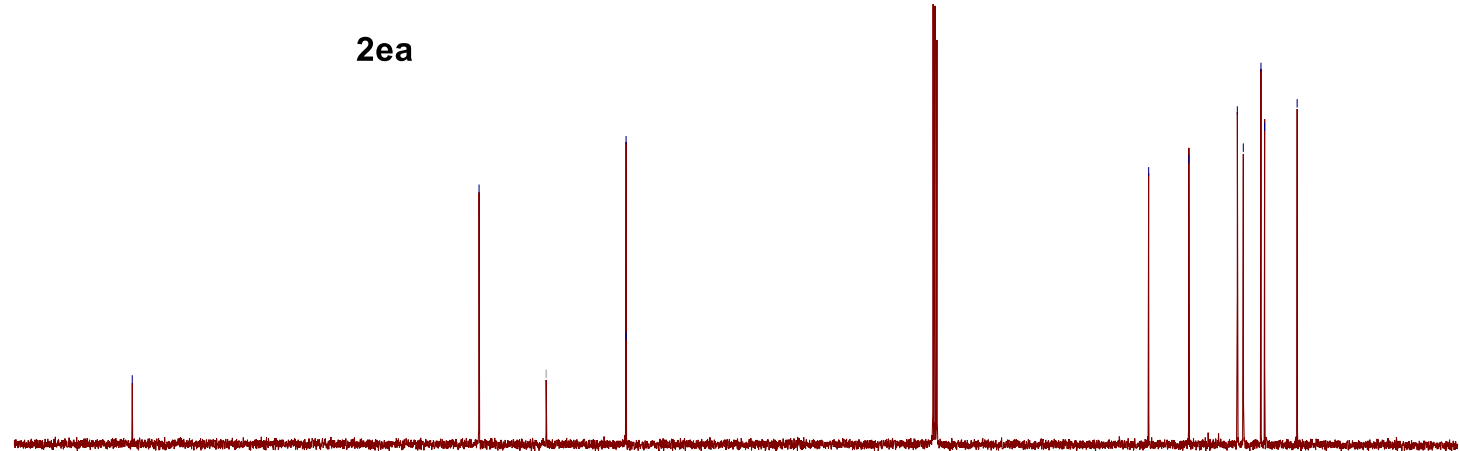

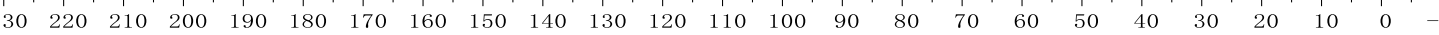
f1 (ppm) 


\section{5-methyl-2-(oct-1-yn-1-yl)cyclopent-2-en-1-one (2fa)}

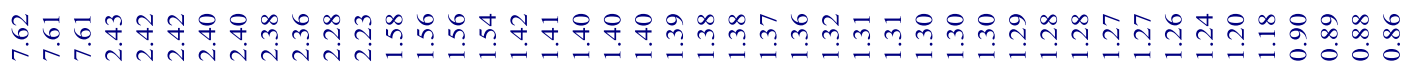

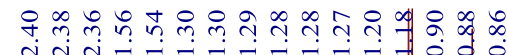
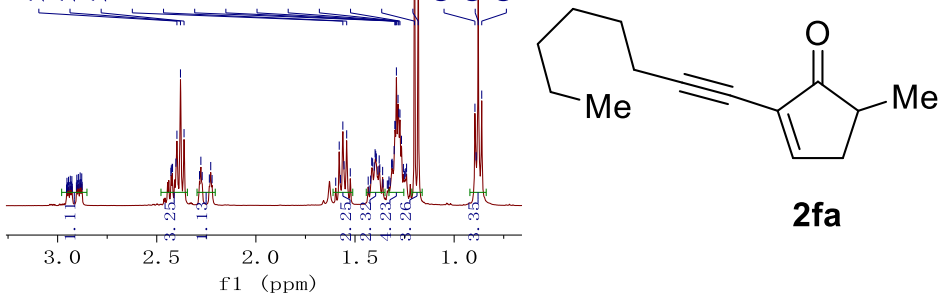

$2 \mathrm{fa}$

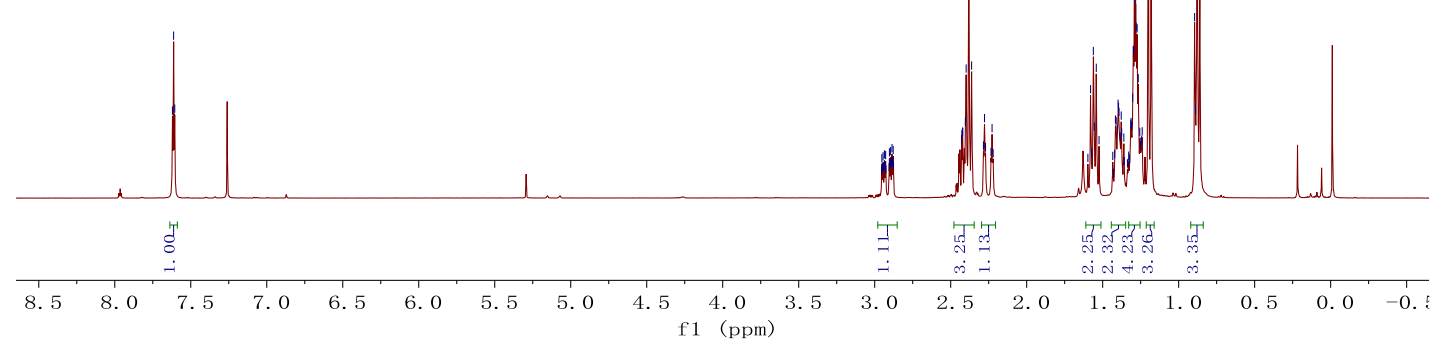

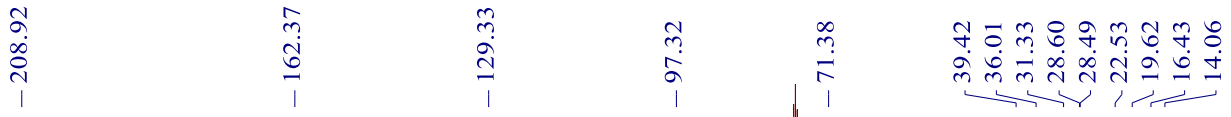

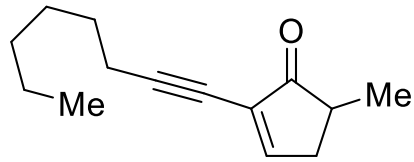

$2 \mathrm{fa}$

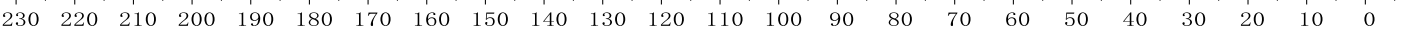
f1 (ppm) 


\section{4,5-dimethyl-2-phenylcyclopent-2-en-1-one (2ga)}

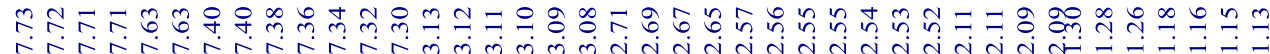

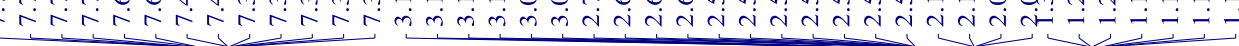

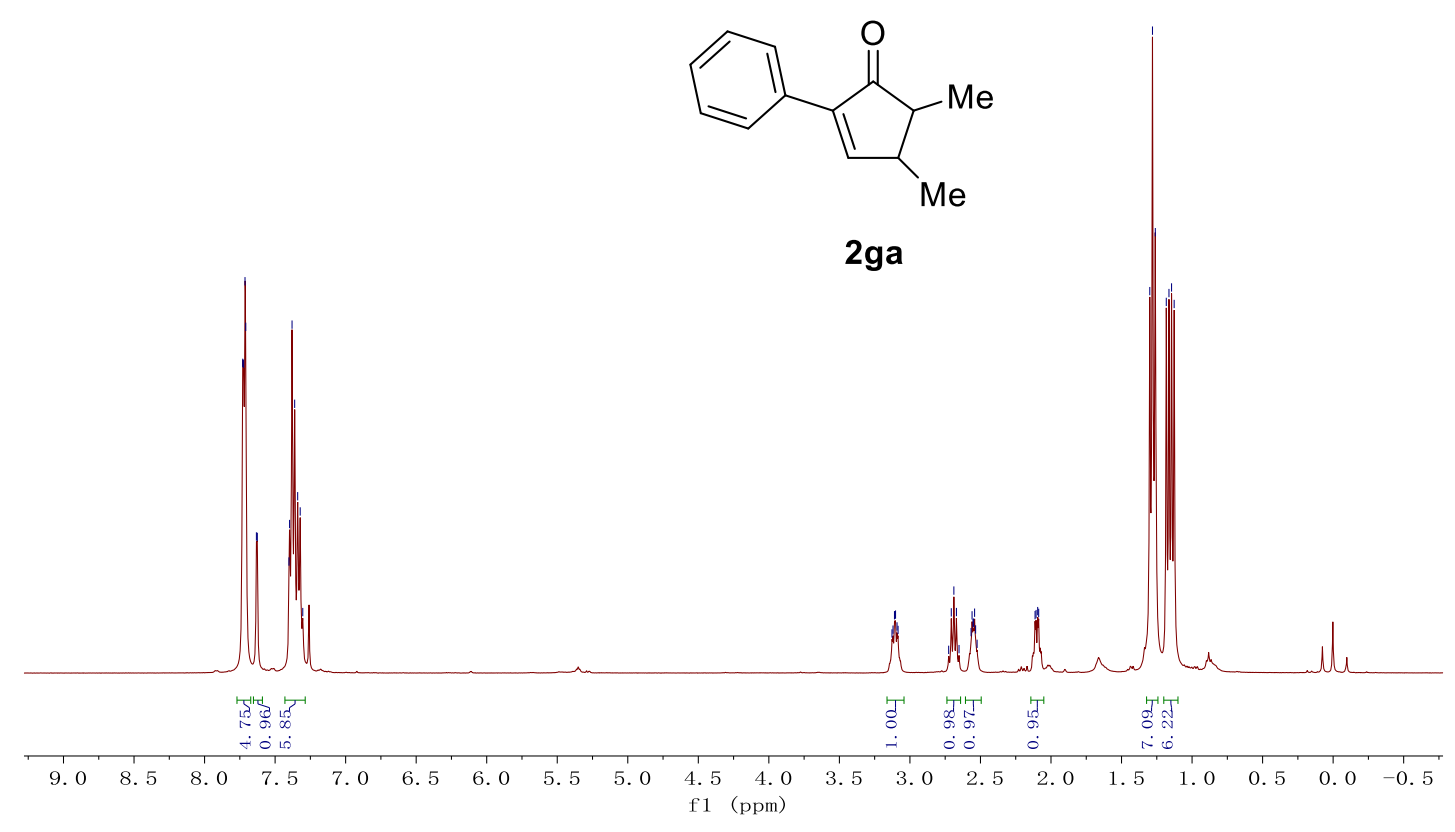

2ga

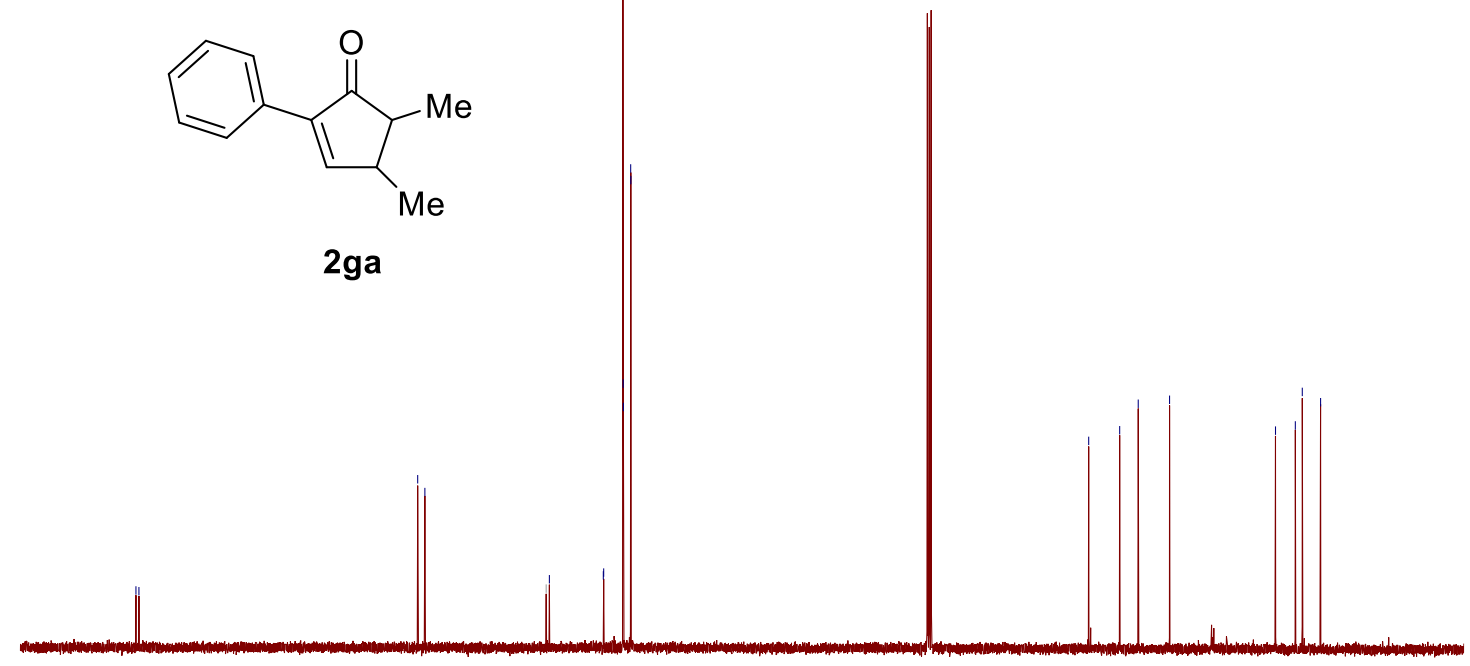

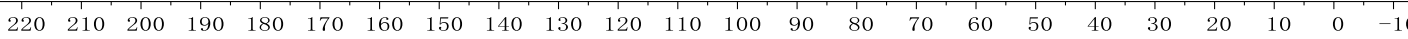
f1 (ppm) 


\section{5-isopropyl-4-methyl-2-phenylcyclopent-2-en-1-one (2gb)}

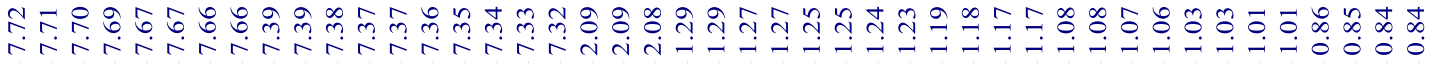<smiles>CC1=CC(c2ccccc2)=C(C(C)C)C1=O</smiles>

$$
2 \mathrm{gb}
$$

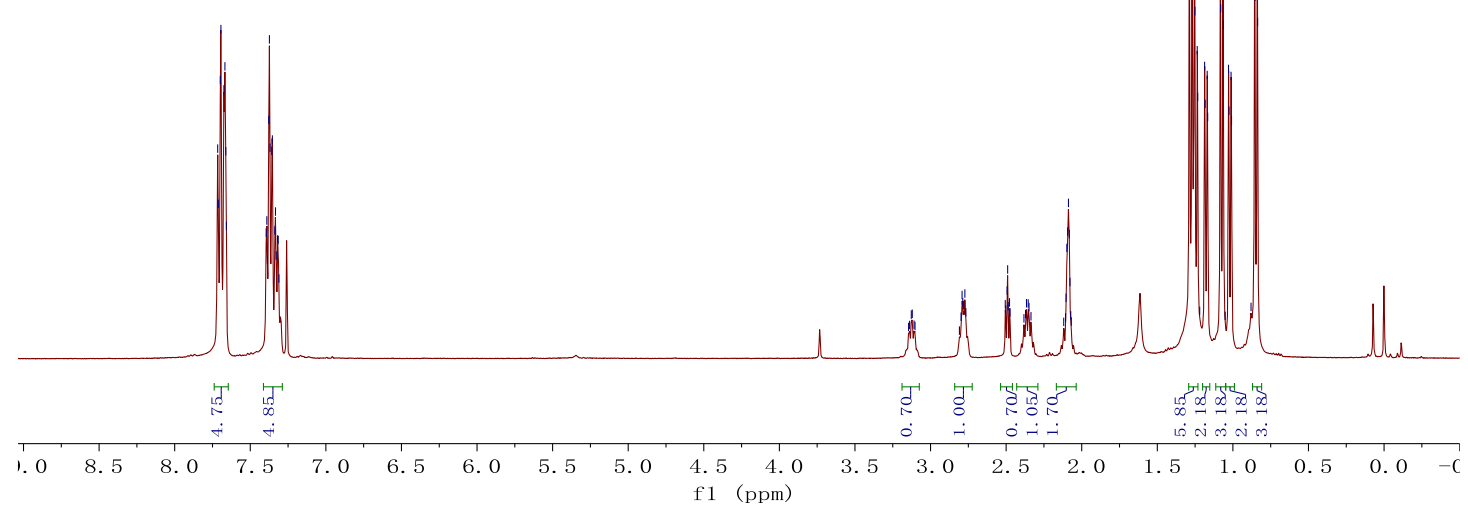

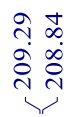

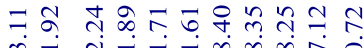

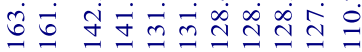

ミ

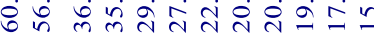<smiles>CC1=CC(c2ccccc2)=C(C(C)C)C1=O</smiles>

$2 \mathrm{gb}$ $\mathrm{f} 1(\mathrm{ppm})$ 


\section{5-methyl-2,4-diphenylcyclopent-2-en-1-one (2gc)}

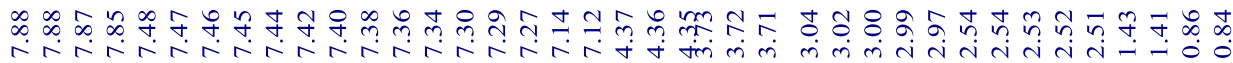<smiles>CC1C(=O)C(c2ccccc2)=CC1c1ccccc1</smiles>

$2 \mathrm{gc}$

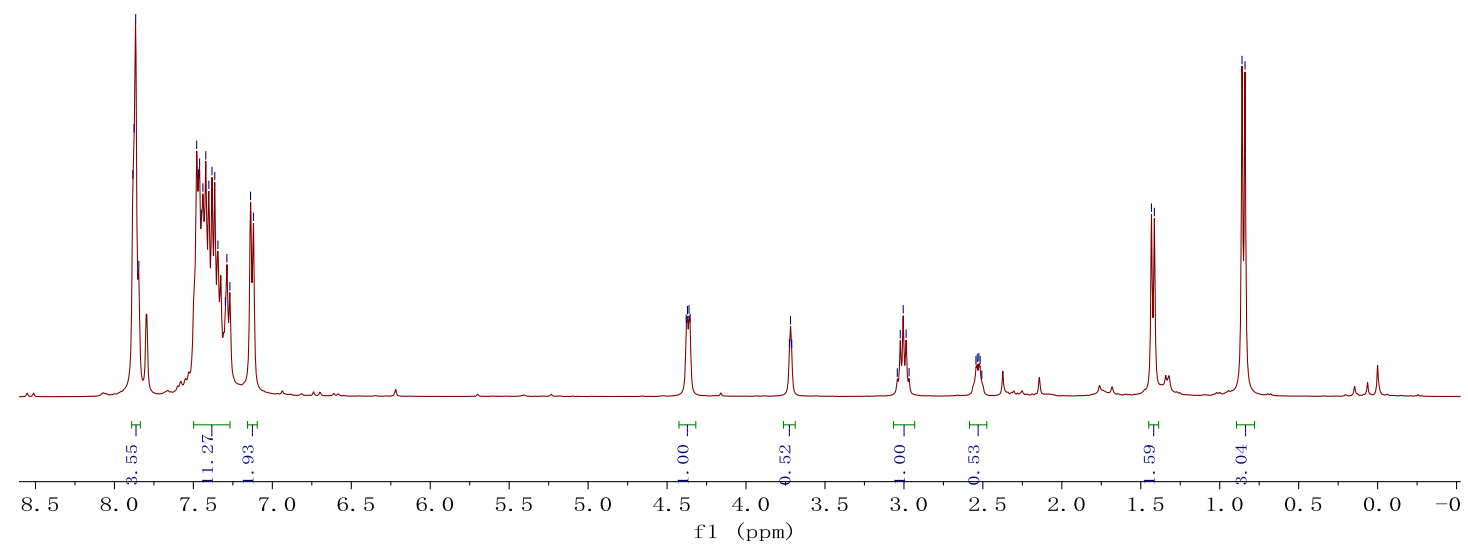
$\frac{2}{4}$

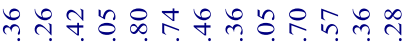
$\hat{l}$
ํㅜ요용
in $\sin$

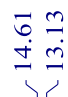<smiles>CC1C(=O)C(c2ccccc2)=CC1c1ccccc1</smiles>

$2 g c$

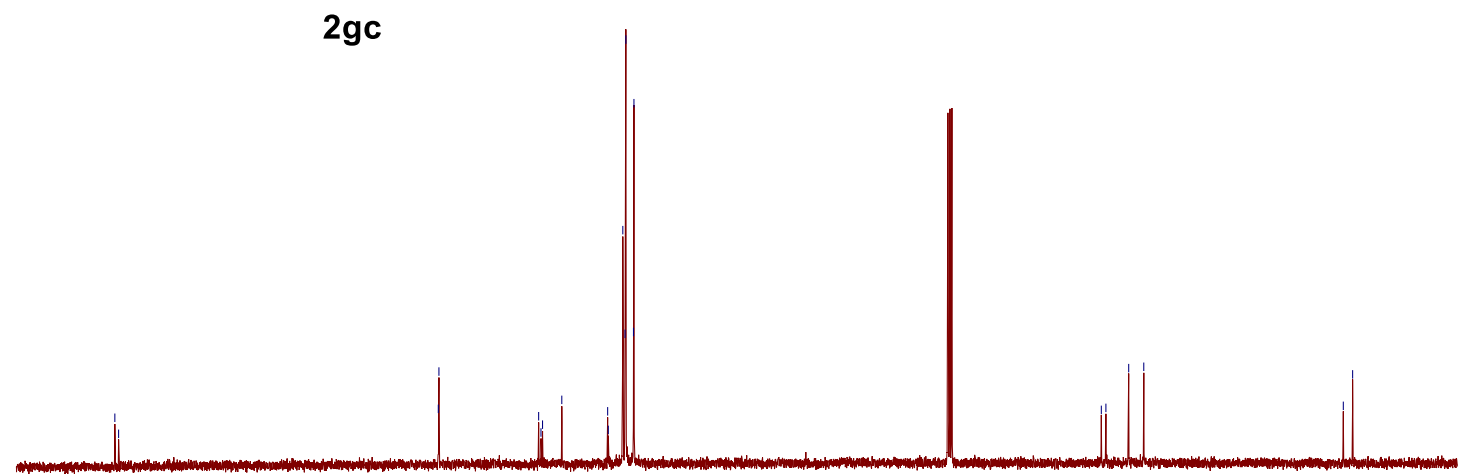

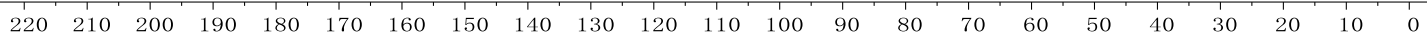
f1 (ppm) 


\section{5-methyl-2,3-diphenylcyclopent-2-en-1-one (2ha)}

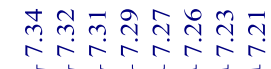

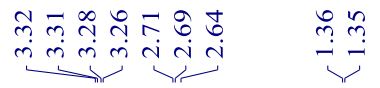

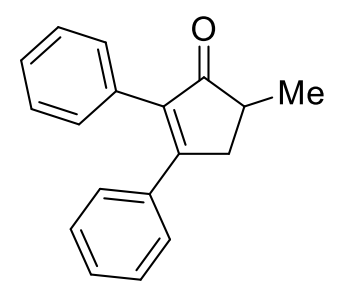

2ha
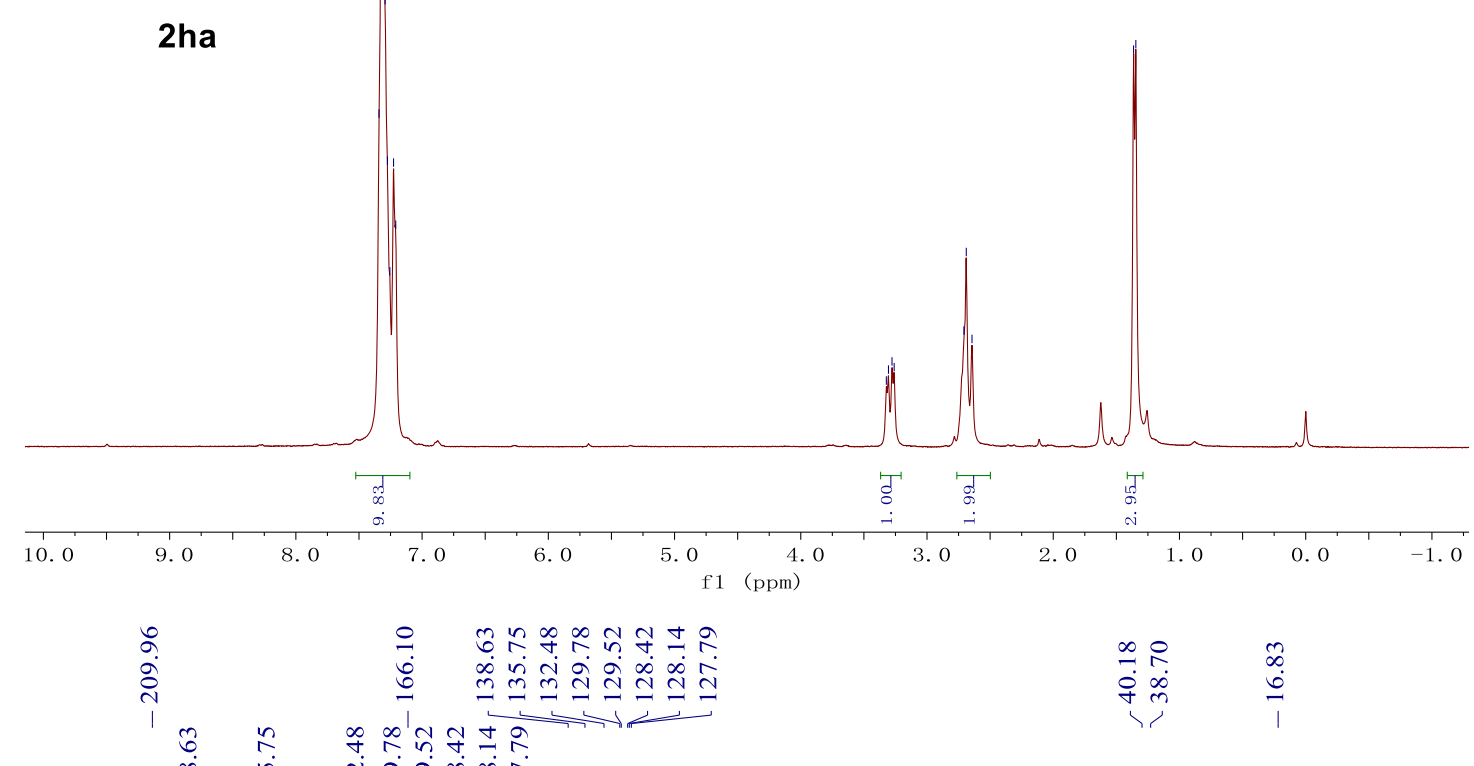

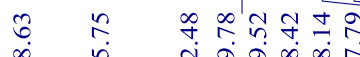

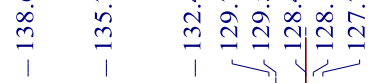

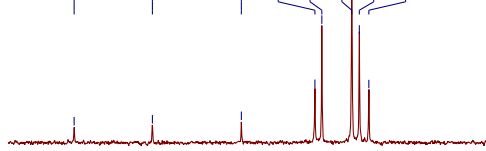

$\begin{array}{llllllllllll}140 & 138 & 136 & 134 & 132 & 130 & 128 & 126 & 124\end{array}$ f1 (ppm)

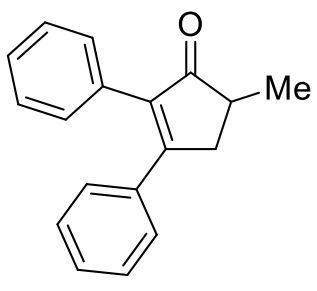

2ha

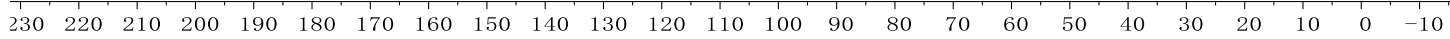
f1 (ppm) 


\section{4,4,5-trimethyl-2-phenylcyclopent-2-en-1-one (2ia)}

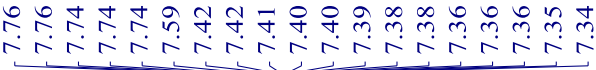

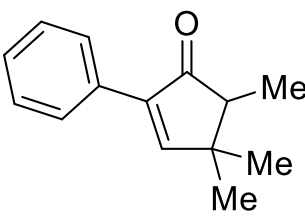

2ia

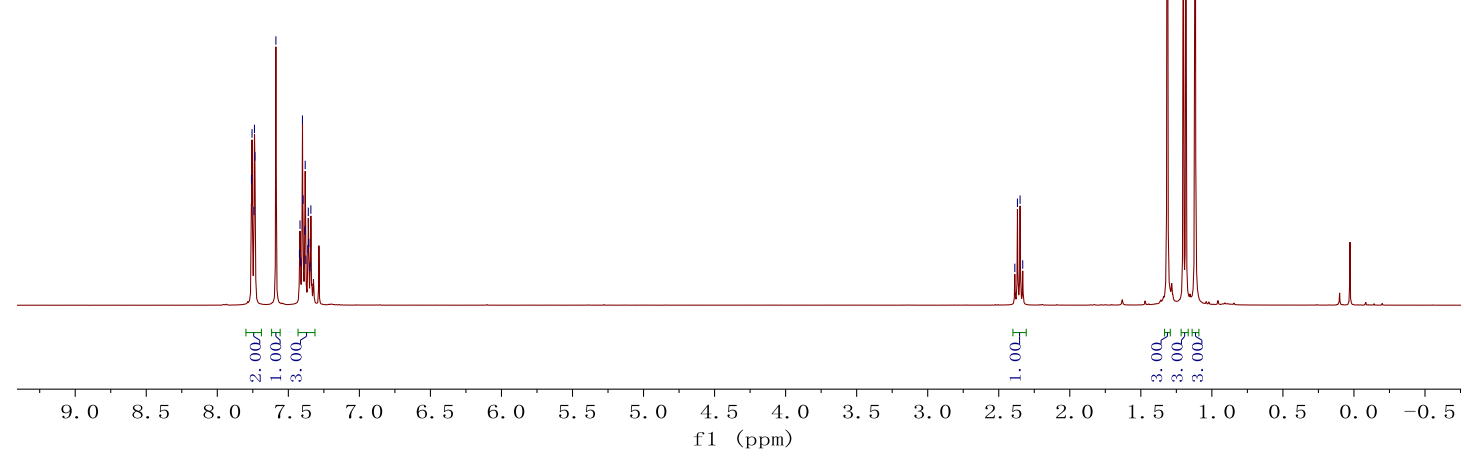

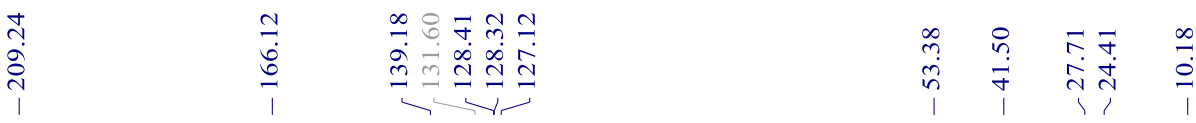<smiles>CC1C(=O)C(c2ccccc2)=CC1(C)C</smiles>

2ia

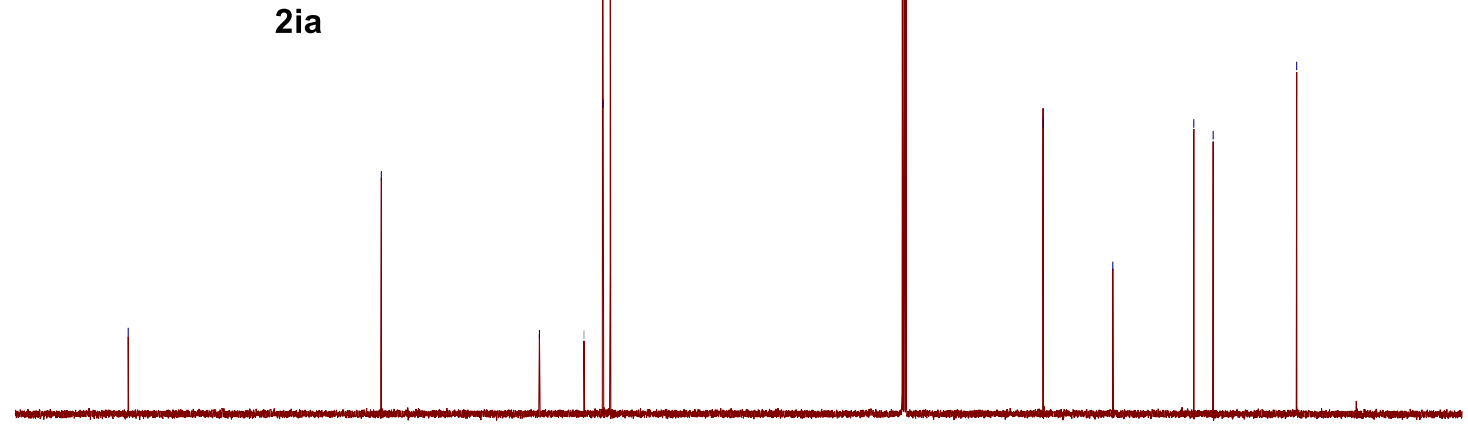

$\begin{array}{lllllllllllllllllllllllllllllllllll}220 & 210 & 200 & 190 & 180 & 170 & 160 & 150 & 140 & 130 & 120 & 110 & 100 & 90 & 80 & 70 & 60 & 50 & 40 & 30 & 20 & 10 & 0 & -10\end{array}$ f1 (ppm) 
methyl

2-((1R,4R)-3-((tert-butyldimethylsilyl)oxy)-4-methyl-2-phenylcyclopent-2-en-1-yl )acetate (5)

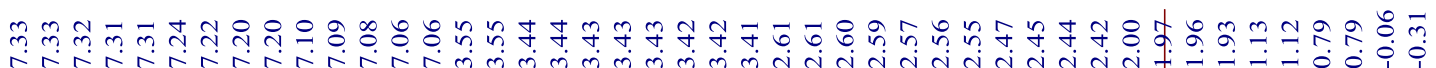

In $\mathrm{CDCl}_{3}$

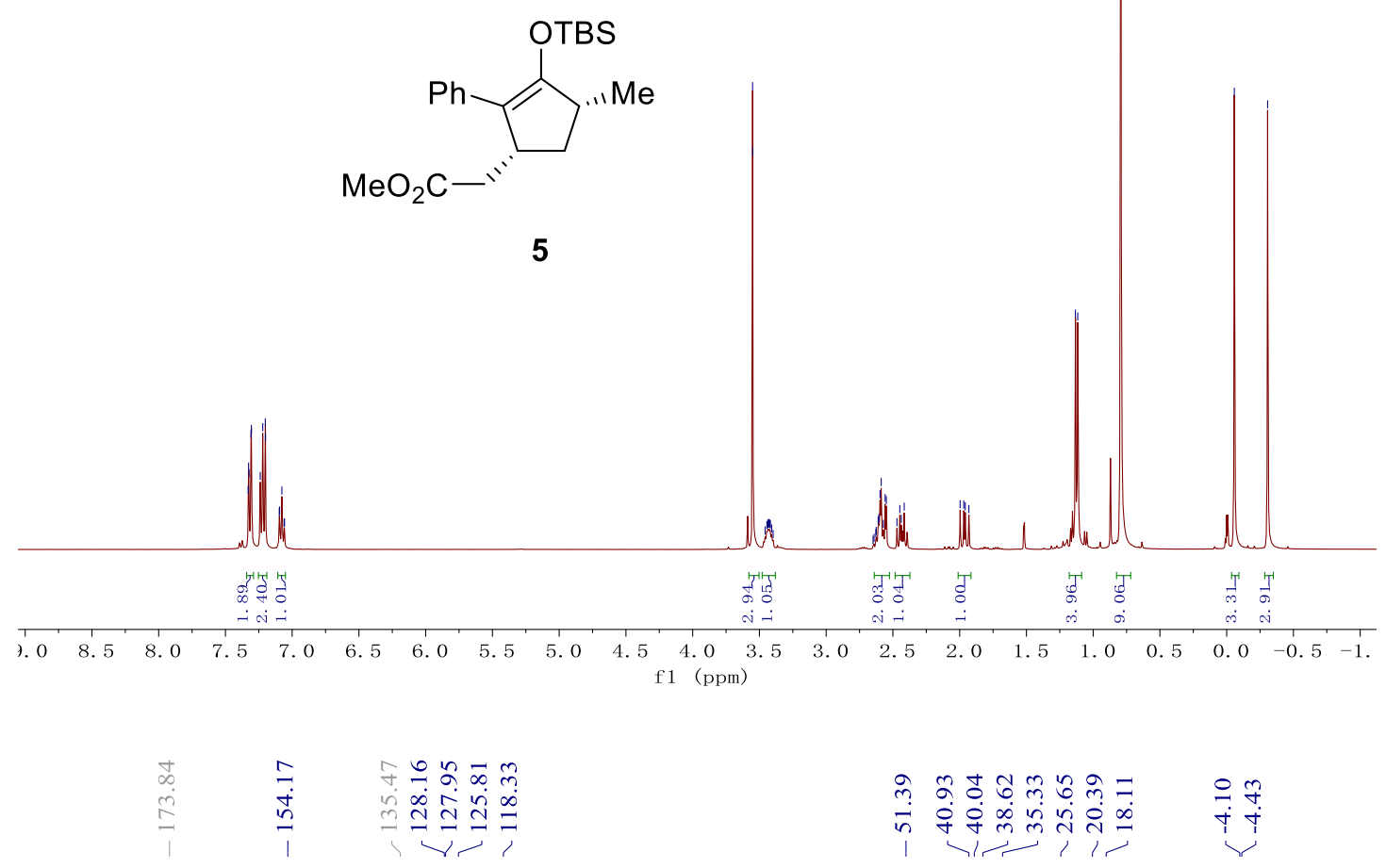<smiles>CC(=O)C[C@@H]1CC(C)C(OC(C)=O)=C1c1ccccc1</smiles>

5

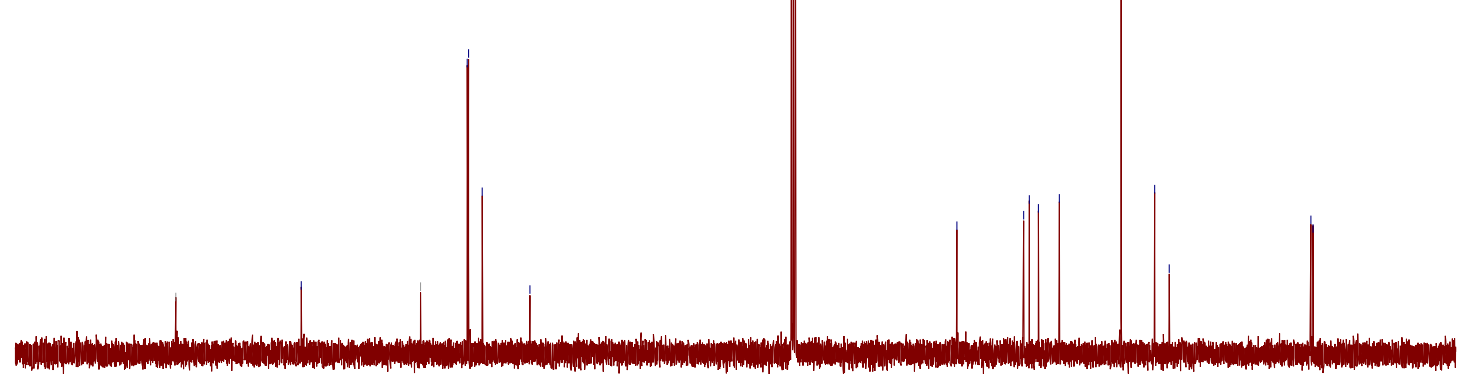

$\begin{array}{llllllllllllllllllllllllllllll}190 & 180 & 170 & 160 & 150 & 140 & 130 & 120 & 110 & 100 & 90 & 80 & 70 & 60 & 50 & 40 & 30 & 20 & 10 & 0 & -10 & -20\end{array}$ f1 (ppm) 


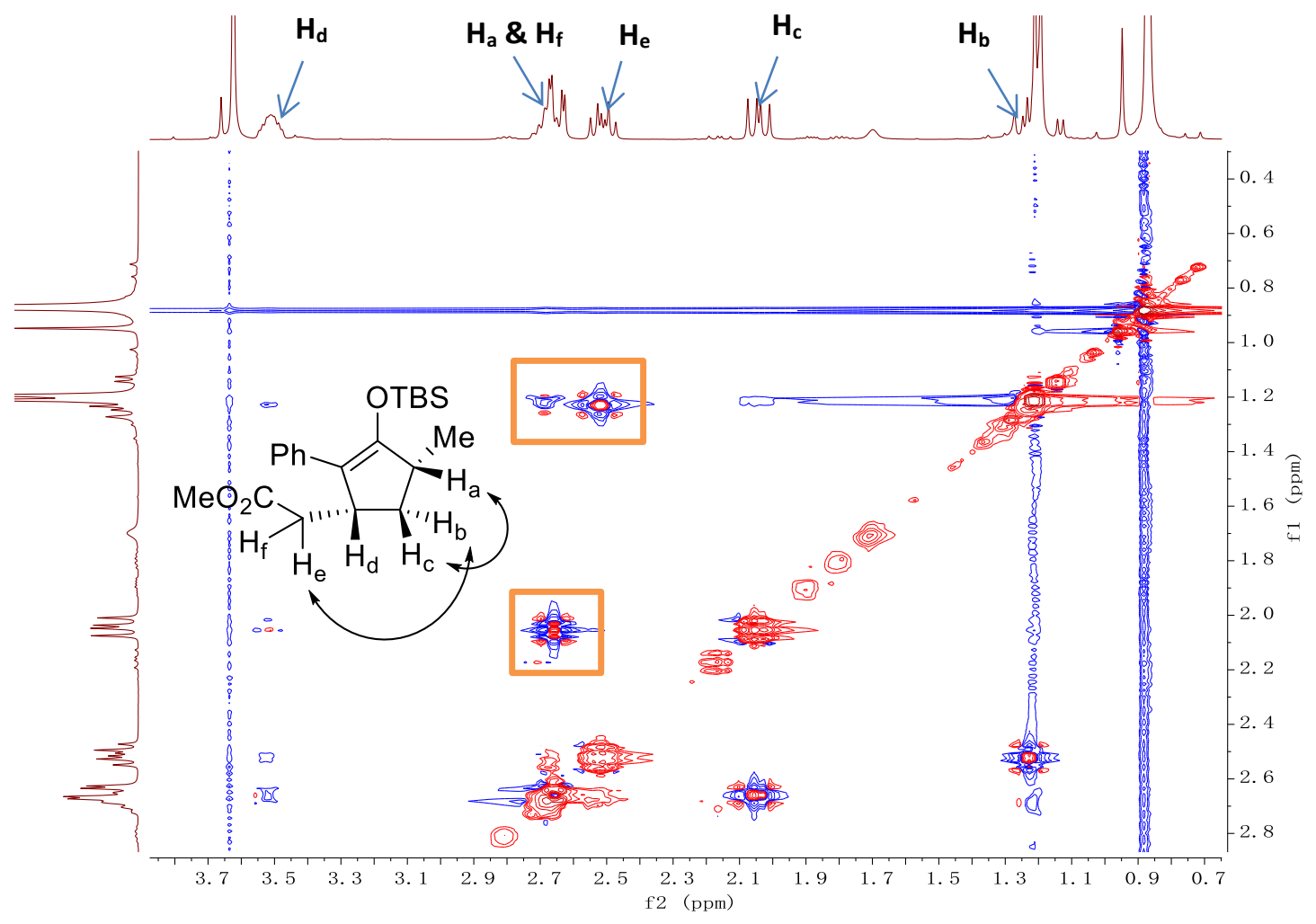




\section{In $\mathbf{C}_{6} \mathbf{D}_{6}$}
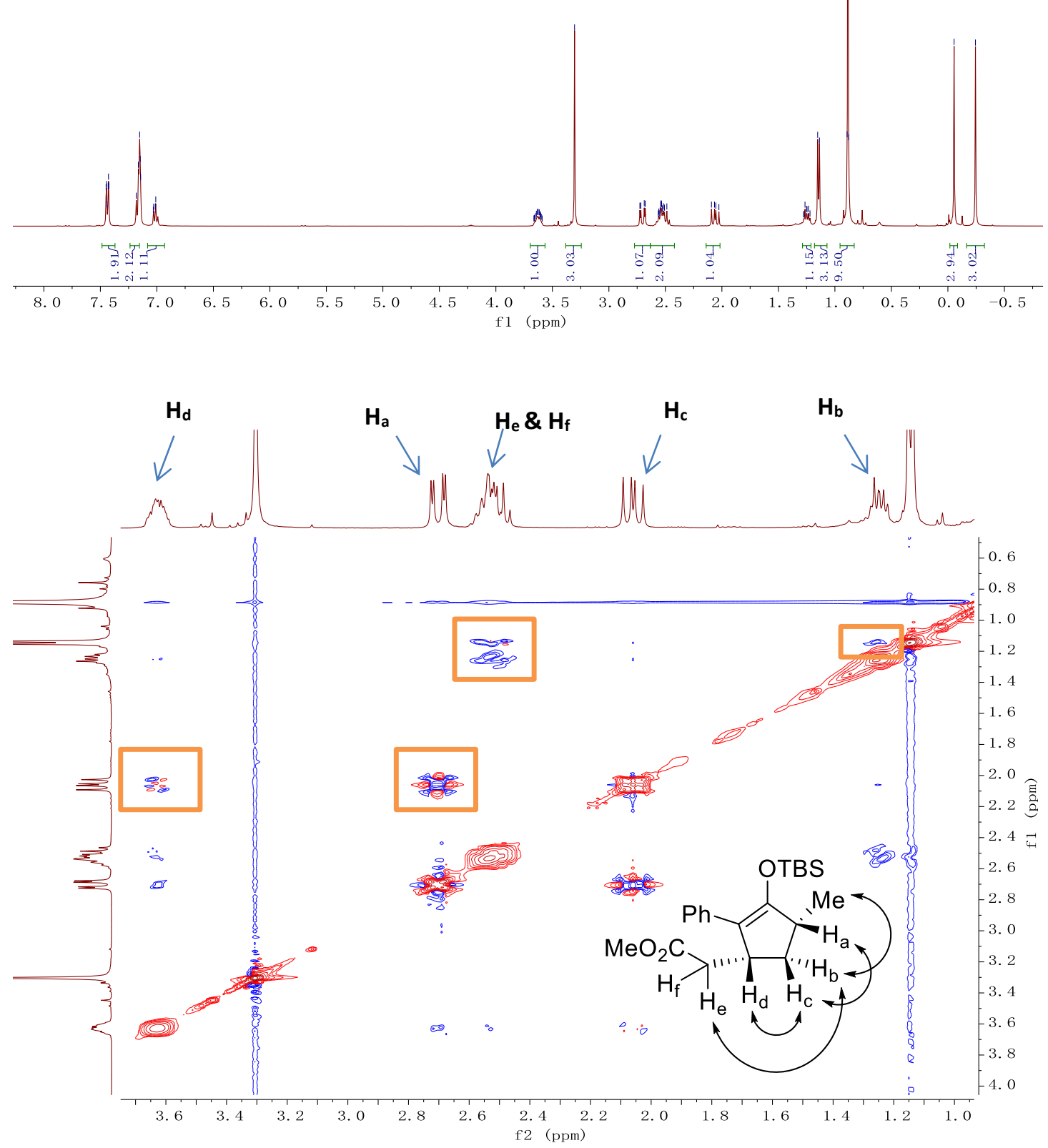


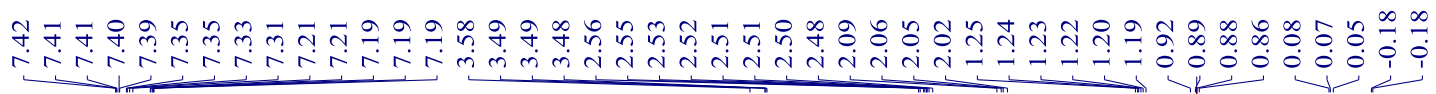

In $\mathrm{CD}_{3} \mathrm{CN}$
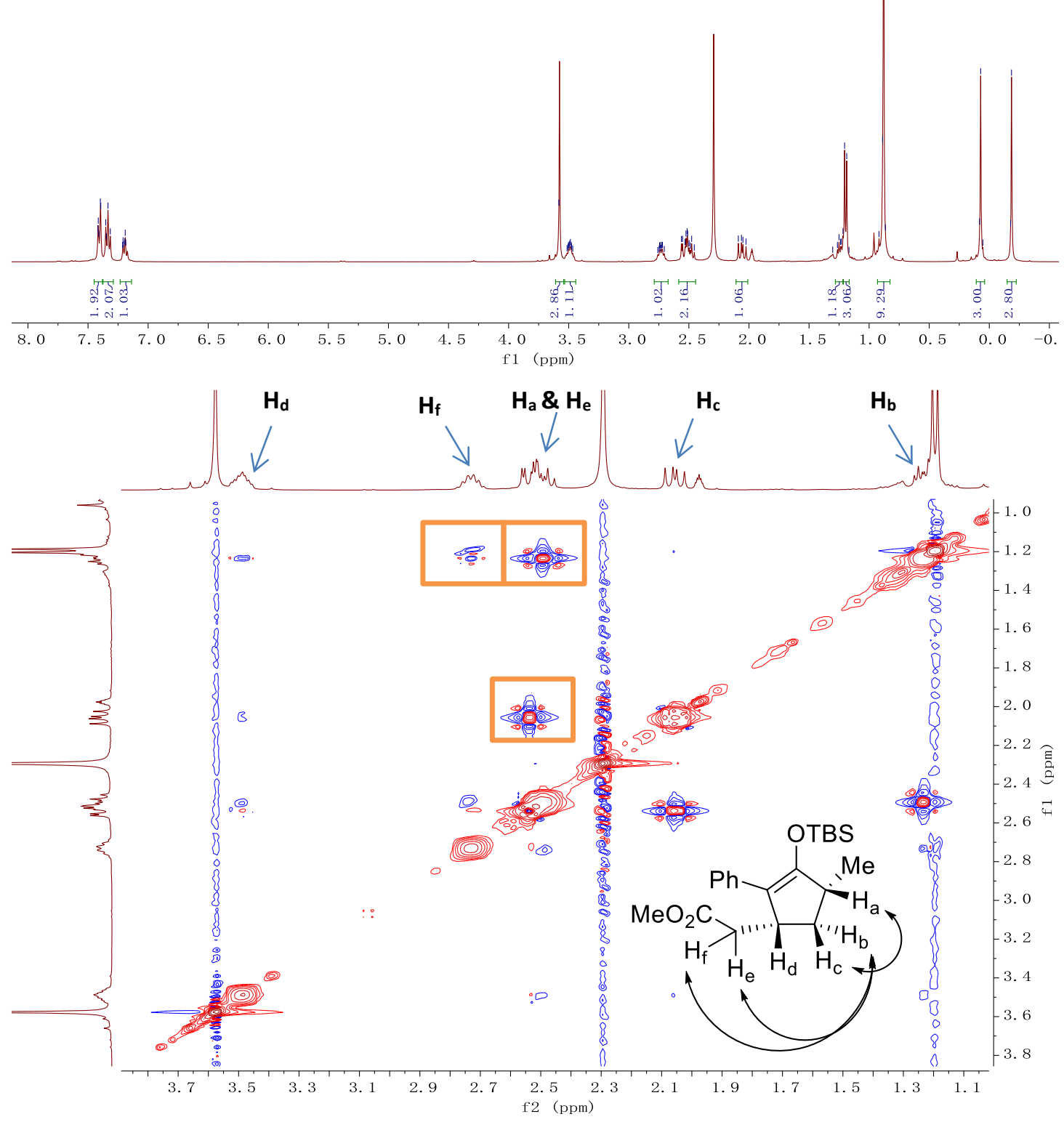


\section{(R)-6-methyl-3-phenyl-5,6-dihydropyridin-2(1H)-one (6)}

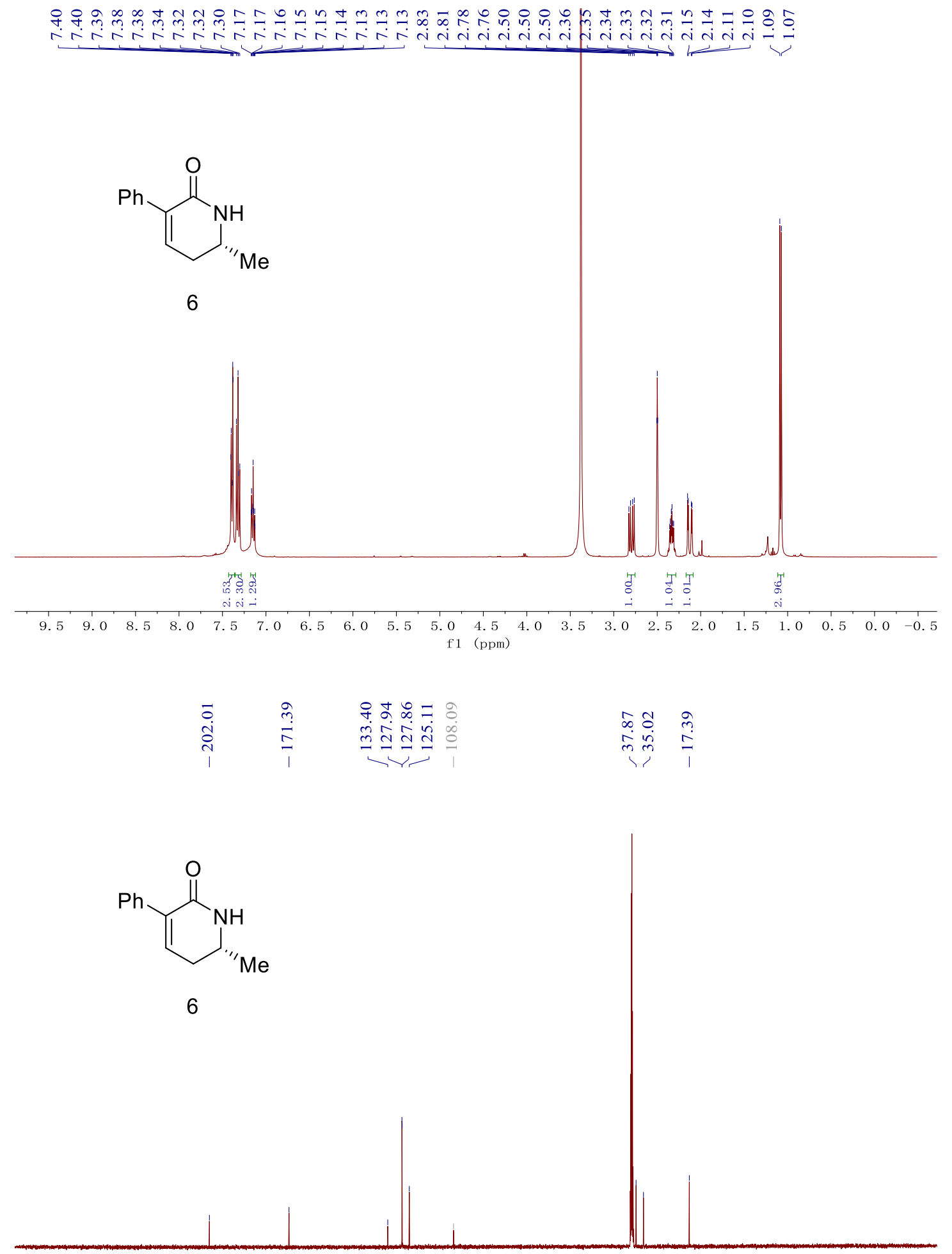

$270 \quad 250$

8

$\begin{array}{rrrrrrrrrrr}110 & 90 & 70 & 50 & 30 & 10 & -10 & -30 & -50 & -70 \\ f 1 & (\mathrm{ppm}) & & & & & & & & \end{array}$


$(1 R, 5 R)-5$-methyl-2-phenylcyclopent-2-en-1-ol (7)

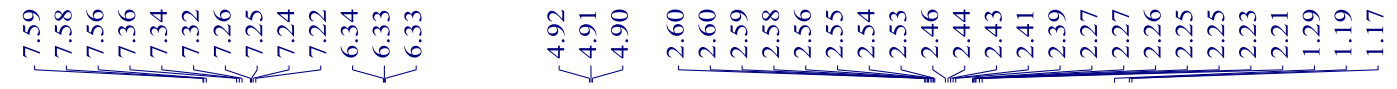

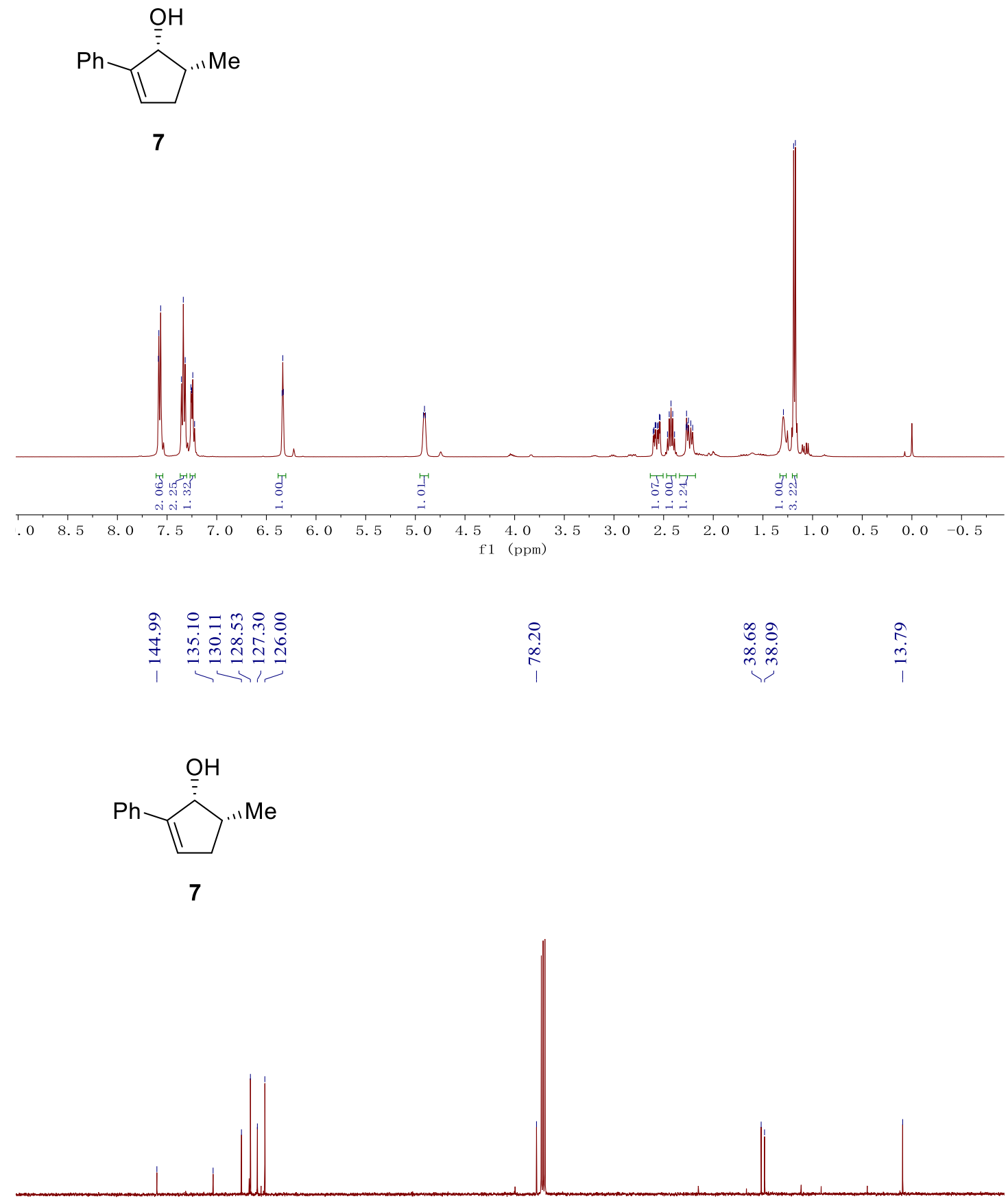

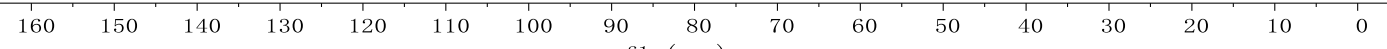

f1 (ppm) 


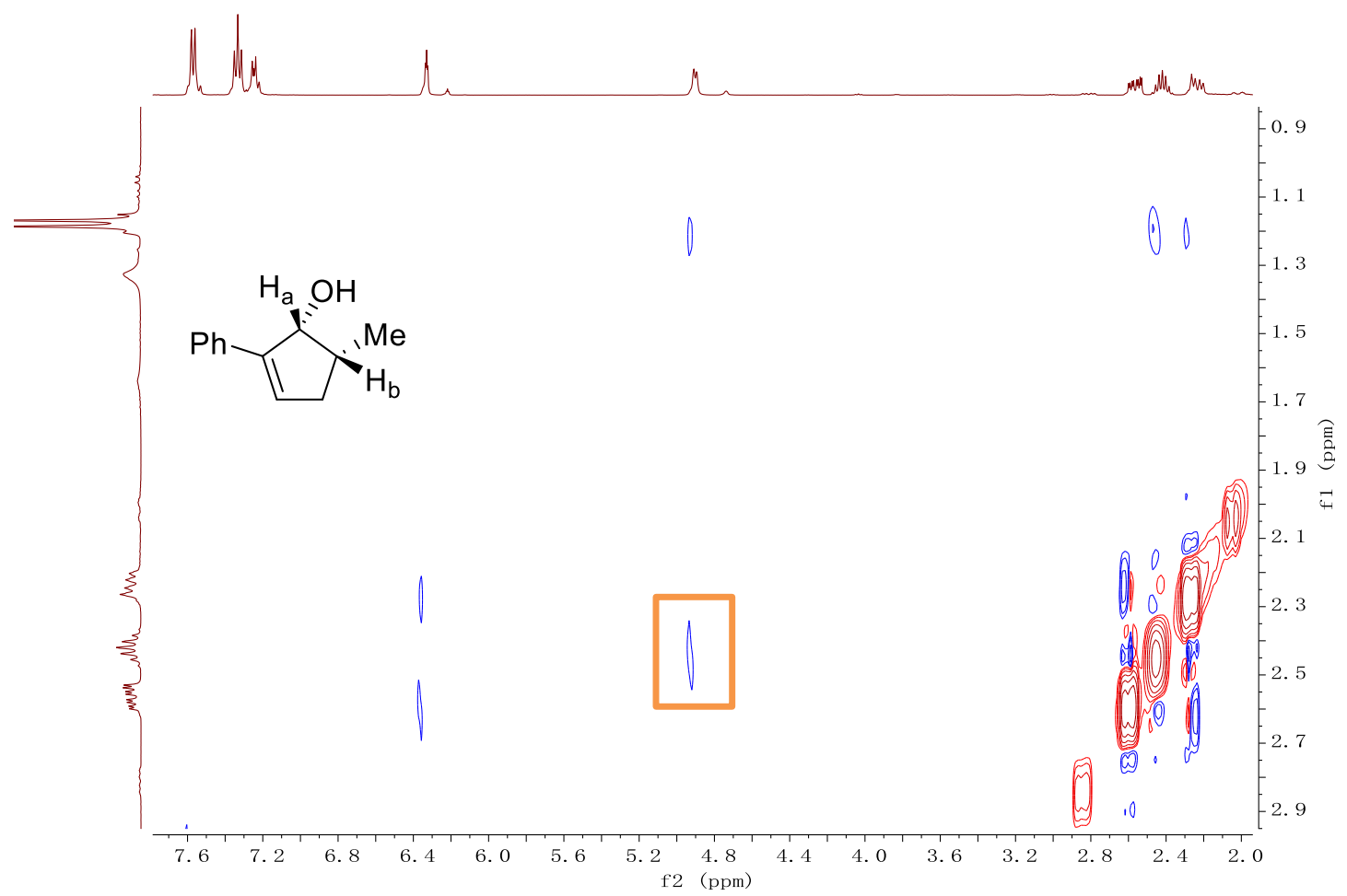



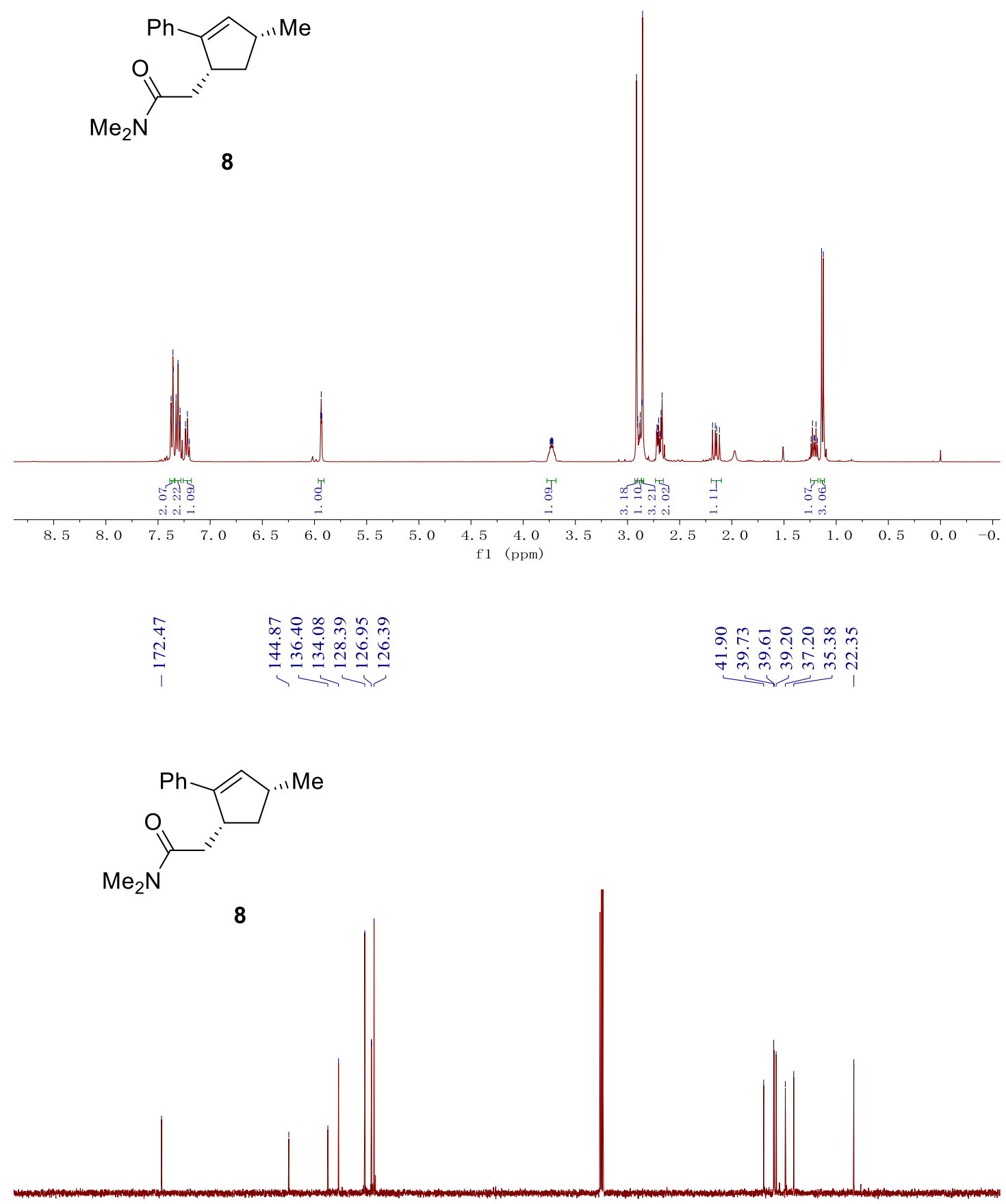

$\begin{array}{lllllllllllllllllllll}200 & 190 & 180 & 170 & 160 & 150 & 140 & 130 & 120 & 110 & 100 & 90 & 80 & 70 & 60 & 50 & 40 & 30 & 20 & 10 & 0\end{array}$ f1 (ppm) 


\section{HPLC Charts of Chiral Products}

\section{5-methyl-2-phenylcyclopent-2-en-1-one (2aa)}
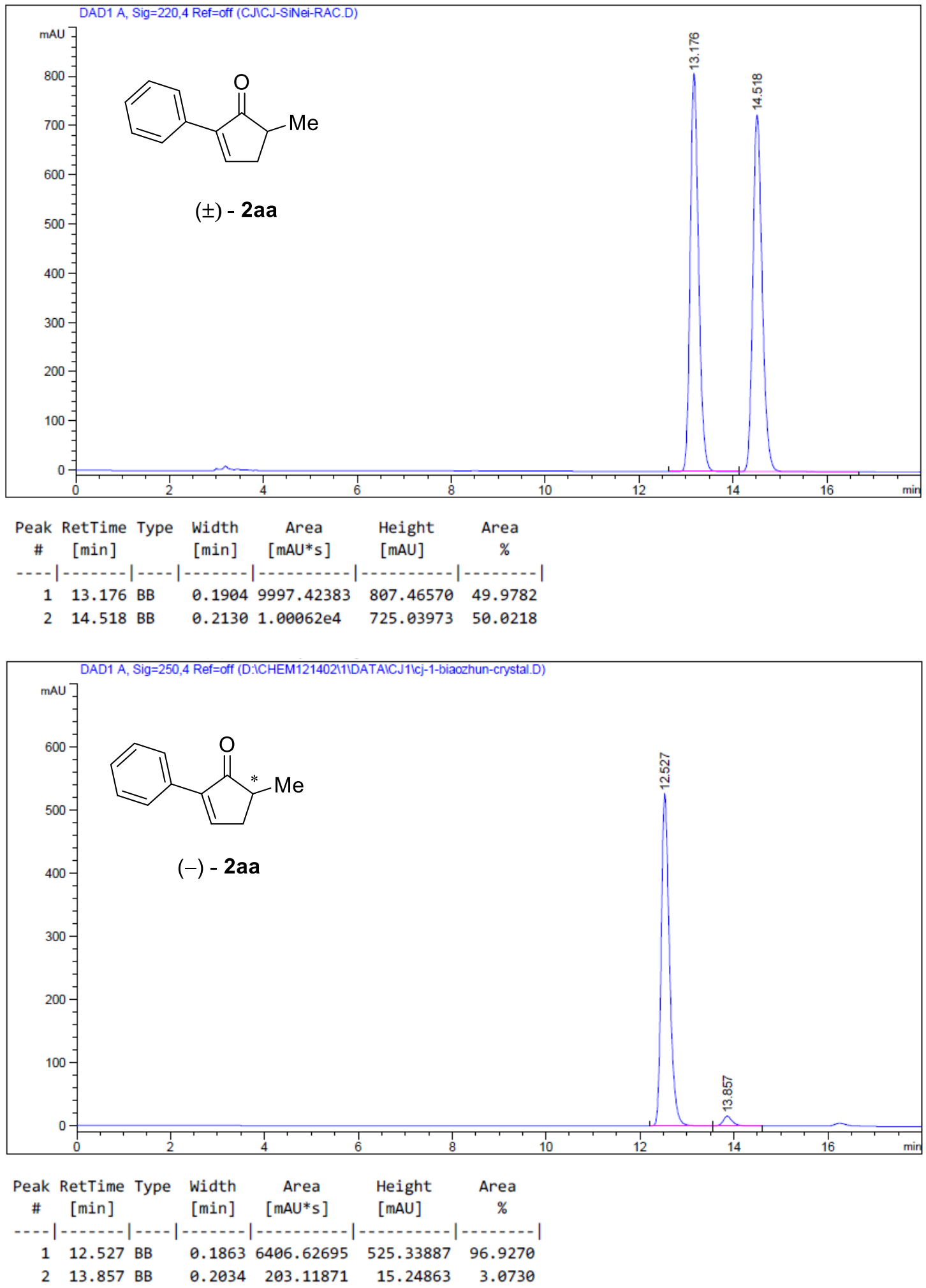
2-(4-methoxyphenyl)-5-methylcyclopent-2-en-1-one (2ab)
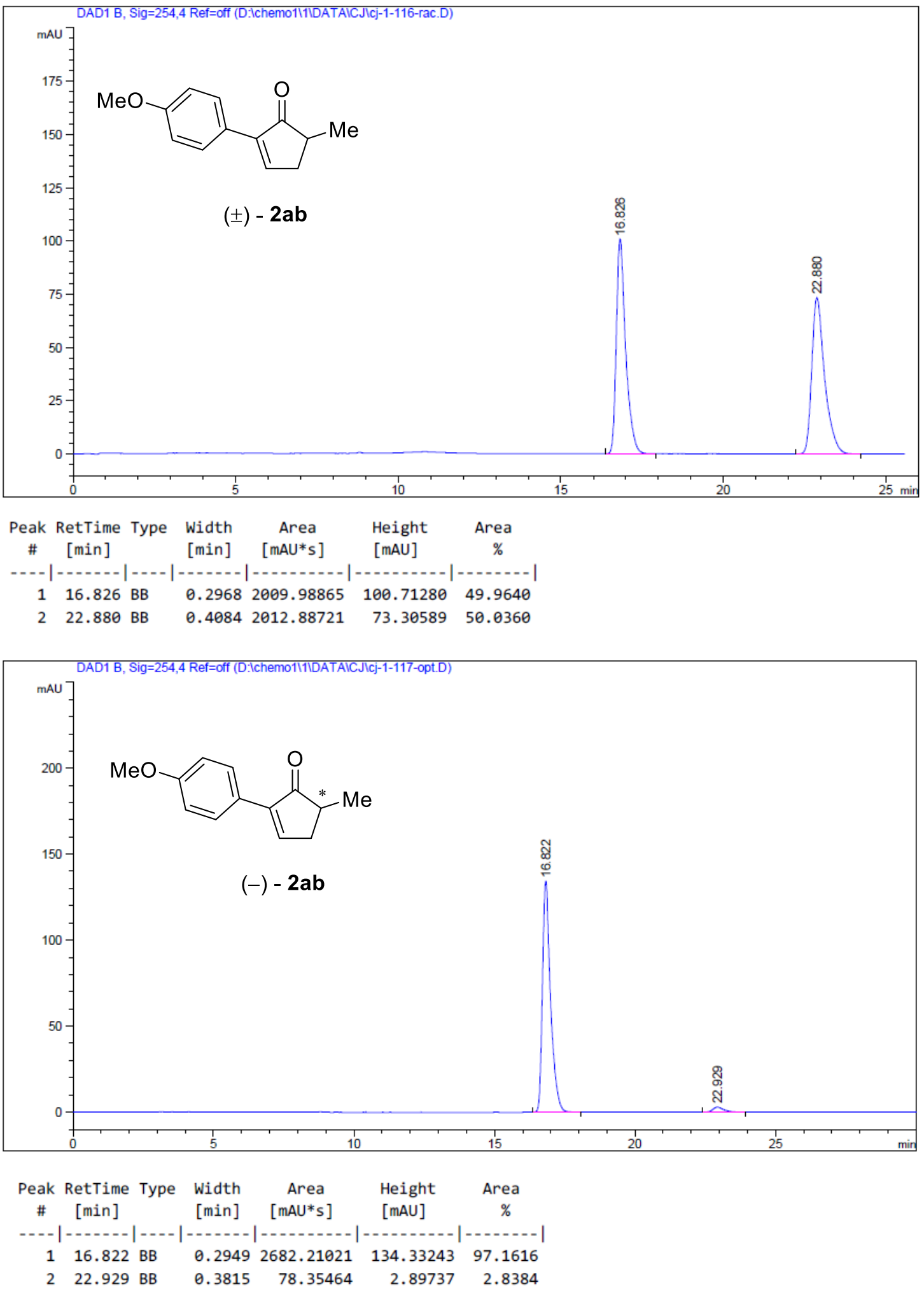


\section{5-methyl-2-(p-tolyl)cyclopent-2-en-1-one (2ac)}
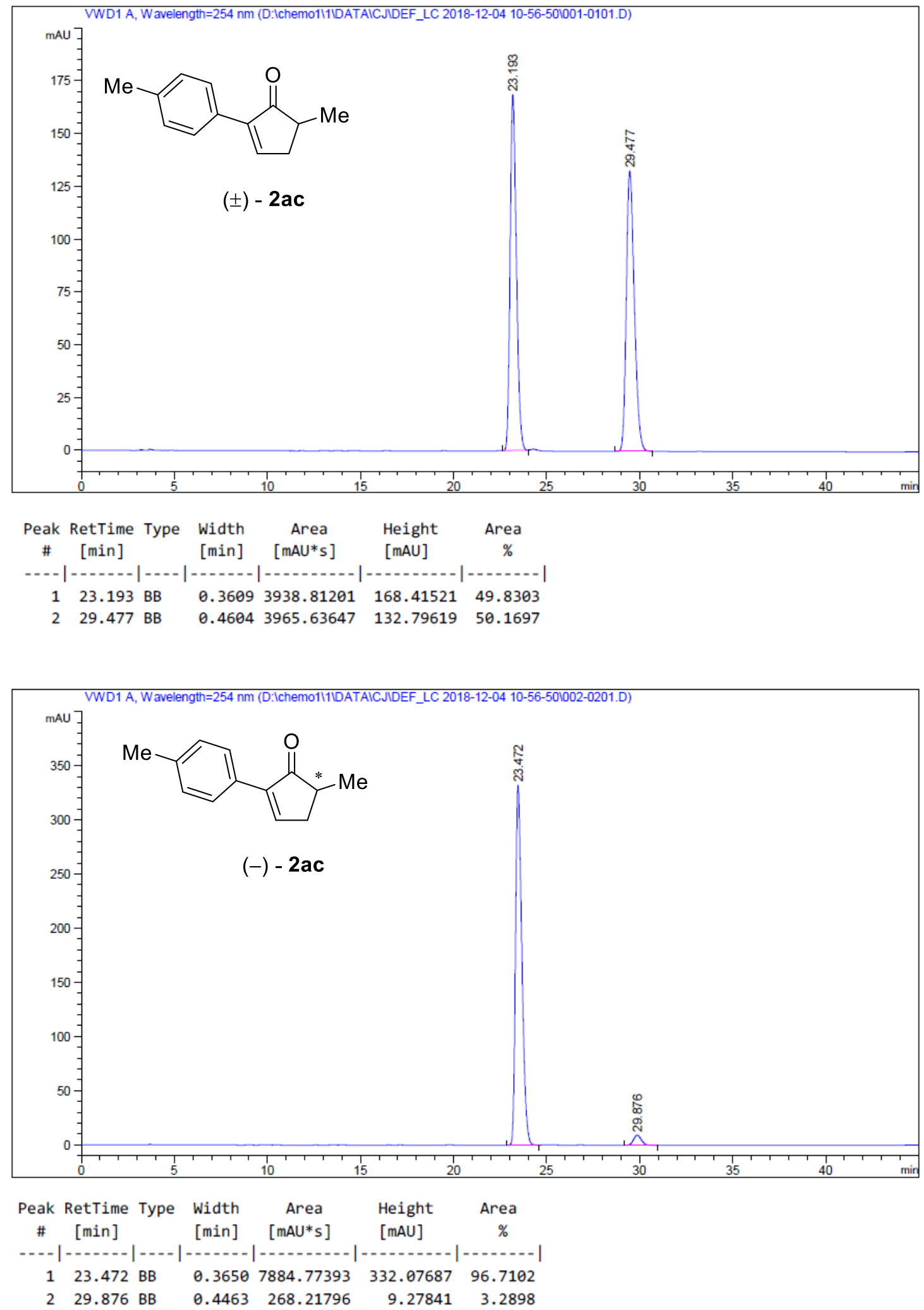
2-(4-fluorophenyl)-5-methylcyclopent-2-en-1-one (2ad)

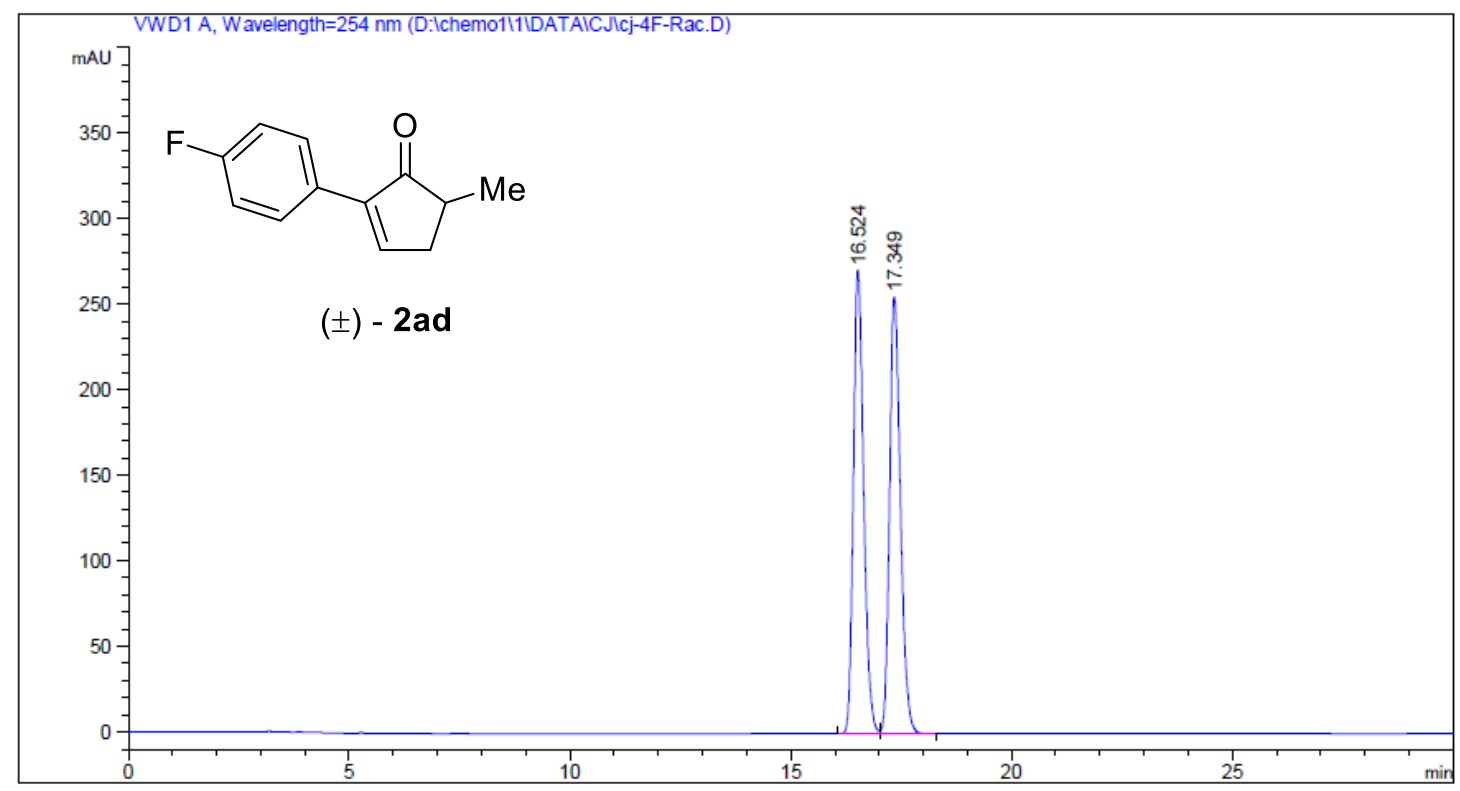

\begin{tabular}{cccccc}
$\begin{array}{c}\text { Peak RetTime Type } \\
\text { Width } \\
\text { [min] }\end{array}$ & $\begin{array}{c}\text { Area } \\
\text { [min] }\end{array}\left[\begin{array}{c}\text { Height } \\
{[\text { mAUs }]}\end{array}\right.$ & $\begin{array}{c}\text { Area } \\
\text { [mAU] }\end{array}$ & $\%$ \\
\hline 1 & 16.524 BV & 0.2484 & 4372.41406 & 270.71231 & 49.9755 \\
2 & 17.349 VB & 0.2630 & 4376.70313 & 255.32370 & 50.0245
\end{tabular}

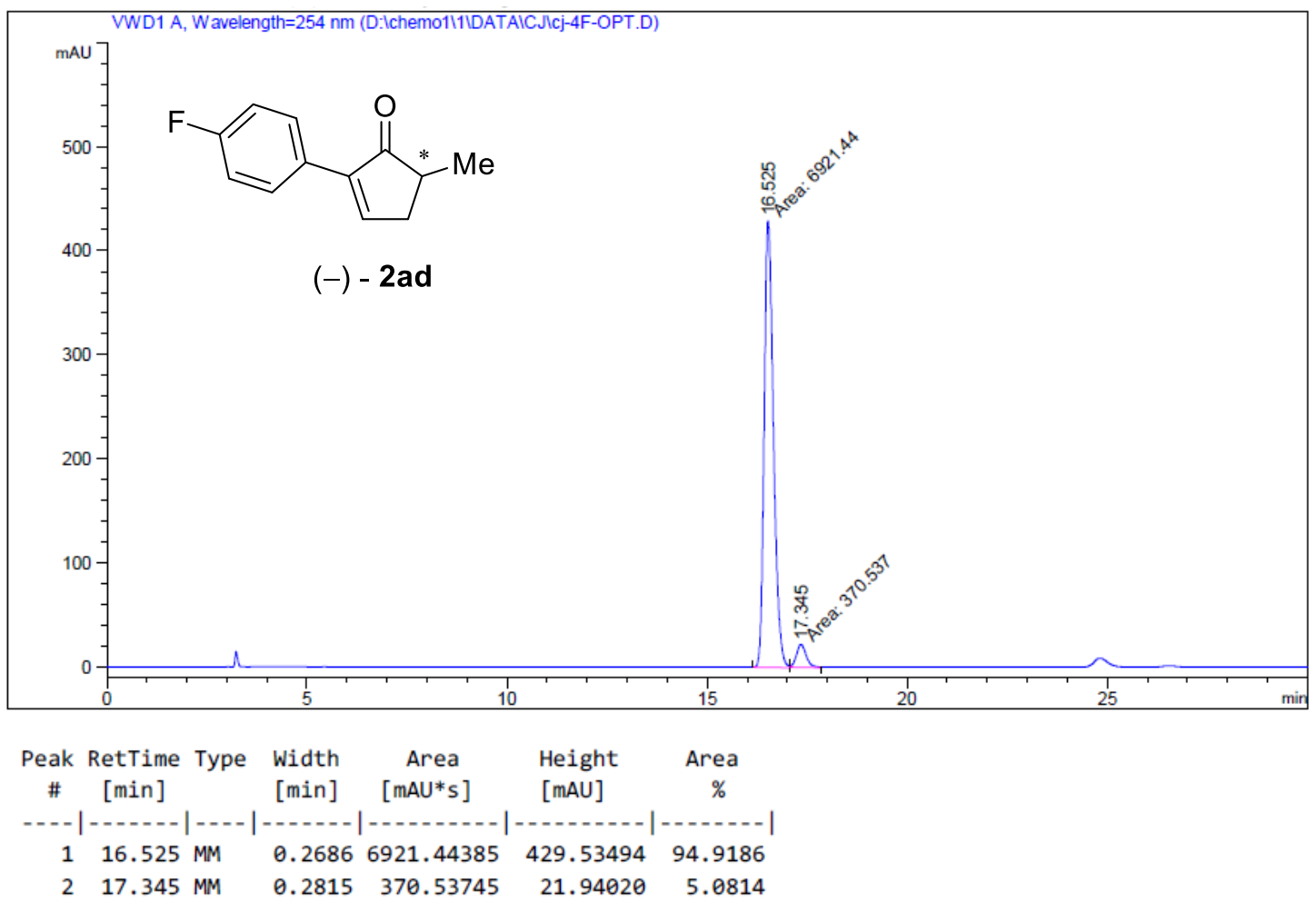


2-(4-chlorophenyl)-5-methylcyclopent-2-en-1-one (2ae)
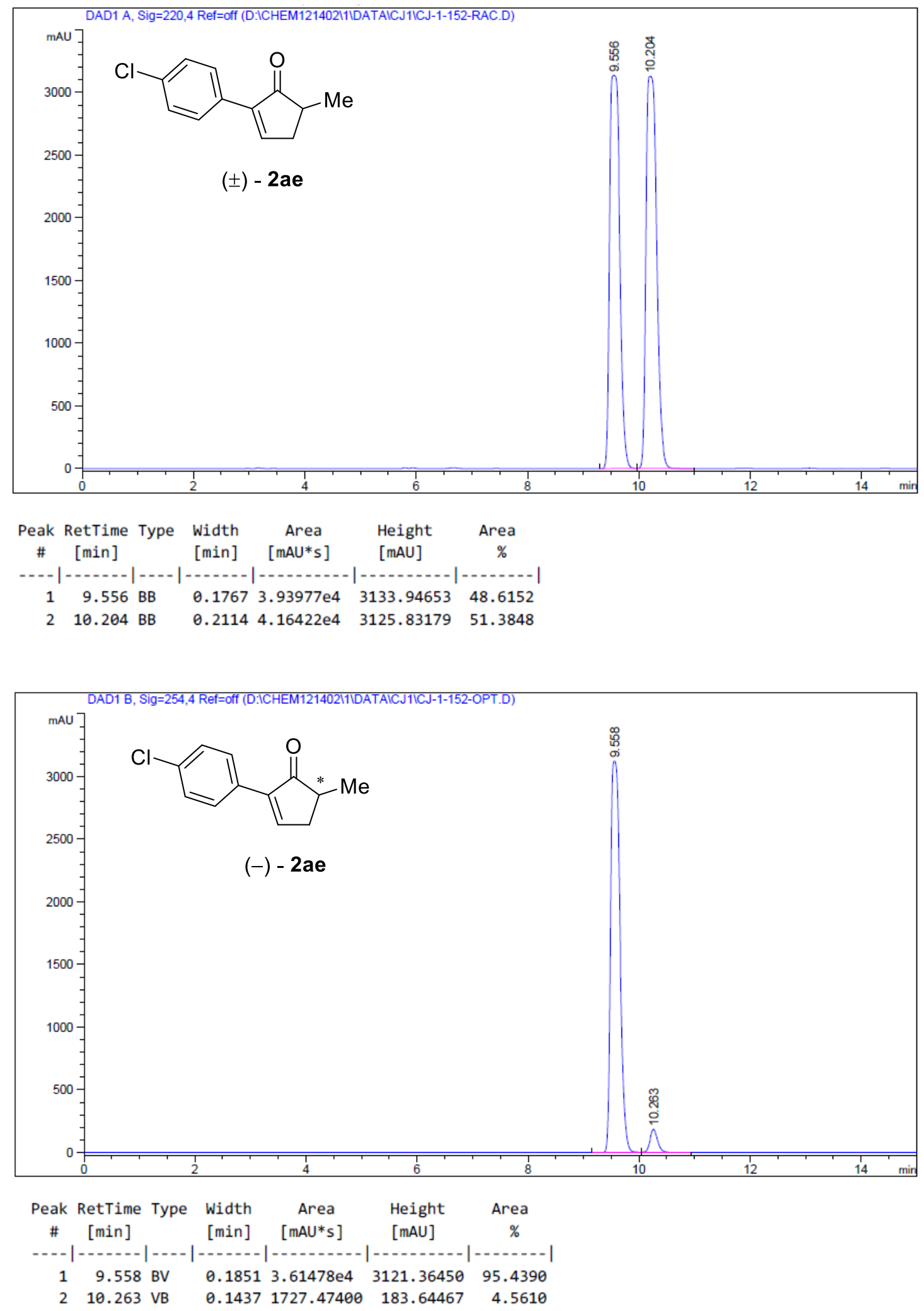
2-(4-bromophenyl)-5-methylcyclopent-2-en-1-one (2af)
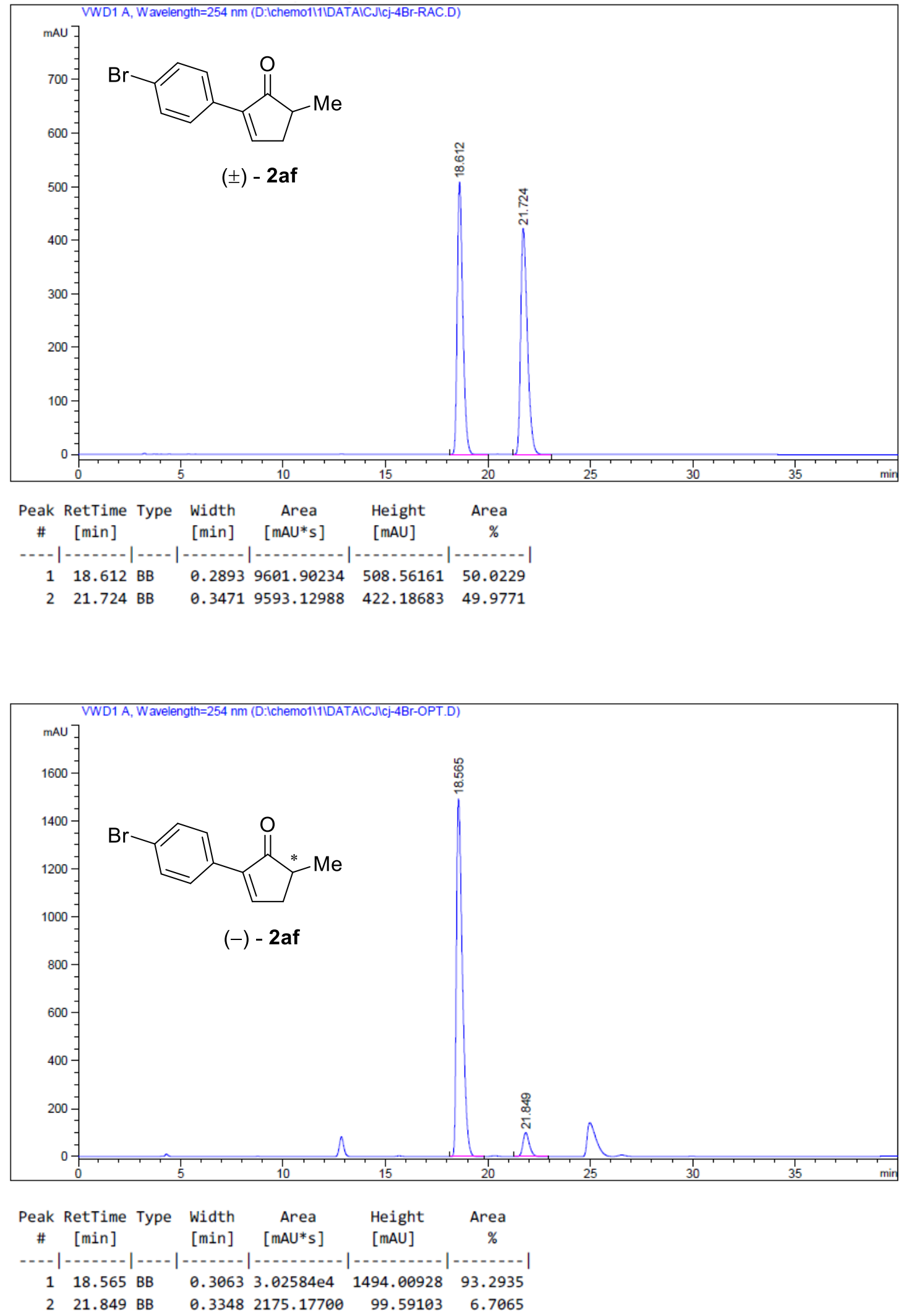
5-methyl-2-(4-(trifluoromethyl)phenyl)cyclopent-2-en-1-one (2ag)
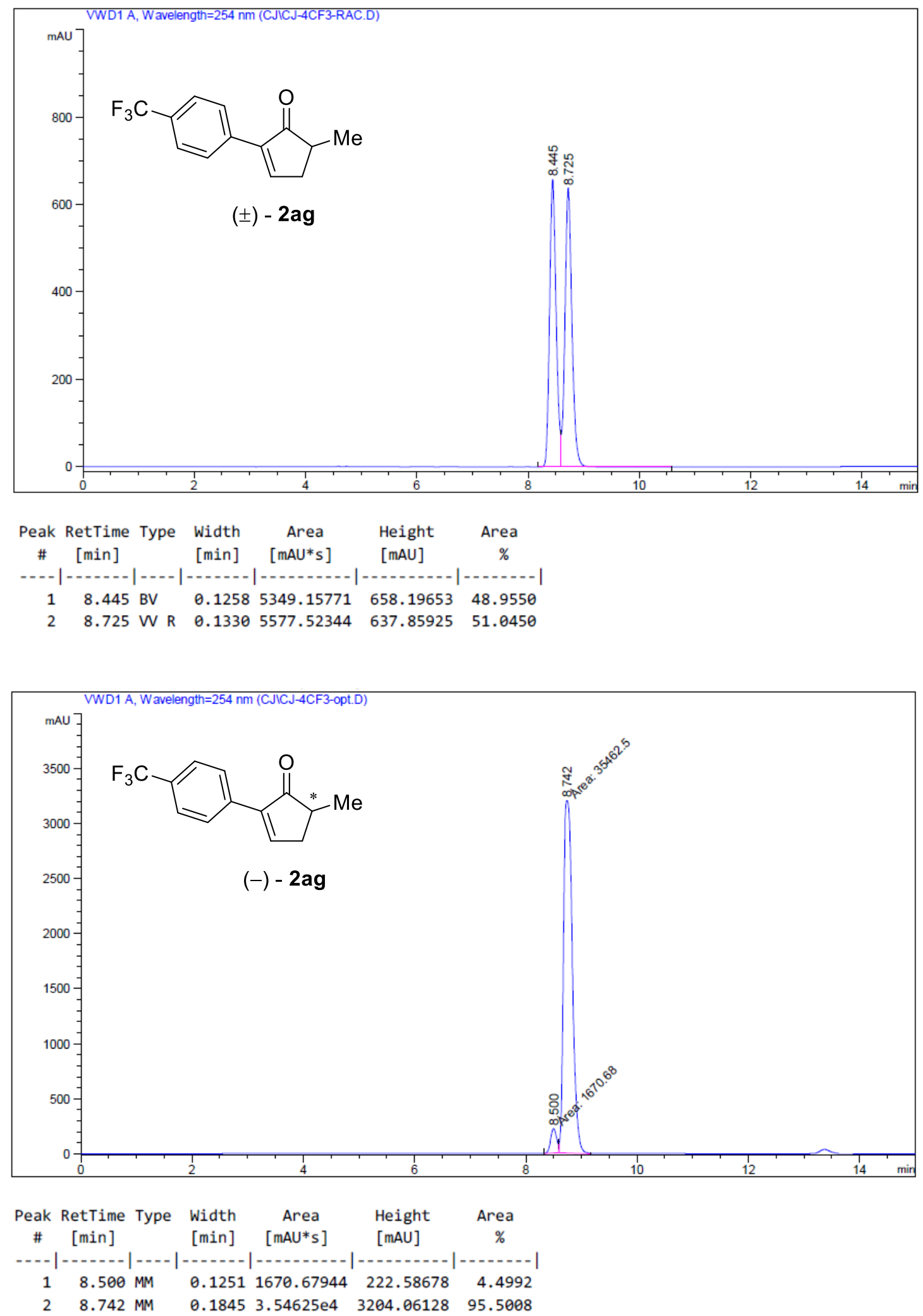


\section{5-methyl-2-(m-tolyl)cyclopent-2-en-1-one (2ah)}
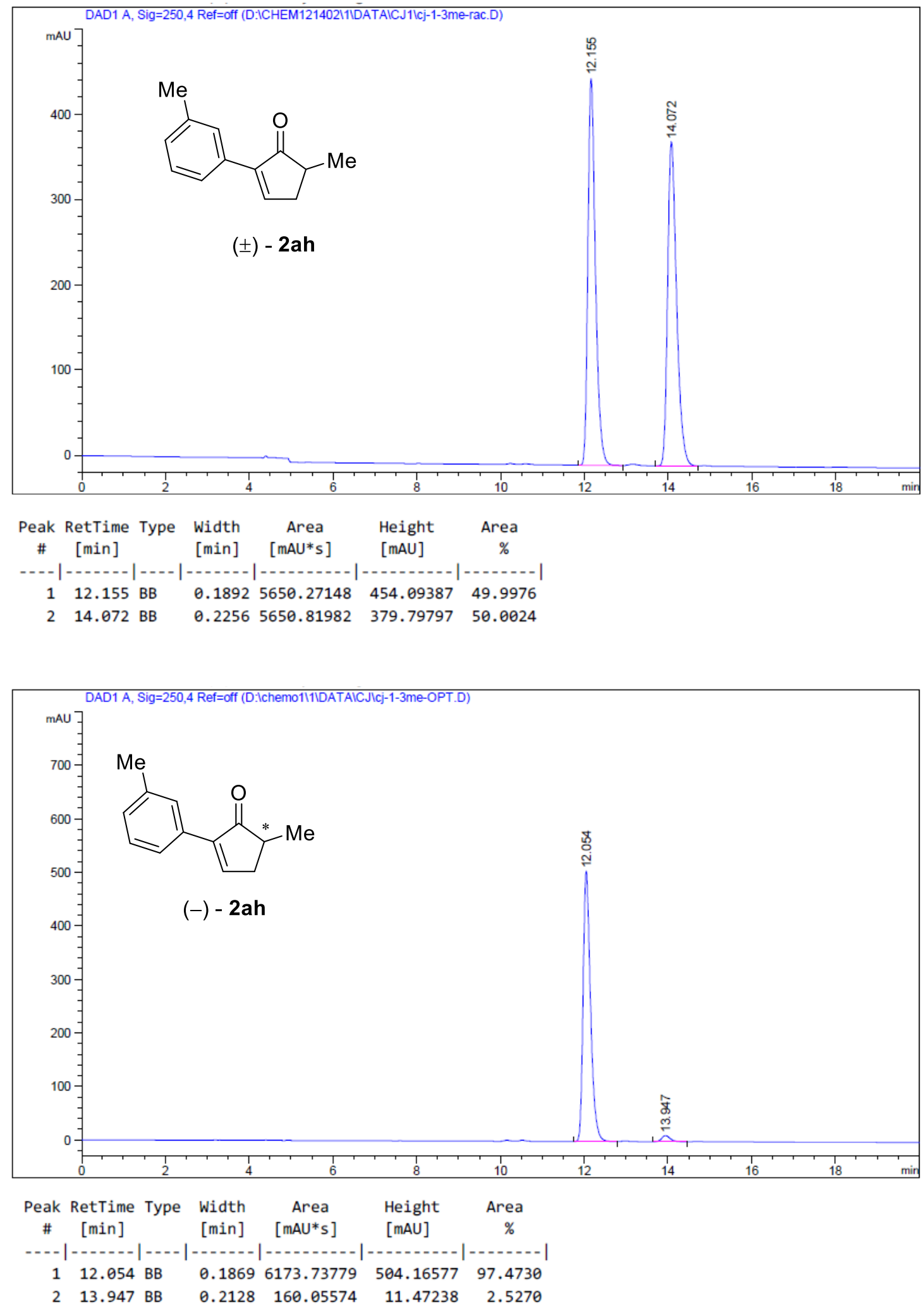
5-methyl-2-(o-tolyl)cyclopent-2-en-1-one (2ai)
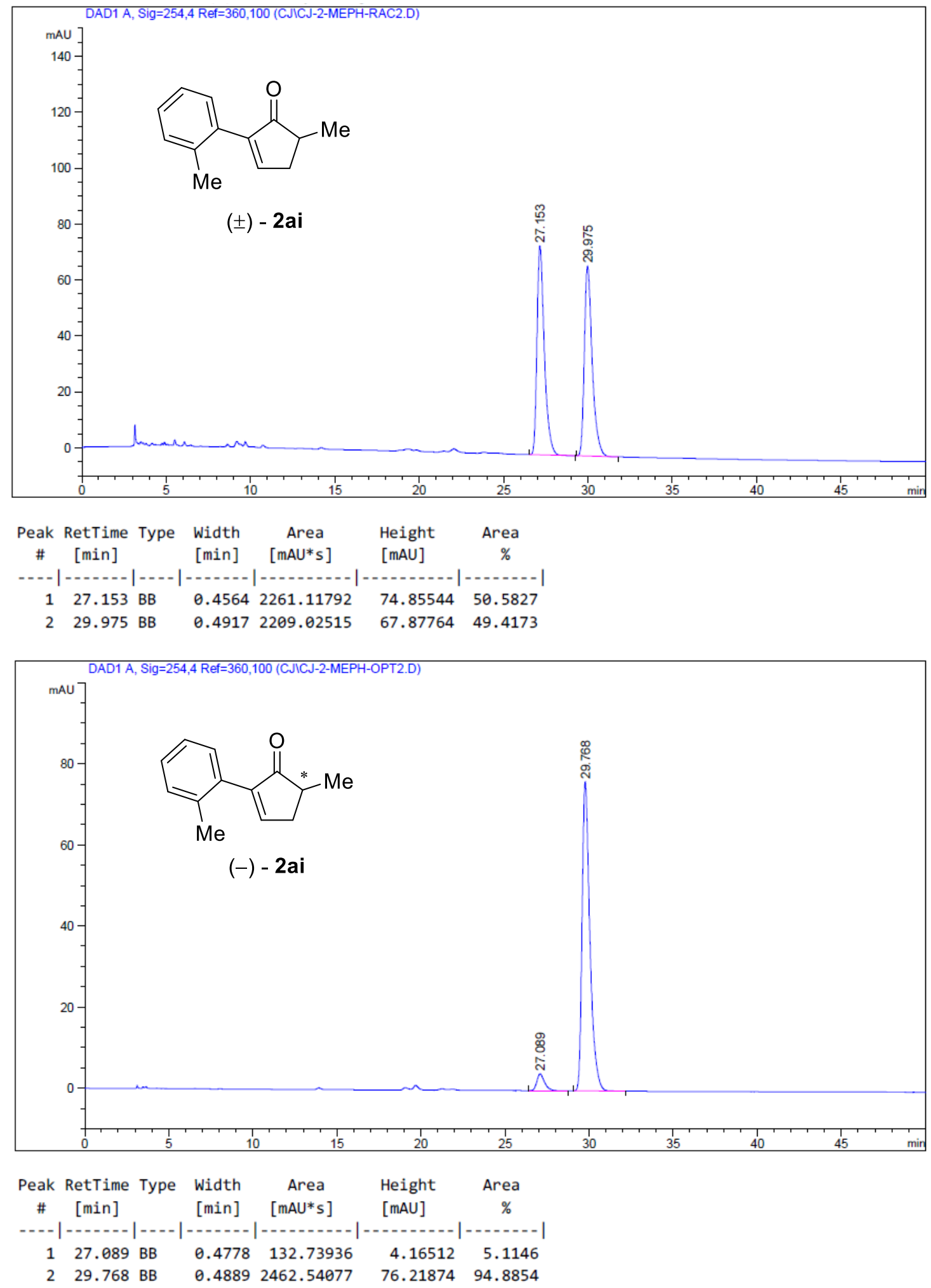
2-(benzo[d][1,3]dioxol-5-yl)-5-methylcyclopent-2-en-1-one (2aj)
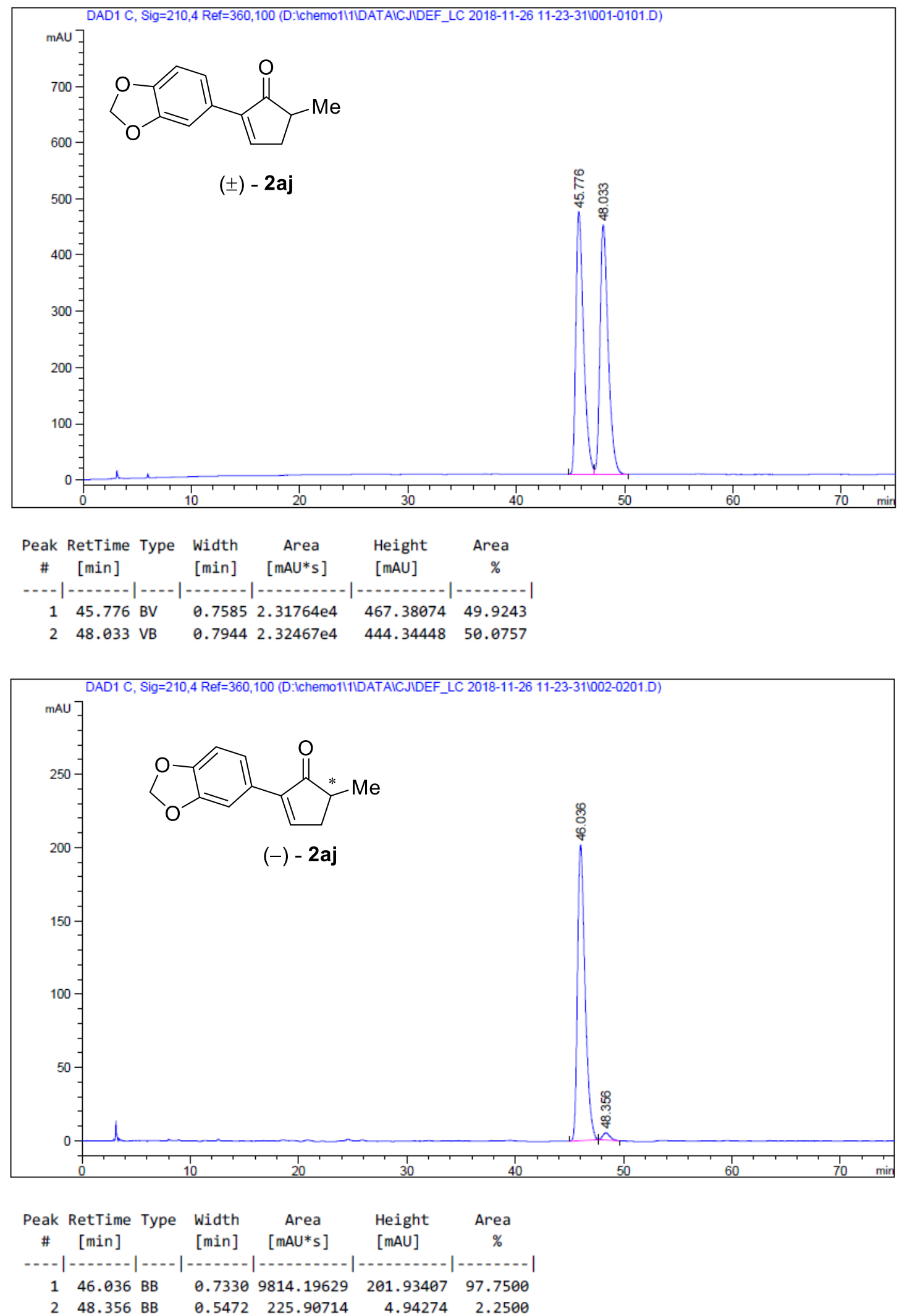
5-methyl-2-(naphthalen-2-yl)cyclopent-2-en-1-one (2ak)
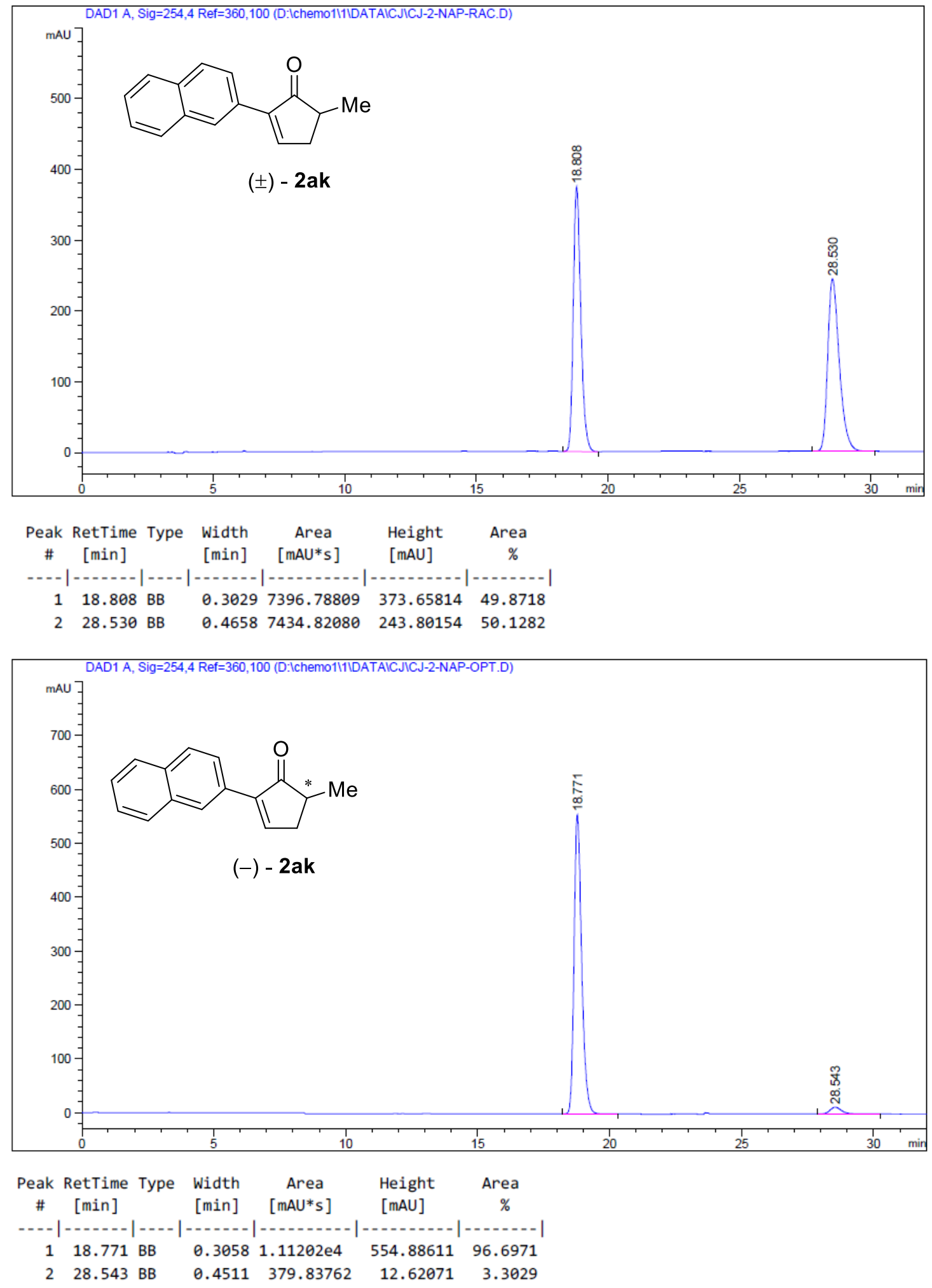
5-methyl-2-(thiophen-2-yl)cyclopent-2-en-1-one (2al)
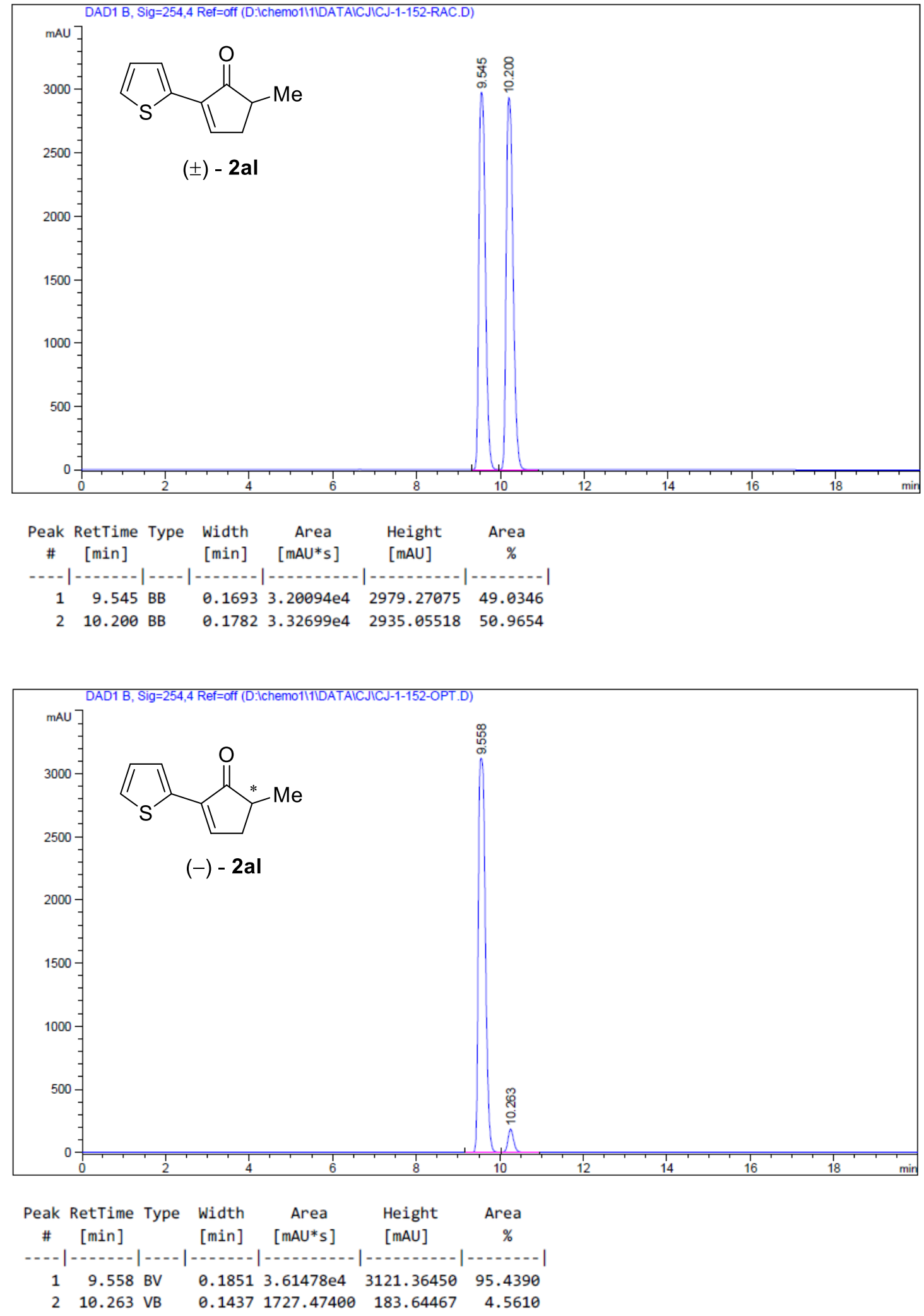
5-ethyl-2-phenylcyclopent-2-en-1-one (2ba)
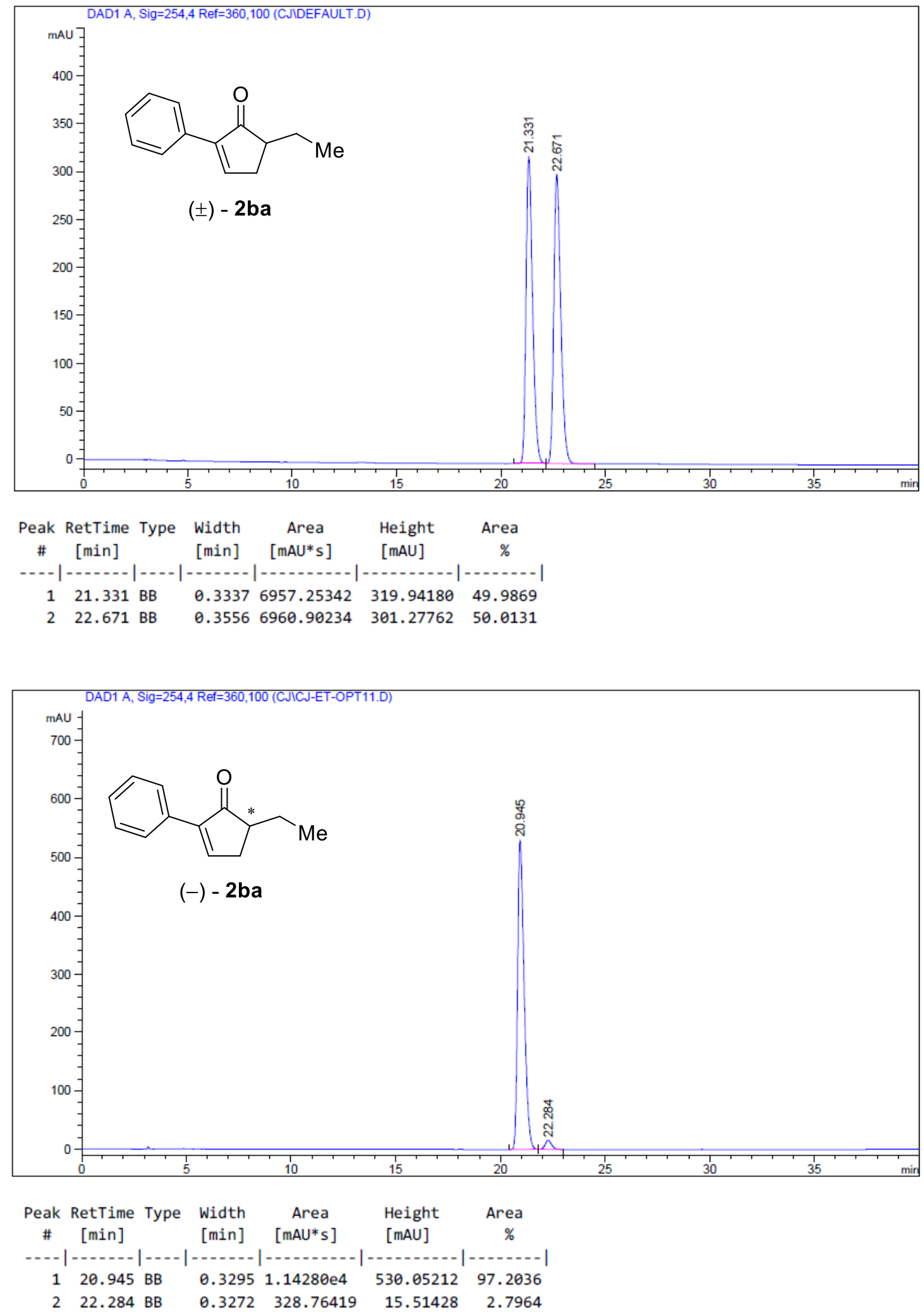
2-phenyl-5-propylcyclopent-2-en-1-one (2bb)
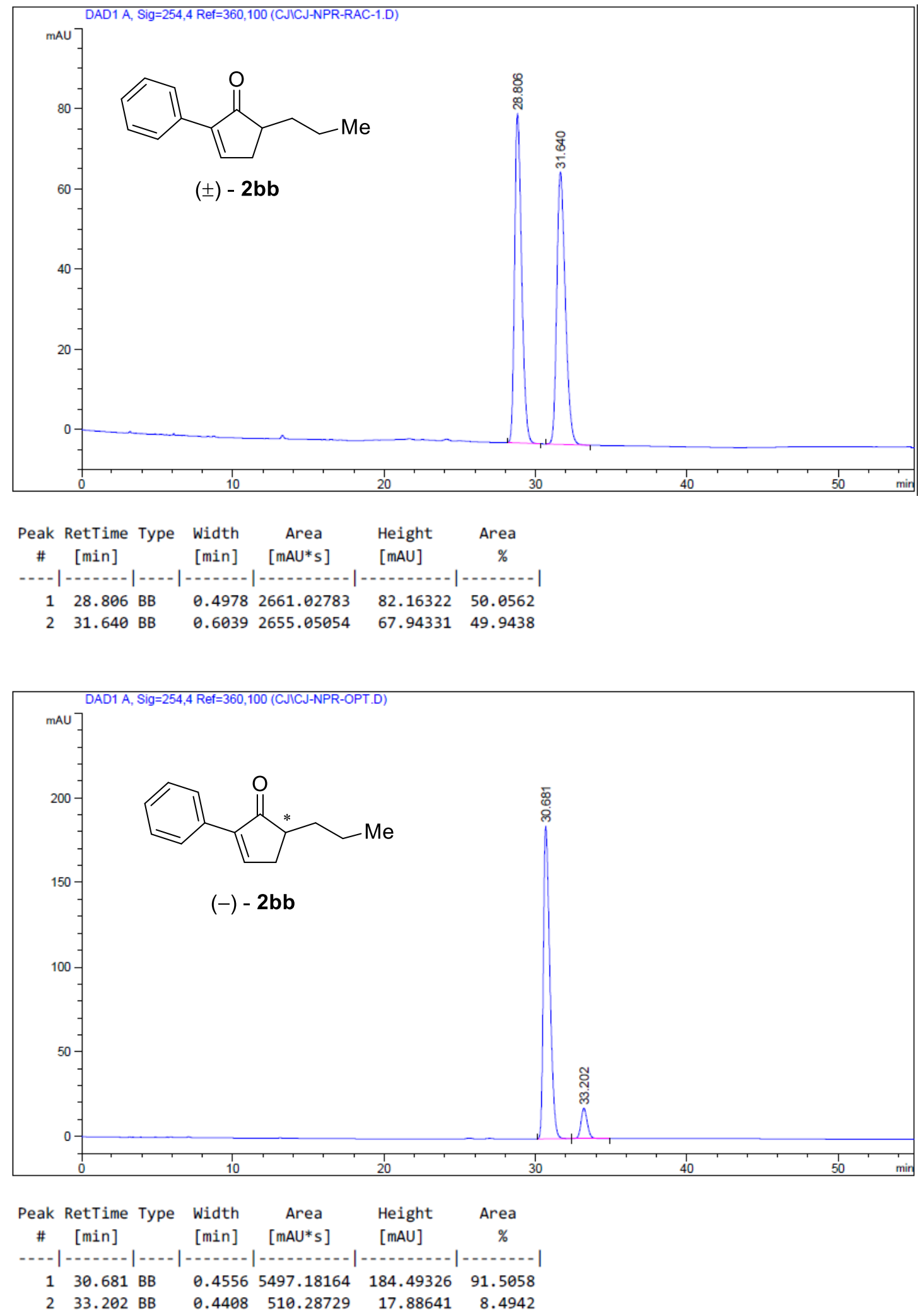


\section{5-hexyl-2-phenylcyclopent-2-en-1-one (2bc)}
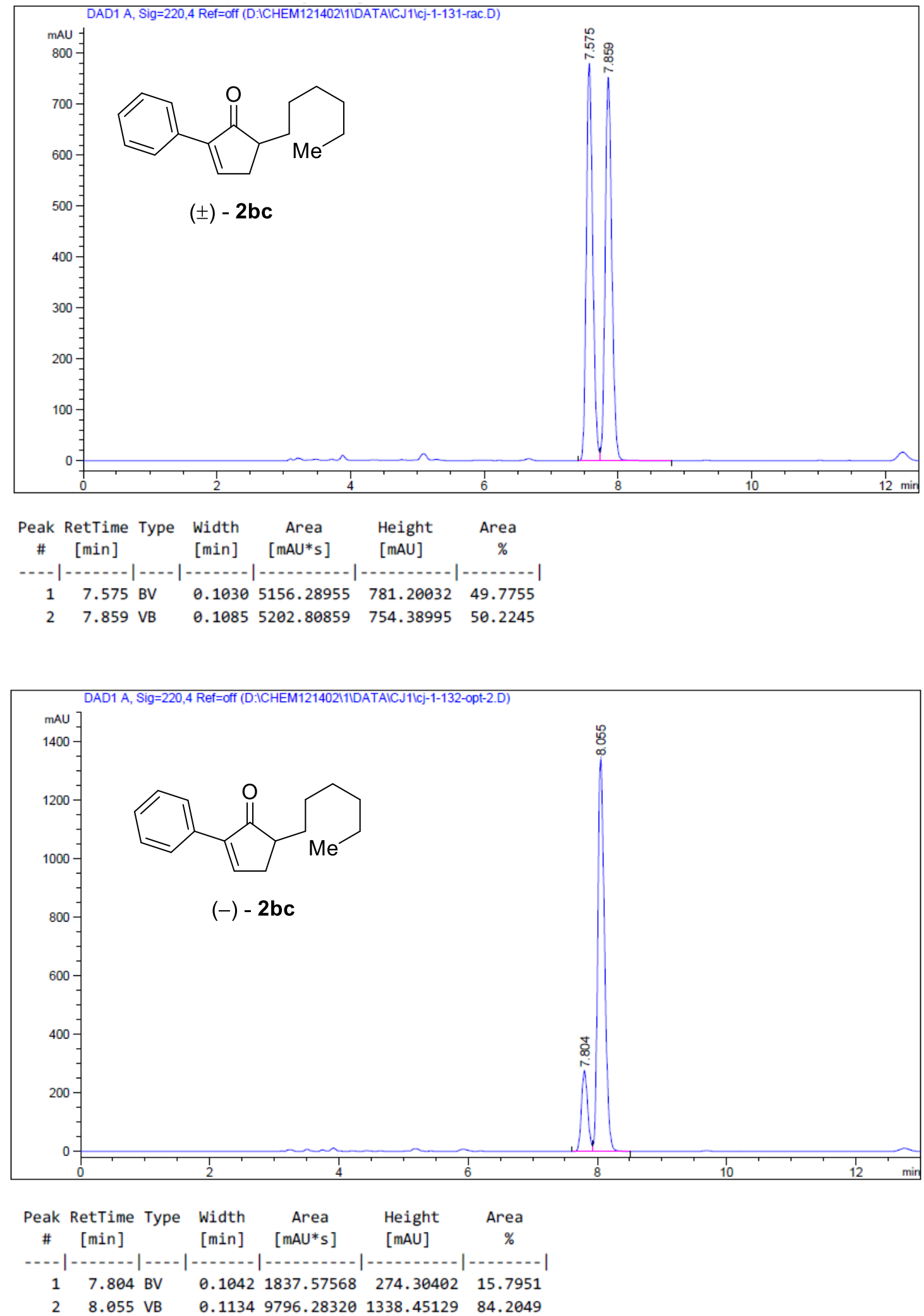
5-isopropyl-2-phenylcyclopent-2-en-1-one (2bd)
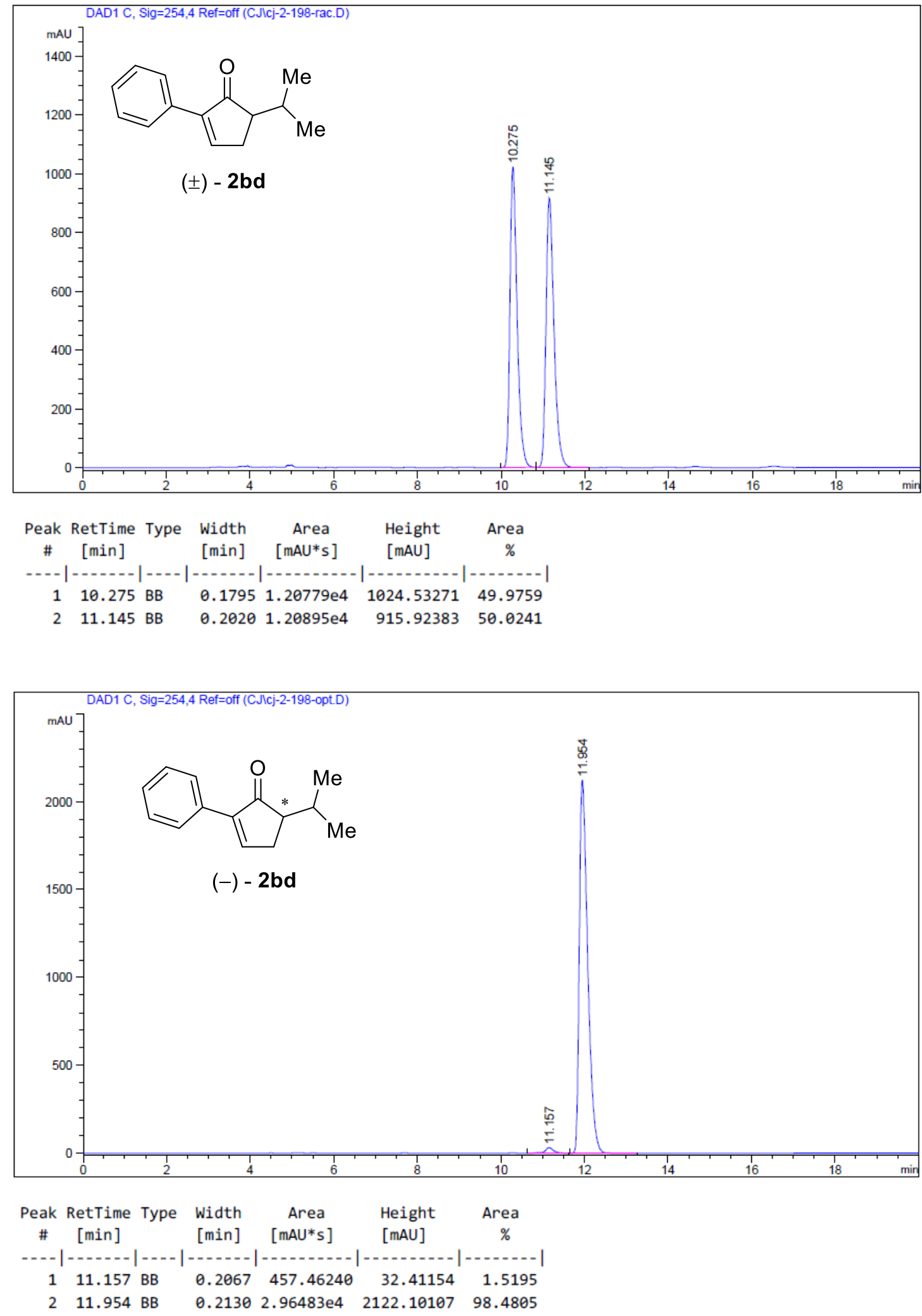
5-cyclopropyl-2-phenylcyclopent-2-en-1-one (2be)
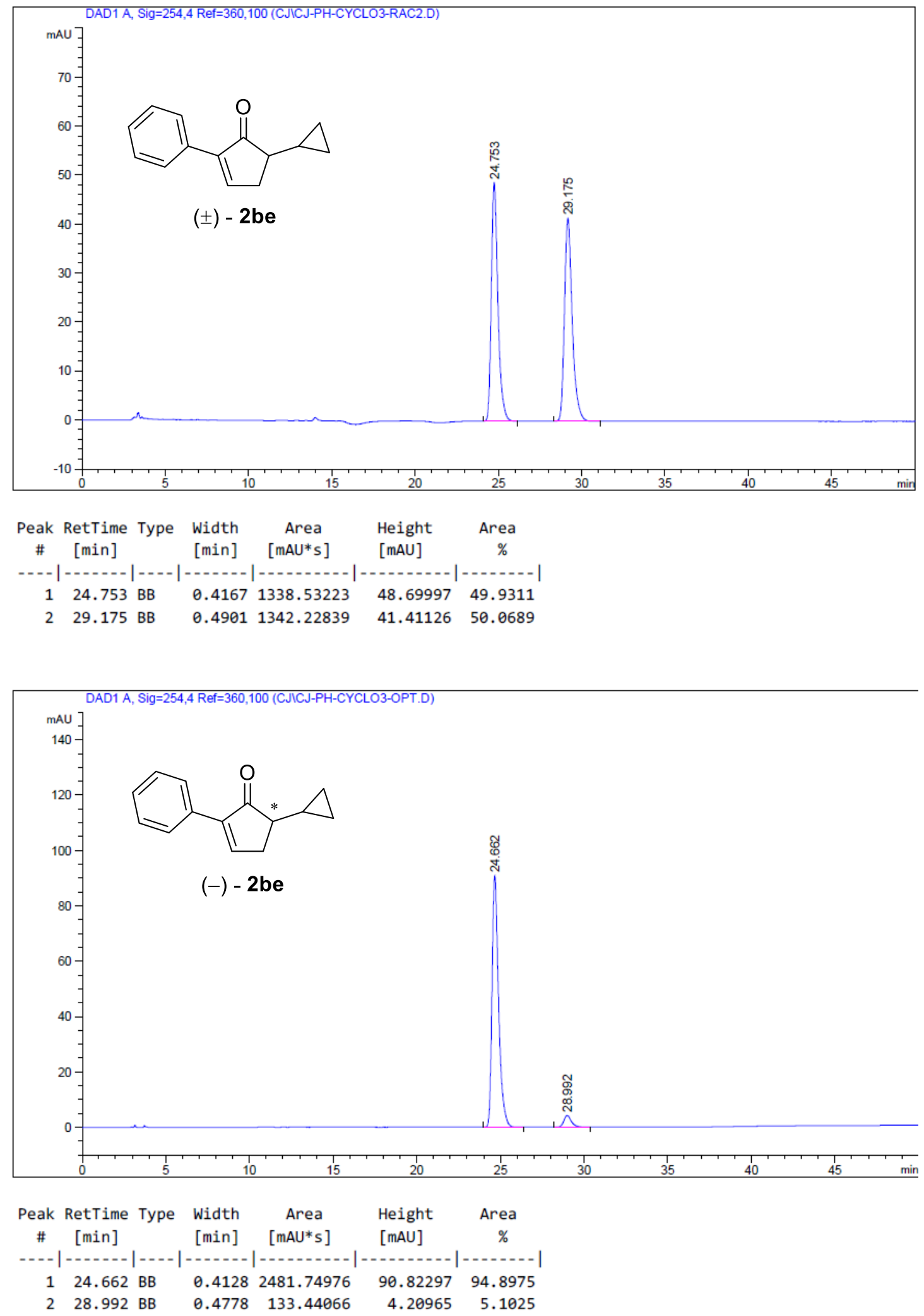
5-(but-3-en-1-yl)-2-phenylcyclopent-2-en-1-one (2bf)
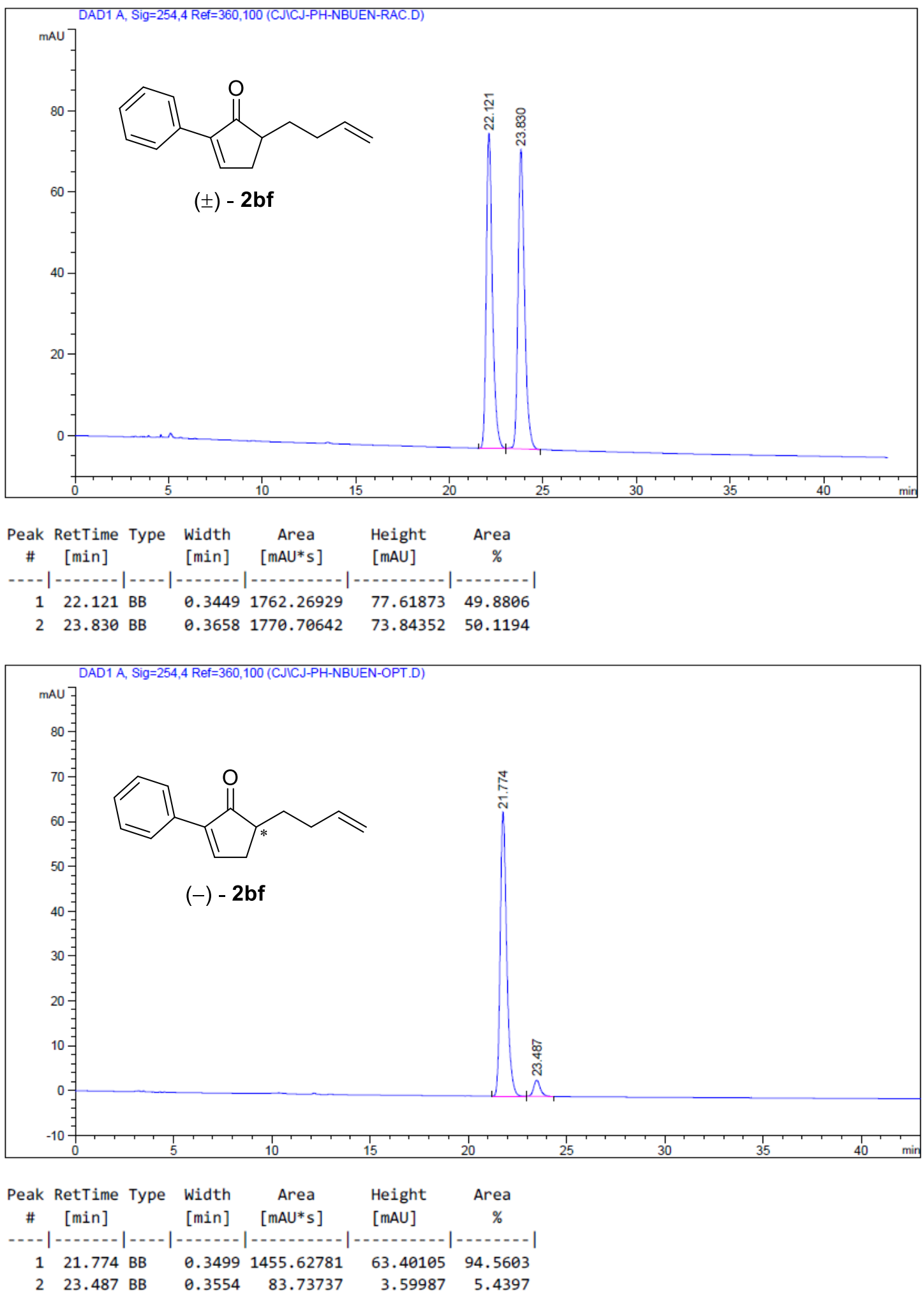


\section{5-(2-chloroethyl)-2-phenylcyclopent-2-en-1-one (2bg)}
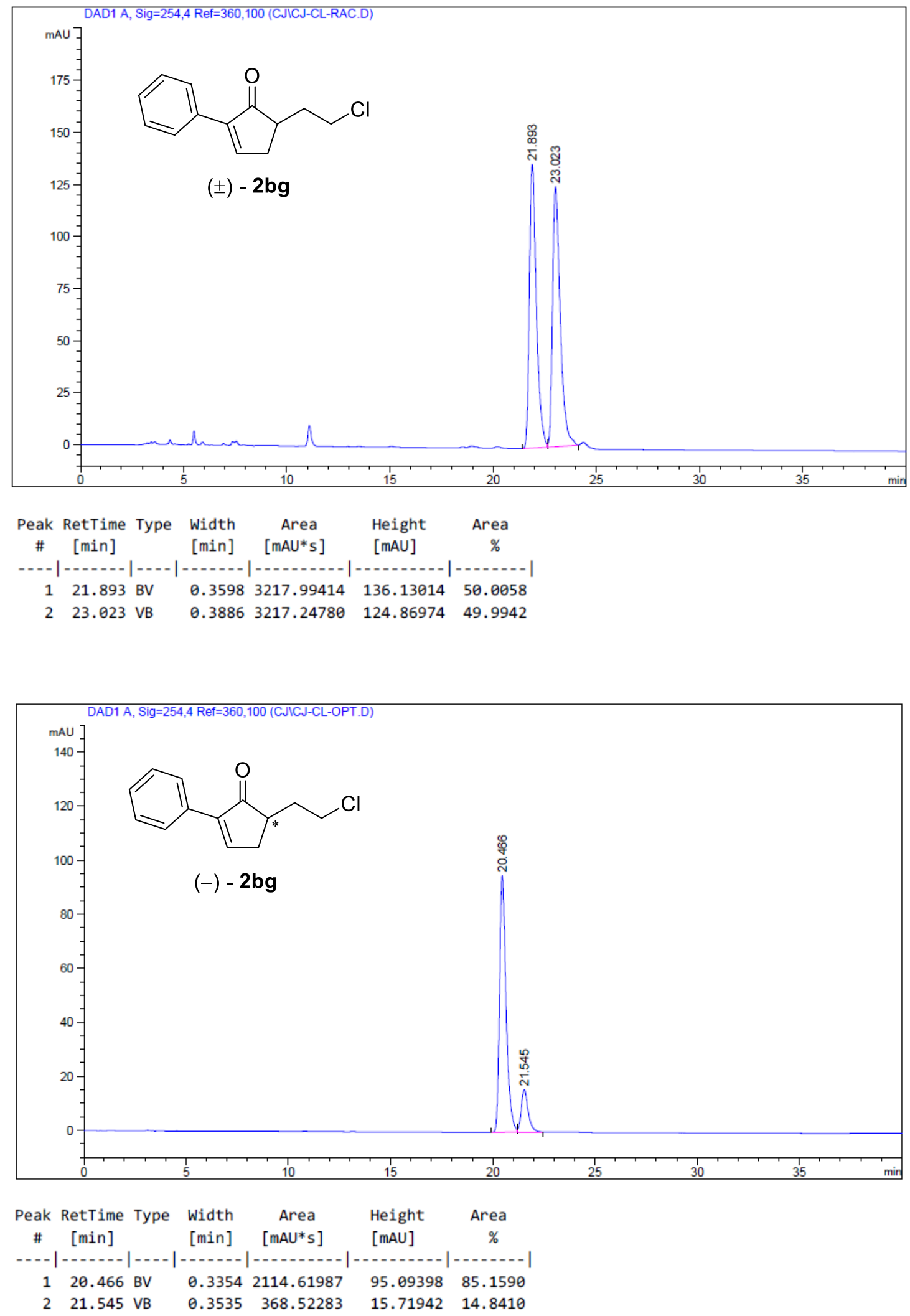
5-(2-methoxyethyl)-2-phenylcyclopent-2-en-1-one (2bh)
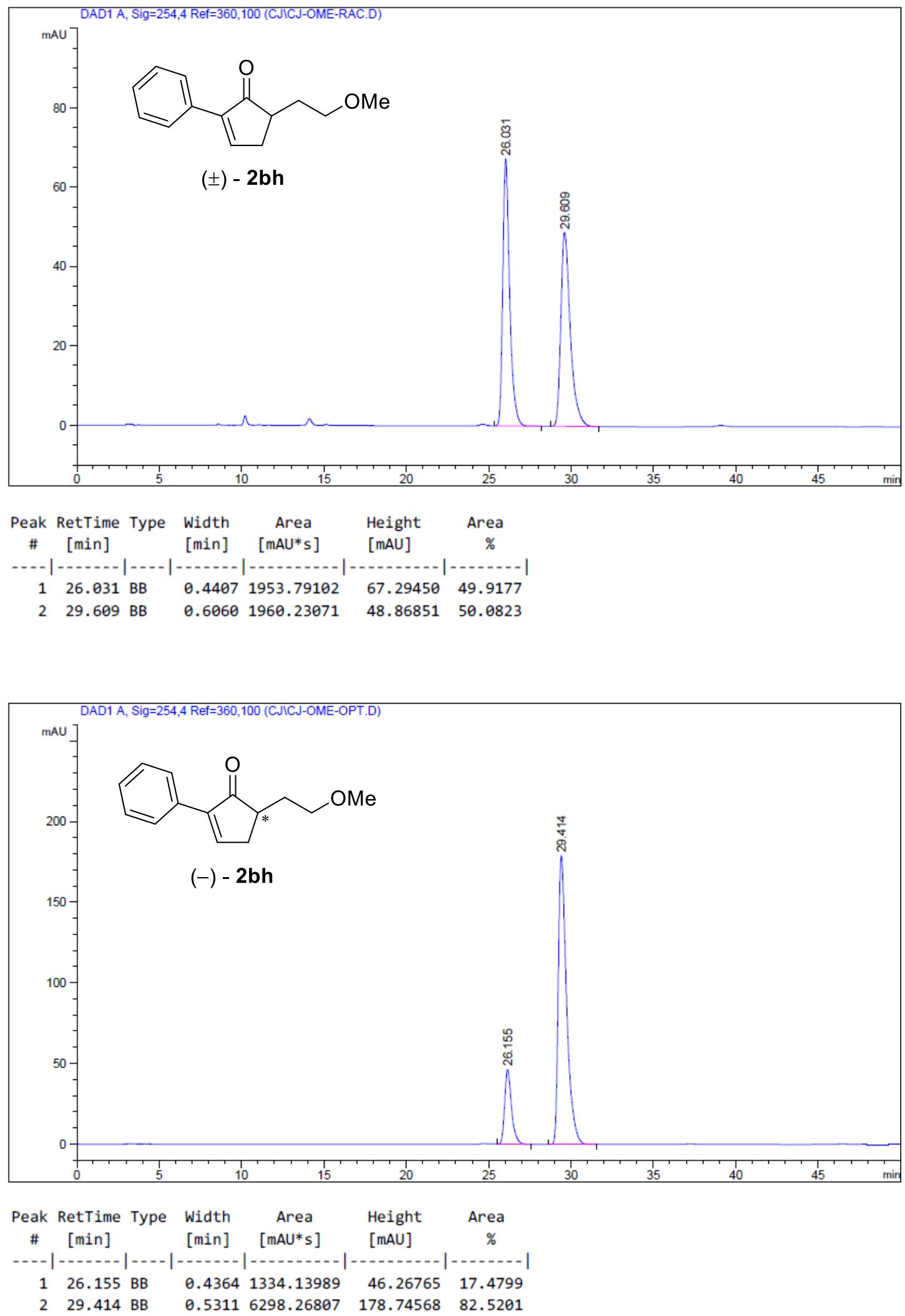


\section{5-benzyl-2-phenylcyclopent-2-en-1-one (2bi)}
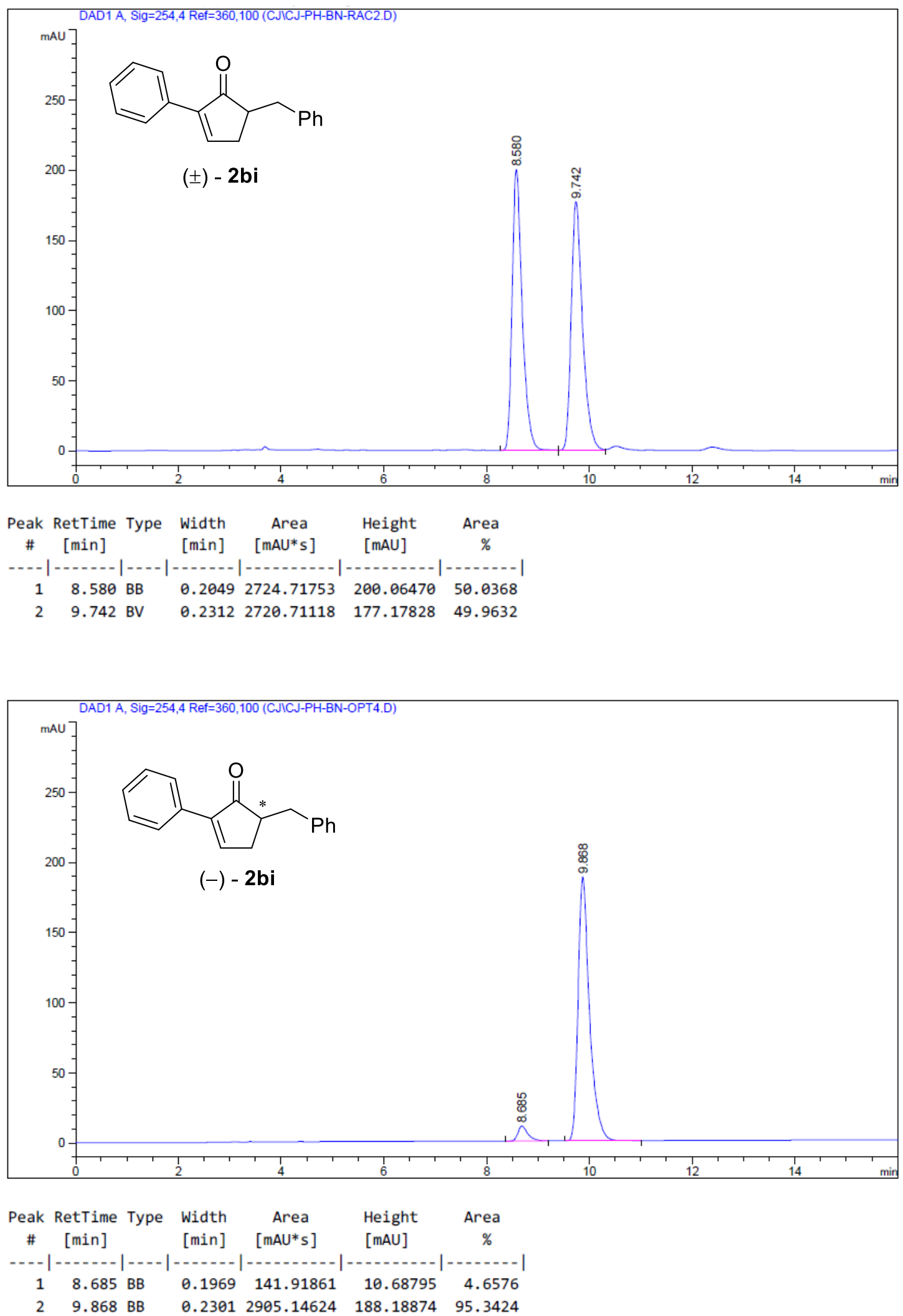
2,5-diphenylcyclopent-2-en-1-one (2ca)
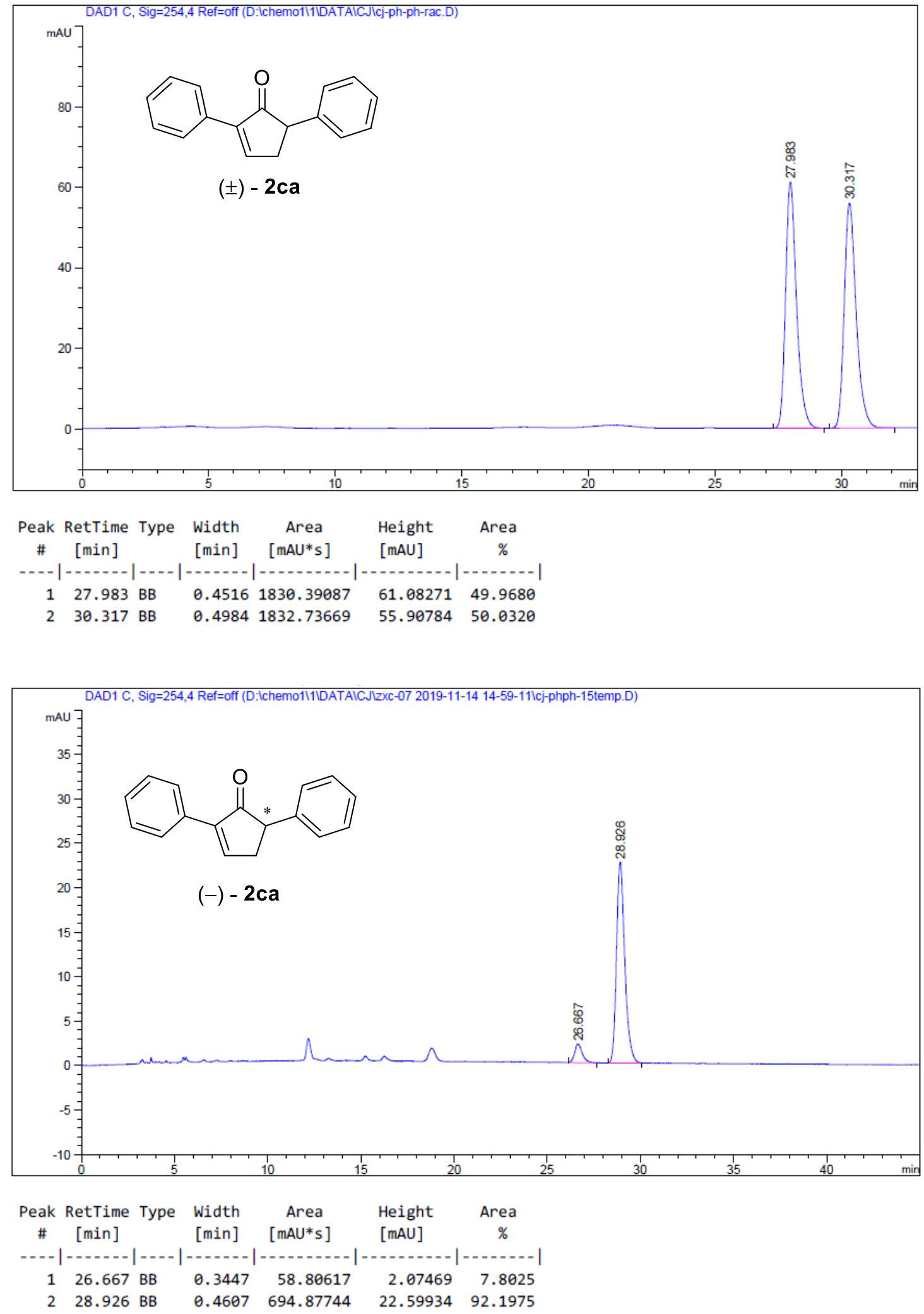
2-phenyl-5-(o-tolyl)cyclopent-2-en-1-one (2cb)
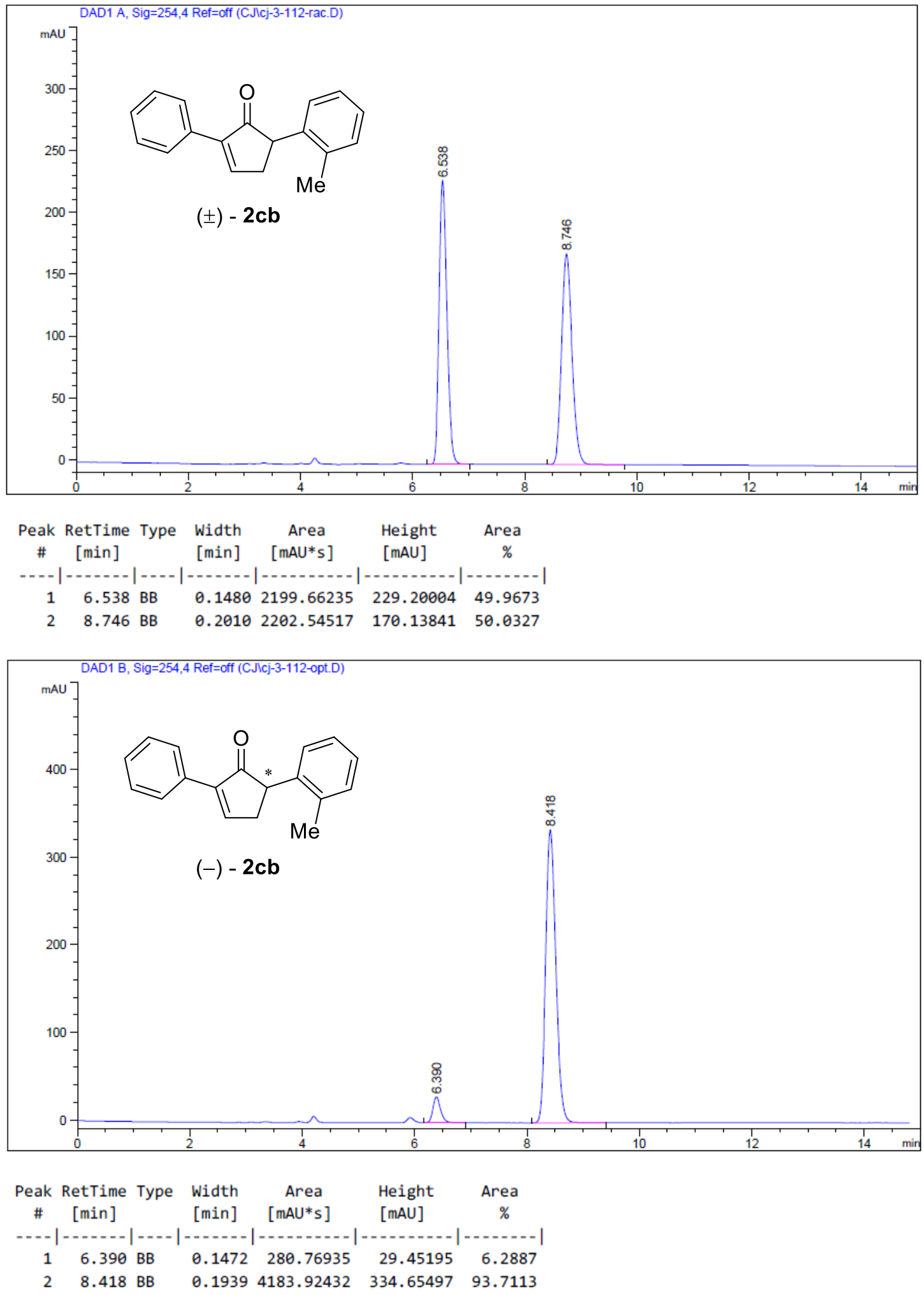
2-phenyl-5-(thiophen-3-yl)cyclopent-2-en-1-one (2cc)
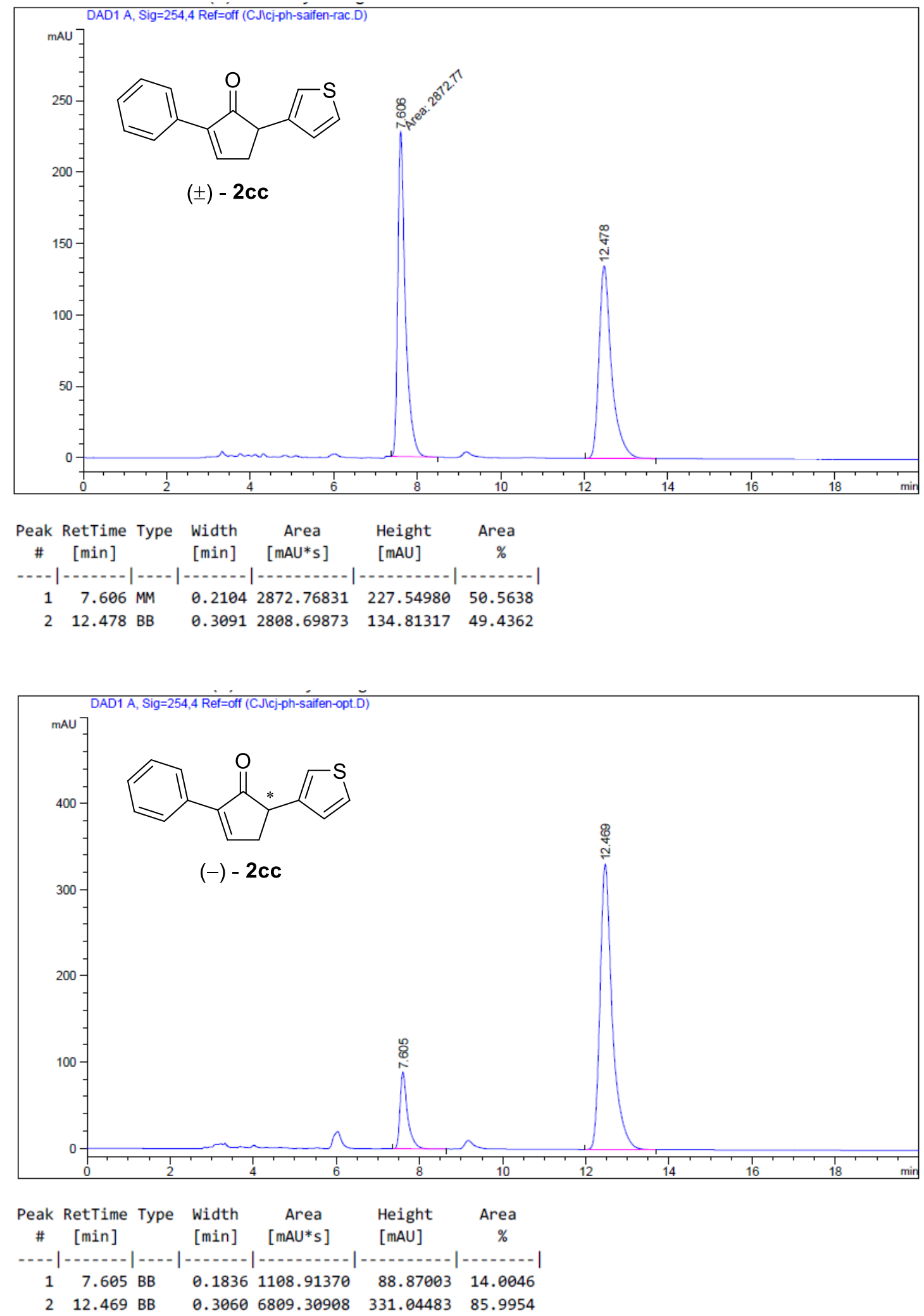
2-benzyl-5-methylcyclopent-2-en-1-one (2da)
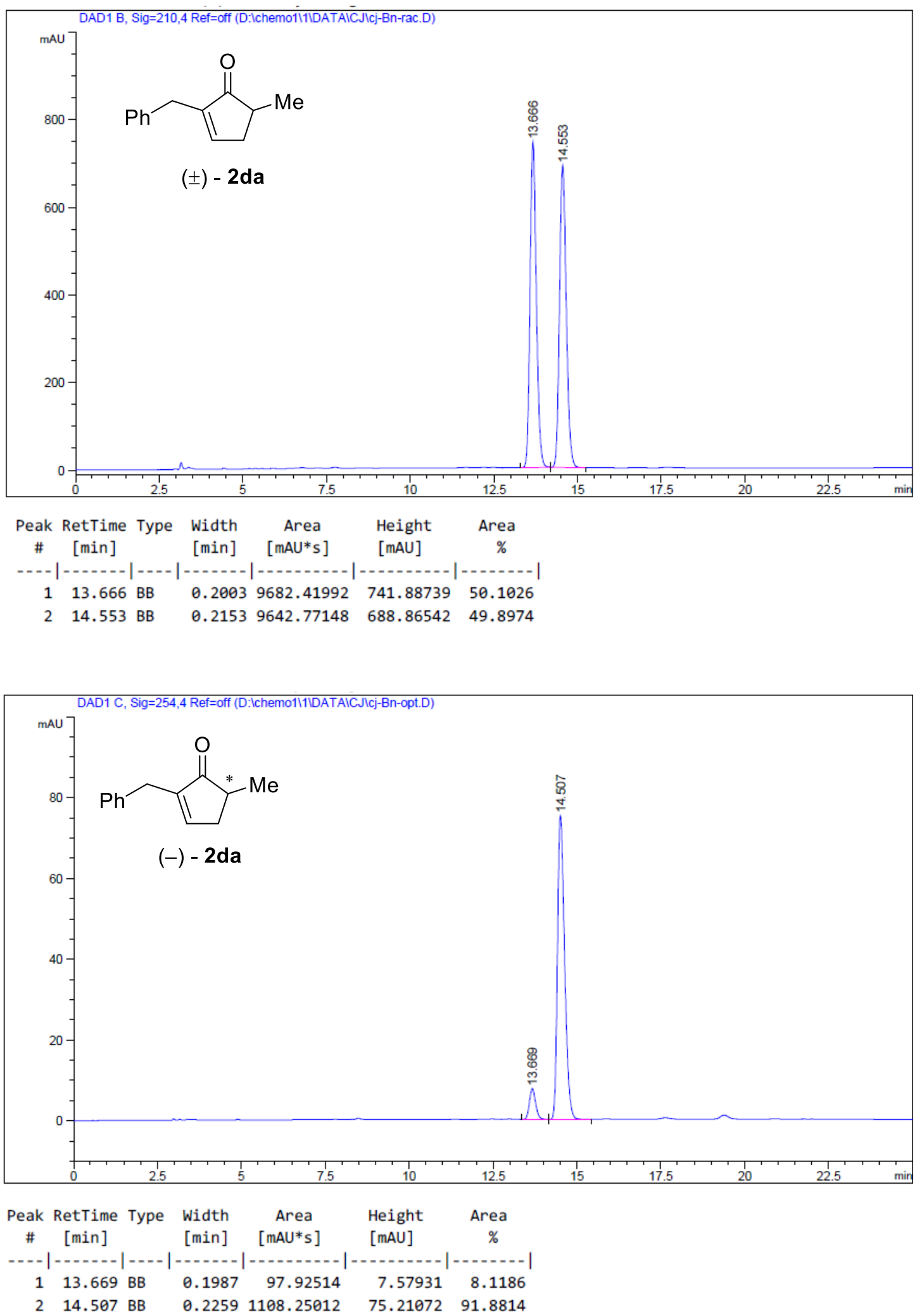
2-butyl-5-methylcyclopent-2-en-1-one (2db)
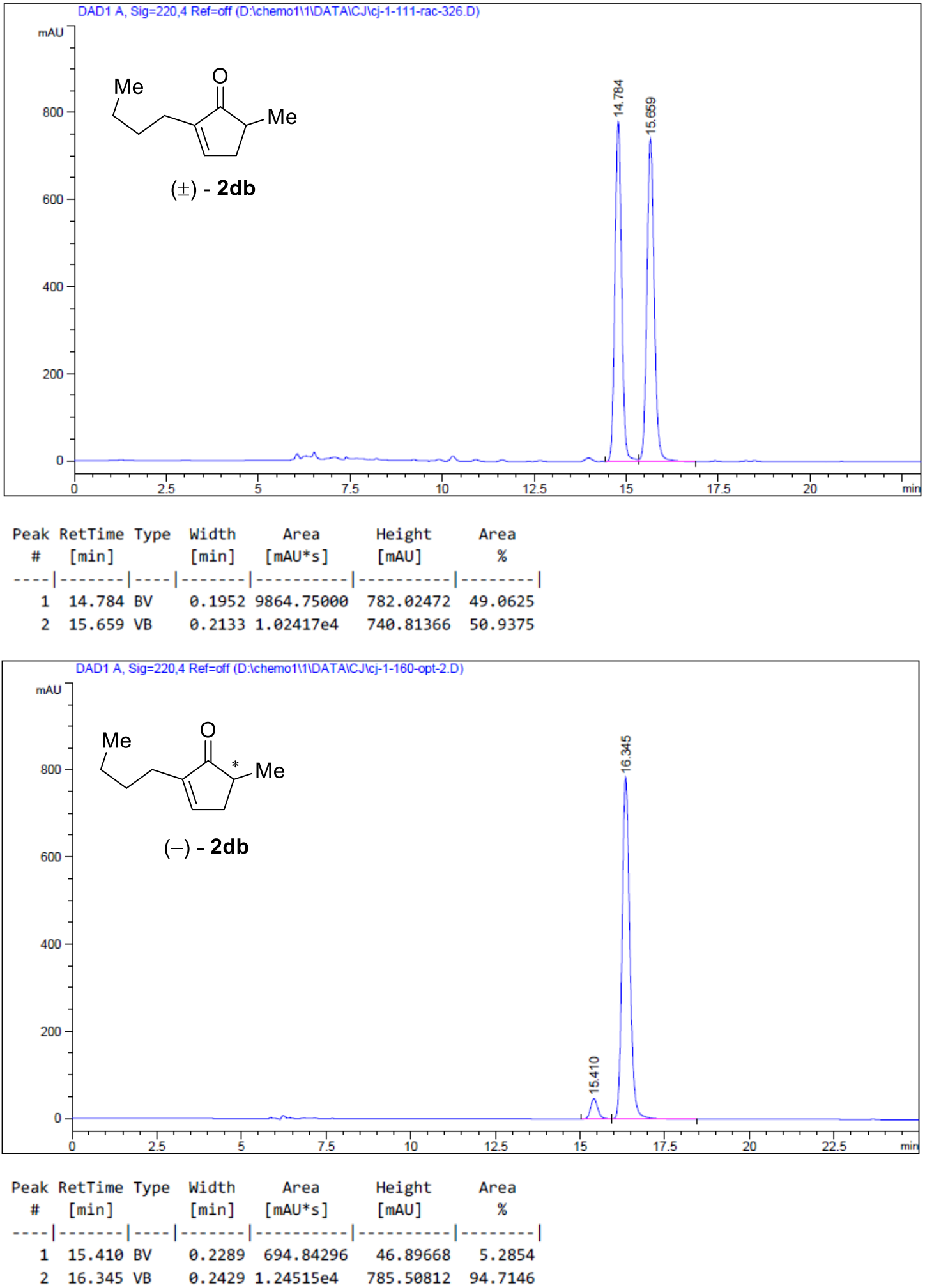
2-isopropyl-5-methylcyclopent-2-en-1-one (2dc)
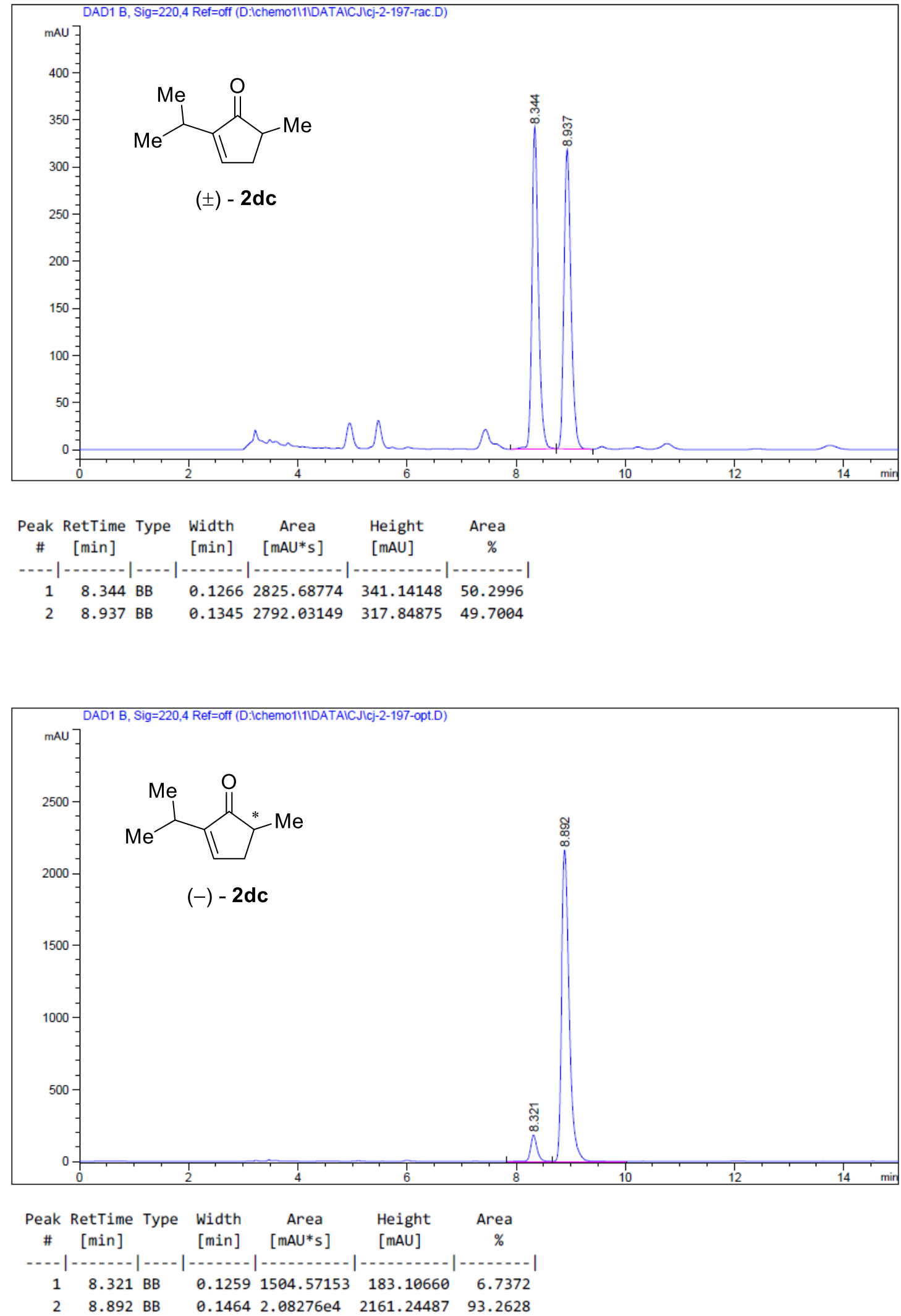
3-methyl-[1,1'-bi(cyclopentan)]-5-en-2-one (2dd)
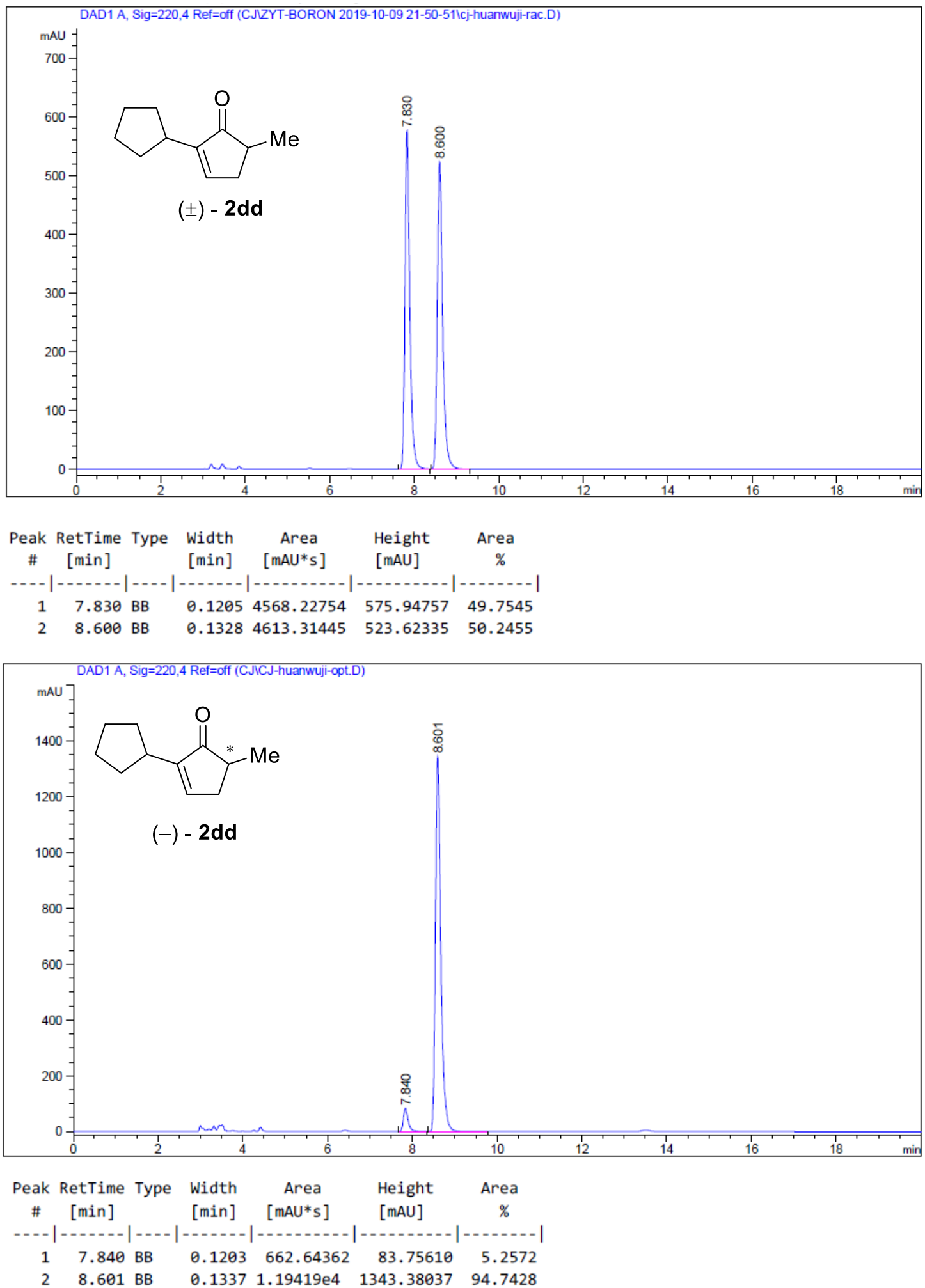
2-cyclohexyl-5-methylcyclopent-2-en-1-one (2de)
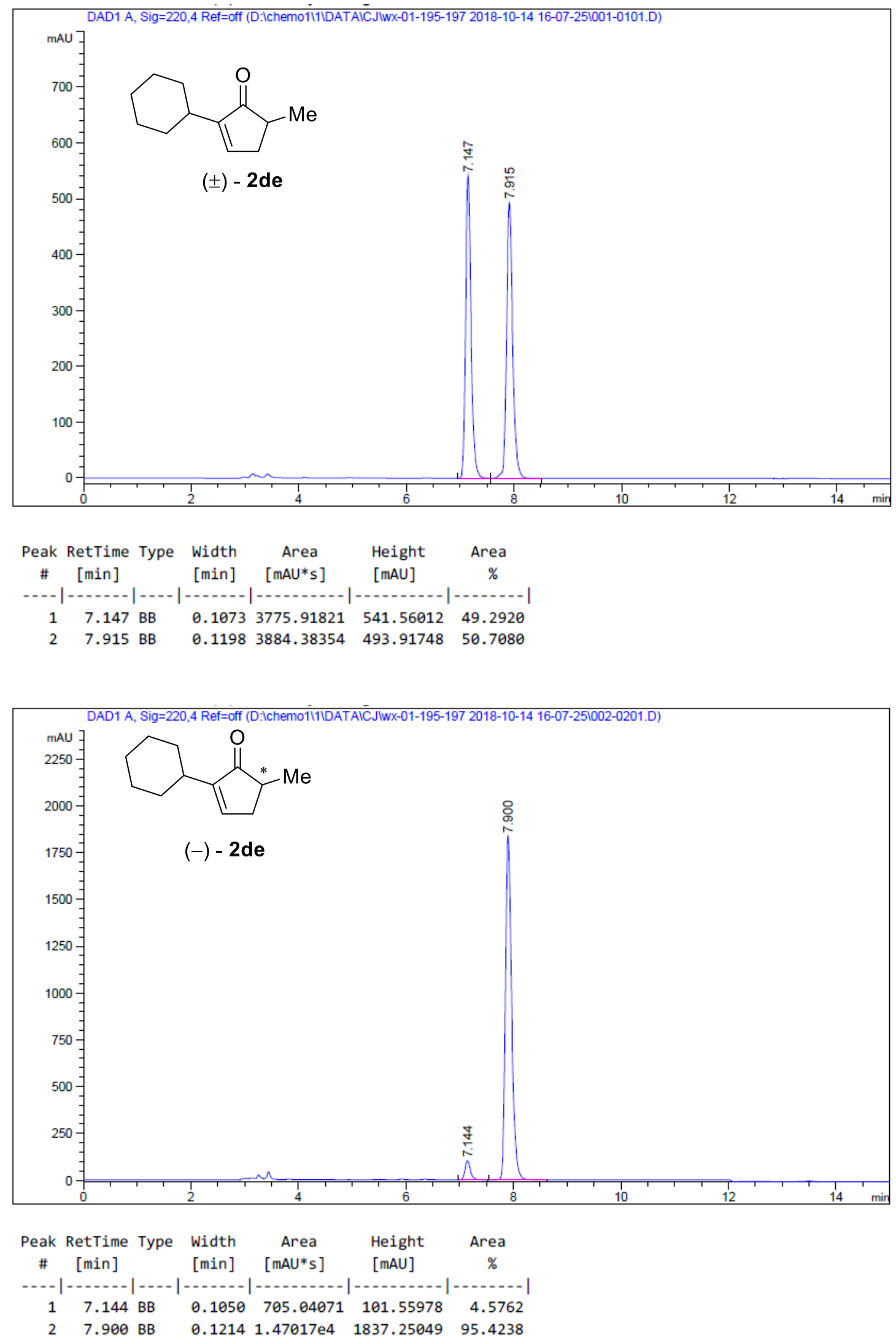
2-(cyclohex-1-en-1-yl)-5-methylcyclopent-2-en-1-one (2ea)
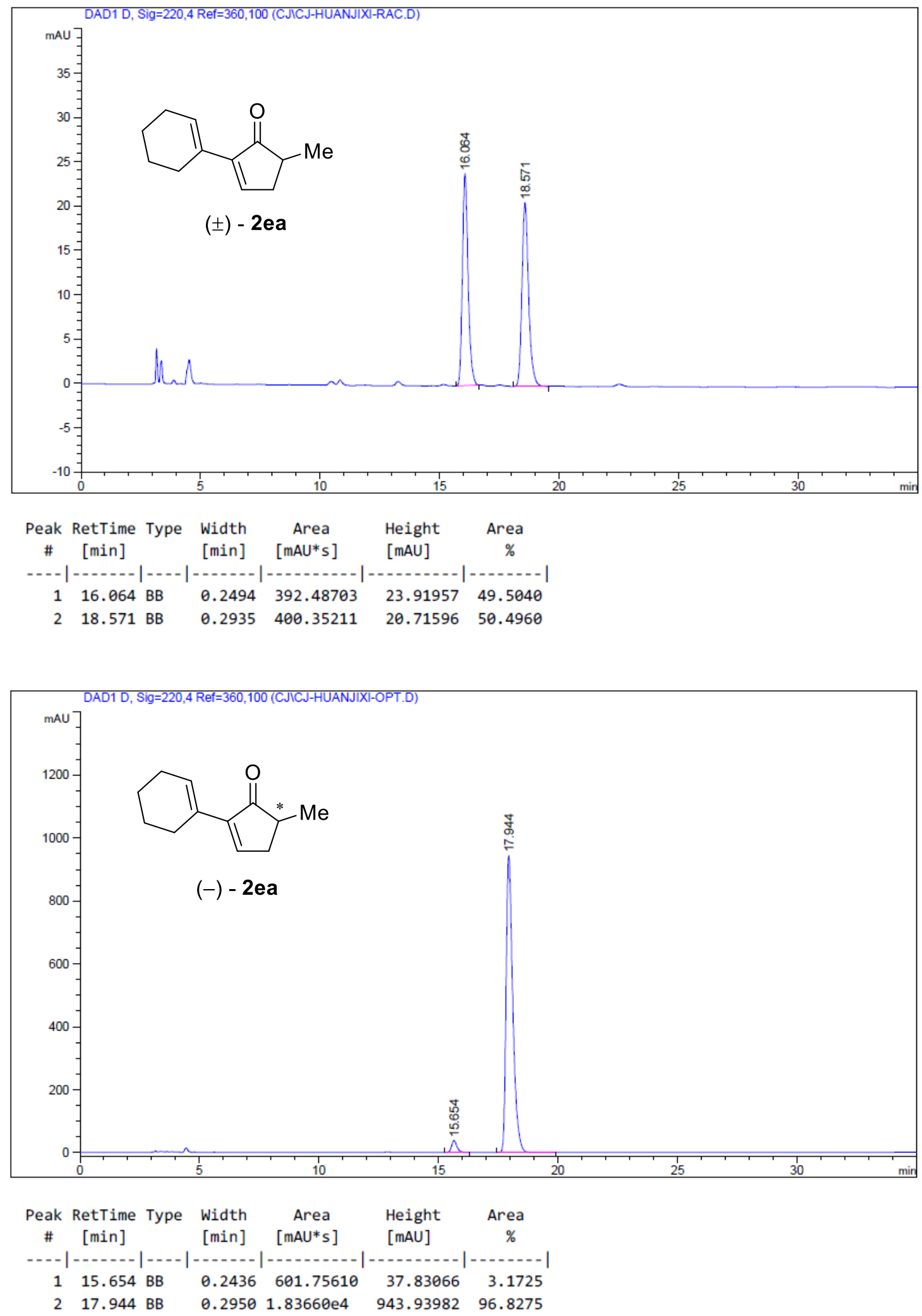
5-methyl-2-(oct-1-yn-1-yl)cyclopent-2-en-1-one (2fa)
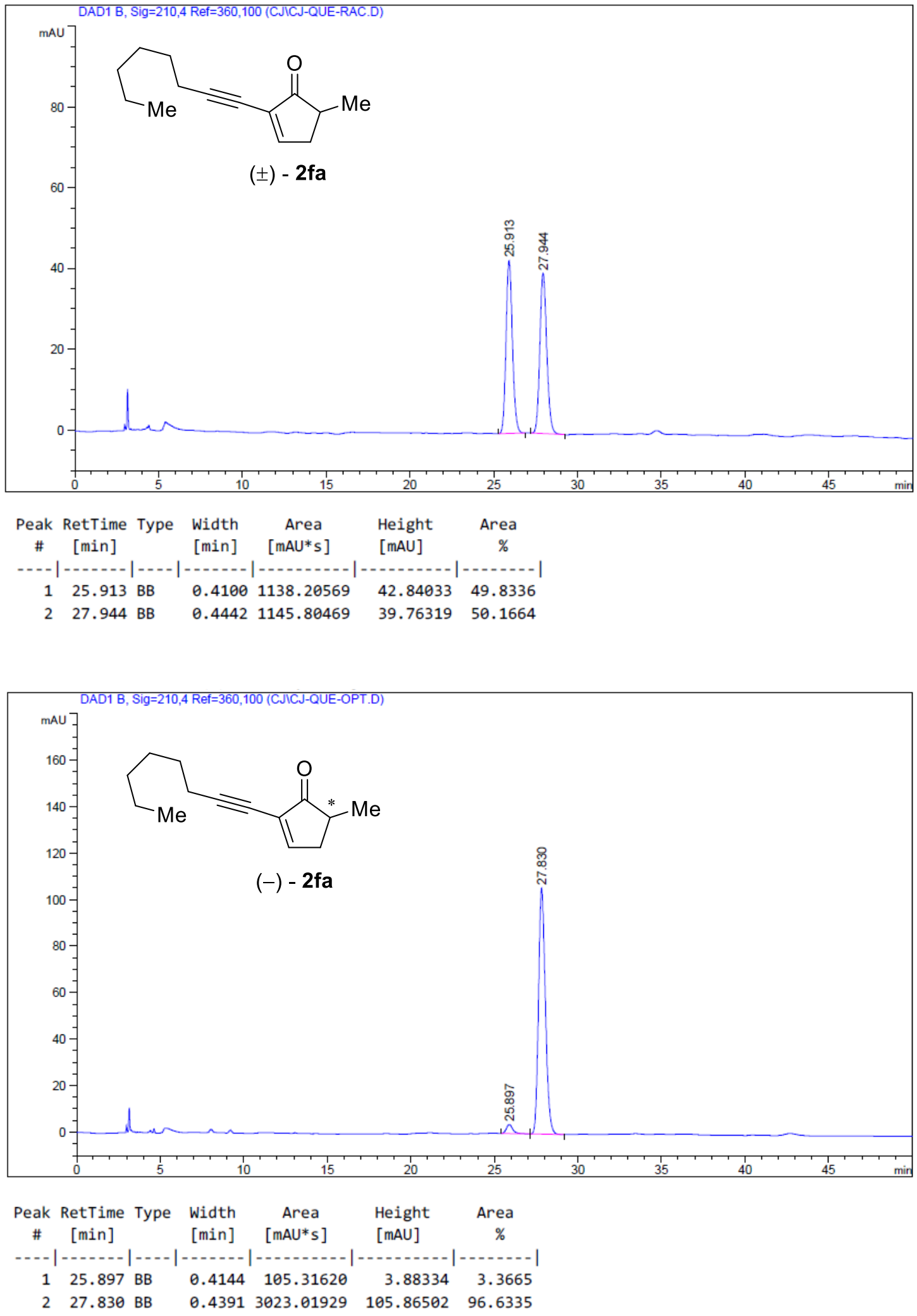
4,5-dimethyl-2-phenylcyclopent-2-en-1-one (2ga)
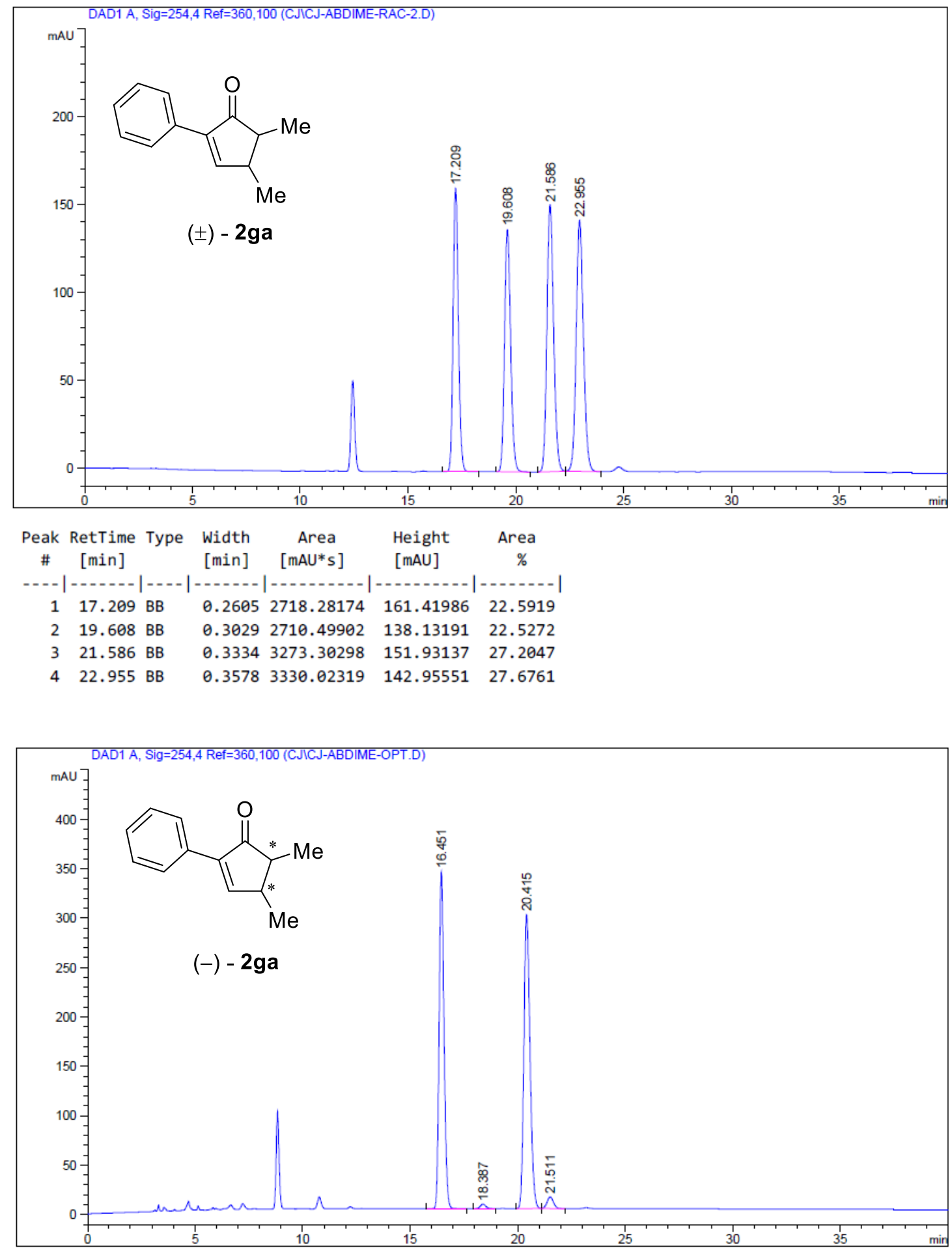

\begin{tabular}{|c|c|c|c|c|c|c|}
\hline $\begin{array}{c}\text { Peak } \\
\#\end{array}$ & $\begin{array}{c}\text { RetTime } \\
\text { [min] }\end{array}$ & Type & $\begin{array}{l}\text { Width } \\
\text { [min] }\end{array}$ & $\begin{array}{c}\text { Area } \\
{\left[\mathrm{mAU} U^{*} \mathrm{~s}\right]}\end{array}$ & $\begin{array}{l}\text { Height } \\
\text { [mAU] }\end{array}$ & $\begin{array}{c}\text { Area } \\
\%\end{array}$ \\
\hline $2+$ & |-- & & & 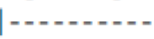 & $\cdots$ & $-\cdots$ \\
\hline 1 & 16.451 & BB & 0.2448 & 5402.15576 & 340.96735 & 46.5483 \\
\hline 2 & 18.387 & BB & 0.2715 & 81.76091 & 4.64216 & 0.7045 \\
\hline 3 & 20.415 & BB & 0.3047 & 5881.86963 & 297.46725 & 50.6818 \\
\hline 4 & 21.511 & BB & 0.3201 & 239.69850 & 11.64539 & 2.0654 \\
\hline
\end{tabular}


5-isopropyl-4-methyl-2-phenylcyclopent-2-en-1-one (2gb)

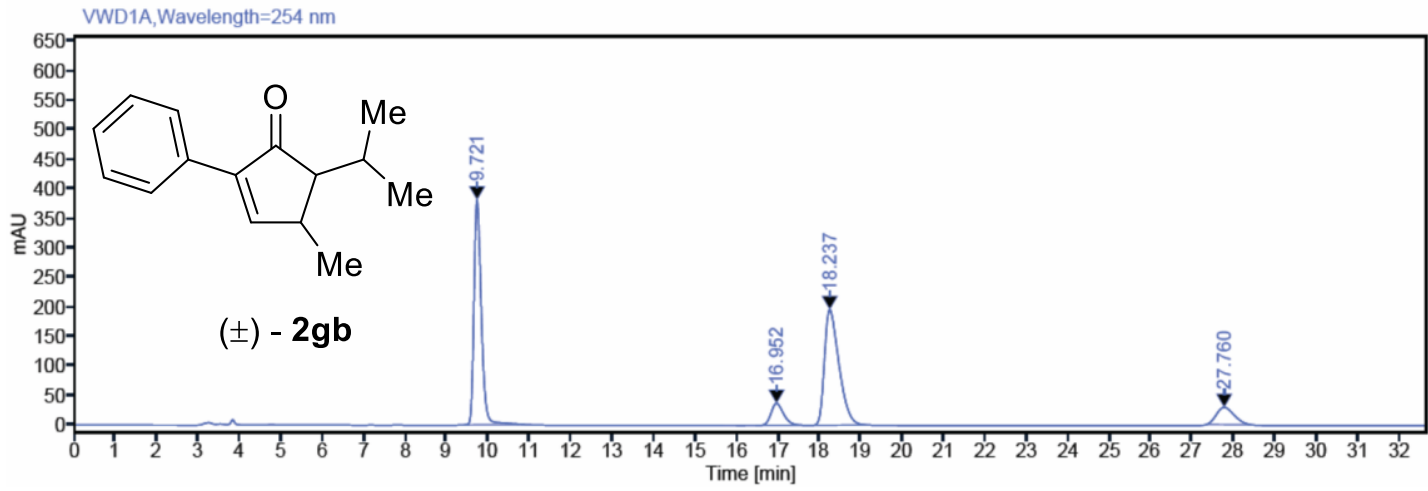

$\begin{array}{rrrrrr}\text { RT [min] } & \text { Type } & \text { Width [min] } & \text { Area } & \text { Height } & \text { Area\% } \\ 9.721 & \text { MM m } & 0.19 & 4678.65 & 381.61 & 42.17 \\ 16.952 & \text { MM m } & 0.32 & 786.83 & 37.31 & 7.09 \\ 18.237 & \text { MM m } & 0.37 & 4766.84 & 196.11 & 42.97 \\ 27.760 & \text { MM m } & 0.47 & 862.22 & 29.03 & 7.77\end{array}$

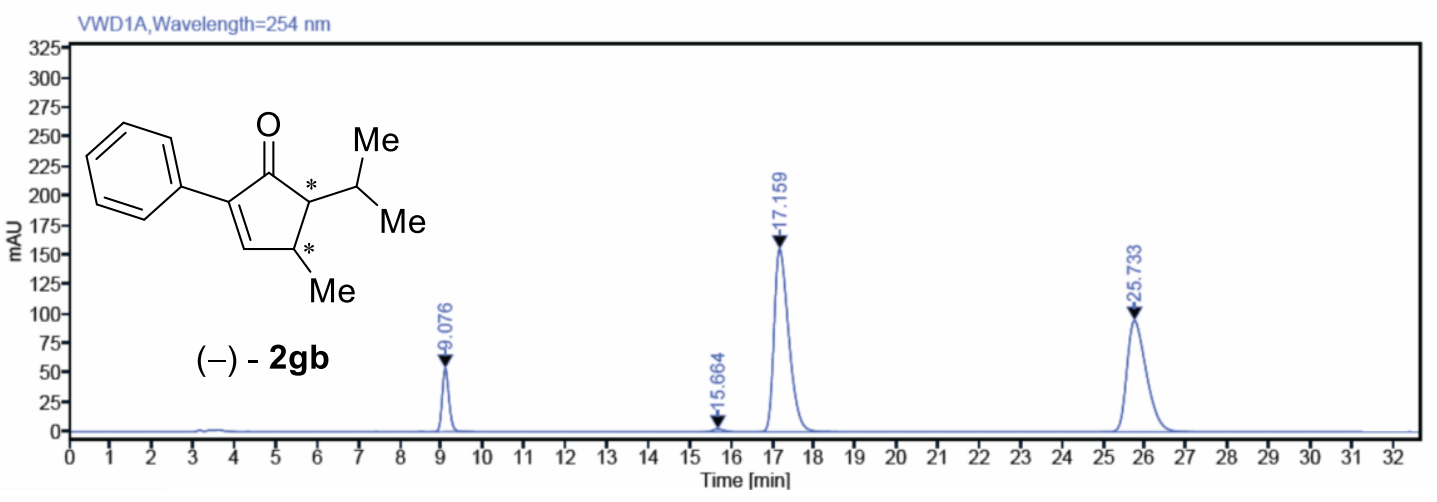

$\begin{array}{rrrrrr}\text { RT [min] } & \text { Type } & \text { Width [min] } & \text { Area } & \text { Height } & \text { Area\% } \\ 9.076 & \text { MM m } & 0.17 & 611.27 & 53.93 & 8.07 \\ 15.664 & \text { MM m } & 0.29 & 44.48 & 2.41 & 0.59 \\ 17.159 & \text { MM m } & 0.38 & 3827.50 & 155.47 & 50.53 \\ 25.733 & \text { MM m } & 0.50 & 3092.17 & 94.64 & 40.82\end{array}$




\section{5-methyl-2,4-diphenylcyclopent-2-en-1-one (2gc)}

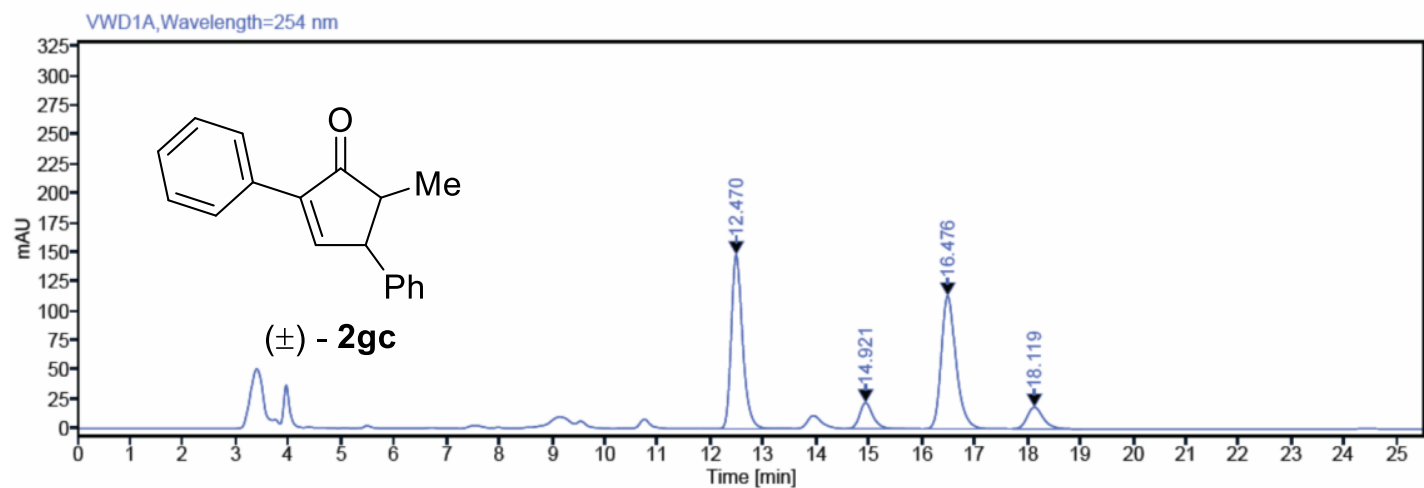

$\begin{array}{rrrrrr}\text { RT [min] } & \text { Type } & \text { Width [min] } & \text { Area } & \text { Height } & \text { Area\% } \\ 12.470 & \text { BV } & 1.02 & 2203.93 & 148.21 & 42.49 \\ 14.921 & \text { BB } & 1.11 & 385.41 & 22.06 & 7.43 \\ 16.476 & \text { BB } & 1.65 & 2210.77 & 113.19 & 42.62 \\ 18.119 & \text { BB } & 1.33 & 386.96 & 18.43 & 7.46\end{array}$

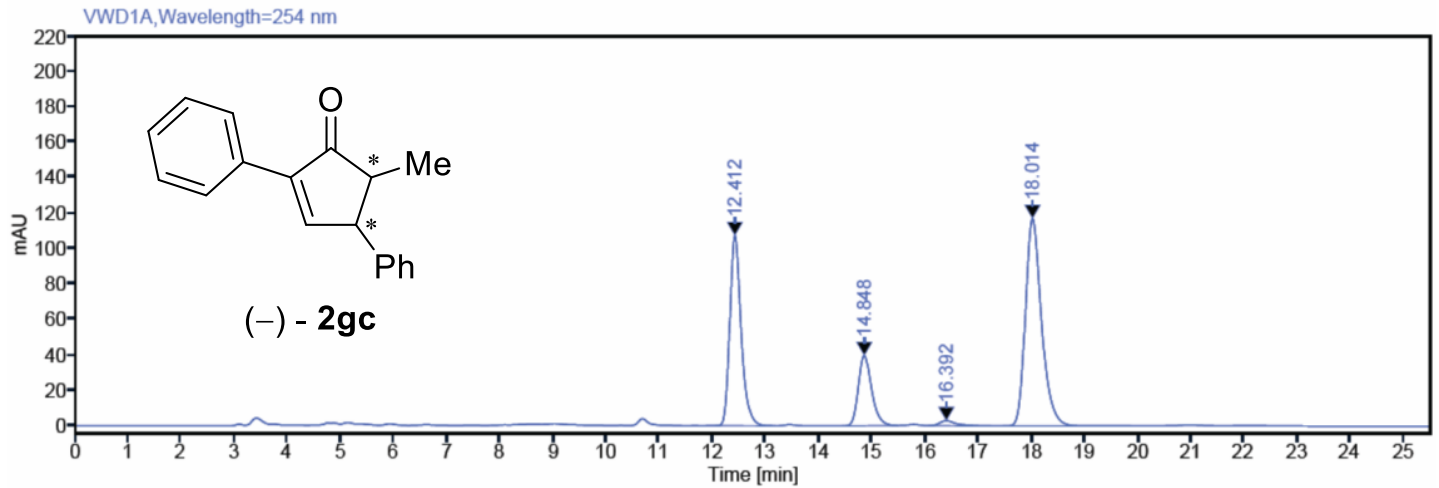

$\begin{array}{rrrrrr}\text { RT [min] } & \text { Type } & \text { Width [min] } & \text { Area } & \text { Height } & \text { Area\% } \\ 12.412 & \text { BB } & 1.13 & 1588.18 & 107.30 & 32.99 \\ 14.848 & \text { BB } & 1.05 & 690.67 & 39.59 & 14.35 \\ 16.392 & \text { BB } & 1.17 & 60.43 & 2.86 & 1.26 \\ 18.014 & \text { BB } & 2.41 & 2474.77 & 117.06 & 51.41\end{array}$


5-methyl-2,3-diphenylcyclopent-2-en-1-one (2ha)
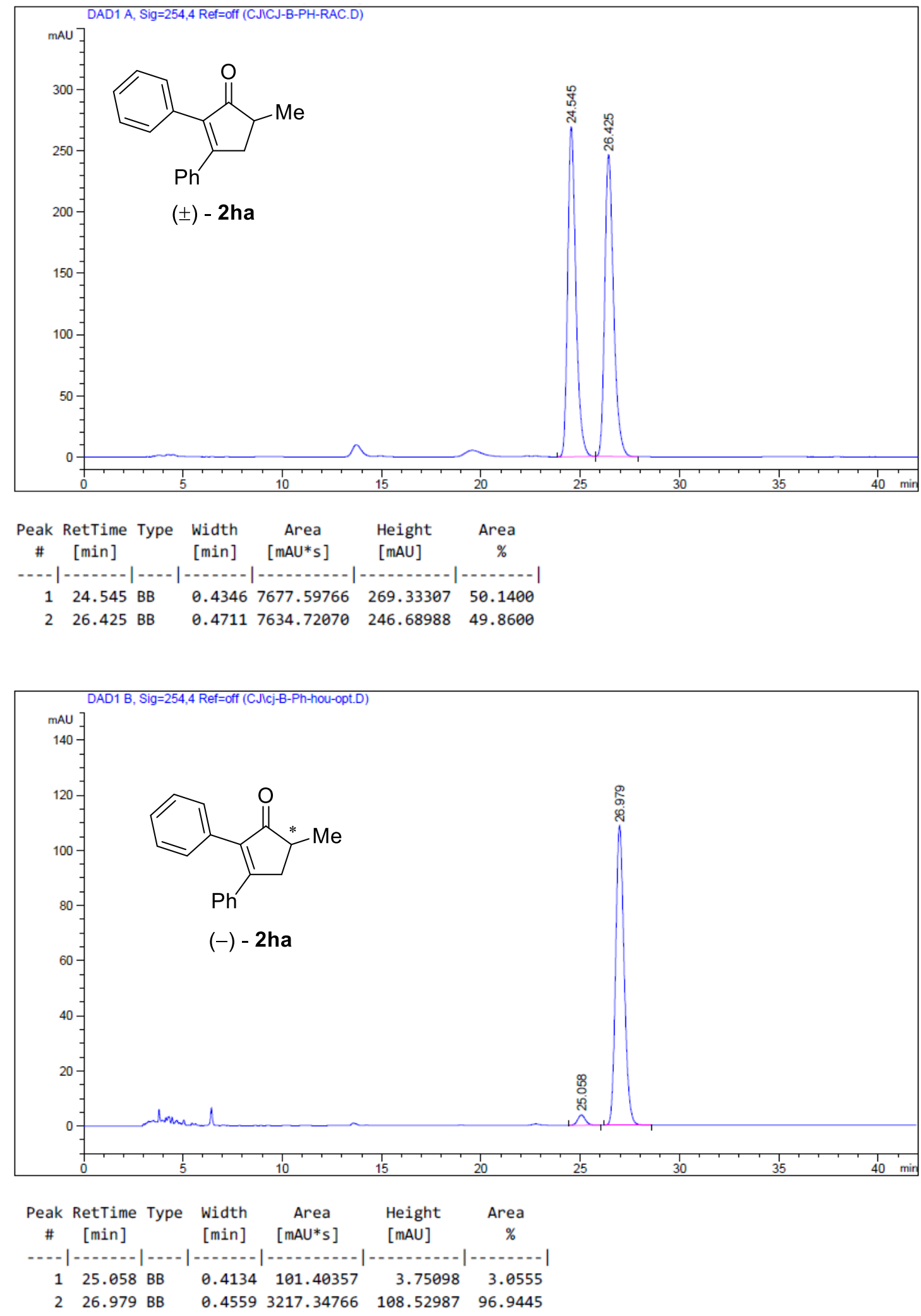


\section{4,4,5-trimethyl-2-phenylcyclopent-2-en-1-one (2ia)}
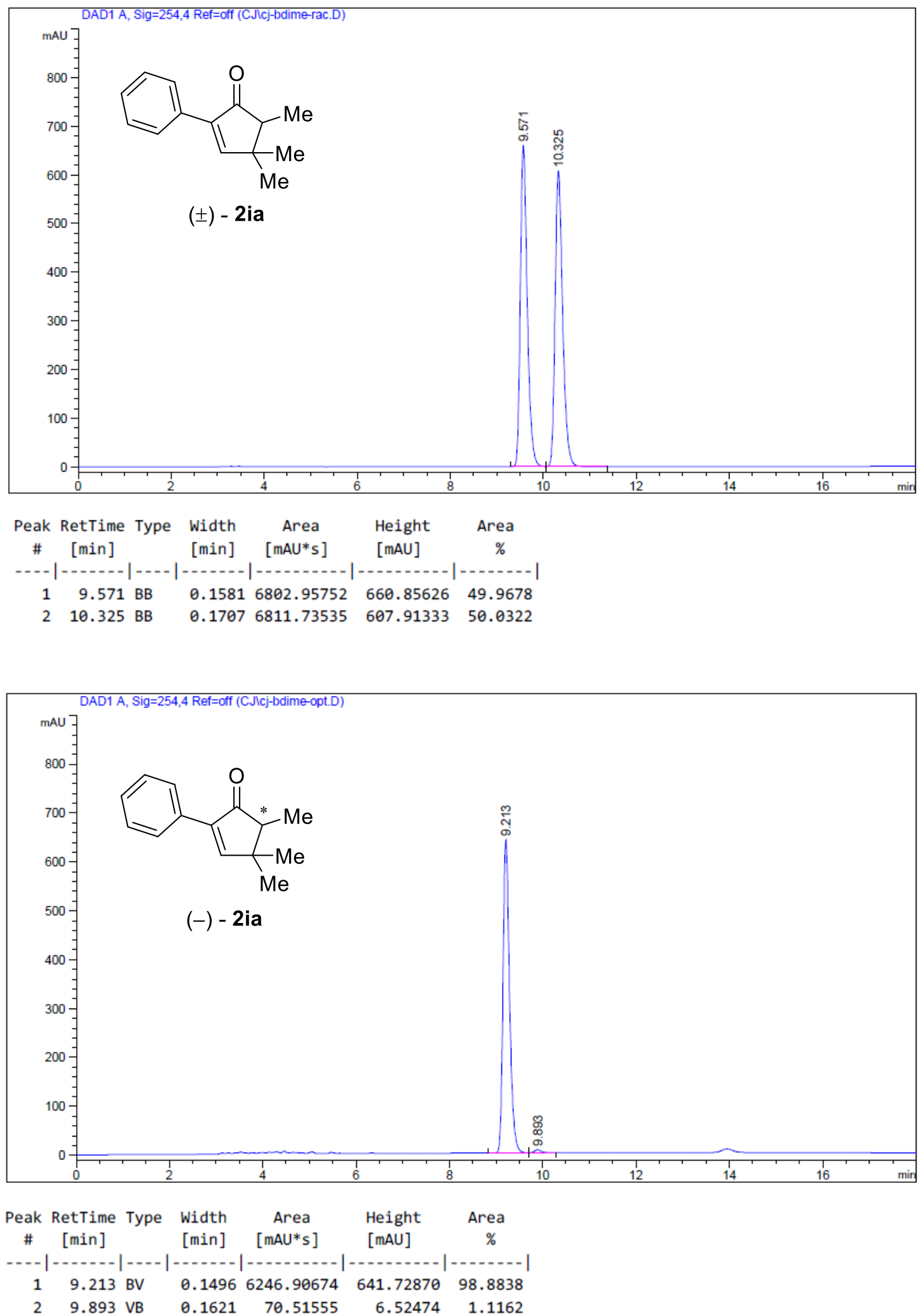
5-methyl-2-phenylcyclopent-2-en-1-one (2aa (from 1ja))
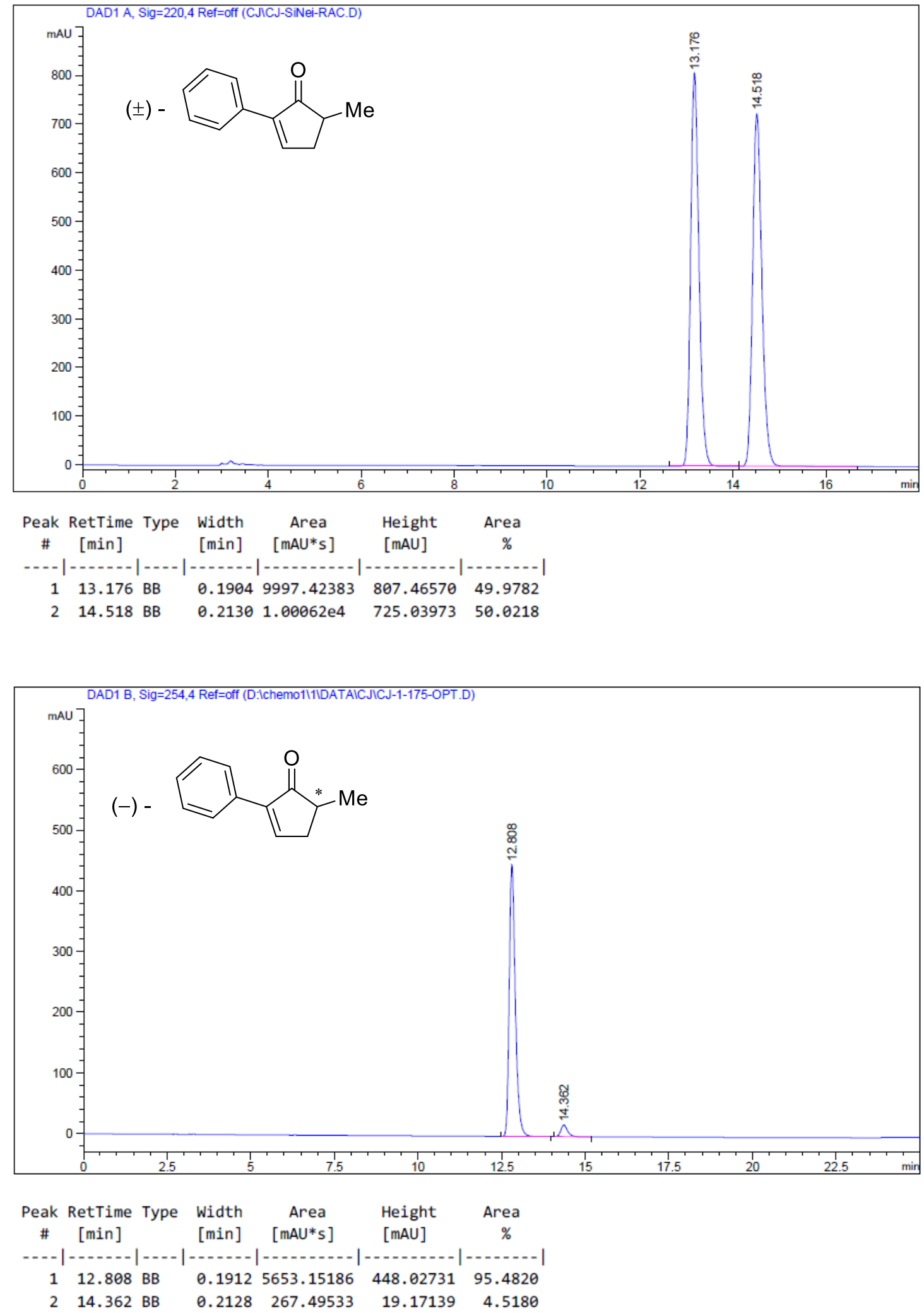


\section{methyl}

2-((1R,4R)-3-((tert-butyldimethylsilyl)oxy)-4-methyl-2-phenylcyclopent-2-en-1-yl )acetate (5)
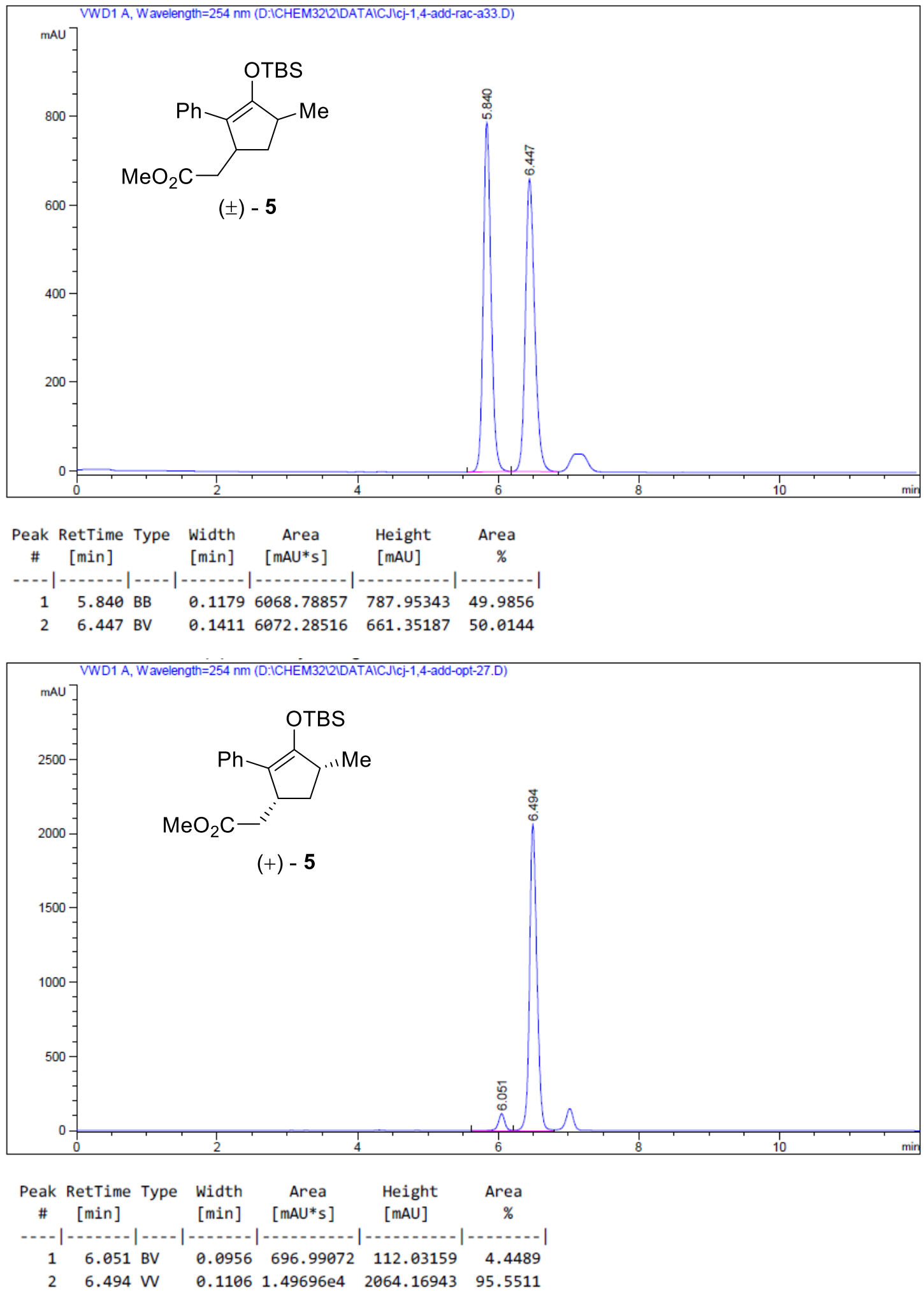
6-methyl-3-phenyl-5,6-dihydropyridin-2(1H)-one (6)
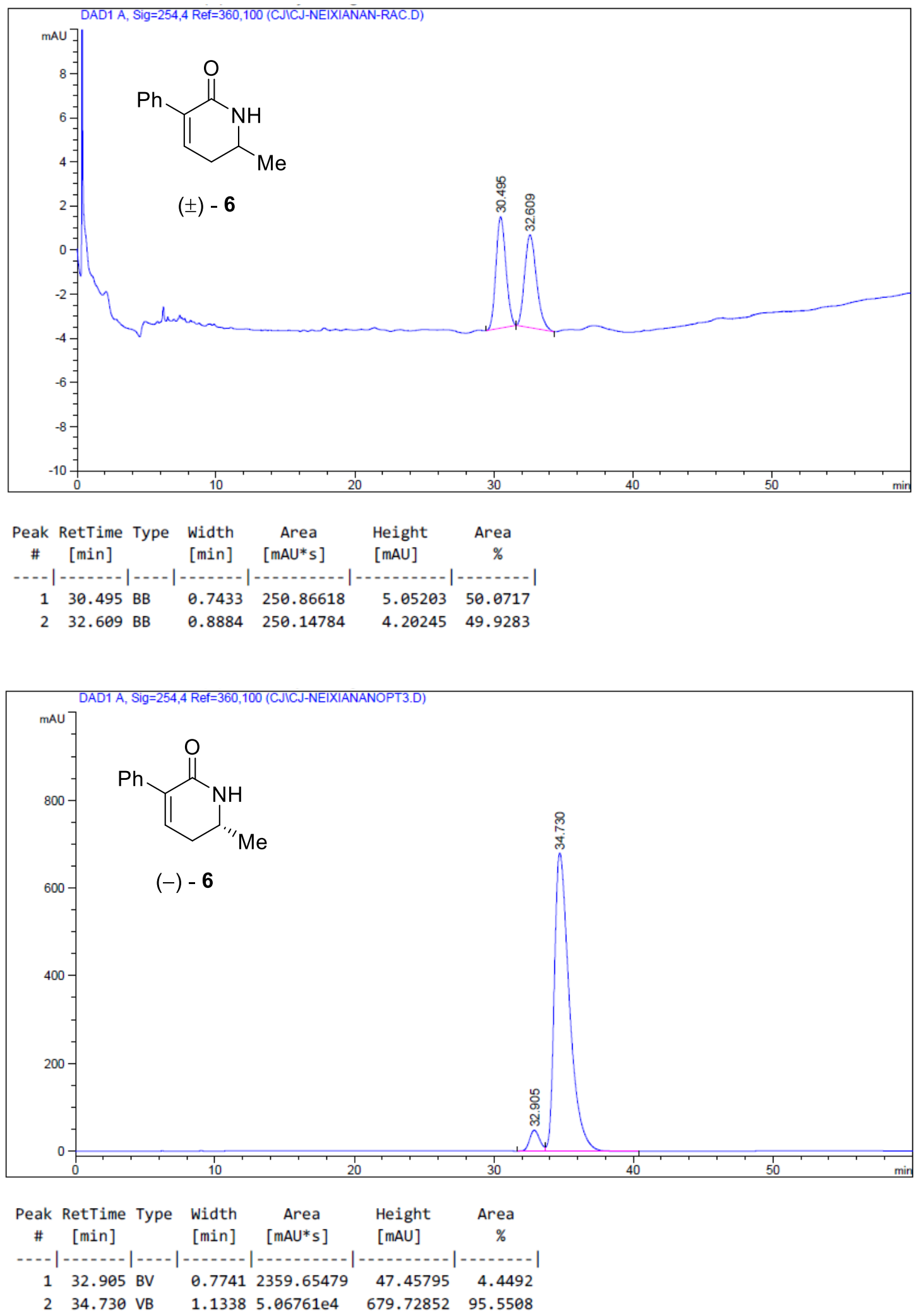
5-methyl-2-phenylcyclopent-2-en-1-ol (7)
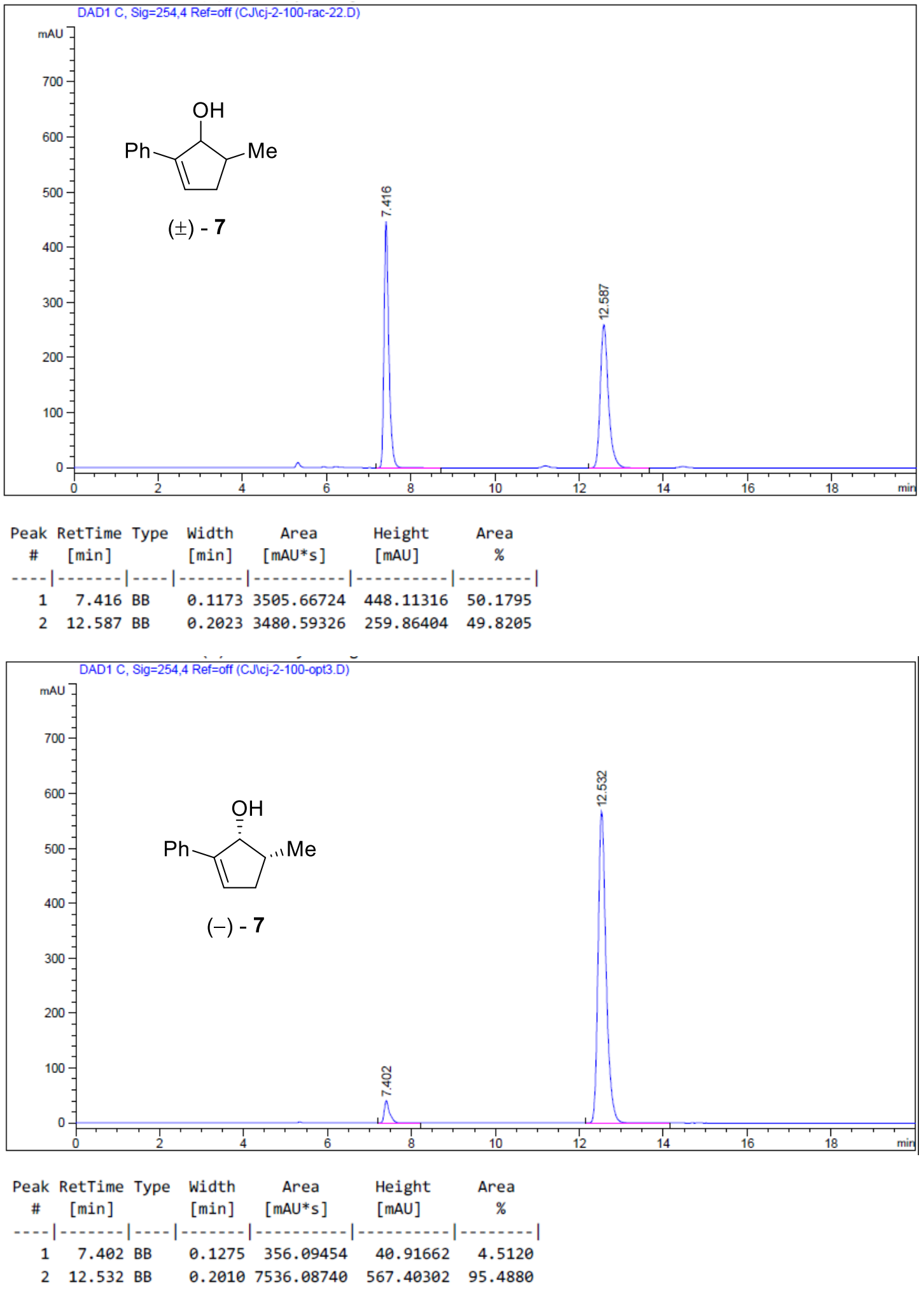
N,N-dimethyl-2-(4-methyl-2-phenylcyclopent-2-en-1-yl)acetamide (8)
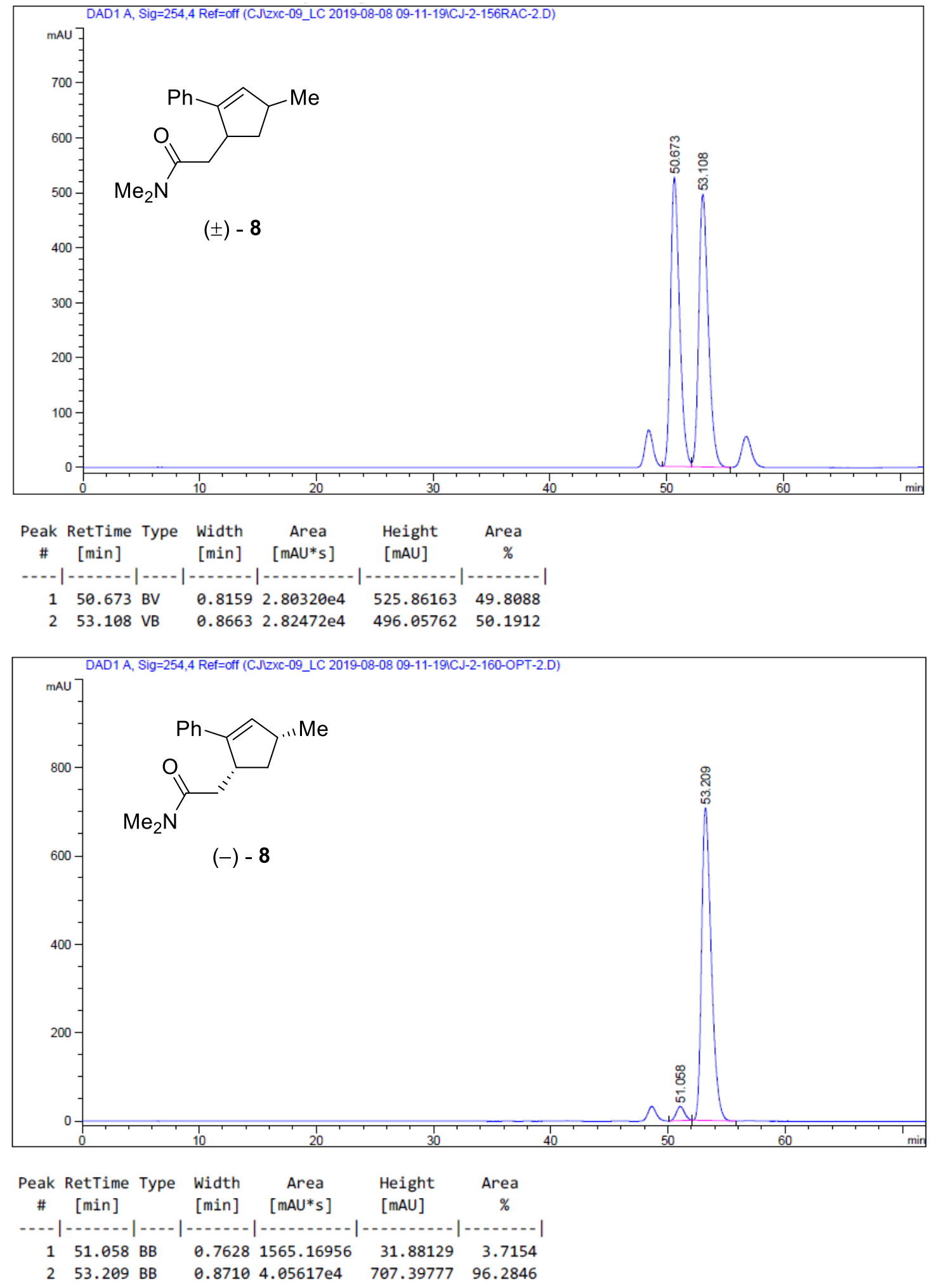


\section{References}

1. Armarego, W. L. F.; Chai, C. L. L. Purification of Laboratory Chemicals-Six Edition; Elsevier Inc.: London, 2009.

2. Cai, M. Z.; Zhou, Z.; Jiang, J. W. A facile stereoselective synthesis of (2E)-3-silylallylic alcohols by hydromagnesiation of 1-aryl-2-silylacetylenes. Eur. J. Org. Chem. 2006, 1400-1402.

3. Wang, G.-P.; Chen, M.-Q.; Zhu, S.-F.; Zhou, Q.-L. Enantioselective Nazarov cyclization of indole enones cooperatively catalyzed by Lewis acids and chiral Brønsted acids. Chem. Sci. 2017, 8, 7197-7202.

4. Erkkilä, A.; Pihko, P. M. Mild organocatalytic $\alpha$-methylenation of aldehydes. $J$. Org. Chem. 2006, 71, 2538-2541.

5. Jacobi, P. A.; Cann, R. O.; Skibbie, D. F. A convenient synthesis of methylenomycin B. Further mechanistic studies on the SET catalyzed electrocyclization of enynones. Tetrahedron Lett. 1992, 33, 2265-2268.

6. Hon, Y. S.; Chang, F. J.; Lu, L.; Lin, W. C. Preparation of $\alpha$-substituted acroleins via the reaction of aldehyde or the corresponding ozonide with dihalomethane and diethylamine. Tetrahedron 1998, 54, 5233-5246.

7. Walkowiaka, J.; Campoa, T. M.; Amedurib, B.; Gouverneur, V. Syntheses of mono-, di-, and trifluorinated styrenic monomers. Synthesis 2010, 11, 1883-1890.

8. Mitchell, T. N.; Wickenkamp, R.; Amamria, A.; Dicke, R.; Schneider, U. Formation and reactions of olefins with vicinal silyl and stannyl substituents. $J$. Org. Chem. 1987, 52, 4868-4874.

9. Jung, M. E.; Ho, D.; Chu, H. V. Synthesis of highly substituted cyclohexenes via mixed Lewis acid-catalyzed Diels-Alder reactions of highly substituted dienes and dienophiles. Org. Lett. 2005, 7, 1649-1651.

10. Marsili, L. A.; Pergomet, J. L.; Gandon, V.; Riveira, M. J. Iodine-Catalyzed Iso-Nazarov Cyclization of Conjugated Dienals for the Synthesis of 2-Cyclopentenones. Org. Lett. 2018, 20, 7298-7303. 